\title{
Experimental investigation of shock wave - bubble interaction
}

\author{
Dissertation \\ zur Erlangung des Doktorgrades \\ der Mathematisch-Naturwissenschaftlichen Fakultäten \\ der Georg-August-Universität zu Göttingen
}

vorgelegt von

Mohsen Alizadeh

aus Kerman, Iran

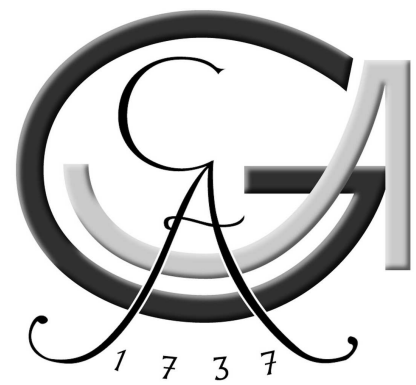

Göttingen 2010 
D7

Referent: Prof. Dr. Werner Lauterborn

Korreferent: Prof. Dr. Ulrich Parlitz

Tag der mündlichen Prüfung: 09.04.2010 


\section{Contents}

$\begin{array}{llr}1 & \text { Introduction } & \mathbf{8}\end{array}$

2 Outline of previous investigations $\quad \mathbf{1 1}$

3 Theory 24

3.1 Shock wave propagation . . . . . . . . . . . . . 24

3.1.1 Simulation of pressure wave propagation . . . . . . . . 29

3.1.2 Reflection of shock waves . . . . . . . . . . . 32

3.2 Spherical bubble dynamics . . . . . . . . . . . . 35

3.3 Shock wave - bubble interaction . . . . . . . . . . . . . . 39

3.3.1 Jet velocity and impact pressure . . . . . . . . . . . . 42

3.3.2 Kelvin impulse and kinetic energy . . . . . . . . . . . 42

4 Shock wave characterization $\quad 44$

4.1 Generation of lithotripter shock waves . . . . . . . . . . . . . . 44

4.1 .1 Experimental method . . . . . . . . . . . . . 45

4.2 Focusing of the shock waves in a cuvette . . . . . . . . . . . . 48

4.3 Measurement of the lithotripter shock wave (LSW) profile . . . 49

4.4 Calibration ...................... 50

4.5 Deconvolution ....................... 51

4.6 Shock wave pressure profile . . . . . . . . . . . 52

4.7 Bubble formation . . . . . . . . . . . . . . . . . . 55

4.8 Shock wave propagation in water . . . . . . . . . 57

$\begin{array}{lll}5 & \text { Shock wave }- \text { bubble interaction } & \mathbf{6 1}\end{array}$

5.1 Method ....................... 61

5.2 Laser generation of cavitation bubbles . . . . . . . . . . . 64

5.3 Secondary cavitation . . . . . . . . . . . . 66

5.4 Pressure field measurements . . . . . . . . . . . . . . 67

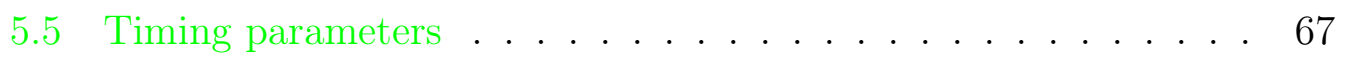

5.6 Experimental results: Part I . . . . . . . . . . . . . . . . 69

5.6.1 Pressure enhancement . . . . . . . . . . . 72

5.6 .2 Reduction of collapse time . . . . . . . . . . . . . 73

5.6.3 Lithotripter shock wave (LSW) pulse amplitude . . . . . 74

5.6.4 Bubble dynamics under the influence of the applied lithotripter shock wave $(\mathrm{LSW}) \quad \ldots \ldots \ldots$. . . . . . . 76

5.7 Experimental results: Part II . . . . . . . . . . . . . . 82

5.7 .1 Pressure enhancement . . . . . . . . . . 85 
5.7 .2 Reduction of collapse time . . . . . . . . . . 86

5.7.3 Lithotripter shock wave (LSW) pulse amplitude . . . . . 87

5.7.4 Bubble dynamics under the influence of the applied lithotripter

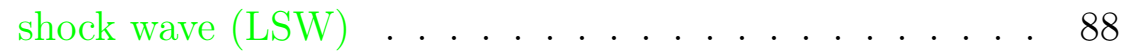

5.8 Experimental results: Part III . . . . . . . . . . . . . . . . 93

5.8.1 Pressure enhancement . . . . . . . . . . . . . 95

5.8.2 Reduction of collapse time . . . . . . . . . . . 96

5.8.3 Lithotripter shock wave (LSW) pulse amplitude . . . . . 97

5.9 Conclusions . . . . . . . . . . . . . . . . . 97

6 Shock wave - bubble interaction in the vicinity of a rigid $\begin{array}{lr}\text { boundary } & 99\end{array}$

6.1 Method ....................... . . 99

6.2 Experimental results: part I . . . . . . . . . . . . . 100

6.3 Experimental results: part II . . . . . . . . . . . . . . . . . . 101

6.3.1 Pressure enhancement . . . . . . . . . . . 105

6.3.2 Reduction of collapse time . . . . . . . . . . . 106

6.3.3 Bubble migration . . . . . . . . . . . . . . . 107

6.4 Experimental results: part III . . . . . . . . . . . . . . . 111

6.4.1 Pressure amplification and reduction of collapse time . . 114

6.5 Conclusions ..................... . . 115

$\begin{array}{lr}\text { Bibliography } & 116\end{array}$

$\begin{array}{lr}\text { Acknowledgment } & \mathbf{1 2 5}\end{array}$ 


\section{Summary}

The behavior of bubbles exposed to an incident shock wave in a liquid is one of the main issues concerning bubble dynamics. This phenomenon plays an important role in several practical applications such as ultrasound cleaning, drug delivery, gene transfer and shock wave lithotripsy.

In this work, the dynamics of laser-generated single cavitation bubbles exposed to lithotripter shock waves has been investigated experimentally. The energy of the impinging shock wave is varied in several steps. High-speed photography and pressure field measurements simultaneously with image acquisition provide the possibility of capturing the fast bubble dynamics under the effect of the shock wave impact. The pressure measurement is performed using a fiber optic probe hydrophone (FOPH) which operates based on optical diagnostics of the shock wave propagating medium.

After a short introduction in chapter 1 an overview of the previous studies in chapter 2 is presented. The reported literatures include theoretical and experimental investigations of several configurations of physical problems in the field of bubble dynamics. In chapter 3 a theoretical description of propagation of a shock wave in a liquid like water has been discussed. Different kinds of reflection of a shock wave at an interface are taken into account. Undisturbed bubble dynamics as well as interaction between a planar shock wave and an initially spherical bubble are explored theoretically. Some physical parameters which are important in this issue such as the velocity of the shock-induced liquid jet, Kelvin impulse and kinetic energy are explained.

The shock waves are generated in a water filled container by a focusing piezoelectric generator. The shock wave profile has a positive part with pulse duration of $\approx 1 \mu \mathrm{s}$ followed by a longer tension tail (i.e. $\approx 3 \mu \mathrm{s}$ ). In chapter 4 high-speed images depict the propagation of a shock wave in the water filled tank. The maximum pressure is also derived for different intensity levels of the shock wave generator. The measurement is performed in the free field (i.e. in the absence of laser-generated single bubbles).

In chapter 5 the interaction between lithotripter shock waves and laserinduced single cavitation bubbles is investigated experimentally. An expanded beam of a Q-switched laser pulse at wavelength of $\lambda=532 \mathrm{~nm}$ and with pulse duration of $\approx 4 \mathrm{~ns}$ is focused at the center of a water tank using an aberra- 
tion minimized lens design. Single cavitation bubbles are initiated via optical breakdown [52] at this location which coincides with the position of which the shock wave is focused. The energy of the shock wave source has been altered in 8 steps. The pressure pulse amplitude of the impinging shock wave measured at the distance of about $1.8 \mathrm{~mm}$ above the focus location range from $24.4 \mathrm{MPa}$ to 108.1 MPa. The lithotripter shock wave impact time is varied in three steps which provides the possibility of investigation of the bubble dynamics in both cases of collapsing and expanding cavities at the moment of the shock wave impingement. After the shock wave impact, the bubble spherical symmetry is broken and a liquid jet develops in the original direction of the shock propagation. The speed of the jet is increasing with the shock wave energy.

Due to the energy transfer from the shock wave to the bubble, the forced cavity implosion is more violent in comparison to free oscillation. The pressure pulse amplitude released from the forced bubble collapse is amplified and the collapse time is reduced. These effects are discussed in chapter 5. Generally, when the bubble is collapsing at the time of the shock impact, the forced cavity collapse is more violent with a resultant of more pressure enhancement compared to the expanding bubbles at the moment of the shock arrival. The maximum pressure enhancement and reduction of bubble collapse time occur when the time interval between the moments of the shock impact and bubble collapse approaches the pulse duration of the compression part of the shock wave profile (i.e. $\approx 1 \mu \mathrm{s}$ ). For each specific shock wave arrival time, increasing the shock intensity leads to the fact that the bubble collapse takes place earlier relative to the moment of the shock impact and having more collapse pressure amplification. The pressure can be enhanced by a factor of up to about 4 compared with the free bubble collapse pressure.

The bubble centroid is translated in the original direction of the shock wave passage because of the momentum transfer from the shock to the bubble. This translation is more pronounced in the case that the cavity is collapsing at the moment of the shock wave arrival.

The pressure profile measured above the laser-induced bubbles is broadened due to diffraction and absorption effects. Thus, the shock wave pulse amplitude measured at the top of the single bubbles is less than the one obtained in the free field. The amount of damping in the maximum shock pressure is increasing with the shock wave intensity.

The dynamics of laser-induced single cavitation bubbles near to a solid wall under the influence of a lithotripter shock wave is investigated in chapter 6 . The boundary is located parallel to the direction of the shock wave propagation. The distance between interface and the cavity is altered in several steps as well as the moment of the shock impact. The presence of the wall leads to an increase of the bubble collapse time while the shock wave tends to decrease it. It is shown that by increasing the distance between boundary and the bubble, the effect of the shock wave becomes more prominent. The bubble tip is inclined toward the wall and at large cavity distances, this inclination lowers and the liquid jet is developed in the direction of the shock wave passage. 
For a specific distance between cavity and the boundary, it is shown that the implosion of initially collapsing cavities is more violent compared to initially expanding bubbles. 


\section{Chapter 1}

\section{Introduction}

Clouds of bubbles can be formed in a liquid when the negative pressure is greater than a threshold value. This phenomenon is called "Cavitation" and this word emerged in the literature for the first time in 1895. One of the locations at which cavitation can take place is at ship propellers. The function of a ship was well below her design speed as observed in 1893 in the trials of the destroyer HMS Daring. The reason for the reduction of the speed is explained by the effect of initiation of water vapor bubbles on the blades. The same problem was visible after that in the first turbine ship, the Turbinia. The existence of the vapor bubbles on the ship's propellers lowers the thrust required to drive the ship. The higher power of the engine does not increase the propulsion of the vessel but leads to origination and preservation of the cavities. There are several experiments performed to study the cavitation phenomenon on propellers [106].

According to the classification scheme of Lauterborn [106, 53], there are four different kinds of cavitation inception: Hydrodynamic, Acoustic, Optic and Particle cavitation. Hydrodynamic cavitation is initiated by alteration of the pressure when the liquid is flowing. Acoustic cavitation occurs upon application of a sound field in the liquid. Optic cavitation is induced by focusing intense (laser) light in the liquid, and particle cavitation is originated by elementary particles. The tension in the liquid is responsible for hydrodynamic and acoustic cavitation, while the optic and particle cavitation are a result of local energy deposition.

If a sound wave is generated in a liquid with an amplitude that is large enough to bring the pressure below the vapor pressure in the negative parts of the sound wave profile, cavitation bubbles can be formed. They will oscillate in response to the applied sound field. These bubbles can be either stable cavities which oscillate for many periods of the acoustic field or be transient bubbles that exist just for less than one cycle of the sound field. The variation of the bubble size is not proportional to the amplitude of the pressure wave, since this is a nonlinear phenomenon. Due to the high compressibility of the gas inside the bubbles a large amount of potential energy is accumulated from the sound 
field during the bubble expansion. It is converted to kinetic energy while the bubble is collapsing [106].

When a gas bubble is trapped acoustically in a liquid and oscillates with large amplitudes in the presence of a sound field, the bubble oscillation may be accompanied by emission of visible light in each oscillation cycle. This phenomenon is called single-bubble sonoluminescence (SBSL). This light emission consists of very short pulses $(\leq 50 \mathrm{ps})$ emitted upon bubble collapse. From spectral analysis of the emitted light, the temperature of the gas which is responsible for this emission can be be higher than the temperature of the surface of the sun $\left(\approx 7,000^{\circ} \mathrm{C}\right)$ with speculations as high as a million degrees which could potentially lead to thermonuclear fusion [26]. Hiller et al. found that doping the liquid with a noble gas has a strong effect on the emission of light in SBSL [40]. Single-bubble sonoluminescence is a well-studied phenomenon $[64,34,60,63,71,57]$. A large number of theoretical explorations have been carried out $[30,37,58,6,15]$, one of them being the theory that the light is originating from an optically thin plasma core.

Cavitation damage is produced in the case that a bubble collapse occurs in the vicinity of a boundary. Due to the asymmetric collapse of the cavity, a high-speed liquid jet is developed which can penetrate into the neighboring interface and form pits. The cavitation erosion phenomenon has been studied in many details by several workers $[49,65,81,7,16,91,98,73,74]$. This fact can be considerable in cases like ship propellers, turbines, pumps and nozzles [73].

When a cavitation bubble is exposed to a shock wave, a high-speed liquid jet can be induced which concentrates a part of the shock wave energy into a small volume [73]. This phenomenon leads to creation of high values of pressures and temperatures locally [87]. The shock wave emitted from the forced bubble collapse is enhanced and the collapse time is reduced with respect to inertial cavity implosion [77]. The liquid jet develops in the direction of shock wave propagation. There are several applications in which these effects play a significant role. Some examples are shock wave lithotripsy (SWL), increasing the permeability of cell membranes by shock waves at low dosage, drug delivery, gene transfer, laser surgery e.g. ophthalmology, sonophoresis and revascularization $[108,73,105,101,36,69,96,86,47]$. In treatment by extracorporeal shock wave lithotripsy (ESWL), not only the miss-shot of shock focusing can produce tissue damage, but cavitation bubble collapses play an important role in destroying the tissue [87]. These cavitation bubbles originate in the focal region of the lithotripter shock wave because of the tension tail of the shock wave profile. They do not dissolve quickly after the shock wave passage. This is due to gas diffusion during their long expansion phase. Tissue damage can occur due to interaction between subsequent shock waves and the initiated cavitation bubbles [73]. The mechanism of tissue damage by cavitation bubbles during ESWL is also explored in [45].

The variety of applications of the shock wave-bubble interaction phenomenon confirms the importance of the investigation of this effect in a detailed proce- 
dure. In this work, the behavior of laser-generated single cavitation bubbles upon exposure to a lithotripter shock wave is studied. Laser generation of the bubbles provides the possibility of investigation of single bubbles which are well-controlled (i.e. in space and time) and being exposed to a shock wave. The forced bubble dynamics can be recorded by means of high-speed photography techniques. By systematic variation of the particular experimental parameters (shock wave amplitude and the time of the shock wave impact) the bubble dynamics under the influence of the shock has been studied in detail. 


\section{Chapter 2}

\section{Outline of previous investigations}

A spherical bubble prior to the exposure to a shock wave can be forced to collapse in a non-symmetric pattern after the moment of the shock wave impact. The breaking of the spherical symmetry of the flow field around the cavity leads to the induction of a high-speed liquid jet in the original direction of the shock wave propagation $[73,77]$.

For a collapsing bubble in vicinity of a boundary, the jet formation is suggested by Kornfeld and Suvorov in 1944 [49]. Later, in 1961, Bowden and Brunton [12] investigated experimentally the deformation of solid surfaces by the impact of a high-speed liquid cylinder or jet. Mader [62] in 1965 studied numerically the origination of detonation by the interaction of shocks with spherical voids. Experimental study of cavities and micro munro jets considering their role in explosion is performed by Bowden and McOnie in 1965 [13]. The initiation of microjets in liquids as a consequence of impact or shock wave was illustrated experimentally in 1966 by Bowden [11]. Shock wave-bubble interaction is also experimentally investigated in 1968 by Ellis et al. [31] and some other related experiments are carried out by Brunton et al. in 1968 [19], in 1974 by Chaudhri and Field [20] and by Lauterborn in 1972 and 1974 [51, 52]. Later, by applying a shock wave produced by a spark discharge, Tomita, Shima and other authors investigated the forced bubble collapse and jet induction $[93,91,94]$. Other discussions about the jet induced bubble collapse can be found in $[73,90,44]$.

One of the first experimental studies of the effect of shock waves on bubbles is performed in 1972 by Lauterborn [51]. He used Q-switched ruby laser pulses to focus the light in liquid such as water or silicone oils or other liquids, to initiate cavities via optical breakdown. Compression of small air bubbles near to the location of laser breakdown, due to the shock wave originated from this region is investigated. The experimental data are compared to the calculated results of Noltingk and Neppiras [67]. 


\section{Initial deformation velocity of a bubble exposed to a shock wave}

The term $2 u$ is introduced as the theoretical prediction of imparted velocity to the bubble wall as a result of the shock wave-bubble interaction, while $u$ being the fluid particle velocity behind the shock front (see equation (3.55)). However, the jet tip velocity of $2 u$ is lower than the experimental values. This is due to the fact that in equation (3.55) convergence and nonlinear effects are neglected. A numerical study of the impingement of a shock wave with an amplitude of $85 \mathrm{kbar}$ on a cavity in nitromethane is performed in 1965 by Mader [62]. At the moment of the shock wave arrival, the shock speed is 4500 $\mathrm{m} / \mathrm{s}$, and the particle velocity is $1710 \mathrm{~m} / \mathrm{s}$. Therefore, the free surface velocity becomes $3420 \mathrm{~m} / \mathrm{s}$. Due to convergence and shocks, the free surface velocity increases to $5200 \mathrm{~m} / \mathrm{s}$ at the moment of collapse of the cavity [62]. Then, the maximum jet velocity is higher by a factor of 1.5 than the free surface velocity $2 u$.

Dear and Field [28] presented experimental results related to the shock pressure of $2.6 \mathrm{kbar}$. They report a value of $2 u$ as $300 \mathrm{~m} / \mathrm{s}$, while the jet velocity being $\approx 400 \mathrm{~m} / \mathrm{s}$. The final jet velocity is increased by a factor of less than 1.5 times the term $2 u$. This is due to the application of a weaker shock. Therefore, the convergence and nonlinear effects are supposed to be less prominent compared to the results of Mader [62]. These jet velocities are able to produce damages. Pressures of $\approx 1 \mathrm{GPa}$ can be generated when the bubble collapse occurs close to a metal boundary.

The effect of bubble surface shape at the moment of shock wave impact on initial deformation is illustrated in [87], where experimental results of Tomita and his co-workers (1993) are illustrated. A rising air bubble is exposed to a shock wave with an amplitude of $65 \mathrm{MPa}$. The bubble aspect ratio is defined as $\chi=d_{x} / d_{y}$, where $d_{x}$ is the bubble diameter in the direction of shock wave propagation and $d_{y}$ being its diameter perpendicular to that. Bubble flatness is introduced as $f=\frac{d_{x}-d_{y}}{d_{x}}$ and is $f=0.23$ in this experiment. This is a relatively high value which indicates a distinguished deformation of the bubble surface. The deformation velocity of the bubble surface at $1 \mu$ s after the moment of the shock impingement is $103 \mathrm{~m} / \mathrm{s}$. This is 2.3 times larger than the particle velocity behind the impinging shock. The initial deformation velocities for nonspherical bubbles with $\chi>1$ are larger than those for spherical ones.

\section{Enhancement of bubble collapse by a shock wave}

A theoretical investigation of extracorporeal shock wave lithotripter (ESWL) induced cavitation is performed by Church [21]. At the focus position, the shock wave is modeled as the voltage response of a parallel $R L C$ circuit to a current impulse. The incident shock wave has a peak positive pressure of $P_{+}=1,000$ bars which is followed by a negative tail of $P_{-}=-160$ bars. Using numerical integration of the Gilmore-Akulichev formulation for bubble 
dynamics, the bubble radius vs. time curves are derived. A preexisting bubble with the size between 1 and $10 \mu \mathrm{m}$ is expanded by more than a factor of 100 from the initial size for $250 \mu \mathrm{s}$ in response to the negative pressures produced by the shock wave. The maximum cavity radius is $\approx 1400 \mu \mathrm{m}$. The collapse is very violent in which far $U V$ or soft $x$ - ray photon emissions (black body) may occur. Including the zeroth-order effect of gas diffusion, it is concluded that this effect does not produce a significant truncation of the amplitude of the primary collapse pressure. Nevertheless, it lowers the collapse temperature noticeably.

A theoretical study of shock wave-inertial microbubble interaction, is performed by considering three different lithotripter shock waves [109]. They are a modified XL-1 lithotripter, a Dornier HM-3 lithotripter and an electrohydraulic lithotripter with pressure-release reflectors. The bubble dynamics connected with the shock waves from a modified XL-1 lithotripter is simulated by using the Gilmore model coupled with zeroth-order gas diffusion. Lithotripter shock wave-microbubble interaction provides strong secondary shock wave emissions comparable to the shock waves emitted from inertial collapse of much larger cavities (in mm-size). Shortening of the tensile stress of the lithotripter shock wave can considerably reduce the large expansion of the bubbles which are exposed to the shock. This might lower the risk for vascular injury during shock wave exposure [109].

Interaction between a shock wave with amplitude of $1.9 \mathrm{GPa}$ and a cylindrical air cavity, is simulated by Ball et al. in 2000 [4]. They used a Free-Lagrange code for simulation. This method provides a good resolution at the bubble surface. Multiple reflections of shock waves in the cavity lead to heating and compression of the gas inside the bubble. They report the maximum temperature as $\sim 12,000 \mathrm{~K}$. The compression in not isentropic, and the assumption of spatially-uniform isentropic compression underestimates the pressure and temperature in the cavity during compression. They also predicted the position of hot gas at the final stage of the collapse, which correlates with the experiments investigating the luminescence in the cavities.

Laser-induced single cavitation bubbles are exposed to a lithotripter shock wave (LSW) investigating the influence of phase of bubble oscillation on enhancement of the collapse pressure and formation of microjets by Sankin et al. (2005) [77]. Maximum pressure amplification is achieved when the shock wave impinges on the cavity in its collapse phase of oscillation cycle. In this case, the forced collapse time fits to the duration of the positive part of the LSW profile. In this condition, maximum energy transfer from LSW to the bubble interior takes place. There is an optimal bubble size beyond which the tensile tail of LSW profile retards the bubble dynamics. In case that the cavity is smaller than this optimal size, there is not enough opportunity for transfer of momentum, while the duration of interaction decreases. In general, the forced collapse time of expanding bubbles is longer than that of collapsing cavities. This is due to the delay time which is needed for an expanding bubble to stop the ongoing expansion first, before the collapse occurs. 
Klaseboer et al. in 2007 [43] explored the interaction between lithotripter shock waves with a cavitation bubble by modeling the flow field around the bubble with boundary-element method (BEM). The results of this theoretical study are compared with the experimental data reported by Sankin et al. in 2005 [77]. The bubble radius at the moment of the shock wave impact is defined as $R_{S 0}$ and the maximum radius of the cavity is $R_{\max }=300 \mu \mathrm{m}$.

In response to the shock wave impingement, the entire bubble is displaced in the direction of the shock wave passage. The maximum reported displacement of the bubble centroid at the moment of the jet impact (i.e. $\approx 82 \mu \mathrm{m}$ ) is related to $R_{S 0} / R_{\max }=0.8$, for initially collapsing cavities. But in the case of initially expanding bubbles, the highest value of the bubble centroid displacement occurs at $R_{S 0} / R_{\max }=0.65$, which is $66 \mu \mathrm{m}$. The turning point is related to $R_{S 0} / R_{\max }=0.55$, where there is an inversion of the trends for smaller values of $R_{S 0} / R_{\max }$. In this case, at the moment of jet impingement, the displacement of the expanding bubbles is larger than that of collapsing cavities. This is due to the fact that the period of displacement for expanding bubbles is longer than that for collapsing ones [43].

Considering the results related to measured and water-hammer pressures, the jet and relative velocity, the kinetic energy and Kelvin impulse, all at the moment of jet impingement, it can be concluded that a maximum takes place in the case of intermediate values of $R_{S 0} / R_{\max }$, which is about 0.6 for initially collapsing bubbles and a bit less for initially expanding cavities. The bubble collapse time in the case of $R_{S 0} / R_{\max }=0.6$ for initially collapsing bubbles is $1.2 \mu \mathrm{s}$ while for initially expanding ones being $1.6 \mu \mathrm{s}$. As the duration of the positive part of the shock wave is about $1 \mu \mathrm{s}$, it can be said that the maximum takes place, if the whole compressive part of the shock wave interacts with the bubble. For lower values of $R_{S 0} / R_{\max }$, just a portion of the energy is transferred from the shock wave into the bubble interior, while for larger values of $R_{S 0} / R_{\text {max }}$, the tension tail of the shock profile reduces the effect of the positive part. The quantities like pressure and jet velocity, etc. are truncated for both cases of $R_{S 0} / R_{\max }$ being higher and lower that the intermediate value, which indicates a reduction of the collapse strength in these cases relative to intermediate values of $R_{S 0} / R_{\max }$ [43].

The bubble collapse time (i.e. half of the period of the oscillation cycle) is estimated as [43]:

$$
t_{\text {coll }} \sim R_{S 0} \sqrt{\rho / P_{s h}}
$$

in which $P_{s h}$ being the typical pressure that the bubble feels. As an initial guess for parameter $P_{s h}$, it is assumed to be equal to the peak pressure of the shock wave, $P_{s h}=39 \mathrm{MPa}$. By setting the value of $P_{s h}$ and $R_{S 0}=R_{\max }$ into equation (2.1), the collapse time will be $1.50 \mu \mathrm{s}$. But, the numerically derived value for collapse time is $3.52 \mu \mathrm{s}$. The discrepancy between these values is described considering the fact that in equation (2.1), $P_{s h}$ is assumed to be the pressure around the bubble during the entire collapse. Nevertheless, we know 
that actually the effective pressure is much less. A linear relation between $R_{S 0}$ and collapse time, is represented in equation (2.1) because the effect of the negative tail of the shock wave is not considered. This effect can be important for high values of $R_{S 0} / R_{\max }$, as the expansion wave tends to retard the collapse, and this linear relation can not hold anymore [43].

A very good agreement between the collapse times derived from the experiments and the ones derived from the model is reported. Also, comparison between peak pressure measurements because of the jet impingement and water-hammer model implies a good qualitative agreement. Even though the compressibility of the water around the cavity and the internal shock waves in the bubble are not taken into account in the applied model, there is a good agreement with experimental results, while the other effects are perhaps important just to the second order [43].

\section{Effect of intensity of the incident shock wave}

Calculated values of the bubble diameters in the direction of the shock wave passage as well as perpendicular to that as a function of time (i.e. $d_{x}$ and $d_{y}$, respectively), obtained by Masubuchi et al., are presented in [87]. For using a relatively weak incident shock $\left(P_{s h} / P_{0}=10\right)$, the bubble collapse occurs nearly spherical $\left(P_{s h}\right.$ is the shock wave pulse amplitude and $P_{0}$ the atmospheric pressure). The values of $d_{x}$ and $d_{y}$ correlate with the theoretical prediction of Rayleigh collapse [75]. Nevertheless, in the case of a relatively strong impinging shock $\left(P_{s h} / P_{0}=1,000\right)$, a significant deviation of the bubble surface from Rayleigh's spherical bubble collapse is observed.

Grove and Menikoff $[38,87]$ numerically investigated interaction between a planar shock wave and a cylindrical air cavity. For the case of weak shocks $\left(P_{s h} / P_{0}=100\right)$, the Mach number ahead of the shock is $M=U_{s h} / C=1.009$, where $C$ being the sound speed in water. At the moment of the shock impingement on the bubble, a pair of diffraction nodes is formed. The angle between incident shock and contact increases due to the curvature of the bubble, and finally each diffraction node is bifurcated into an anomalous reflection. Eventually, when the shock passes the opposite bubble wall, the two nodes originated by diffraction of the Mach line through the bubble interface coalesce, and the water shock wave disengages from the cavity. In the case of strong shock wave $\left(P_{s h} / P_{0}=10,000\right)$, the effects of nonlinearities of the flow are more important and the rarefaction spreads out over a larger distance. The contact surface is highly distorted, and during the collapse the pressure is increasing which finally induces a liquid jet that impinges on the back of the bubble.

The interaction of a planar shock with a bubble is investigated by Bourne and Field [10], applying a two-dimensional method. The shock pressure ranges from 0.3 to $3.5 \mathrm{GPa}$. Jet formation has been studied as well as light emission. Increasing the shock pressure above a critical value, the jet velocity exceeds the shock speed in the ambient medium. Collapse of bubbles in several geometries under the effect of a shock pressure amplitude of $0.3 \mathrm{GPa}$ have been considered 
to study the jet formation mechanism. This investigation indicates that the formation of the jet is strongly dependent on the initial geometry of the cavity.

The interaction between a shock wave and a gas cavity for weak and strong incident shocks is simulated by Ding and Gracewski (1996) using a finite volume method [29]. Weak shock waves are defined by the shock peak pressure being in the range $P_{s h} \leq 30 \mathrm{MPa}$ and strong shocks by the amplitudes being between 500 and 2,000 MPa. For weak shocks, it is pointed out that the Gilmore model supports a precise prediction of the bubble behavior. The deviation of the bubble surface from spherical shape is so small that a jet is not produced via the collapse of the gas void. But the jet is developed in the case of strong shock waves and then, the Gilmore model is not valid anymore. The jet penetration to the opposite cavity wall can have a velocity of as high as $2,000 \mathrm{~m} / \mathrm{s}$. The speed of the jet is dependent on the strength of the incident shock, but is nearly independent on the initial size of the gas cavity.

Ohl and Ikink (2003) [69] investigated the compression of spherical bubbles in water under the effect of a shock wave. The bubble sizes are between 7 and $55 \mu \mathrm{m}$. For bubbles with a size of a few microns, a liquid jet is developed in the direction of the shock wave passage, even for shock waves with peak pressures of $10 \mathrm{MPa}$. This fact is in contradiction with numerical results of Ding and Gracewski [29], in which there is no jetting for shock wave amplitude of 30 $\mathrm{MPa}$. Bubbles exposed to shock waves can be applied as microsyringes. In the case that biological cells are located in front of the shock wave induced jets, it is suggested that drugs may be delivered into them.

\section{Influence of pressure profile and impulse of the shock wave}

Tomita [87] explains the effect of the pressure profile on the maximum bubble wall pressure studied by Tomita et al. in 1986. Three different pressure impulses are considered. The rise time of the pressure profiles plays an important role in the maximum pressure values for small bubbles, while it is not significant for large cavities. Another considerable factor is the pressure impulse. For a specific amount of impulse, there is an optimal cavity size at which the most violent collapse takes place.

\section{Shock wave - bubble interaction near to a boundary}

Tomita and Shima [91] conducted an experiment examining impulsive pressure creation and damage pit formation, originating from cavity collapse. For a bubble located very close to a rigid interface, very high impulsive pressures are produced not only from the first collapse but also from the second collapse of a void. This is probably due to the collapse of tiny bubbles produced during the first collapse of the original cavity. In this case, shock wave-bubble interactions provide locally high impulsive pressures. An attached air bubble exposed to a 
shock wave creates a damage pit, which is due to the impact pressure of liquid microjet.

Interaction between lithotripter shock waves and air bubbles near to a thin plastic foil is investigated by Philipp et al. [73]. They produced shock waves by underwater spark discharge, and used an ellipsoidal reflector to reflect and focus the shock waves. The plastic foil with an air bubble on its lower side was located at the geometric focus of the experimental setup. The examined bubble radii range from 0.1 to $1.2 \mathrm{~mm}$. The pressure pulse amplitude in the geometric focus is about $65 \mathrm{MPa}$ followed by an expansion tail with minimum pressure of $-6 \mathrm{MPa}$. The single bubbles collapse aspherically because of the existence of both the shock and the boundary. For bubble initial radii of 0.15 to $1.2 \mathrm{~mm}$, the measured collapse time is between 1 and $9 \mu \mathrm{s}$. There is not a linear relation between collapse time and the initial bubble size. This is due to the pressure profile of the shock wave, in which the compression wave is followed by a stress wave. The shock wave induced jet penetrates the foil and produces a small hole. Philipp et al. [73] report generation of a hole with diameter of $80-300 \mu \mathrm{m}$ in the foils, for bubble initial radii in the range of 0.3 to $0.7 \mathrm{~mm}$. The velocity of the bubble wall at the moment that the shock hits the cavity, is $2 u=83 \mathrm{~m} / \mathrm{s}$. The jet velocity after collapse is larger than the value of $2 u$. The collapse jet velocity is by a factor of $(5-10)$ times the term $2 u$. Then, the collapse jet velocity averaged over $1 \mu \mathrm{s}$, is between 400 and $800 \mathrm{~m} / \mathrm{s}$, for the explored cavity sizes. The implosion jet speeds depend on the initial bubble size and the maximum shock pressure. Nevertheless, the speed of the jet averaged over $5 \mu$ s from the moment of collapse, ranges from almost zero up to $210 \mathrm{~m} / \mathrm{s}$, for initial bubble radii in the range of 0.1 and 0.9 mm. Philipp et al. [73] suggested an "optimum" initial cavity radius of 500 to $600 \mu \mathrm{m}$ which is related to the maximum jet velocity.

Tomita et al. [89] exploded a $10 \mathrm{mg}$ silver azide $\mathrm{AgN}$, pellet by illuminating it with a pulsed Nd:YAG laser with pulse duration of $7 \mathrm{~ns}$, to produce shock waves in water. The detonation takes place at one focal point of a semi-ellipsoidal reflector $\left(F_{1}\right)$. After reflections, the shock wave is focused at the second focal point of the reflector $\left(F_{2}\right)$. The expansion waves are also originated and being focused there. The focused shock wave has a positive peak pressure of 133 $\mathrm{MPa}$. Locating a $10 \mathrm{~mm}$ diameter steel sphere at the position of $F_{2}$ (instead of kidney stones), the shock wave-bubble interaction as well as bubble-bubble interaction, are investigated. Near to the steel surface, cavitation bubbles are produced and the reflected shock wave from the boundary, interacts with them. The collapse of these cavitation bubbles produces secondary shock waves. Considering two neighboring bubbles (with the distance of about $1 \mathrm{~mm}$ ), the shock wave emitted from collapse of one bubble can have an effect on the next one in a way that the collapse of the second bubble becomes destructively. The high pressure at the focus (i.e. $133 \mathrm{MPa}$ ) is able to disintegrate the kidney stones and gallbladder stones. Shock wave-bubble interaction has a prominent effect on tissue damage. High pressures originating from the interaction between shock waves and bubbles cause damage of human tissue more significantly than the 
focused high pressures. Then, shock wave-bubble interaction is an important factor in stone fragmentation and also has a prominent effect on tissue damage.

Kodama and Tomita [48] studied the collapse of laser-generated single cavitation bubbles in the vicinity of a gelatin surface, as well as the interaction between a shock wave and an air bubble attached to that surface. These issues are related to the behavior of in vivo cavitation bubbles and the succeeding tissue damage mechanism during intraocular surgery, intracorporeal and extracorporeal shock wave lithotripsy. The cavitation bubbles near to the gelatin surface tend to migrate away form it. Nevertheless, in this case liquid jet formation in the direction to the surface is not considerably observed. For a wide range of relative distances $\gamma=d / R_{\max }$, the period of the motion of cavitation bubbles is increased to longer than twice the Rayleigh collapse time, except for very small values of $\gamma$. Where, $d$ is the distance between the gelatin surface and the laser focus position and $R_{\max }$ is considered as the maximum bubble radius. In the case of studying shock wave-air bubble interaction, a liquid jet develops in the direction of the gelatin surface. The jet velocity is of the order of tens of $\mathrm{m} / \mathrm{s}$ at the gelatin surface. The impact pressure at the gelatin surface is tens of MPa and the penetration depth, a few $\mathrm{mm}$. The liquid jet is able to damage the gelatin surface.

An experimental exploration of the interaction between pressure waves and contrast agent bubbles near to cells, is performed by Wolfrum et al. (2002) [104]. The pressure waves are produced by an underwater spark discharge. The bubbles expand strongly and produce considerable damage to the cells via succeeding collapse, even in the case of low pressure amplitudes. The deformation of the cell near to a collapsing bubble is much more noticeable compared to that during the cavity expansion phase. For the jet developed from each bubble, there are three factors determining its direction, which are the vicinity to other cavities, the neighborhood of a solid boundary and the direction of the pressure wave. The jet is frequently developed away from the shock wave source via bubble collapse, in case that no other adjacent contrast agent bubbles exist.

Wolfrum et al. in 2003 [103] created shock waves via focusing of a Q-switched Nd:YAG laser pulse in water near to suspended salt crystals. Bubbles with different sizes are originated during slow dissolution of the salt crystals. Bubble migration toward boundaries and even into cracks or clefts are recorded. Boundaries are a petri dish and salt grains. The collapse of single bubbles in their vicinity is aspherical and the bubble shape upon implosion is complicated to some extent. Bubbles near to multiboundaries can be broken up or deformed with developing jets in the direction of each surface.

Sankin and Zhong in 2006 [78] examined the behavior of single laser-induced bubbles in the vicinity of an elastic boundary under interaction with a shock wave. Cavities with collapse times of $T_{c}=121 \mu$ s are used and the normalized shock wave arrival time is defined as $\alpha=t / T_{c}$, where $t$ represents the moment of shock wave impact with respect to the time of bubble formation. The normalized stand-off distance $\gamma$ is altered between 0.3 and 1.2. Penetration 
of the jet initiated from asymmetric bubble collapses near to the interface is investigated. For $\alpha=0.95$, the maximum jet penetration occurs in the case that the dimensionless distance is $\gamma=0.75$, while for $\alpha=0.50$, it takes place at $\gamma=0.79$. The penetration depth for shock wave-bubble interaction near to the moment of first collapse $(\alpha=0.95)$ is by a factor of two times larger than that at the moment of maximum bubble expansion $(\alpha=0.50)$. The effect of normalized shock wave arrival time $\alpha$ on penetration depth, resembles the effect on pressure enhancement from shock wave-bubble interaction in the absence of a boundary, studied in [77]. In the case that the bubble forced collapse time matches the duration of the shock wave compression phase, maximum penetration depth is provided.

The shock-induced implosion of a gas cavity in the focal region of a lithotripter shock wave close to a rigid interface is simulated numerically by Johnsen and Colonius in 2008 [42]. The effects of the initial stand-off distance, the pulse amplitude as well as the pulse width on the maximum wall pressure are investigated. This consideration provides estimation of the damage potential of shock-induced collapse in shock wave lithotripsy. The maximum pressure lowers with initial stand-off distance and pulse width and rises with pulse amplitude. For the cavities initially being near to the boundary, pressures on the wall on the order of $1 \mathrm{GPa}$ might be originated as a consequence of the shock-induced collapse.

\section{Multi-bubble configuration}

The behavior of bubbles attached to a solid boundary exposed to shock waves is examined by Tomita et al. in 1984 [92]. Shock waves are produced by an underwater spark discharge between tungsten electrodes. In the case of two bubbles attached to the wall and having a symmetric configuration, the maximum impulsive pressure falls off rapidly by decreasing $S / R_{e}$ in the range of $S / R_{e} \leq 4$. Here $S$ is the distance between the two bubbles, and $R_{e}=1$ $\mathrm{mm}$. This is due to a noticeable interaction between the two cavities which are exposed to the shock wave initiated from the spark discharge. If the two bubbles are far from each other, the bubble-bubble interaction becomes less prominent and the behavior of each bubble resembles that of a single bubble. In the interval of $S / R_{e} \geq 4$ there is no significant difference between the cases of single and two bubble configurations. Increasing the bubble-bubble interaction strength is accompanied by a longer collapse time. If the bubble number $N_{B}$ is increased, the collapse time rises, while the maximum pressure drops drastically at $N_{B}=2$. The orientation of the developed liquid jet is given by the shock wave direction, shock intensity, the interaction between bubbles, as well as the interaction between cavities and the wall.

The interaction between an underwater shock wave and two air bubbles attached to a gelatin surface is examined by Kodama et al. (1996) [46]. The distance between the two bubbles as well as the cavity diameters are altered. There is a critical value for the separation of the bubbles, beyond which the 
direction and depth of the liquid jet do not depend on the existence of the other cavity. The bubble collapse and penetration which it provides are intensified by the shock wave emitted from the collapse of the neighboring one.

The interaction between bubbles in a cavitation field, is studied numerically by Kuttruff in 1999 [50]. The behavior of single cavities under the influence of pressure waves emitted from their neighboring voids in a cavitation cloud is considered. The smaller bubbles collapse earlier in a sound field cycle. The interaction between bubbles leads to transfer of energy from smaller cavities to the larger ones. The larger bubbles have more time to accumulate energy from the smaller cavities. They implode later with more violent collapses. The strongest implosions are related to the bubbles collapsing latest in a sound cycle.

\section{Influence of surface curvature of an interface near to a bubble}

Theoretical and experimental investigations of the motion of cavitation bubbles in the vicinity of a curved rigid boundary are performed by Tomita et al. in 2002 [90]. They initiated single bubbles axisymmetrically by focusing a laser beam from the bottom of a bubble chamber through a mirror close to a curved wall. They considered the effect of surface curvature of the boundary. Using image theory, parameter $\xi$ is defined as $\xi>1$ for concave walls, $\xi<1$ for convex walls, and $\xi=1$ for flat walls. The stand-off distance $\gamma$ is defined as the ratio of the initial distance between the bubble and the boundary $d$ to the maximum bubble radius $R_{\max }\left(\gamma=\frac{d}{R_{\max }}\right)$. The prolongation coefficient of collapse time for the case of a curved boundary is defined as [90]:

$$
\mu=\frac{\xi^{2}}{\gamma(1+\xi)}
$$

The prolongation coefficient $\mu$ increases monotonically with $\xi$, therefore, for convex walls $(\xi<1)$, the period of the cavity motion is shorter compared to the case of flat boundaries $(\xi=1)$. However, a longer period is expected for concave walls $(\xi>1)$.

The cavity motion depends on both parameters $\xi$ and $\gamma$. The bubble dynamics is gradually retarded by increasing the parameter $\xi$. The growth of the bubble is almost spherically for the experiments with convex, concave and flat walls. But, the bubble shape develops differently in the collapse phase. A cone-shaped bubble is produced in the collapse period, for the case of convex boundaries $(\xi<1)$. Nevertheless, just a small deviation from the spherical geometry in the collapse period, is visible for the case for concave boundaries $(\xi>1)[90]$.

For the case of a spherical wall near to the bubble, the prolongation coefficient of collapse time is [90]: 


$$
\mu=\frac{a^{\prime}}{\gamma\left(2 a^{\prime}+\gamma\right)}-\frac{1}{a^{\prime}} \ln \left[\frac{\left(a^{\prime}+\gamma\right)^{2}}{\gamma\left(2 a^{\prime}+\gamma\right)}\right],
$$

where $a^{\prime}=a / R_{\text {max }}$, and $a$ represents the sphere radius. A dimensionless period of the bubble oscillation (i.e. growth and collapse) is introduced by Best and Blake [8] as:

$$
\tau_{p}=\frac{2}{\sqrt{6}}\left[B\left(\frac{5}{6}, \frac{1}{2}\right)+\frac{1}{2} \mu B\left(\frac{7}{6}, \frac{1}{2}\right)\right]=1.829(1+0.4065 \mu),
$$

while $B$ being the incomplete beta function. The first term on the right hand indicates the Rayleigh collapse of the bubble in an infinite medium, while the second term is related to the prolongation of the oscillation period due to existence of a boundary. As shown in equations (2.2) and (2.3), the prolongation coefficient $\mu$ is only dependent on the surface geometry of the wall. Defining the parameter $\lambda_{p}$ as the ratio between $\tau_{p}$ and the dimensionless period of a Rayleigh bubble collapse $\left(\tau_{p}=1.829\right)$, the theoretical expression is [90]:

$$
\lambda_{p}=1+0.4065 \mu
$$

By measuring the period of the bubble oscillation, Tomita et al. [90] found a good agreement between the theoretical prediction and the experimental data for the convex, concave, flat and sphere walls. By decreasing the parameter $\mu$ the value of $\lambda_{p}$ approaches to unity regardless of the wall surface geometry.

\section{Bubble dynamics in vicinity of a wall}

Vogel et al. (1989) investigated the dynamics of bubbles produced via optical breakdown in water in the vicinity of a rigid wall [98]. They applied the time-resolved particle image velocimetry (PIV) technique to measure the flow field velocity around the laser-generated bubbles. Agreement between derived pathlines and the results of numerical calculations is reported. After the first collapse, a ring vortex is formed for stand-off distance $\gamma>1.5$, while it is produced before the first collapse for $\gamma \approx 0.9$. A strong reduction in acoustic emission during collapse occurs in the case of ring vortex formation before the first bubble collapse. The connection between jet formation and ring vortex generation is discussed by Lauterborn and Bolle (1975) [54].

A theoretical investigation of shock wave propagations in elastic solids originated from cavitation microjet impact is performed by Zhong and Chuong in 1993 [107]. The presented model includes reflection and refraction of shock waves from a solid-water interface. The impact pressure at the rigid wall, the stress, strain and also the velocity discontinuities at the locations of the propagating shock fronts are calculated.

Ohl et al. [70] investigated the dynamics of acoustically driven cavitation bubbles as well as the laser-generated single bubbles in the vicinity of a rigid wall. The light emission from laser-induced bubbles relative to the sym- 
metry of cavity collapse is studied. The radius of the emission spot and the number of emitted photons as a function of bubble maximum radius are measured. For the stand-off distance of $\gamma \leq 3.5$ the light emission is so weak that it can not be distinguished from the dark signal of the applied ICCD (intensified CCD) camera.

Brujan et al. in 2001 [17] initiated cavitation bubbles with a laser in the vicinity of an elastic wall, in order to study the interaction of the bubbles with the boundary. They emphasized the effect of elastic modulus of the interface on the bubble dynamics. The elastic modulus $E$, is varied between $0.017 \mathrm{MPa}$ and $2.03 \mathrm{MPa}$. For each value of $E$, the dimensionless stand-off distance is varied in the range of $0<\gamma<2.2$. The maximum liquid jet velocity in the direction away from the interface ranges between $300 \mathrm{~m} / \mathrm{s}$ and $600 \mathrm{~m} / \mathrm{s}$. These values are dependent on the elastic modulus of the boundary. For the jet heading in the direction of the wall, the highest velocities are between $400 \mathrm{~m} / \mathrm{s}$ and 800 $\mathrm{m} / \mathrm{s}$ (averaged over $1 \mu \mathrm{s}$ ).

Experimental investigation of the interaction between cavitation bubbles and composite surfaces by Tomita and Kodama (2003) [88] suggests that the characteristics of composite surface and also the distance from the bubble to the boundary determine the cavity migration.

A Q-switched Nd:YAG laser beam has been used to generate single cavitation bubbles with maximum radius of $R_{\max }=1.5 \mathrm{~mm}$, by Lindau and Lauterborn in 2003 [59]. Bubble dynamics close to a boundary is studied and the shock wave scenario around the collapsing bubble is discussed. They report initiation of a counterjet for a stand-off distance $\gamma$ in the range of $1<\gamma<3$. This is in agreement with the results of Vogel et al. (1989) [98] which indicate that the counterjet formation disturbing the development of the principal jet is pronounced for $1 \leq \gamma \leq 2$. Rising of the counterjet is very fast and it is observable for a long time interval compared to the collapse time.

Interaction between an underwater detonation bubble and an immersed structure has been investigated both experimentally and numerically by Klaseboer et al. [44] in 2005. They used a coupled BEM-FEM approach (boundary element method and finite element method) for simulation of the interaction in three dimensions. The BEM method is applied for the fluid domain, while FEM method used for the structure domain.

\section{Liquid impact}

Lush [61] investigated the case when a liquid mass collides with a perfectly plastic solid, using a steady, normal and oblique shock configurations. Pressure and deformation induced from the liquid mass impact are studied. Considering the normal impact of a plane-ended cylinder of water on a solid boundary, for impact velocities below $100 \mathrm{~m} / \mathrm{s}$, a water-hammer formulation can give quite precise results for the generated stress. For higher impact velocities, a significantly higher stress is developed due to the alteration of the liquid bulk modulus. 
Field claims that the collision between a liquid drop and a rigid surface with a velocity of a few $100 \mathrm{~m} / \mathrm{s}$ produces pressures in the order of gigapascals with durations in the time scale of submicroseconds [33]. A jet is induced with velocity of several times the impact velocity. A review of research on the physics of liquid impact and shock wave-cavity interaction can be found in [33].

Dear and Field in 1988 [27] investigated the collision between a disk-shaped liquid gel layer and a metal slider. The drop has a radius of curvature of $11 \mathrm{~mm}$ and the velocity of impact is $150 \mathrm{~m} / \mathrm{s}$. Generation of a shock wave envelope is presented and jets with speed of $\approx 1800 \mathrm{~m} / \mathrm{s}$ can be developed. They also studied impact between a drop and a wedge. In the case that the contact angle $\beta$ is less than a critical value $\beta_{c}$, the contact point moves with a velocity higher than that of the shock wave. Then, the shock envelope is attached to the contact point. In this case, no jet is produced in the wedge. For contact angles $\beta>\beta_{c}$, the primary shock wave propagates faster than the contact edge. Therefore, the liquid surface is deformed and a jet is initiated. The value of the critical contact angle $\beta_{c}$ depends on the collision velocity and the shock wave speed in the liquid.

An experimental investigation of the phenomenon of liquid jet impact on the liquid and solid surfaces connected with cavitation damage and rain erosion, is performed by Obara et al. [68]. The impact of the liquid jet develops a compressive wave, which is joined with cavitation behavior and erosion mechanisms. 


\section{Chapter 3}

\section{Theory}

\subsection{Shock wave propagation}

To study the evolution of a pressure wave in a liquid, its equation of state i.e. the relation between e.g. pressure and density, has to be taken into account. The equation of state in a weakly compressible fluid, is known as the Tait equation:

$$
\frac{P+B}{P_{0}+B}=\left(\frac{\rho}{\rho_{0}}\right)^{\gamma}
$$

where $P$ is the pressure and $\rho$ the density of the medium. Parameters $P_{0}$ and $\rho_{0}$ are equilibrium pressure and density, while $\gamma$ is an empirical parameter. $B$ and $\gamma$ are both parameters that depend on the medium considered. For water, they are selected in a way to result in a correct value of $C_{0}=1483 \mathrm{~m} / \mathrm{s}$ for acoustic wave propagation velocity at room temperature. In this case, we have $B=295.5 \mathrm{MPa}$ and $\gamma=7.44$ [76].

In general, the pressure $P$ is a function of density $\rho$ and entropy per mass $s$. The density deviation of $\rho-\rho_{0}$ is related to the pressure deviation of $P-P_{0}$ by a Taylor series expansion of the equation of state at constant entropy $s=s_{0}$ [32]:

$$
P-P_{0}=a\left(\frac{\rho-\rho_{0}}{\rho_{0}}\right)+\frac{b}{2 !}\left(\frac{\rho-\rho_{0}}{\rho_{0}}\right)^{2}+\ldots,
$$

where the parameters $a$ and $b$ are defined as:

$$
\begin{gathered}
a=\rho_{0}\left[\left(\frac{\partial P}{\partial \rho}\right)_{s}\right]_{\rho=\rho_{0}} \\
\mathrm{~b}=\rho_{0}^{2}\left[\left(\frac{\partial^{2} P}{\partial \rho^{2}}\right)_{s}\right]_{\rho=\rho_{0}} .
\end{gathered}
$$

For the case of the Tait eq. (3.1) one obtains: 


$$
\frac{b}{a}=\gamma-1
$$

The size of the ratio $\frac{b}{a}$ determines the finite-amplitude wave distortion in the propagating medium. If the deviation in density relative to $\rho_{0}$ is small $\left(\frac{\rho-\rho_{0}}{\rho_{0}} \ll 1\right)$ one can ignore the second and higher terms in the series expansion (3.2). It reduces to the following relation:

$$
P-P_{0}=\left[\left(\frac{\partial P}{\partial \rho}\right)_{s}\right]_{\rho=\rho_{0}}\left(\rho-\rho_{0}\right) .
$$

The small-amplitude sound velocity in the liquid is given as:

$$
C_{0}=\sqrt{\left[\left(\frac{\partial P}{\partial \rho}\right)_{s}\right]_{\rho=\rho_{0}}} .
$$

The equation for conservation of mass (continuity) is:

$$
\frac{\partial \rho}{\partial t}+\nabla \cdot(\rho \mathbf{u})=0
$$

where parameter $\overrightarrow{\mathbf{u}}$ is the particle velocity in the medium. The equation expressing the conservation of momentum reads:

$$
\rho\left(\frac{\partial}{\partial t}+\mathbf{u} \cdot \nabla\right) \mathbf{u}+\nabla P=0
$$

By using both conservation equations (eq. (3.7) and eq. (3.8)) as well as equation (3.5), under the assumption that the particle velocity and relative change in density are very small (higher order terms are neglected), the linear wave equation is obtained as:

$$
\frac{1}{C_{0}^{2}} \frac{\partial^{2} P}{\partial t^{2}}-\nabla^{2} P=0
$$

The wave propagation velocity $C_{0}$ is a constant for given $P_{0}$ and $s_{0}$. This assumption holds just for small amplitude waves that produce very tiny changes in the density of the liquid. If the amplitude of the pressure wave increases, the higher order terms of eq. (3.2) should be taken into account. In this case, the sound velocity in the disturbed medium gives:

$$
C=\sqrt{\left(\frac{\partial P}{\partial \rho}\right)_{s}} .
$$

The relation between sound velocity regarding nonlinearities $C$ and the sound speed in the linear case $C_{0}$ is [32]:

$$
C=C_{0}+\frac{b}{2 a} u=C_{0}+\frac{\gamma-1}{2} u
$$


and for a plane wave, the propagation speed which belongs to particle velocity $u$ will be [32]:

$$
\left[\frac{d x}{d t}\right]_{u}=C_{0}+\beta u=C+u
$$

in which $\beta=\frac{\gamma+1}{2}$. As the velocity $C$ is increasing with $\rho$, the points on the profile of a finite-amplitude wave that have a larger density (higher pressure) move a longer distance compared to the points with lower pressures at a given time. Therefore the wave profile becomes distorted. The longer the wave travels, the more distortion occurs and the wave front steepens. In this way, shock waves can be produced. Shock waves can only be explained by taking into account the nonlinearity of the equation of state of the medium.

Neglecting the dissipation terms can lead to good approximations in many cases. In the region where dissipation becomes very small, the shock front can be considered as a thin layer. But the gradients get large so that the wave front can be assumed as a discontinuity for certain physical parameters such as pressure and density. So, the idealized equations will hold on both sides of the shock front but not within it. As the fluid velocity tangential to the shock remains continuous, we consider a plane shock wave propagating in one direction (i.e. $x$ ) and the flow velocity in the same direction. If we consider a fixed control volume in a given inertial frame with two fixed endpoints as $x_{1}$ and $x_{2}$ (see Figure 3.1), conservation of mass leads to [39]:

$$
\frac{d}{d t} \int_{x_{1}}^{x_{2}} \rho d x=(\rho u)_{x_{1}}-(\rho u)_{x_{2}}
$$

where $(\rho u)_{x_{1}}$ is the rate of mass inflow at position $x_{1}$ and $(\rho u)_{x_{2}}$ is the rate of mass flowing out at point $x_{2}$. Then, the left side of equation (3.13) corresponds to the time rate of change of mass in the volume.

Considering the endpoints $x_{1}$ and $x_{2}$, the rate per unit area at which the

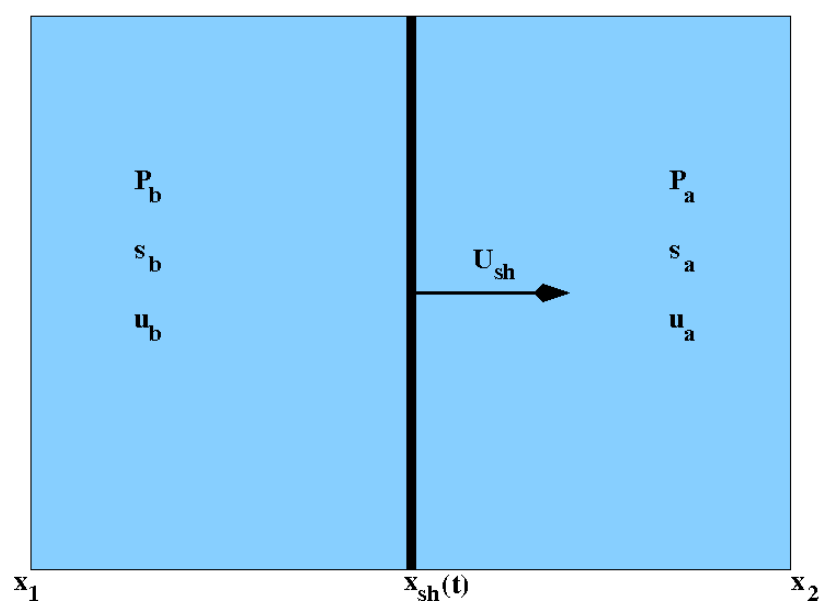

Figure 3.1: A schematic view of the propagation of the shock wave front [39]. 
momentum flows into the control volume is $(\rho u)_{x_{1}}(u)_{x_{1}}$ and the momentum outflow rate per unit area is $(\rho u)_{x_{2}}(u)_{x_{2}}$. The time rate of change of momentum in the control volume is equal to the rate of momentum inflow (per unit area) minus the rate of momentum outflow (per unit area) plus the net force (per unit area) which acts on the control volume. By neglecting the viscosity, this net force is $P_{x_{1}}-P_{x_{2}}$, then [39]:

$$
\frac{d}{d t} \int_{x_{1}}^{x_{2}} \rho u d x=\left(\rho u^{2}+P\right)_{x_{1}}-\left(\rho u^{2}+P\right)_{x_{2}} .
$$

Now, let us consider the change in energy. If the term $\frac{1}{2} \rho u^{2}$ is defined as kinetic energy per unit volume and $e$ as internal energy per unit mass, the energy density within the fluid is $\frac{1}{2} \rho u^{2}+\rho e$. The time rate of change of energy in the control volume by neglecting dissipation, is equal to the convective rate of inflow $\left(\frac{1}{2} \rho u^{2}+\rho e\right)_{x_{1}}(u)_{x_{1}}$ minus the outflow $\left(\frac{1}{2} \rho u^{2}+\rho e\right)_{x_{2}}(u)_{x_{2}}$, plus the work which is done by external pressures on the control volume, then:

$$
\frac{d}{d t} \int_{x_{1}}^{x_{2}} \rho\left(\frac{1}{2} u^{2}+e\right) d x=\left[\left(\frac{1}{2} \rho u^{2}+\rho e+P\right) u\right]_{x_{1}}-\left[\left(\frac{1}{2} \rho u^{2}+\rho e+P\right) u\right]_{x_{2}}
$$

Now, we direct attention to the plane wave discontinuity propagating with the velocity of $U_{s h}=d x_{s h} / d t$ between the two points $x_{1}$ and $x_{2}$, as illustrated in Figure 3.1. At the position of the shock front $\left(x_{s h}\right)$, the variables pressure $P$, particle velocity $u$, density $\rho$ and internal energy per unit mass $e$ are discontinuous. Splitting the integral in equation 3.13 into integrals from $x_{1}$ to $x_{s h}$ and from $x_{s h}$ to $x_{2}$, leads to:

$$
\frac{d}{d t} \int_{x_{1}}^{x_{2}} \rho d x=\left(\rho_{b}-\rho_{a}\right) U_{s h}+\int_{x_{1}}^{x_{s h^{-}}} \frac{\partial \rho}{\partial t} d x+\int_{x_{s h^{+}}}^{x_{2}} \frac{\partial \rho}{\partial t} d x,
$$

where $\rho_{b}$ and $\rho_{a}$ are the values of density on $-x$ and $+x$ sides of the shock front (letters $a$ and $b$ represent parameters ahead of and behind). If we assume that the points $x_{1}$ and $x_{2}$ are very close to $x_{s h}$, the integrals on the right will be negligible, and $(\rho u)_{x_{1}} \rightarrow(\rho u)_{b},(\rho u)_{x_{2}} \rightarrow(\rho u)_{a}$, then equation (3.13) gives:

$$
\left[\rho\left(u-U_{s h}\right)\right]_{a}=\left[\rho\left(u-U_{s h}\right)\right]_{b} .
$$

In a similar procedure, equations (3.14) and (3.15) yield:

$$
\begin{array}{r}
{\left[\rho u\left(u-U_{s h}\right)+P\right]_{a}=\left[\rho u\left(u-U_{s h}\right)+P\right]_{b}} \\
{\left[\rho\left(\frac{1}{2} u^{2}+e\right)\left(u-U_{s h}\right)+P u\right]_{a}=\left[\rho\left(\frac{1}{2} u^{2}+e\right)\left(u-U_{s h}\right)+P u\right]_{b}}
\end{array} .
$$

Equations (3.17) to (3.19) are called Rankine-Hugoniot relations [39].

Assuming the shock wave propagation in an undisturbed medium $\left([u]_{a}=0\right.$, $\rho_{a}=\rho_{0}$ and $\left.e_{a}=e_{0}\right)$, we set $[u]_{b}=u, P_{b}=P, \rho_{b}=\rho$ and $e_{b}=e$, equations 
(3.17) to (3.19) can be simplified as:

$$
\begin{array}{r}
\rho\left(U_{s h}-u\right)=\rho_{0} U_{s h}, \\
P-P_{0}=\rho_{0} U_{s h} u, \\
e-e_{0}=\frac{1}{2}\left(P+P_{0}\right)\left(\frac{1}{\rho_{0}}-\frac{1}{\rho}\right)
\end{array}
$$

By knowing the equation of state for the fluid, the increase in internal energy can be derived as a function of pressures $P$ and $P_{0}$ and densities $\rho$ and $\rho_{0}$ [22].

The velocity of the shock front $U_{s h}$ can be expressed as a function of pressure and density ahead and behind the shock, by considering equations (3.20) and (3.21), this gives [22]:

$$
U_{s h}=\sqrt{\left(\frac{\rho}{\rho_{0}}\right) \frac{P-P_{0}}{\rho-\rho_{0}}},
$$

and the particle velocity is:

$$
u=\frac{\rho-\rho_{0}}{\rho} U_{s h} .
$$

Substituting the density $\rho$ from equation of state (3.1), into equation (3.23), leads to [102]:

$$
U_{s h}=\sqrt{\frac{P-P_{0}}{\rho_{0}\left(1-\left(\frac{P+B}{P_{0}+B}\right)^{\frac{-1}{\gamma}}\right)}},
$$

which is the relation between shock front velocity and the pressure only. In a similar manner, the particle velocity can be derived as:

$$
u=\sqrt{\frac{P-P_{0}}{\rho_{0}}\left(1-\left(\frac{P+B}{P_{0}+B}\right)^{\frac{-1}{\gamma}}\right)} .
$$

The sound velocity $\left(C=\sqrt{\left(\frac{\partial p}{\partial \rho}\right)_{s}}\right)$ as a function of pressure can be derived using the Tait equation of state of the medium, equation (3.1) and reads [102]:

$$
C=\sqrt{\frac{\gamma}{\rho_{0}}\left(P_{0}+B\right)\left(\frac{P+B}{P_{0}+B}\right)^{\frac{\gamma-1}{\gamma}}} .
$$

Figure 3.2 depicts the relation between pressure $P$ and particle velocity $u$, as well as the shock velocity $U_{s h}$ (the Tait equation parameters $\gamma=7.44$ and $B=295.5 \mathrm{MPa}$ are taken from [76]). 


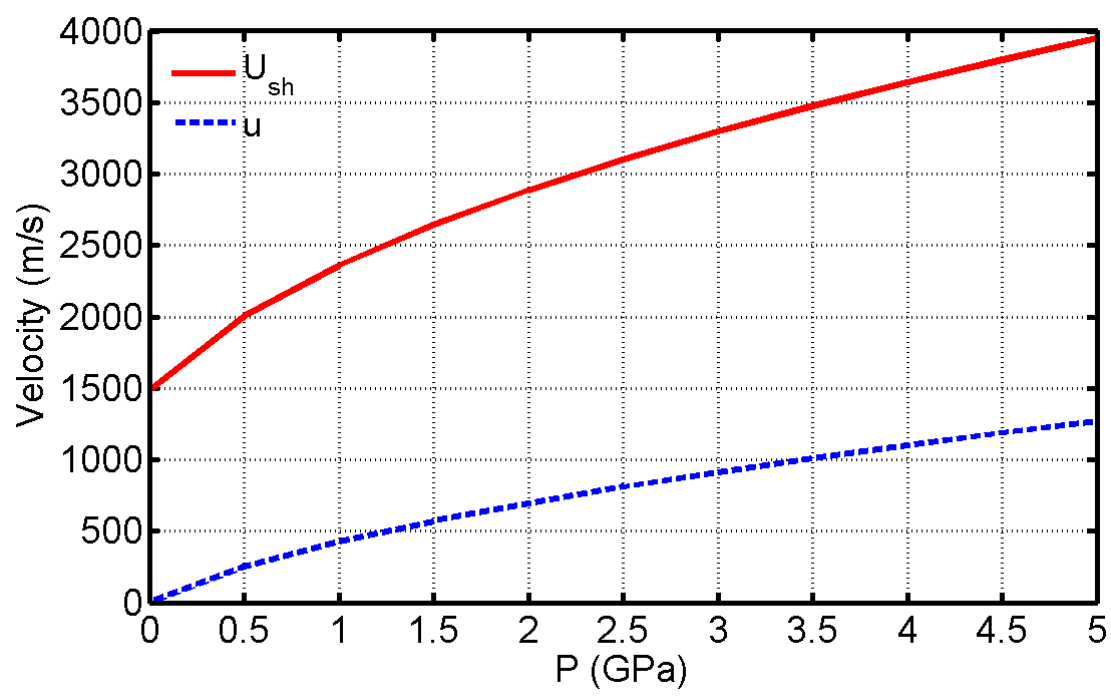

Figure 3.2: Velocity of the shock front $U_{s h}$ and particle speed $u$ as a function of pressure $P$, calculated using equations 3.25 and 3.26.

\subsubsection{Simulation of pressure wave propagation}

The acoustic wave passage in $1 D$ (i.e. $x$ direction) was simulated using the finite element method (FEM). Equations regarding conservation of mass and momentum (i.e. eq. (3.7) and eq. (3.8)) are solved ignoring dissipation terms. We also need a third equation to complete the solution, which is the relation between density and pressure. Therefore, the equation of state of water, the Tait equation (eq. (3.1)) with the static pressure of $P_{0}=0.1 \mathrm{MPa}$ and equilibrium density of $\rho_{0}=998 \mathrm{~kg} / \mathrm{m}^{3}$ at temperature of $20^{\circ} \mathrm{C}$ has been used. The Tait equation parameters $\gamma=7.44$ and $B=295.5 \mathrm{MPa}$ are obtained from [76].

A sine pressure wave $(S)$ as an initial condition is applied at point $x=0$ depicted in the left side of the graphs in Figure 3.3. The period of the initiated pressure waves at this point is $300 \mu \mathrm{s}$, and the amplitude ranges from 10 to $30 \mathrm{MPa}$. As the propagation velocity is a function of density and pressure, the wave steepens after traveling some distance from the source (at $x=0$ ).

In order to compare the signal with the linear case, a sine function $\left(S_{l}\right)$ with the same wavelength and amplitude as the source is plotted for the whole domain. The period of this wave is kept constant. The peak pressure points, due to higher propagation speed, tend to overtake the linear signal and the wave profile becomes steep. The negative peak is left behind the linear signal due to lower pressure and density and then, the lower propagation speed. The difference between the linear signal $S_{l}$ and the original wave $S$ is rising with increasing the distance from the source.

The waves steepen more as they travel to the right and the profile becomes finally discontinuous, which produces shock waves. Comparing the three different wave amplitudes in Figure 3.3, it is clear that the steepening occurs at 
a closer distance from the source, for the case of higher amplitudes of the pressure wave. The deviation from the linear signal is also more pronounced in the case of higher pressure amplitudes. This result indicates that the nonlinearity of the pressure wave propagation in a medium like water has a significant effect when increasing the pulse amplitude.
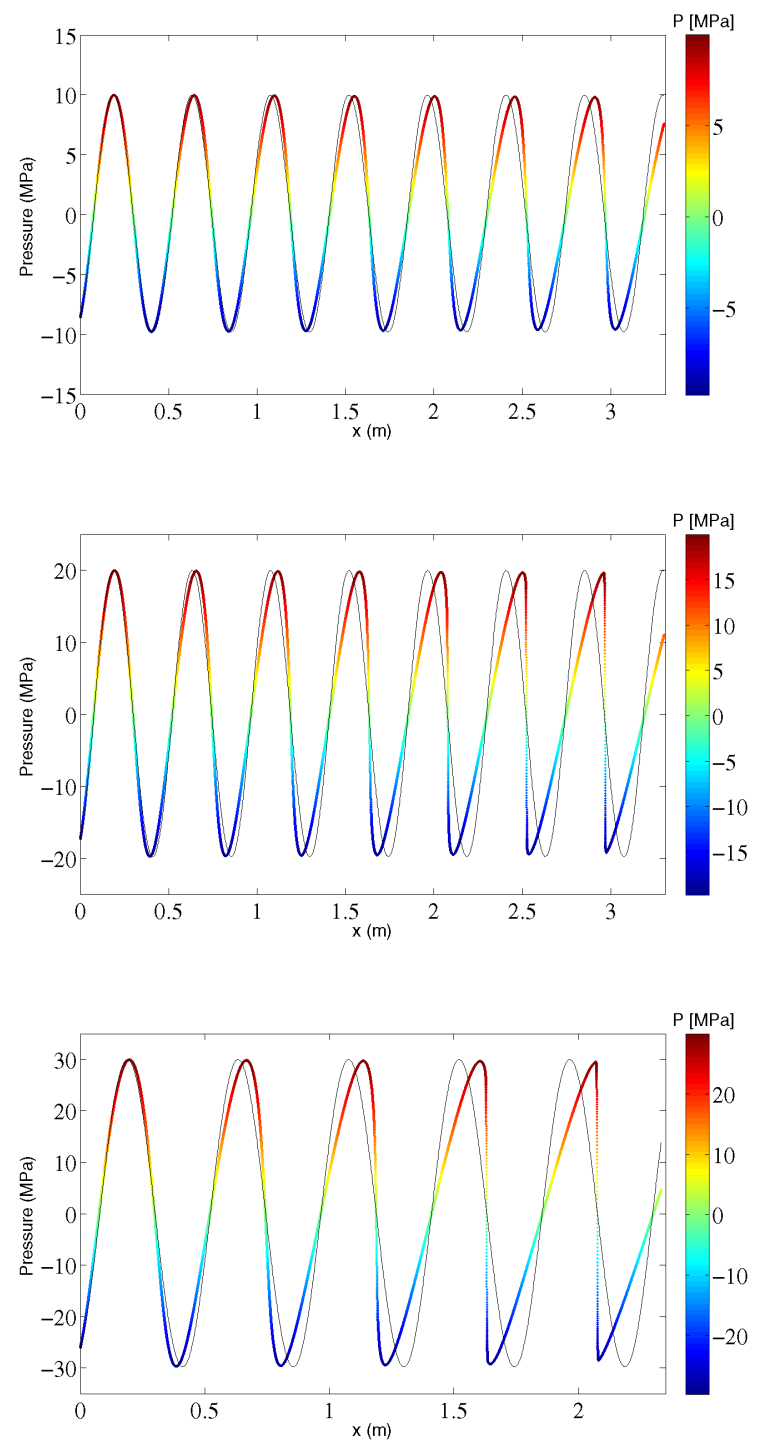

Figure 3.3: Nonlinear propagation of a pressure wave $(S)$ in water. The amplitudes of the initial pressure waves (at point $x=0$ ) from top to bottom are $P=10, P=20$ and $P=30 \mathrm{MPa}$. The black graphs are asymptotic linear propagation signals $\left(S_{l}\right)$. 
The initial (peak) acoustic Mach number is defined as [55]:

$$
M=\frac{u_{a}}{C_{0}},
$$

where $u_{a}$ is the maximum fluid velocity at the source position (at $x=0$ ). The wave number is:

$$
k=\frac{\omega}{C_{0}},
$$

in which $\omega=2 \pi / T$, and $T$ being the period of the sound wave.

The shock distance $D_{s h}$, is defined as the distance in which the wave develops a vertical tangent, which is the beginning of the shock wave initiation. It is given by [55]:

$$
D_{s h}=\frac{1}{\beta M k},
$$

where $\beta=\frac{\gamma+1}{2}$. With the period of the pressure wave at the source position being $T=300 \mu \mathrm{s}$, the shock distances for the pressure pulse amplitudes $P=$ 10,20 and $30 \mathrm{MPa}$ are $D_{s h} \approx 3.76,1.89$ and $1.27 \mathrm{~m}$. These values are in agreement with the results of the simulation which are plotted in Figure 3.3. 


\subsubsection{Reflection of shock waves}

When a shock wave impinges on a boundary, several scenarios depending on the angle of incidence and other physical parameters are possible. Here, a brief discussion of different types of shock wave reflections for finite amplitude waves is given.

1. Normal reflection at a solid interface

If the density variations are negligible, the pressure at the boundary is twice the incident pressure as a result of reflection of the shock wave from the surface. In the more general case, when the change in density is taken into account and constant entropy density in the water is assumed, the ratio of reflected to incident pressures as a function of densities is:

$$
R=\frac{P^{\prime}-P_{0}}{P-P_{0}}=\frac{\left(\frac{\rho^{\prime}}{\rho_{0}}\right)^{\gamma}-1}{\left(\frac{\rho}{\rho_{0}}\right)^{\gamma}-1},
$$

in which $\gamma$ is an empirical parameter in the Tait equation of state. Parameters $P$ and $\rho$ represent the pressure and density behind the incident wave front, while $P^{\prime}$ and $\rho^{\prime}$ are pressure and density behind the front of the reflected wave. The pressure increase by normal reflection at the rigid boundary is always larger than two, while for weak shock waves it approaches this value. Even for relatively immense pressures, the deviations from acoustic "pressure doubling" are not so significant [22].

2. Oblique incidence at a solid surface

Consider a plane compression shock front $(C I)$ with the speed of $U_{s h}$, incident on a rigid boundary $(A B)$ at point $C$ as illustrated in Figure 3.4. The shock wave is propagating in an undisturbed medium, and the flow velocity behind the incident shock front $(C I)$ will be $u$. The incident angle relative to the boundary is defined as $\alpha$. Therefore, the component of the fluid velocity normal to the boundary at point $C$ is $u \cos (\alpha)$. Reminding the assumption of a rigid boundary, a second shock front $(C R)$ should originate from point $C$ to produce a normal flow velocity component equal and in opposite direction to the term $u \cos (\alpha)$, then:

$$
u \cos (\alpha)+u^{\prime} \cos \left(\alpha^{\prime}\right)=0
$$

where $u^{\prime}$ is the flow velocity originating from the passage of the reflected shock wave $C R$, and the angle $\alpha^{\prime}$ is the reflection angle relative to the interface. As $\alpha$ approaches $90^{\circ}$, for any supposed angle $\alpha^{\prime}$, the normal component of the fluid velocity to the shock front $C R$ and ahead of that, becomes increasingly large. Therefore, the shock front $C R$ can be considered as propagating in a fluid under compression with a velocity away from that. In this case, the reflected shock wave velocity $U_{s h}^{\prime}$ becomes increasingly supersonic. 


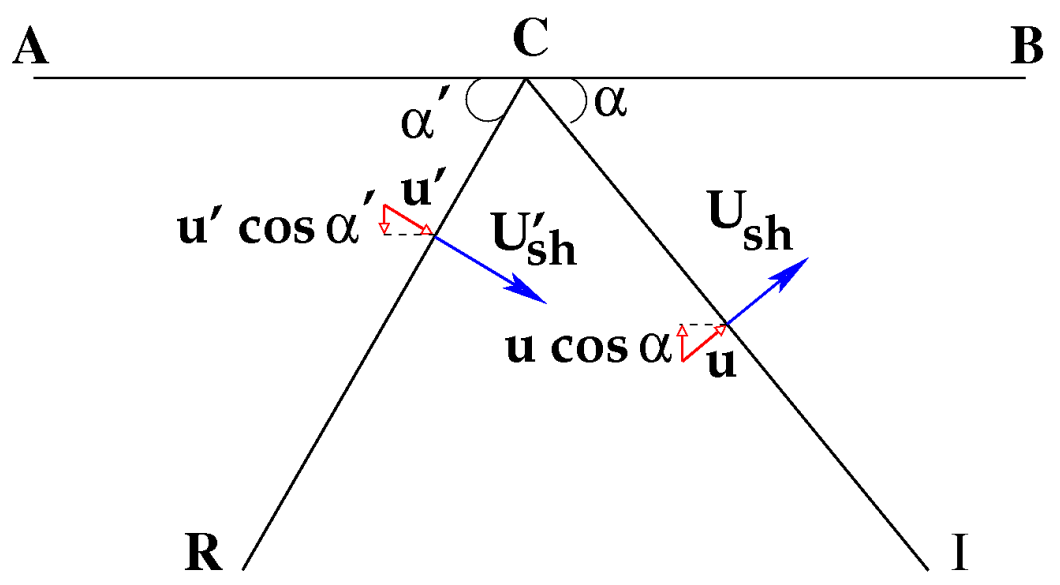

Figure 3.4: Regular reflection of a shock wave from a rigid boundary [22].

The tangential fluid velocity behind the incident shock front $C I$, is:

$$
u_{x}=\frac{\left(U_{s h}-u\right)+u \cos ^{2}(\alpha)}{\sin (\alpha)}=\frac{\left(U_{s h}^{\prime}-u^{\prime}\right)+u^{\prime} \cos ^{2}\left(\alpha^{\prime}\right)}{\sin \left(\alpha^{\prime}\right)}
$$

Equations (3.32) and (3.33) can be used to derive $U_{s h}^{\prime}-u^{\prime}$ and $\alpha^{\prime}$ for given values of $U_{s h}-u$ and $\alpha$. Basically, for a given intensity of the incident shock pressure $P$ and its direction, there are two values for $\alpha^{\prime}$. Then, there are also two values for the reflected shock pressure $P^{\prime}$ [22].

Just one of the two values of $\alpha^{\prime}$ has a physical meaning. The larger value of $\alpha^{\prime}$ is always related to a higher pressure discontinuity and therefore, corresponds to a stronger shock compared to the other smaller value. The smaller value of $\alpha^{\prime}$ corresponds to a weaker shock front. It is expected that the smaller $\alpha^{\prime}$ is physically realized in which the energy is deteriorated to a higher rate. The experimental data confirm or at least do not oppose this consideration. This kind of reflection as shown in Figure 3.4 is called regular reflection. The angle $\alpha^{\prime}$ is always larger than $\alpha$ [22]. By rising the value of $\alpha$, the mentioned two solutions coalesce into one, and there is no real solution for $\alpha^{\prime}$ in the case of more oblique incidence and larger $\alpha$. This fact implies that the simple geometry depicted in Figure 3.4 does not hold any more and another pattern of the shock fronts has to be considered. At this condition, the regular reflection is modulated and then it is called irregular reflection [22].

Let us define the auxiliary variable $\xi^{\prime}$ as:

$$
\xi^{\prime}=\frac{P^{\prime}+B_{0}}{P_{0}+B_{0}},
$$

where $B_{0}$ is a parameter in the Tait equation of state.

The angle of incidence in which transition from regular to irregular reflection occurs, is called $\alpha_{\text {extr }}$ and will be: 


$$
\tan ^{2}\left(\alpha_{e x t r}\right)=\frac{1}{\xi^{\frac{1}{\gamma}}} \cdot \frac{\gamma \xi^{\prime}\left(\xi^{\frac{1}{\gamma}}-1\right)+\left(\xi^{\prime}-1\right)}{\gamma \xi^{\prime}\left(\xi^{\frac{1}{\gamma}}-1\right)-\left(\xi^{\prime}-1\right)} .
$$

The intensity of the reflected wave as illustrated by $\xi^{\prime}$, rises noticeably at considerably large values of $\alpha$ and $P / P_{0}$. For instance, for the case $P=258.53 \mathrm{MPa}$ and incidence angle of $\alpha=40^{\circ}$, the pressure behind the reflected shock $P^{\prime}$ is $775.58 \mathrm{MPa}$ or three times the incident pressure, which corresponds to $\xi^{\prime}=2.0$. Table 3.1 shows the pressure increase in the case of normal and oblique reflections at a rigid interface as a function of incident pressure. It is shown that the pressure increase is larger for oblique reflection in all cases [22].

Table 3.1: Pressure increase at a solid boundary for normal and oblique reflections (from calculations of Polachek and Seeger) [22].

\begin{tabular}{ccccc}
\hline \hline $\begin{array}{c}\text { Incident pressure } \\
(\mathrm{MPa})\end{array}$ & $\begin{array}{c}\text { Reflected pressure } \\
(\text { Normal })\end{array}$ & $\begin{array}{c}\text { Reflected pressure } \\
\text { (Extreme) }\end{array}$ & $\alpha_{\text {extr }}$ & $\alpha_{\text {extr }}^{\prime}$ \\
\hline 16.13 & 2.03 & 2.99 & $71^{\circ}$ & $80.5^{\circ}$ \\
64.80 & 2.11 & 3.05 & $57^{\circ}$ & $73^{\circ}$ \\
258.53 & 2.35 & 3.61 & $40.5^{\circ}$ & $64^{\circ}$ \\
1013.42 & 2.92 & 4.63 & $28.5^{\circ}$ & $57.5^{\circ}$ \\
\hline
\end{tabular}

3. Mach reflection

If the incident shock is sufficiently oblique that the regular reflection can not fulfill the physical conditions, the regular reflection breaks down and there are large difficulties in the theoretical description. Nevertheless, experiments indicate that the incident and reflected shock fronts intersect at a point in the fluid off the boundary (not on the surface as in regular reflection) and a third shock front is produced by joining of the two waves which is extending to the rigid boundary and known as Mach stem. Thus, only one shock front, the Mach stem passes the fluid near to the wall, while the fluid sufficiently far away from the boundary or axis of symmetry is passed by two shock fronts. The velocity of these two parts of fluid must be in the same direction and the pressures also be the same. But due to the difference in past histories, other physical parameters, especially their density and amplitude of velocity are different. Then, a density discontinuity is initiated, called "slipstream", which is not a shock wave. This kind of reflection is called irregular or Mach reflection and is shown in Figure 3.5 [22].

The experimental results illustrating the Mach reflection can be found in chapter 4. In this work, we use shock waves which are focused and the Mach stem at the position of the focus is visible. 


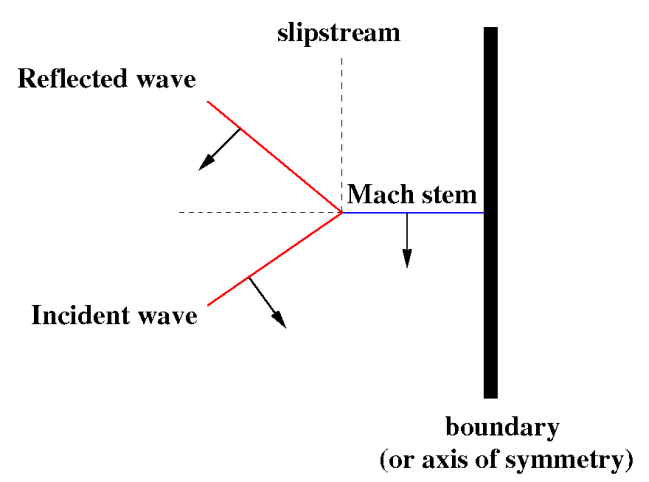

Figure 3.5: Mach reflection of a shock wave at a rigid surface [22].

\subsection{Spherical bubble dynamics}

Figure 3.6 displays schematically a spherical bubble in an infinite liquid. Assuming there is no boundary or shock wave or other sources of perturbation like buoyancy forces, the cavity may oscillate spherically [43]. The density of the liquid $\rho$ is supposed to be constant although the compressibility of the liquid at the moment of cavity collapse can have a significant effect. It is also assumed that the bubble contents are homogeneous and temperature $T_{B}$ and pressure $P_{B}$ of the cavity interior are uniform [14]. The fluid motion around the bubble is described by the velocity potential $\phi$, which satisfies the Laplace equation [43]:

$$
\nabla^{2} \phi=0
$$

and the fluid velocity is obtained by:

$$
\mathbf{u}=\nabla \phi
$$

Considering the conservation of mass, in the absence of mass transfer across the interface, the radial outward velocity of the fluid $u(r, t)$ at distance of $r$ from the bubble center will be [14]:

$$
u(r, t)=\frac{R^{2}}{r^{2}} \frac{d R}{d t}
$$

where $R(t)$ is the bubble radius at time $t$. For the velocity potential this yields [43]:

$$
\phi=-\frac{R^{2}}{r} \frac{d R}{d t} .
$$

The Navier-Stokes equation of motion for a Newtonian fluid is [14]:

$$
-\frac{1}{\rho} \frac{\partial P}{\partial r}=\frac{\partial u}{\partial t}+u \frac{\partial u}{\partial r}-\nu_{L}\left[\frac{1}{r^{2}} \frac{\partial}{\partial r}\left(r^{2} \frac{\partial u}{\partial r}\right)-\frac{2 u}{r^{2}}\right]
$$




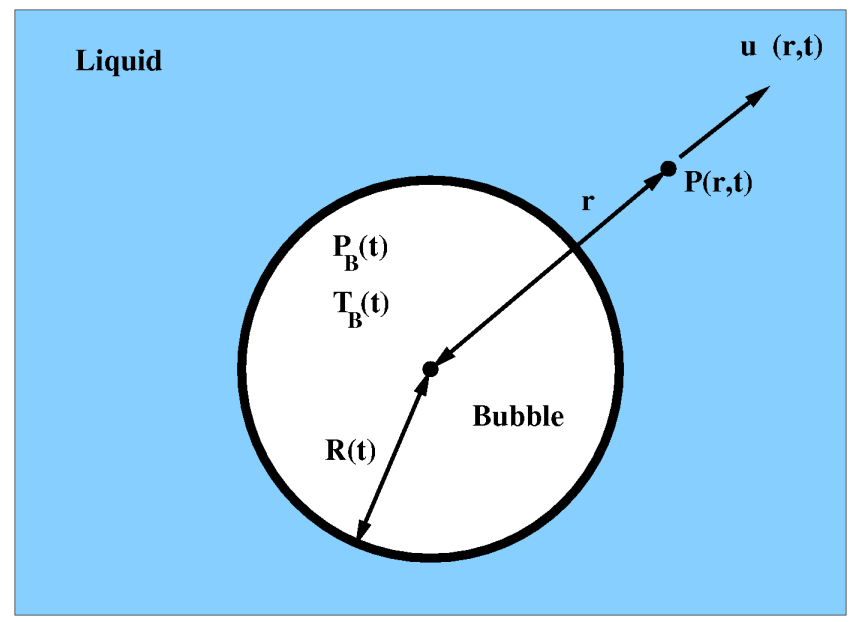

Figure 3.6: Schematic illustration of a spherical bubble in an infinite medium [14].

where $\nu_{L}$ being the kinematic viscosity of the liquid and the quantity $P$ is the pressure in the medium at a distance of $r$ from the cavity center. By substituting the velocity $u(r, t)$ from equation (3.38) into equation (3.40), one obtains:

$$
-\frac{1}{\rho} \frac{\partial P}{\partial r}=\frac{1}{r^{2}} \frac{d F}{d t}-2 \frac{F^{2}}{r^{5}}
$$

with $F(t)=R^{2} \frac{d R}{d t}$. It is worth mentioning that the viscous term has disappeared in this equation. By integrating equation (3.41) we have:

$$
\frac{P-P_{\infty}}{\rho}=\frac{1}{r} \frac{d F}{d t}-\frac{1}{2} \frac{F^{2}}{r^{4}}
$$

where $P_{\infty}$ is the pressure at infinite distance from the bubble center.

Ignoring evaporation or condensation (mass transport across the bubble wall), the net force on an assumed lamina on the bubble surface in the radially outward direction per unit area is zero. Thus:

$$
P_{B}=P_{r=R}+\frac{4 \mu_{L}}{R} \frac{d R}{d t}+\frac{2 S}{R}
$$

where $\mu_{L}$ and $S$ are dynamic viscosity and surface tension of the liquid, respectively. Substituting the value of $P_{r=R}$ from equation (3.42) and considering the relation $F(t)=R^{2} \frac{d R}{d t}$, one gets [14]:

$$
\frac{P_{B}(t)-P_{\infty}(t)}{\rho}=R \frac{d^{2} R}{d t^{2}}+\frac{3}{2}\left(\frac{d R}{d t}\right)^{2}+\frac{4 \nu_{L}}{R} \frac{d R}{d t}+\frac{2 S}{\rho R}
$$

This equation is called the generalized Rayleigh-Plesset equation for bubble dynamics. It can be seen that the viscous term is related to the boundary condition at the bubble wall [14]. 
By ignoring surface tension and viscosity effects, the equation (3.44) reduces to:

$$
\frac{P_{B}(t)-P_{\infty}(t)}{\rho}=R \frac{d^{2} R}{d t^{2}}+\frac{3}{2}\left(\frac{d R}{d t}\right)^{2} .
$$

This equation was first derived by Rayleigh (1917) [14].

The pressure inside the bubble $\left(P_{B}\right)$ is the sum of gas pressure $P_{G}$ and vapor pressure $P_{v}$. The major effect is related to the gas pressure. Neglecting the vapor pressure $\left(P_{B} \approx P_{G}\right)$ and assuming an adiabatic behavior of the gas content of the cavity, the internal pressure becomes [43]:

$$
P_{B}=P_{B 0}\left(\frac{R_{0}}{R}\right)^{3 \gamma}=P_{B 0}\left(\frac{V_{0}}{V}\right)^{\gamma},
$$

where $P_{B 0}$ is the bubble inner gas pressure at initial radius $R_{0}$ (i.e. at $t=0$ ), and $V$ represents the cavity volume. The parameter $V_{0}$ is the initial bubble volume $\left(V_{0}=(4 / 3) \pi R_{0}^{3}\right)$ and $\gamma$ is the ratio of specific heats of the bubble contents. An analytical solution of equation (3.45) is [14, 43]:

$$
\frac{3}{2}\left(\frac{d R}{d t}\right)^{2}=-\frac{P_{\infty}}{\rho}\left[1-\frac{R_{o}^{3}}{R^{3}}\right]+\frac{P_{B 0}}{\rho(1-\gamma)}\left[\frac{R_{0}^{3 \gamma}}{R^{3 \gamma}}-\frac{R_{0}^{3}}{R^{3}}\right] .
$$

The effects of viscosity and surface tension have been neglected. This is basically a relation between $d R / d t$ and $R$. At initial radius of $R=R_{0}$, the initial velocity $d R / d t$ is zero. At the time when the bubble is at its maximum size $\left(R=R_{\max }\right)$, the velocity $d R / d t$ also drops to zero. Therefore, we have [43]:

$$
\frac{R_{o}^{3}}{R_{\max }^{3}}-1=\frac{P_{B 0}}{P_{\infty}(\gamma-1)}\left(\frac{R_{o}^{3 \gamma}}{R_{\max }^{3 \gamma}}-\frac{R_{o}^{3}}{R_{\max }^{3}}\right) .
$$

This equation can be used to calculate $R_{0}$ numerically, in the case that the other parameters are given.

The Rayleigh-Plesset equation can be solved numerically to derive radius time (R-t) curves for bubble dynamics in an undisturbed medium. In this case, the parameters such as bubble collapse time and maximum cavity radius can be calculated.

The bubble oscillation starts with the radius $R_{0}$ and pressure $P_{B 0}$. The cavity inner pressure falls below the atmospheric pressure during expansion to the maximum size. In the absence of energy dissipation, the bubble collapses to its original size, and the pressure recovers to its initial value $P_{B 0}$. For explosion bubbles the value of $P_{B 0}$ is between 100 and 500 bar [22, 43].

The compressibility of the liquid must be considered for quick cavity collapse. Acoustic approximation is the simplest step in which a constant sound velocity $C$ is assumed. This assumption holds just in the case where the bubble wall velocity $d R / d t$ is always small relative to the sound speed in the liquid, $C$. Then, the loss of energy is taken into account by sound radiation. By neglecting surface tension and viscosity, the following equation provides the acoustic 
approximation, as Flynn shows [106]:

$$
R \ddot{R}+\frac{3}{2} \dot{R}^{2}=\frac{1}{\rho}\left[P_{L}+\frac{R}{C}\left(1-\frac{\dot{R}}{C}\right) \frac{d P_{L}}{d t}-P_{\infty}\right],
$$

where $\dot{R}=\frac{d R}{d t}$ is the bubble wall velocity and $\ddot{R}=\frac{d^{2} R}{d t^{2}}$ its acceleration. The quantity $P_{L}$ is the pressure on the liquid side of the bubble surface.

A more accurate statement regarding energy storage via compression of the liquid and also sound radiation, is given by Herring [106]:

$$
\left(1-\frac{2 \dot{R}}{C}\right) R \ddot{R}+\frac{3}{2}\left(1-\frac{4}{3} \frac{\dot{R}}{C}\right) \dot{R}^{2}=\frac{1}{\rho}\left[P_{L}+\frac{R}{C}\left(1-\frac{\dot{R}}{C}\right) \frac{d P_{L}}{d t}-P_{\infty}\right]
$$

Shock wave formation in the case that the bubble wall speed is near to the sound velocity, is investigated by Gilmore using the Kirkwood-Bethe approximation. This approximation implies that the propagation speed of the wave is equal to the sound velocity plus the fluid velocity. It gives [106]:

$$
\left(1-\frac{\dot{R}}{C}\right) R \ddot{R}+\frac{3}{2}\left(1-\frac{\dot{R}}{3 C}\right) \dot{R}^{2}=\left(1+\frac{\dot{R}}{C}\right) H+\frac{R}{C}\left(1-\frac{\dot{R}}{C}\right) \frac{d H}{d t}
$$

where the parameter $C$ is the sound velocity at the bubble wall. $H$ is the liquid enthalpy difference between the cavity surface and infinity, which is defined as [56]:

$$
H=\int_{\left.P\right|_{r \rightarrow \infty}}^{\left.P\right|_{r=R}} \frac{d P(\rho)}{\rho} .
$$

In the case that the water is regarded as incompressible, the bubble oscillation is not damped and has a constant period. Nevertheless, by considering the water as slightly compressible, damped oscillations with reducing periods are predicted by Keller and Kolodner [106]. 


\subsection{Shock wave - bubble interaction}

In this step, the case that a planar shock wave impinges on an initially spherical cavitation bubble, is considered. The velocity potential at the bubble surface with radius $R_{S 0}$ is derived by substituting $d R / d t$ from equation (3.47) to equation (3.39) and setting $r=R=R_{S 0}$ :

$$
\phi\left(R_{S 0}\right)= \pm R_{S 0} \sqrt{\frac{2 P_{\infty}}{3 \rho}\left(\frac{R_{0}^{3}}{R_{S 0}^{3}}-1\right)-\frac{2 P_{B 0}}{3 \rho(\gamma-1)}\left(\frac{R_{o}^{3 \gamma}}{R_{S 0}^{3 \gamma}}-\frac{R_{o}^{3}}{R_{S 0}^{3}}\right)}
$$

where $R_{S 0}$ is the bubble radius at the moment of the shock wave impingement. The negative values of the velocity potential correspond to expanding bubbles, while the positive ones are related to the collapsing cavities. A strength parameter in bubble dynamics if defined as:

$$
\epsilon=\frac{P_{B 0}}{P_{\infty}} .
$$

However, the influence of the dimensionless parameter $\epsilon$ on the bubble dynamics in shock wave-bubble interaction is insignificant [43].

The geometry of the interaction between a planar shock wave and a single cavitation bubble is depicted in Figure 3.7. The shock front propagates from bottom to the top ( $z$ direction) with a constant velocity of $U_{s h}$. At the moment of shock wave impact, the bubble can be in the expansion or collapse phase.

The ratio between acoustic impedances of water and air $R$ is large (i.e. $R \approx$ 3500). Therefore, when a shock wave hits the bubble, a part of the incident wave is reflected at the cavity surface as a tension wave. The reflected expansion wave propagates in the opposite direction of the original incident shock wave. This shock wave reflection transfers momentum to the cavity surface which accelerates the bubble wall and provides a pressure gradient across the bubble.
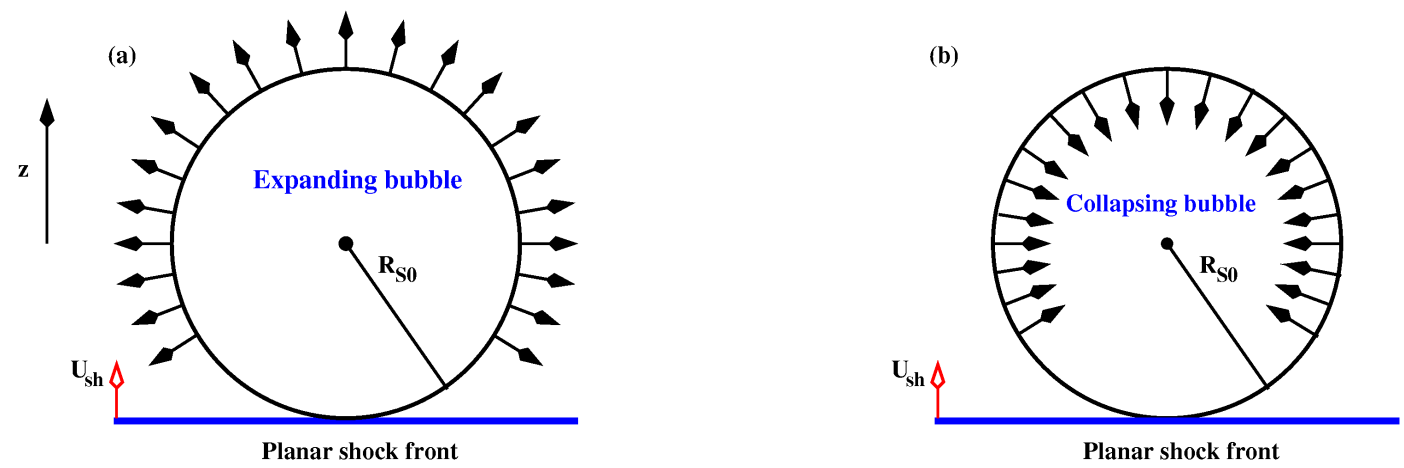

Figure 3.7: Schematic illustration of interaction between a planar shock wave and an initially spherical cavitation bubble. The shock wave propagates upwards with speed of $U_{s h}$. The bubble radius at the moment of shock impact is $R_{S 0}$ [43]. 
The spherical symmetry around the cavity collapse is disturbed as a response to the shock wave impact. In the final phase of the bubble collapse, a liquid jet is induced in the direction of the shock wave propagation [87].

A sketch of the interaction between a planar shock wave and a circular cavity is depicted in Figure 3.8 [28]. The incident shock wave $S$, is reflected at the free liquid surface. A corner wave $C$, and a reflected stress wave $R$, are initiated. Using linear shock wave theory, the velocity imparted to the free surface which is dependent on the angle $\alpha$ will be $[28,87]$ :

$$
V_{f}=2 u \sin \alpha
$$

while the parameter $u$ is the particle velocity behind the incident shock wave. Therefore, the velocity of initial deformation of the bubble surface on the axis of symmetry (i.e. at $\alpha=90^{\circ}$ in Figure 3.8) is twice the particle velocity behind the shock front. The relation between the particle velocity $u$ and the pressure shock wave amplitude $P$ is given by equation (3.26).

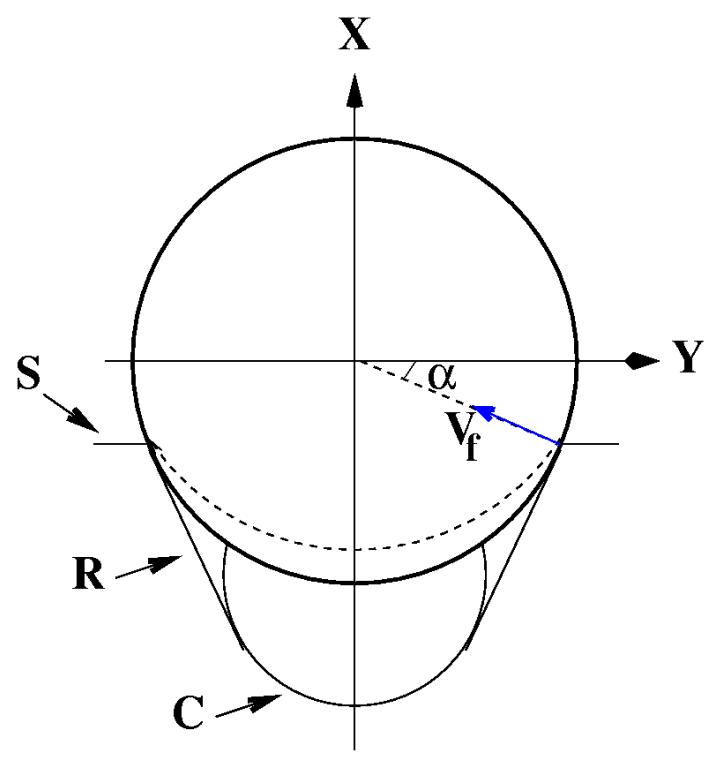

Figure 3.8: Schematic view of the interaction between a shock wave and a circular void [28].

We assume that the description of the fluid flow around the bubble by the velocity potential $\phi$ during the shock wave - bubble interaction holds in spite of existence of the shock wave. Considering the potential theory, the unsteady Bernoulli equation everywhere in the fluid as well as on the bubble wall reads [43]:

$$
P_{B}=P(z, t)-\rho \frac{D \phi}{D t}+\frac{1}{2} \rho|\mathbf{u}|^{2},
$$

where $P_{B}$ is given by equation (3.46), $P(z, t)$ is the far-field reference pressure and $D / D t$ is the material derivative $\left(D / D t=\frac{\partial}{\partial t}+\mathbf{u} \cdot \nabla\right)$. The bubbles considered by Klaseboer et al. [43] have small sizes $(<1 \mathrm{~mm})$ and collapse times 
$(<4 \mu \mathrm{s})$, thus buoyancy is ignored. Surface tension effects are also neglected, because of being very small compared to shock wave and hydrostatic pressures, for the bubble sizes which are explored.

The modeled pressure pulse traveling in $z$-direction has a speed of $U_{s h}=$ $1500 \mathrm{~m} / \mathrm{s}$. For numerical simulation, Klaseboer et al. [43] applied a smoothed signal of the experimental data in the absence of the single bubbles (similar to the pressure pulses measured in the free field in this work). The positive peak pressure is $39 \mathrm{MPa}$ with duration of almost $1 \mu \mathrm{s}$, while the succeeding negative pressure pulse amplitude being $-8 \mathrm{MPa}$ which has duration of about $2 \mu \mathrm{s}$. The far-field reference pressure term $P(z, t)$ is:

$$
P(z, t)=P\left(t-\frac{z+R_{S 0}}{U_{s h}}\right) .
$$

The moment of $t=0$ corresponds to the time that the shock wave impinges on the bubble. The initial conditions regarding the potential of the cavity wall (at $t=0)$ are given by equation (3.53), which are dependent on the bubble radius at this moment, $R_{S 0}$.

Prior to the shock impact, the velocity potential on the bubble wall $\phi\left(\mathrm{R}_{S 0}\right)$, is spherically symmetric. After the shock wave impingement on the bubble, the spherical symmetry is broken. Therefore, the velocity potential over the bubble surface $\phi$ is not uniform anymore. An integral solution of the Laplace equation applying boundary-element method (BEM) reads [9, 43]:

$$
c(x) \phi(x)+\int_{S} \phi(y) \frac{\partial G(y, x)}{\partial n} d S=\int_{S} G(y, x) \frac{\partial \phi(y)}{\partial n} d S .
$$

Parameter $c(x)$ is the solid angle viewed from the fluid at a position $x$ on the bubble surface $S$, and $G(y, x)=\frac{1}{|x-y|}$ is the free space Green's function. $\frac{\partial \phi}{\partial n}$ is the normal velocity at the cavity surface:

$$
\frac{\partial \phi}{\partial n}=\mathbf{n} \cdot \nabla \phi=\mathbf{n} \cdot \mathbf{u}
$$

The integration vector is $y$ and the integrations are performed at the bubble surface $S$. Note that at the cavity surface, the velocity potential $\phi$ is connected to the normal velocity $\partial \phi / \partial n$ there. Using the boundary-element method provides a possibility to derive the normal derivative of the potential in the case that the potential is known on the entire surface $S$.

Klaseboer et al. [43] solved equation (3.58) and derived the velocity vector $\overrightarrow{\mathbf{u}}$ at the bubble surface, by calculating tangential and normal velocities. The calculation is done using equation (3.58) together with equations (3.46) and (3.56), until the shock induced jet collides with the opposite bubble wall.

They compared the results of simulation using boundary-element formulation with experimental data of Sankin et al. (2005) [77]. For similar initial bubble sizes $R_{S 0} / R_{\max }$ (at the moment of shock wave impact), the collapse time of the initially expanding bubbles is longer than that for initially collapsing cavities. The maximum bubble radius is $R_{\max }=300 \mu \mathrm{m}$. 


\subsubsection{Jet velocity and impact pressure}

When a shock hits the bubble, the spherical symmetry around the cavity is broken and energy is transferred to the bubble interior. A liquid jet develops from the lower bubble wall in the direction of the shock wave passage. The upper bubble wall (i.e. on the side opposite to where the shock impinges) is also moving with a velocity which can not be ignored. For instance, in the case of $R_{S 0} / R_{\max }=0.6$, the speed of the opposite cavity wall at the moment of surface collision, $v_{o p p}$ is $475 \mathrm{~m} / \mathrm{s}$ (i.e. when the bubble is collapsing at the moment of the shock wave impact). Then, the relative velocity $v_{r e l}$ is given by:

$$
v_{r e l}=v_{j e t}+v_{o p p}
$$

where $v_{j e t}$ is the jet velocity. Positive values of $v_{\text {opp }}$ are relative to the case that the bubble upper wall displaces inwards. The parameter $v_{\text {opp }}$ is negative if the upper surface of the cavity is re-expanding at the moment of jet impingement.

Using the concept of water-hammer pressure $P_{W H, 0}$ [18], a relation between relative velocity $v_{r e l}$ and the shock wave pressure originated from the jet impingement can be established [43]:

$$
P_{W H, 0}=\rho U_{s h} v_{r e l} / 2 \text {. }
$$

Assuming a decay of impact pressure as $1 / r$, the peak pressure at the position of $r$ reads:

$$
P_{W H}=\frac{R_{\text {jet }}}{2 r} \rho U_{\text {sh }} v_{r e l},
$$

where parameter $R_{\text {jet }}$ represents radius of the jet at the time of surface impact. The maximum numerical value of pressure at the location of the jet impingement, is estimated as $1290 \mathrm{MPa}$, which relates to $R_{S 0} / R_{\max }=0.5$. This value corresponds to the bubbles which are collapsing at the moment of the shock impact [43].

\subsubsection{Kelvin impulse and kinetic energy}

In this step, we define the physical parameters which are difficult or even impossible to be experimentally determined. The Kelvin impulse vector $K$ is defined as [72, 43]:

$$
K=\rho \int_{S} \phi n d S
$$

where $\phi$ is the potential on the bubble surface $S$ and $n$ represents the normal vector at this surface. For a spherical bubble oscillation, the potential $\phi$ over the bubble surface is uniform and $\int_{S} n d S=0$. Then, the Kelvin impulse vector, $K$ drops to zero. Thus, the value of the Kelvin impulse vector is related to the rate at which the bubble departs from spherical symmetry. Strong shock waves produce larger values of $K$ and result in more deviation from 
spherical symmetry. The Kelvin impulse vector is usually in the direction of the jet propagation. Higher values of $K$ correspond to a higher jet velocity or a broader jet [43]. The dimensionless Kelvin impulse is defined as:

$$
K^{\prime}=\frac{K}{R_{\max }^{3} \sqrt{\rho P_{\infty}}}
$$

For all values of $R_{S 0} / R_{\max }$, initially collapsing cavitation bubbles lead to higher values for parameter $K^{\prime}$, with the exclusion of a tiny region with low values of $R_{S 0} / R_{\max }$.

Setting the parameter $\epsilon=100$ (see equation (3.54)), Klaseboer et al. [43] derived the dimensionless Kelvin impulse $K^{\prime}$ for different values of $R_{S 0} / R_{\max }$, numerically. They report the maximum value of $K^{\prime}$ which is related to $R_{S 0} / R_{\max }=$ 0.7 for initially collapsing cavities. The parameter $K^{\prime}$ is very small for low values of $R_{S 0} / R_{\max }$. There is a correlation between experimental data regarding pressure profiles [77] and the parameters as relative velocity, water-hammer pressure and Kelvin impulse, calculated in [43].

The kinetic energy of the liquid is introduced as [43]:

$$
E=\frac{\rho}{2} \int_{V}|u|^{2} d V=\frac{\rho}{2} \int_{S} \phi \frac{\partial \phi}{\partial n} d S .
$$

In order to convert the integral over the whole fluid domain $V$ to an integral over the bubble surface $S$, the Gauss theorem has been used [43]. The dimensionless kinetic energy is given by:

$$
E^{\prime}=\frac{E}{R_{\max }^{3} P_{\infty}}
$$

The energy of an oscillating bubble system prior to the jet impingement is:

$$
E_{0} \approx \frac{4}{3} \pi R_{\text {max }}^{3} P_{\infty}
$$

Then, in this case the dimensionless energy will be $\approx 4.2$ [43]. The maximum value of dimensionless kinetic energy of the cavity at the moment of jet impact is reported as $\approx 140$. Therefore, the shock wave-bubble interaction leads to an enhancement of the kinetic energy at the moment of jet impact up to a factor of almost 30 times the initial available energy. Klaseboer et al. [43] suggest a correlation between the behavior of the dimensionless parameters $E^{\prime}$ and $K^{\prime}$, where dividing the dimensionless kinetic energy $E^{\prime}$ by 140 , it fits within $5 \%$ to the values regarding the dimensionless Kelvin impulse $K^{\prime}$. 


\section{Chapter 4 Shock wave characterization}

\subsection{Generation of lithotripter shock waves}

There are different ways of generation of shock waves in a liquid, which all have one similarity, that is using a high voltage produced by a rapid capacitor discharge through an electroacoustic transducer [1]. This very fast capacitor discharge provides a quick conversion of electric energy to a high amount of acoustic wave energy. A brief description of these methods is given in the following:

1. Electrohydraulic system

An ellipsoidal reflector is applied using two underwater electrodes placed at focal point $F_{1}$ shown in Figure 4.1 . A high voltage $(\approx 20 \mathrm{kV})$ is applied between the two spark electrodes. The electric discharge rapidly evaporates the liquid. A spherical shock wave is built up at the position of $F_{1}$. By partial reflection from the ellipsoidal surface, the shock wave will be focused at focal point $F_{2}$ displayed in Figure 4.1 (where, e.g. kidney stones can be placed.) [1, 102].

2. Electromagnetic lithotripter

A strong pulsed current is sent into a flat coil, leading to a rapid change in the magnetic field. Eddy currents are induced in the metal membrane located at the top of the coil that create a magnetic counterfield. Thus, the membrane will be pushed away from the coil and produces plane pressure waves. In order to focus the pulse, a lens is required as displayed in Figure 4.1 [1, 102].

3. Piezoelectric system

Piezoelectric elements quickly expand in response to an applied high voltage due to the piezoelectric effect. Thus, they can produce pressure pulses in the liquid. Using a spherical segment of mosaic design of piezo elements, it is possible to focus the pressure waves without application of an acoustic lens. This system is applied in the present work, discussed in the next chapter. 

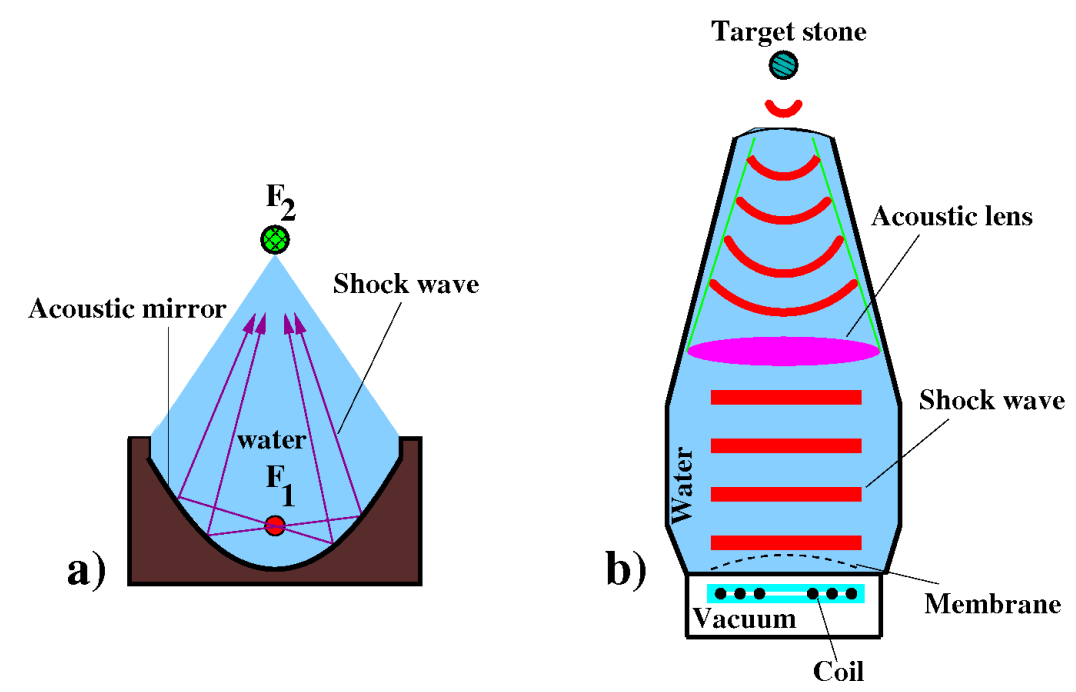

Figure 4.1: Schematic layout of electrohydraulic (a) and electromagnetic lithotripters (b).

\subsubsection{Experimental method}

In order to produce focused shock waves near to the center of a water filled container, a piezoelectric shock wave generator (PiezoSon 100, FB 12 G5, Richard Wolf, Germany) is used. It has a spherical segment radiator (see Figure 4.2) with an aperture diameter of $D \approx 110 \mathrm{~mm}$ and focal length of $F \approx 80 \mathrm{~mm}$. It contains two layers of cylindrical piezo ceramic elements which are excited by a high voltage in the range from about $2.0 \mathrm{kV}$ to $5.0 \mathrm{kV}$. The delay between excitation voltage for back and front layers of the shock wave source is about $2.5 \mu \mathrm{s}$.

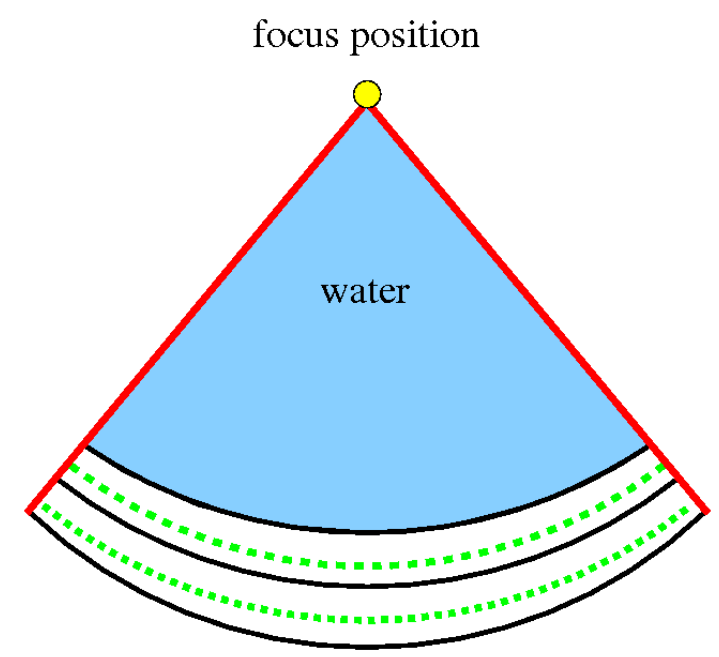

Figure 4.2: Geometry of piezoelectric shock wave generator, including two layers of piezo-ceramic elements. 
The utilization of two layers with proper delay in the voltages provides the possibility of enhancement of acoustic energy at the focus position [79, 41].

Figure 4.3 illustrates the principle function of a two piezo element layer system. If the first layer is excited at time $t$, the pressure wave reaches the interface (i.e. between transducer and load) at moment of $t^{\prime}=t+L / C$. In which $L$ being the thickness of the transducer and $C$ is the ultrasound velocity in the ceramics. Thus, the pressure wave begins to transmit into the load at this moment. The second piezo element layer is excited at the same time $\left(t^{\prime}\right)$ and then a second acoustic wave it built up by the applied voltage. In this condition, the two pressure waves strengthen each other [79].

Sferruzza et al. [79] considered the pressure waves generated by the individual shock wave generators separately, and compared it with the pressure wave produced with the sandwich model. They measured the pressure profile for the case that the first shock wave source is excited with a voltage of $8 \mathrm{kV}$ at time $t$ (when the second one is at rest). For the next step, the second shock wave generator is excited at the moment of $t^{\prime}=t+L / C$ by the voltage of $8 \mathrm{kV}$ (when the first one is at rest). They reported the pulse amplitudes of 1.3 $\mathrm{MPa}$ and 1.2 $\mathrm{MPa}$ for the first and second shock wave sources, respectively. The pressure wave amplitude for the sandwich pattern, applying the voltage of $8 \mathrm{kV}$ for each shock wave source was $2.5 \mathrm{MPa}$ i.e. the sum of the single pulse amplitudes. In this way, Sferruzza et al. concluded that the pressure waves add up in amplitude. They also reported that $L / C$ is an optimal value for the time delay, since the reduction or increasing of the delay time by $10 \%$ leads to a lower pressure pulse amplitude of $2.45 \mathrm{MPa}$.

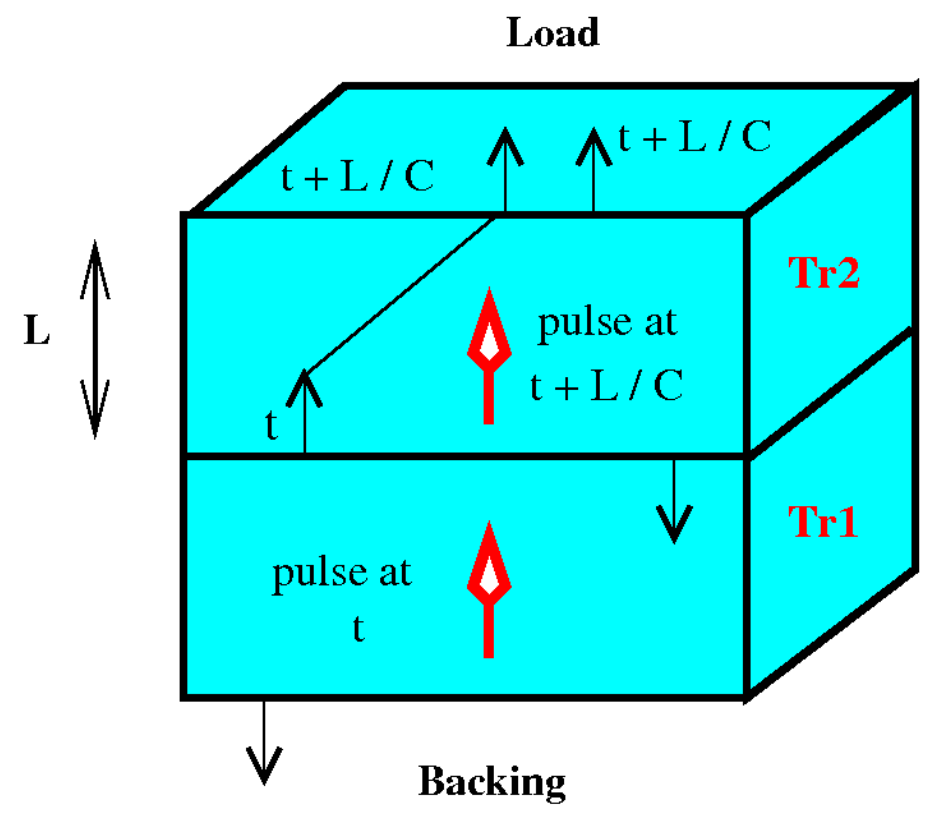

Figure 4.3: Principle function of using two layers of piezo elements. 
The PiezoSon shock wave generator offers the possibility to be used at 20 intensity levels. Each intensity level $(I)$ is related to a pair of maximum excitation voltages for back and front layers of the piezo elements $V_{b}$ and $V_{f}$. Measured excitation voltage profiles for both layers, are illustrated in Figure 4.4. The rise time ranges from about 0.6 to about $0.8 \mu \mathrm{s}$.
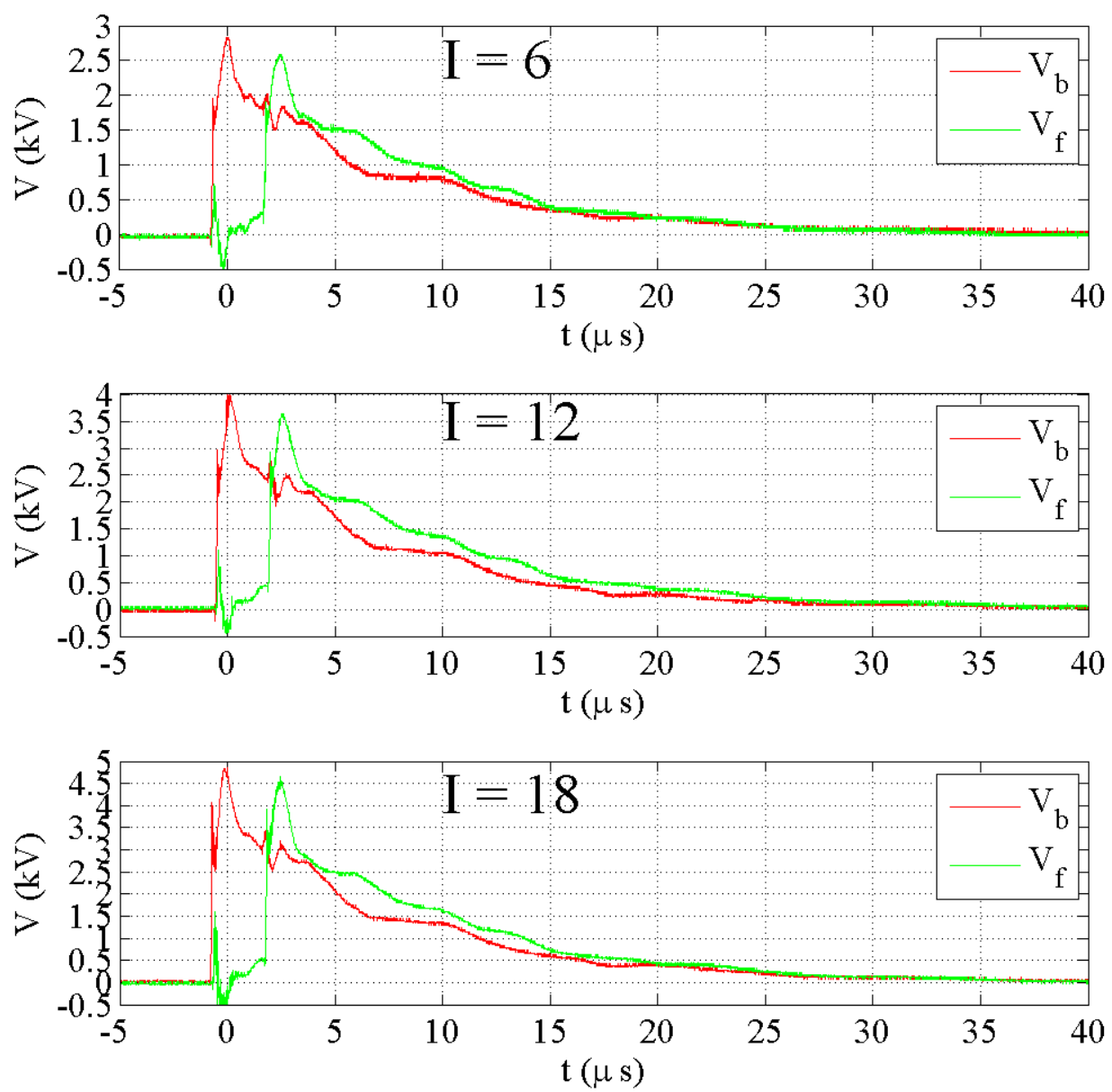

Figure 4.4: Excitation voltage of back and front layers of piezo-ceramic elements $\left(V_{b}\right.$ and $\left.V_{f}\right)$ for intensity levels $I=6,12$ and 18 of the shock wave source. For each intensity level $I$, the signals are averaged over 25 trials. Delay time between excitation voltages $V_{b}$ and $V_{f}$ is $\approx 2.5 \mu \mathrm{s}$. The peak shock pressures for different intensity levels of the shock wave source $(I)$ measured at the distance of $d \approx 1.8 \mathrm{~mm}$ above the shock focus are given in Table 5.1 . 


\subsection{Focusing of the shock waves in a cuvette}

As depicted in Figure 4.5, the spherical segment of the radiator is attached to the bottom of a water filled container with the dimensions of $16 \mathrm{~cm} \times 16 \mathrm{~cm}$ $\times 12 \mathrm{~cm}$, in order to focus the shock waves at a point on its symmetry axis.

The velocity of liquid particles at the axis of symmetry of the radiator has no component perpendicular to it [76]. The amplitude of pressure at the focus is limited due to the diffraction laws and irregular specifications of shock wave propagation (Mach reflection as described in section 3.1.2) [22, 76].

As illustrated in Figure 4.5, after excitation of the spherical segment by a high voltage, a compression wave front begins to propagate into the liquid. It is being focused at the position $C$ shown in this Figure. Two tension wave fronts originate from the opposite edges (edge rarefaction waves, ERW) [76] and intersect producing a cavitation zone. The focus of the negative phase ( $R$ in Figure 4.5) is below the focus of compression phase $C$ in this Figure, and also below the geometric focus, due to nonlinear effects [76, 23]. At the focus position, the wave front is nearly planar [42] which lets us assume a plane incident shock for the study of shock wave - bubble interaction, when a laser induced single cavitation bubble is located at the shock focus region.

The spherical geometry design of the radiator provides the opportunity of focusing the acoustic pulses without using a lens. The problem with using an acoustic lens is the fact that the lens material thickness increases in response to a rise in temperature, which modulates the focus location [3]. Another drawback is the disturbed pressure field due to reflection from lens surfaces. The reflected acoustic waves can interfere and produce a complex shape of the wave field [76]. Triboluminescence of the lens could be also another disadvantage [76]. The focusing piezoelectric shock wave source used in this work can overcome these drawbacks.

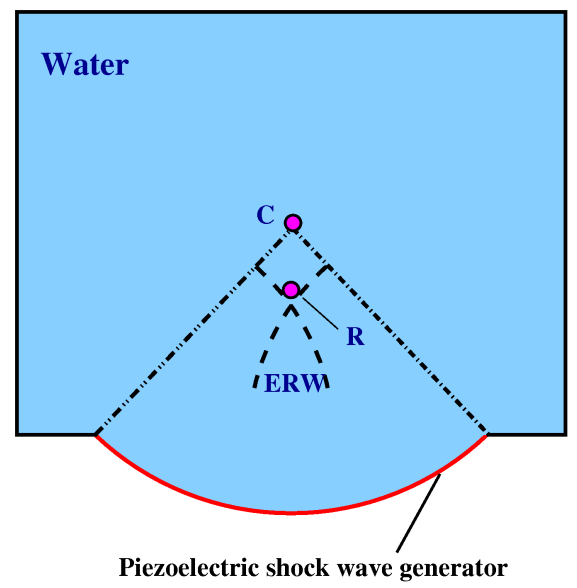

Figure 4.5: Schematic picture of the propagation of a lithotripter shock wave (LSW) in a water filled container, used for study of shock wave bubble interaction. 


\subsection{Measurement of the lithotripter shock wave (LSW) profile}

When a shock wave propagates in a medium, its density and pressure are altered. As the refractive index of the medium is a function of pressure (or density), a change in refractive index is expected in response to the shock wave passage.

Measurement of the pressure profile is performed using a fiber optic probe hydrophone (FOPH300), developed by Staudenraus and Eisenmenger at University of Stuttgart, Germany [82]. Figure 4.6 represents a schematic view of the function of the fiber optic probe hydrophone. In this device, a laser diode with an average power of about $200 \mathrm{~mW}$ and wavelength of $\lambda=810 \mathrm{~nm}$ is coupled into a glass fiber, which radiates the light into the water. Because of the difference in refractive index of water and glass, some fraction of the incident laser light is reflected and measured by a fast semiconductor photodetector (bandwidth of $30 \mathrm{MHz}$ ). This reflected light is modulated in response to the applied shock, because the refractive index of water depends on its density, thus pressure. The higher the shock wave pressure amplitude, the lower the amount of reflected laser light, because the difference between the refractive index of glass and water decreases when increasing the shock energy [85].

The photodetector signal is read by a fast oscilloscope (Infinium, $1.5 \mathrm{GHz}$, $8 \mathrm{GS} / \mathrm{s})$. The high spatial resolution of the FOPH, which is limited by the size of the fiber tip $(\approx 100 \mu \mathrm{m})$, and also the high temporal resolution of below 10 ns [85], allow a more precise measurement of the shock wave profile compared to the hydrophones based on the piezoelectric effect. The electronically limited rise time is between 5 and $10 \mathrm{~ns}$ [85]. Nevertheless, the low sensitivity (2 $\mathrm{mV} / \mathrm{MPa}$ ) could be mentioned as a disadvantage of this type of FOPH [85]. In order to increase the signal to noise ratio several recordings have to be averaged.

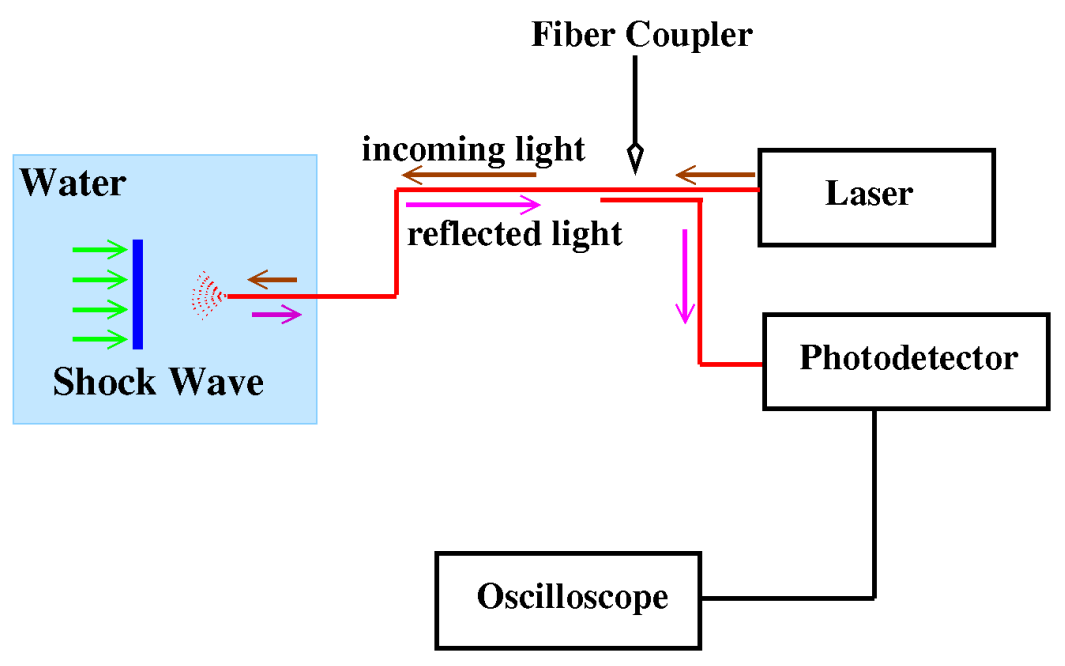

Figure 4.6: Schematic image of the function of fiber optic probe hydrophone (FOPH300) [85]. 
The absolute value for the pressure is derived by calibration and deconvolution of the pressure signal by the response function of the FOPH.

\subsection{Calibration}

The self-calibration property of the FOPH allows it to be calibrated without using other standard sources [100]. Let us define $R_{0}$ and $U_{0}$ as the reflection coefficient and photodetector DC voltage related to the undisturbed medium, respectively. The change in reflection coefficient of the medium in response to the applied shock wave $\Delta R(p)$ corresponds to the photodetector voltage change $\Delta U(p)$. Therefore, the parameters $R$ and $U$ are pressure $(p)$ dependent. Considering the stray light which is scattered at the optical fiber tip and defining parameter $\alpha$ as the internal stray light coefficient, we get [85]:

$$
\frac{\Delta R(p)}{R_{0}}=(1+\alpha)\left(\frac{\Delta U(p)}{U_{0}}\right)
$$

The relation between the pressure dependent refractive index of the medium $n(p)$ and variation of the reflection coefficient $R(p)$, can be derived by Fresnel's formula with the assumption of vertical light incidence [85]:

$$
\Delta R(p)=R(p)-R_{0}=\frac{\left(n(p)-n_{\text {glass }}\right)^{2}}{\left(n(p)+n_{\text {glass }}\right)^{2}}-\frac{\left(n_{0}-n_{\text {glass }}\right)^{2}}{\left(n_{0}+n_{\text {glass }}\right)^{2}},
$$

where $n_{\text {glass }}=1.483$ is the refractive index of the fiber optic core, $n_{0}=1.329$ is the refractive index for water at atmospheric pressure, at temperature of $20^{\circ}$ $\mathrm{C}$ and wavelength of $\lambda=810 \mathrm{~nm}$.

As the shock front propagates in the liquid, the refractive index of the medium is modulated due to the variation in the pressure and density. By using the equation of state (3.1) which holds in water for the pressures up to $1 \mathrm{GPa}([82])$ and the Gladstone-Dale equation considering the dependency of the refractive index of the medium on the mass density ([85]) yields:

$$
n(p)=1+\left(n_{0}-1\right)\left[1+\frac{\left(p-p_{0}\right)}{\left(p_{0}+B\right)}\right]^{\frac{1}{\gamma}} .
$$

The parameters $B=295.5 \mathrm{MPa}$ and $\gamma=7.44$ are valid for water at room temperature. This dependency can be used in order to measure the shock strength by optical means.

In order to derive a relation between the change in the measured photodetector voltage $\Delta U$ and shock pressure $p$, we insert equations (4.2) and (4.3) into (4.1). Considering the parameters defined in table 4.1, it reads [85]:

$$
\frac{\Delta p}{M P a}=a\left[\frac{b+c \frac{\Delta U}{U_{0}}(1+\alpha)-4 \sqrt{d\left(\frac{\Delta U}{U_{0}}(1+\alpha)+1\right)}}{e-f\left(\frac{\Delta U}{U_{0}}\right)(1+\alpha)}\right]^{g}-h
$$


Table 4.1: Constant parameters of equation 4.4.

\begin{tabular}{cc|cc}
\hline \hline $\mathrm{a}$ & 1.7023 & $\mathrm{e}$ & 0.8545 \\
$\mathrm{~b}$ & 2.567 & $\mathrm{f}$ & 0.00263 \\
$\mathrm{c}$ & 0.0398 & $\mathrm{~g}$ & 7.44 \\
$\mathrm{~d}$ & 0.0460 & $\mathrm{~h}$ & 295.5 \\
\hline
\end{tabular}

Equation (4.4) includes nonlinearity which is more pronounced for pressures higher than $+30 \mathrm{MPa}$ and greater than $-10 \mathrm{MPa}$ [85]. It holds for the water temperature of $20^{\circ} \mathrm{C}$. Taking into account the compression of the fiber tip itself in response to the applied shock and also the effect of change in water temperature (in $\mathrm{K}$ ), one obtains [85]:

$$
\Delta P(T)=\Delta p .1 .036 .(1+0.003 / K \cdot(T-293 K)),
$$

where the quantity $\Delta P(T)$ is the change in the pressure at temperature $T$.

\subsection{Deconvolution}

Due to the small size of the hydrophone tip $(\approx 100 \mu \mathrm{m})$ compared to the focal size of the shock wave, it could be assumed that the incident shock at the fiber tip is a plane wave. The fiber tip can be regarded as a rigid boundary because of the large difference between acoustic impedance of the fiber material (Silica) and that of water. Diffraction of acoustic wave by the fiber optic end is studied in [100].

In order to obtain the correct value for the shock pressure, the response of the FOPH to the shock front has to be examined. Acoustic reflection of the shock front at the fiber tip is accompanied by an increase in the pressure by a factor of two at this location, due to the characteristics of rigid reflection. This fact leads to an overshoot in the step response of the hydrophone. The response of the FOPH to both a plane pressure step and a self-focusing shock wave includes an overshoot of $80 \mathrm{~ns}$ half width at the leading edge [100], as illustrated in Figure 4.7 [85]. In this work, a code developed by the FOPH producer is used for deconvolution, in which the effect of overshoot in the response of the fiber optic hydrophone is corrected. 


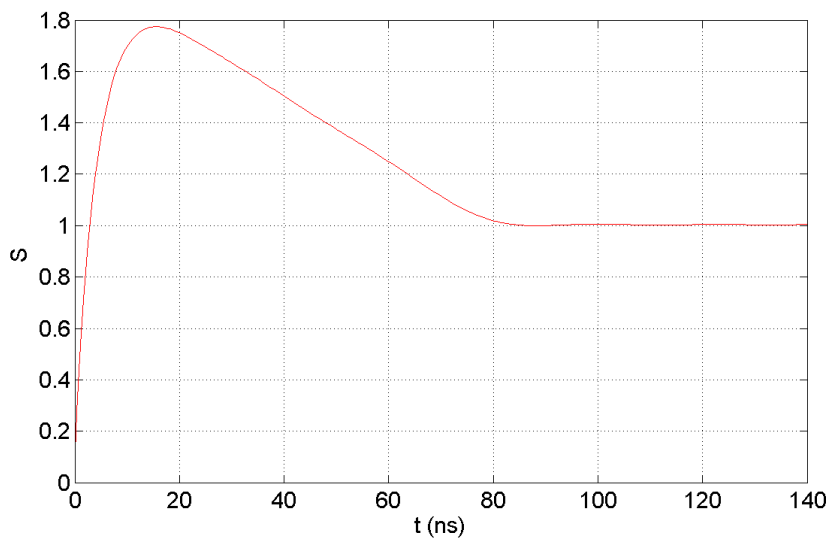

Figure 4.7: Simulated step response (S) of fiber optic probe hydrophone at the photodetector bandwidth of $40 \mathrm{MHz}$ [85].

\subsection{Shock wave pressure profile}

Figure 4.8 depicts the lithotripter shock wave (LSW) profile for three different intensity levels of the shock wave source. The measurement is performed at a distance of $d \approx 1.8 \mathrm{~mm}$ above the shock wave focus position, which is the same distance as in the shock pressure measurement performed in chapter 5 . In order to increase the signal to noise ratio, for each intensity level (pairs of shock wave generator peak voltages $V_{b}$ and $V_{f}$ ), 20 signals are averaged. The compression phase is followed by a longer tension tail as displayed in Figure 4.8. The pulse duration of the positive part of the shock wave is $\approx 1 \mu \mathrm{s}$, while the duration of the tension phase is $\approx 3 \mu \mathrm{s}$.

The peak pressure pulse amplitude (the compression part) ranges from $P_{+}=$ $24.4 \pm 1.1 \mathrm{MPa}$ to $P_{+}=108.1 \pm 2.5 \mathrm{MPa}$, for maximum voltages of the shock wave source (back layer) between $V_{b}=2.53 \pm 0.020 \mathrm{kV}$ and $V_{b}=$ $4.99 \pm 0.117 \mathrm{kV}$, respectively. The negative tail of the shock front has an amplitude ranging from $P_{-} \approx-6.9 \mathrm{MPa}\left(\right.$ for $V_{b} \approx 2.5 \mathrm{kV}$ ) to $P_{-} \approx-13.6 \mathrm{MPa}$ (for $V_{b} \approx 4.6 \mathrm{kV}$ ). These shock waves can be used to study the interaction between shock wave and laser-generated single cavitation bubbles. The compression wave is strong enough to disturb the bubbles and force them to collapse more violently. Nevertheless, the negative part of the shock wave is able to produce secondary cavitation, which can modulate the dynamics of laser-generated single bubbles. The interaction of a shock wave with single cavitation bubbles is discussed in chapters 5 and 6.

The shock front velocity is derived for pulse amplitudes of $P=30,62$ and $108 \mathrm{MPa}$, respectively. Measurement is performed considering the difference in the shock arrival times at the positions of $z_{1} \approx 0.8 \mathrm{~mm}$ and $z_{2} \approx 2.9 \mathrm{~mm}$ above the shock wave focus location. The shock front velocities are $U_{s h} \approx 1623.6 \mathrm{~m} / \mathrm{s}$, $U_{s h} \approx 1639.7 \mathrm{~m} / \mathrm{s}$ and $U_{s h} \approx 1654.8 \mathrm{~m} / \mathrm{s}$ for the mentioned pulse amplitudes. 
It can be seen that the shock propagation becomes faster by increasing the pulse amplitude. This is due to nonlinear effects described in chapter 3 .

The pressure pulse amplitude measured at the distance of $d \approx 1.8 \mathrm{~mm}$ above the shock focus is plotted for application of different values of peak voltage $V_{b}$, in Figure 4.9. As the maximum voltages $V_{b}$ and $V_{f}$ are dependent on each other for each intensity level of the shock wave source, just the results related to voltage $V_{b}$ are presented. A quadratic fit to the experimental data is applied with the following relation:

$$
P=6.3 V_{b}^{2}-13.0 V_{b}+15.6
$$

where $P$ is in $\mathrm{MPa}$ and $V_{b}$ being in $\mathrm{kV}$.
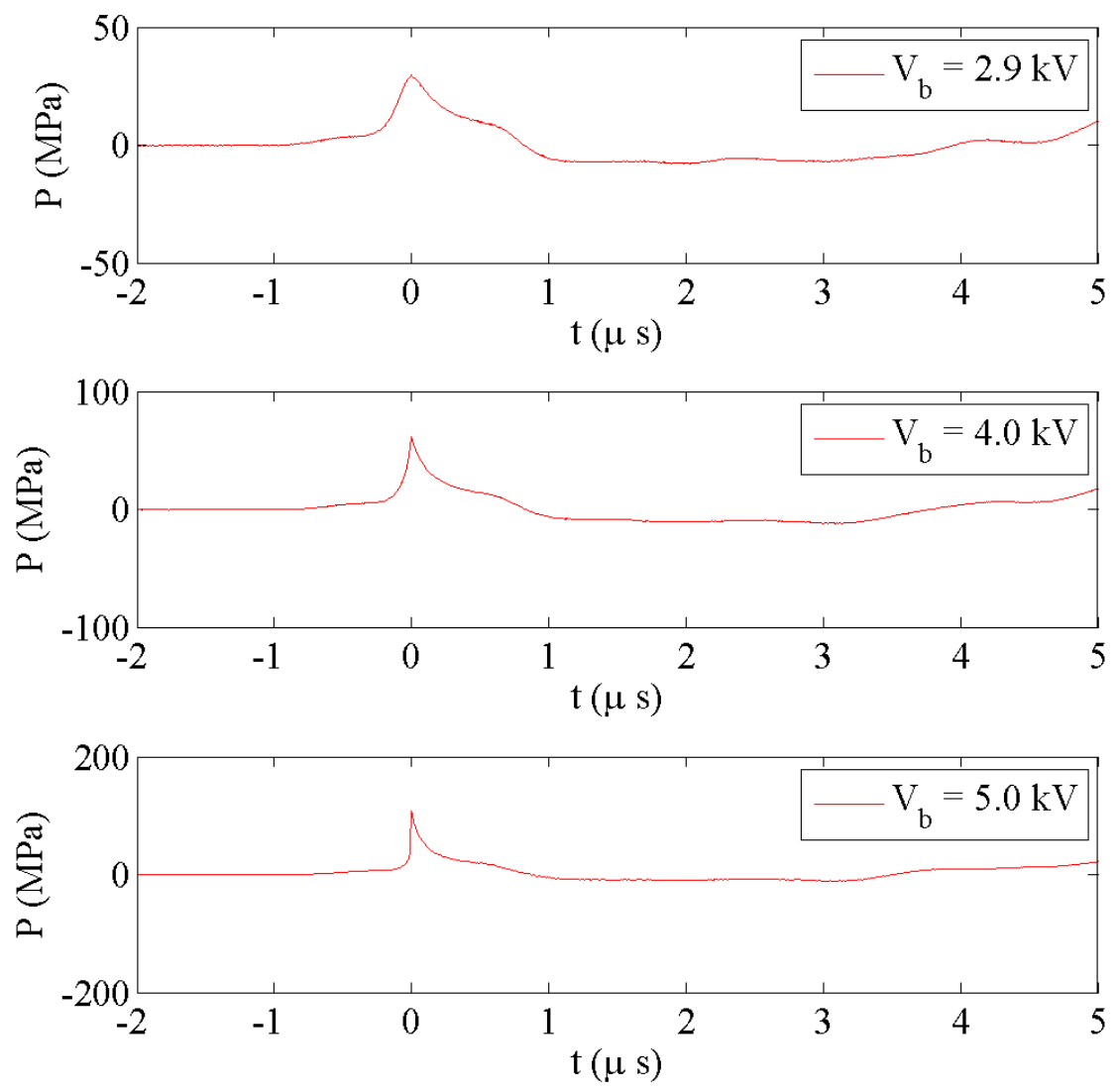

Figure 4.8: Pressure wave profile of the lithotripter shock wave (LSW) in the free field for three intensity levels of the shock wave source $(I=6$ $\left(V_{b}=2.9 \mathrm{kV}\right), I=12\left(V_{b}=4.0 \mathrm{kV}\right)$ and $I=18\left(V_{b}=5.0 \mathrm{kV}\right)$. For each intensity level, the signal is averaged over 20 measurements. Full width at half maximum (FWHM) of the compression part is decreasing for stronger shock waves. The pulse duration of the tension phase is relatively longer than that of the positive part. 


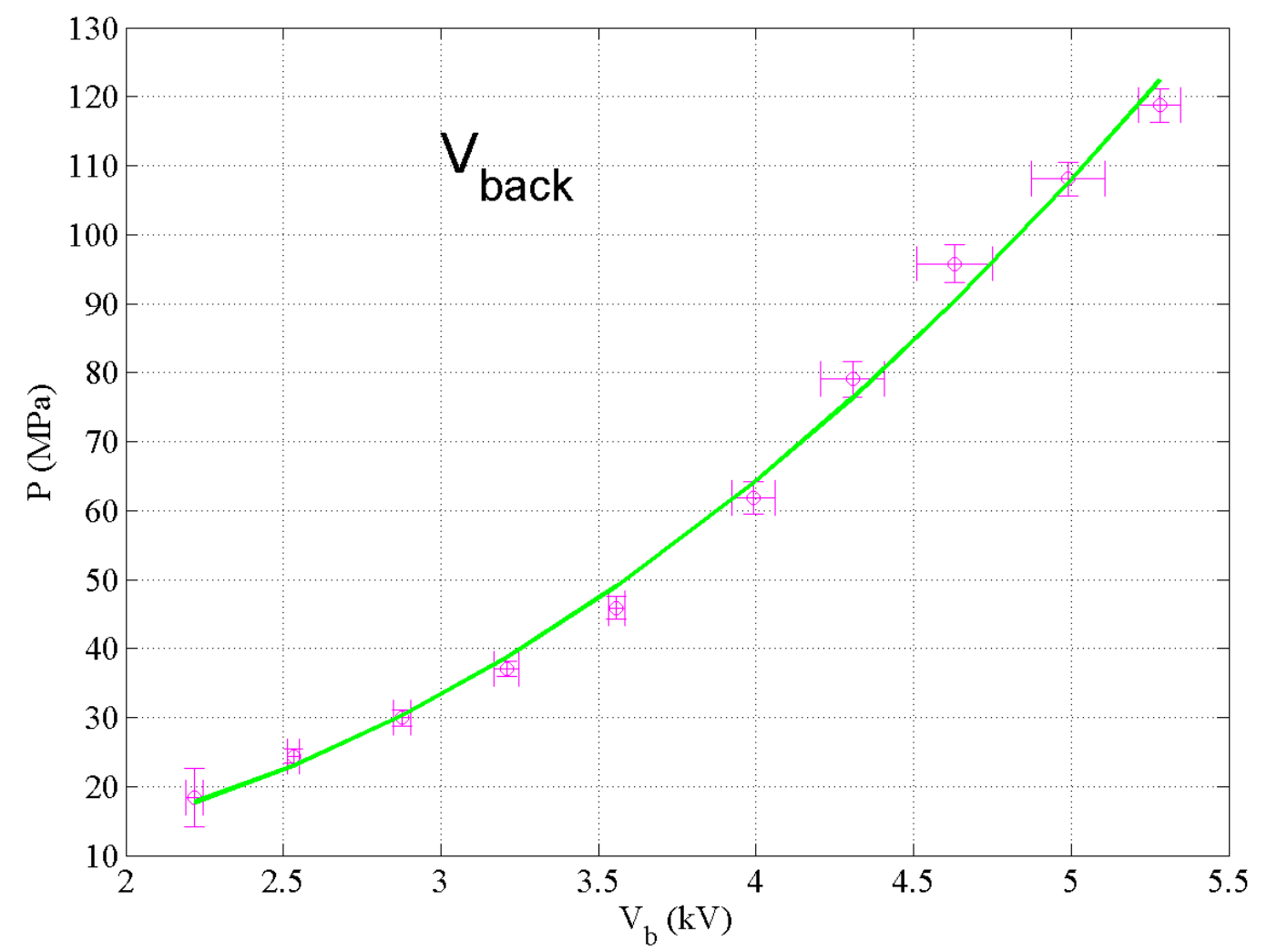

Figure 4.9: Lithotripter shock wave pulse amplitude $P$ as a function of peak excitation voltage of the shock wave source (back layer). Error bars are related to standard deviation of the experimental data. The solid line represents a quadratic fit to the measured values. The measurement is performed at the distance of $d \approx 1.8 \mathrm{~mm}$ above the position of the shock wave focus in the free field. 
In order to investigate the temporal profile of the lithotripter pressure waves used in the study of shock wave - bubble interaction, the full width at half maximum $(\tau)$ of the pressure wave profiles is considered. It seems that $\tau$ is decreasing with the shock wave pulse amplitude. As shown in Figure 4.10, it drops from $\tau=480 \mathrm{~ns}$ to $\tau=90 \mathrm{~ns}$ by increasing the pulse amplitude from $P=18.4 \pm 4.3 \mathrm{MPa}$ to $P=108.1 \pm 2.5 \mathrm{MPa}$.

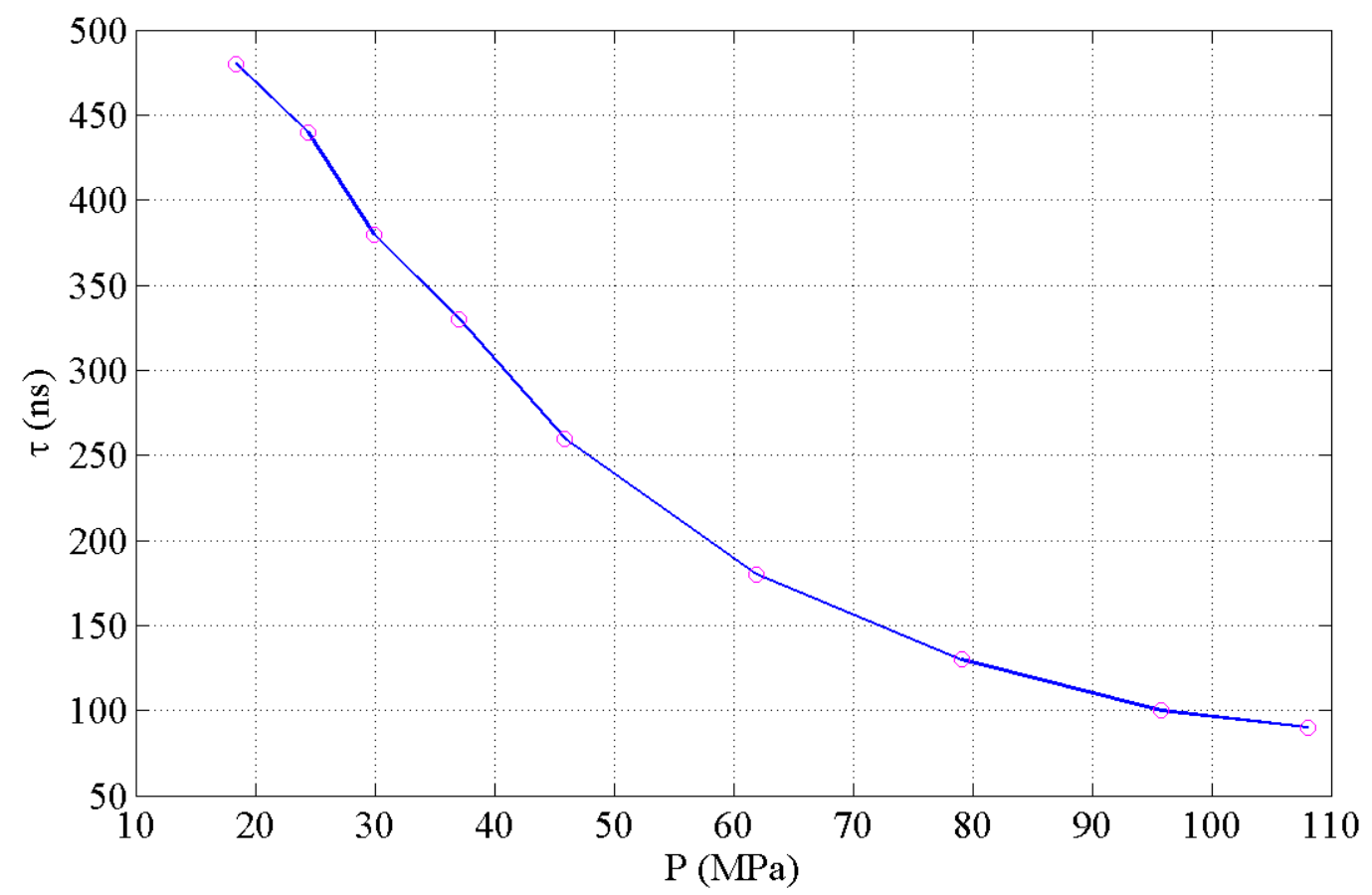

Figure 4.10: Full width at half maximum (FWHM) $\tau$ of the shock wave profile for different pulse amplitudes $(P)$.

\subsection{Bubble formation}

Tensile strength of water depends on its impurities and gas content [84, 73]. The value of about -5 bar is suggested by Crum [24] for normal situations. Then, cavitation bubbles are initiated in the case the tensile strength of water being surpassed. The bubbles can expand in the negative pressure phase of the shock wave profile by about three orders of magnitude and last for more than $200 \mu \mathrm{s}$ [73]. In this period, the bubbles oscillate in response to the static pressure of water, and the gas diffusion into them during the oscillation is more than the outward gas diffusion [73]. This phenomenon is called "Rectified diffusion" [25]. When the bubble contracts, the inner pressure rises and the gas diffuses from the cavity, and when the bubble expands, the pressure in the bubble drops and the gas diffuses inward. As the diffusion rate is increasing with area, more gas will enter to the bubble during its expansion compared 
to the gas which leaves the cavity during its contraction. This is an area effect. Another feature which enhances the rectified diffusion, is the shell effect. During the bubble contraction, an asymptotic spherical shell surrounding the bubble expands and the gas concentration gradient near to the bubble wall lowers. Then, for a short moment the gas diffusion rate out of the bubble is larger than in the case that the cavity is at equilibrium radius. When the bubble expands, the shell contracts and the gas concentration gradient near to the bubble wall rises, which leads to the fact that the gas diffusion rate into the bubble being higher than the average for a larger time span [25]. The amplitude of the tension phase of the applied shock wave (i.e. from $P_{-} \approx-6.9 \mathrm{MPa}$ to $\left.P_{-} \approx-13.6 \mathrm{MPa}\right)$ is above the mentioned tensile strength of water. Therefore, some small cavitation bubbles are expected to be visible in the sound field.

Figure 4.11 illustrates the standard deviation of the measurement of the pressure wave profiles for two positive pulse amplitudes $P=61.9 \pm 2.4 \mathrm{MPa}$ and $P=29.9 \pm 1.3 \mathrm{MPa}$. The measurement is performed in deionized, partially degassed and filtered water as well as tap water. The signal appears more noisy in the case of using tap water. This indicates the effect of the bubbles which are initiated in the sound field. The standard deviation of the pressure measurement for the case of using deionized, filtered and partially degassed water is nearly constant while there are some fluctuations in the case of application of tap water.
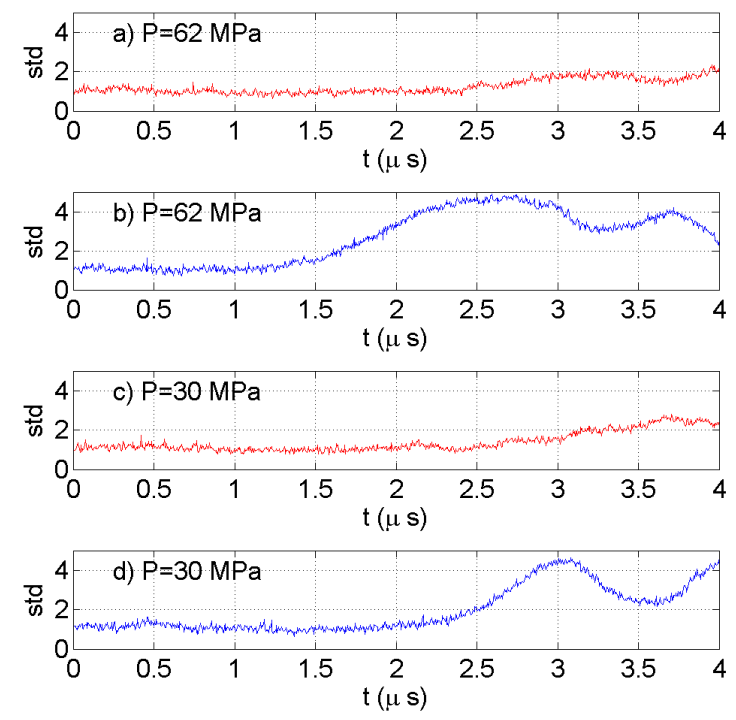

Figure 4.11: Standard deviation of the pressure wave measurement for deionized, filtered and partially degassed water (a and c) and tap water $(b$ and $d))$. 


\subsection{Shock wave propagation in water}

Shadowgraph images are recorded using a CCD camera (pco. imaging sensicam, Germany) in its single frame mode. An LED flash lamp with pulse duration of $\tau \approx 8 \mathrm{~ns}(\mathrm{FWHM})$ is applied for back illumination. The short duration of the flash lamp pulses provides the possibility of capturing the fast traveling shock waves in a medium like water. Although the exposure time of the camera is much longer than this time interval, the effective exposure time is limited to the pulse duration of the flash lamp.

In order to illustrate the shock wave passage in water, shadowgraph pictures are shown in Figure 4.12. The Mach stem (see chapter 3) is visible in the frames where the shock wave arrives in the focus position. Some small bubbles are seen at the bottom of the lower frames (about $1.2 \mu \mathrm{s}$ after passage of the shock wave from its focus position). They begin to growth and their population is increasing by time. Radii of these cavitation bubbles range from $R \approx 20 \mu \mathrm{m}$ to $\approx 240 \mu \mathrm{m}$.

The acoustic field at the moment of $t \approx 15 \mu$ s after the passage of the lithotripter shock wave (LSW) from the focus position is shown in Figures 4.13 and 4.14. These images are related to application of deionized, filtered, partially degassed water and also tap water, respectively. The effect of water impurity and gas content can be seen by comparing these two Figures. The population of cavitation bubbles is enhanced by using tap water. The bubbles in this field have maximum radius of $R \approx 400 \mu \mathrm{m}$ at the moment of recording of the corresponding images. The pressure pulse amplitude at distance of $d \approx 1.8 \mathrm{~mm}$ above the focus location is $P=108.1 \pm 2.5 \mathrm{MPa}$.

Figure 4.15 displays bubble clouds in the acoustic field at the moment of $t \approx 9 \mu \mathrm{s}$ after the passage of the shock wave at its focus position. It can be seen that the spherical collapse of individual cavitation bubbles enhances their neighboring bubble implosions. Also some shock induced liquid jets are visible. The maximum bubble radius in this case is $\approx 310 \mu \mathrm{m}$. This image shows bubble - bubble interaction as well as shock wave - bubble interaction. 


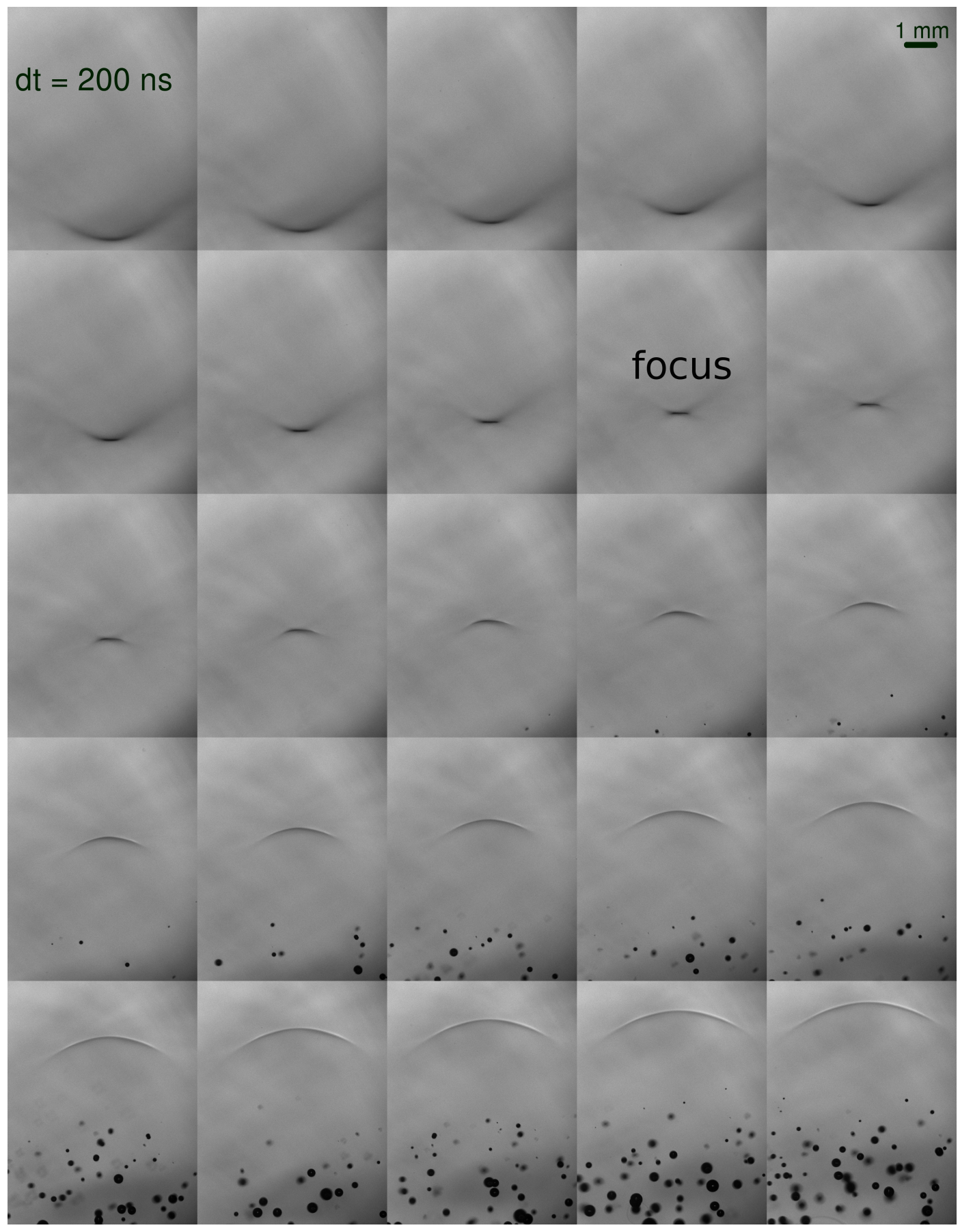

Figure 4.12: Image sequence of the shock wave propagation in water. The Mach stem is visible in the frames that the shock front is nearly focused. After passage of the the shock wave secondary cavitation takes place. 


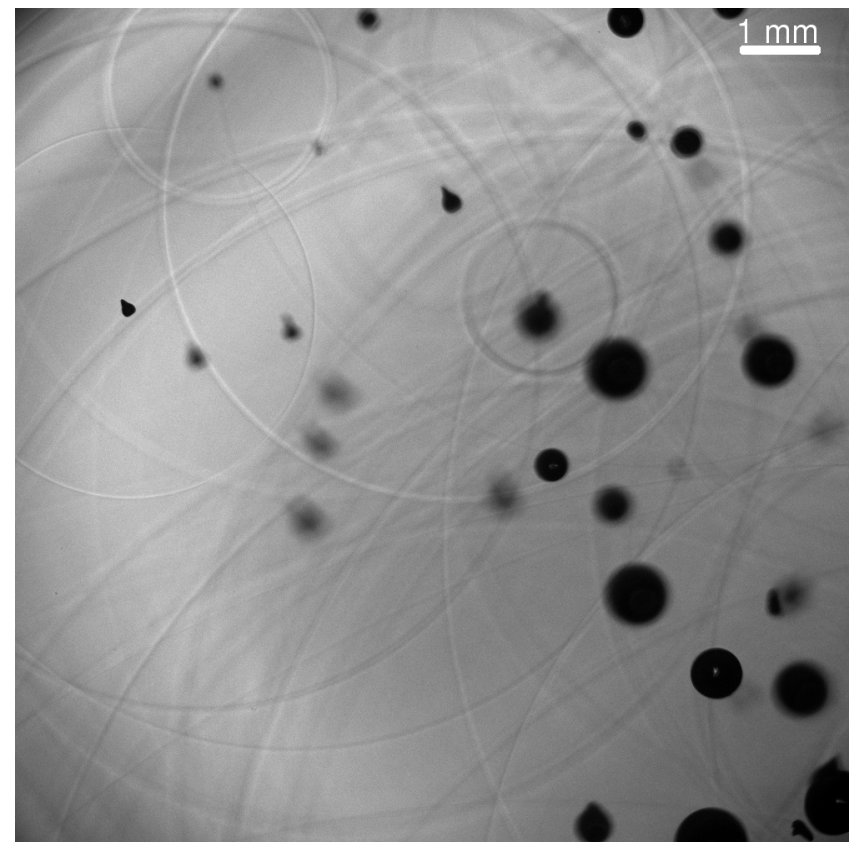

Figure 4.13: Acoustic field at the moment $t \approx 15 \mu$ s after the passage of the shock wave when using deionized, partially degassed and filtered water. The pulse amplitude is $P=108 \mathrm{MPa}$.

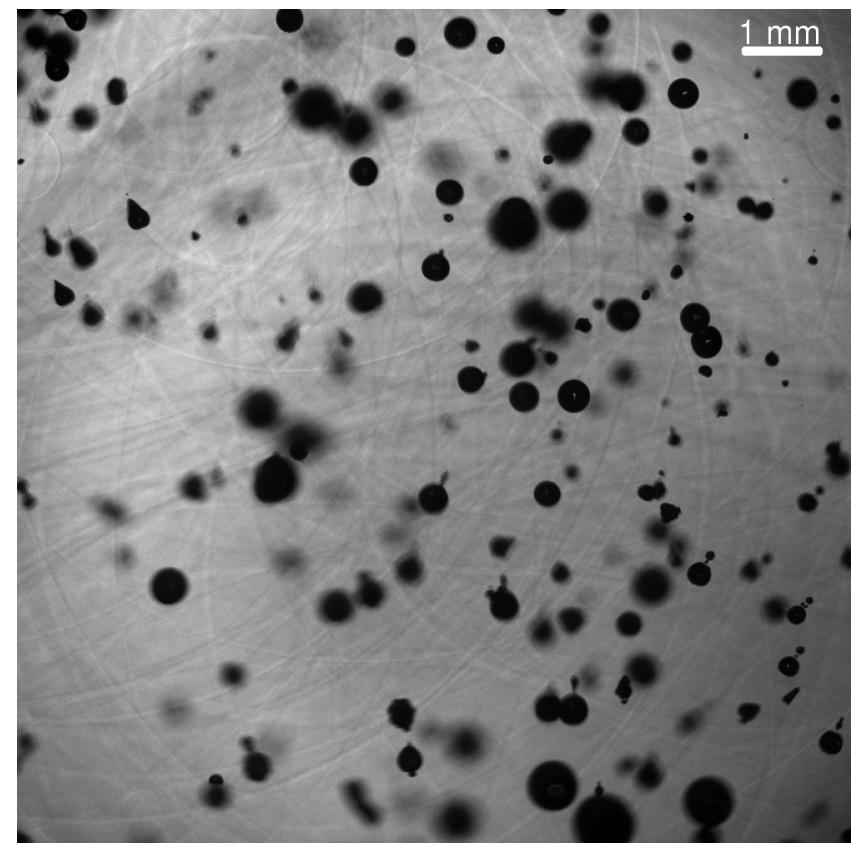

Figure 4.14: Acoustic field at time $t \approx 15 \mu$ s after the passage of the shock wave, by application of tap water. The bubble population is enhanced and therefore, the acoustics field appears to be more noisy compared to Figure 4.13. The pulse amplitude is $P=$ $108 \mathrm{MPa}$. The radius of the initiated cavitation bubbles is as large as $R \approx 400 \mu \mathrm{m}$. 


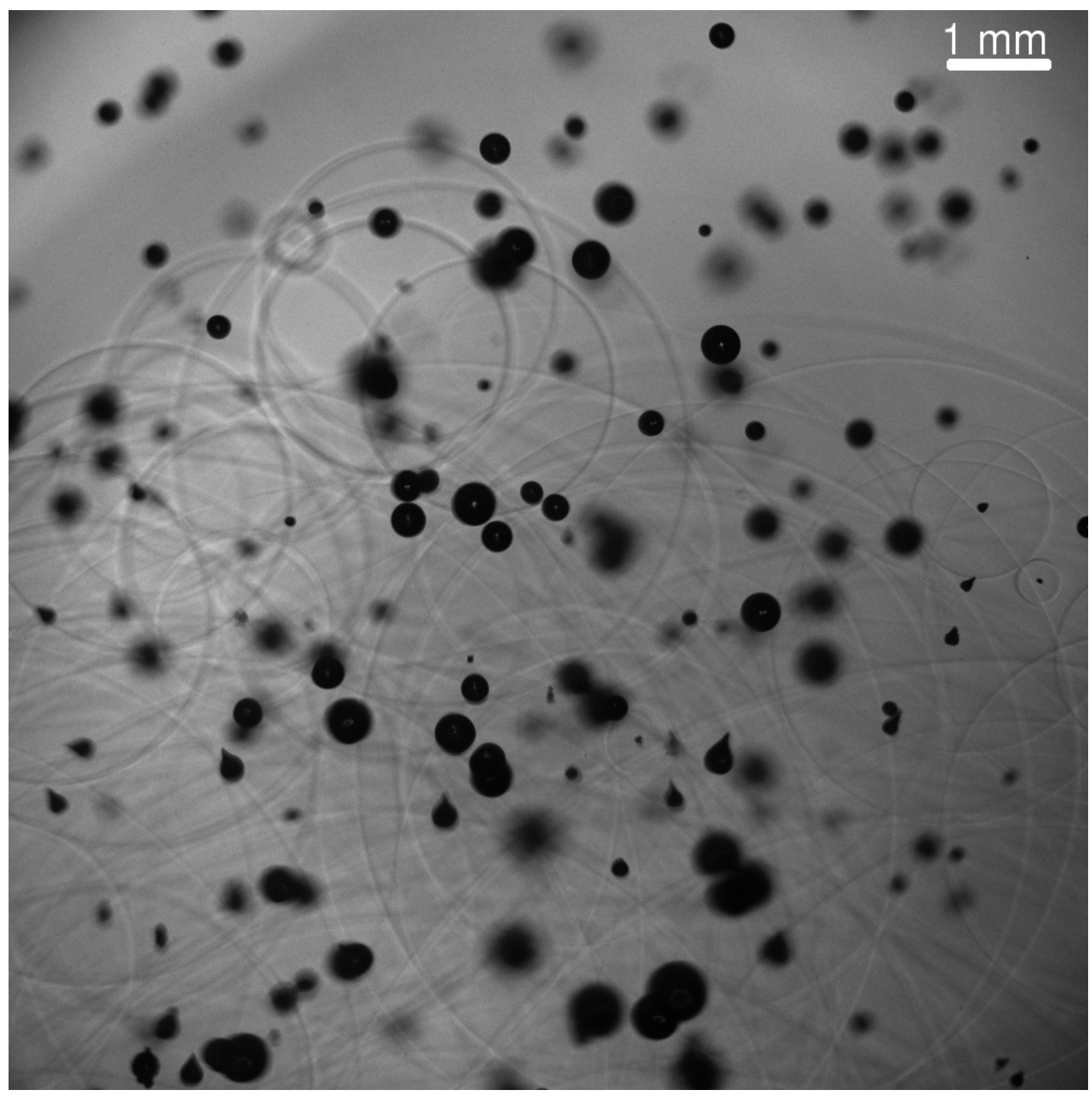

Figure 4.15: Acoustic field at moment $t \approx 9 \mu$ s after the passage of the shock wave, using tap water. The pulse amplitude is $P=108 \mathrm{MPa}$. The maximum radius of the bubbles is $R \approx 310 \mu \mathrm{m}$ at this moment. 


\section{Chapter 5}

\section{Interaction between lithotripter shock waves and laser-generated single cavitation bubbles}

In this work, the behavior of laser-induced single cavitation bubbles in response to impact of a lithotripter shock wave is investigated by means of high-speed photography and acoustic field measurements. The effect of variation of the shock wave energy is considered. A shock wave impinges on the bubbles at different moments of their oscillation phase, which provides the possibility of studying the influence of the initial bubble oscillation phase on the interaction. Enhancement of the pressure wave emitted from bubble forced implosion as well as reduction of the collapse time relative to the inertial cavitation bubble oscillation, have been explored. Shock wave-induced liquid jetting has been considered for different shock wave energies. The effect of the presence of a solid interface nearby to the cavity parallel to the direction of shock wave propagation is studied in the next chapter using different wall distances.

\subsection{Method}

In order to study shock wave - bubble interaction, we need to have precise temporal and spatial information about cavitation bubbles. The single cavitation bubbles have to be well localized and the bubble generation process to be synchronized with the rest part of the experiment. For this purpose, a Q-switched laser source (Brio, Quantel) with a second harmonic generation (SHG) module at wavelength of $\lambda=532 \mathrm{~nm}$ and with pulse duration of $\approx 4 \mathrm{~ns}$ is used. The laser beam has a diameter of $\approx 4 \mathrm{~mm}$. It is widened by an $8 \times$ beam expander in order to reduce the spot size and also increase the numerical aperture of the optical system. An aberration minimized lens system with focal length of inner lens as $\approx 50 \mathrm{~mm}$ in water has been applied (numerical aperture $(\mathrm{NA})$ is $\approx 0.3$ ). 
The quality of the laser focus plays an important role in reproducibility of the induced cavitation bubbles in different trials. It has also a major effect on the sphericity of the generated bubbles. The shock focus position is located on the center axis of a water filled tank with the dimensions of $(16 \mathrm{~cm} \times 16 \mathrm{~cm}$ $\times 12 \mathrm{~cm}$ ) right at the location of the laser focus. The laser energy has been reduced to a few $\mathrm{mJ}$ using a combination of a $\lambda / 2$ wave plate and a cubic polarizing beam splitter. For back illumination, a bright flash lamp (Metz Mecablitz $60 \mathrm{CT}-4)$ is applied. In order to record the images, a long distance microscope (K2 Infinity) attached to a very high-speed camera (Imacon 468, DRS Hadland Ltd.) has been used. The camera consists of 8 independent intensified CCDs, and is capable of taking pictures with frame rates of up to 100 million frames/sec. Delay time between individual channels can be varied by the help of an electronic box inside the camera.

A piezoelectric shock wave generator (PiezoSon 100, FB 12 G5, Richard Wolf Germany) described in chapter 4, produces lithotripter shock wave pulses which are focused at the center of the water filled container (the place where cavitation bubbles are generated via optical breakdown). For recording of the acoustic field at top of cavitation bubbles, a fiber optic probe hydrophone (FOPH300, Univ. Stuttgart, Germany [82]) as discussed in chapter 4, has been utilized.

In order to synchronize the laser source, shock wave generator, flash lamp and camera, two delay generators (DG535, Stanford research systems and a workshop made $10 \mathrm{~Hz}$ clock with a trigger selection box electronic circuit) have been applied. Figure 5.1 shows a schematic view of the synchronization procedure. Figures 5.2 and 5.3 display schematic layouts of the experimental setup.

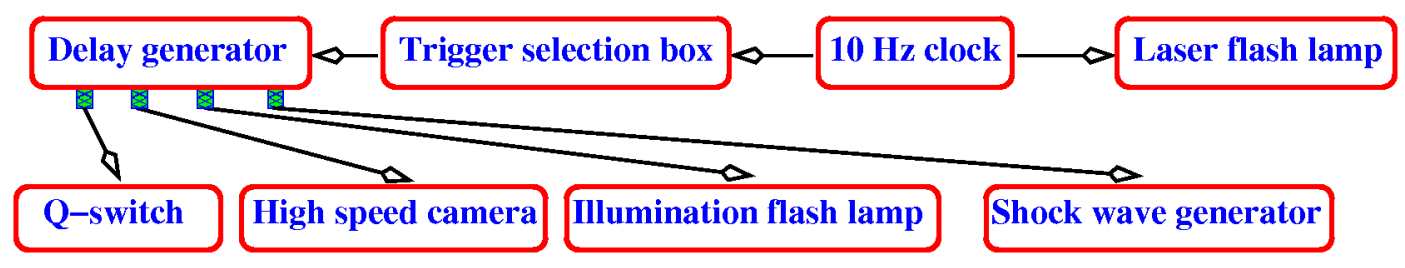

Figure 5.1: Schematic view of the synchronization procedure of the experimental setup. 


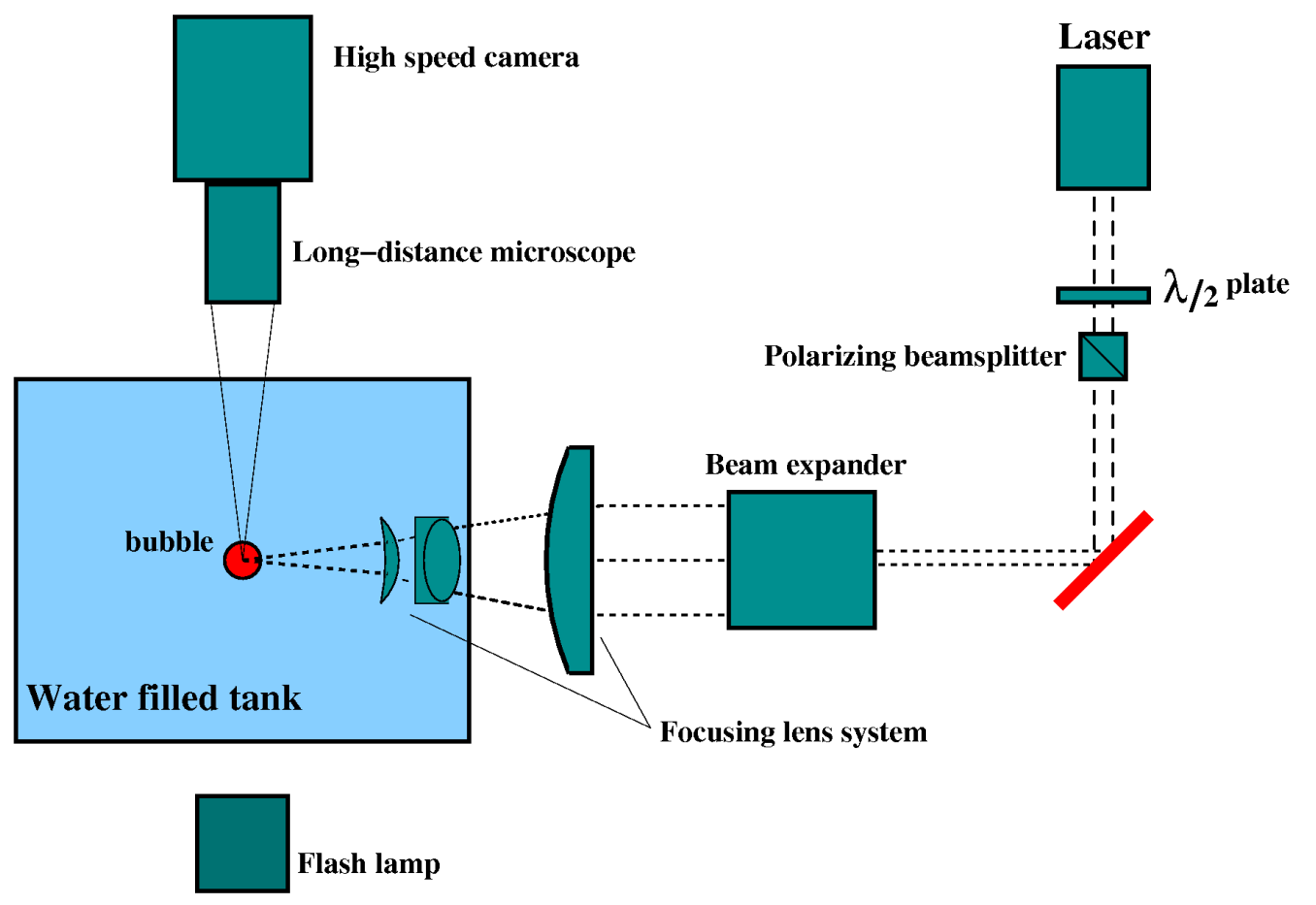

Figure 5.2: Schematic picture of the experimental setup for the study of interaction between lithotripter shock waves and laser-induced single cavitation bubbles (top view).

Fiber optic probe hydrophone

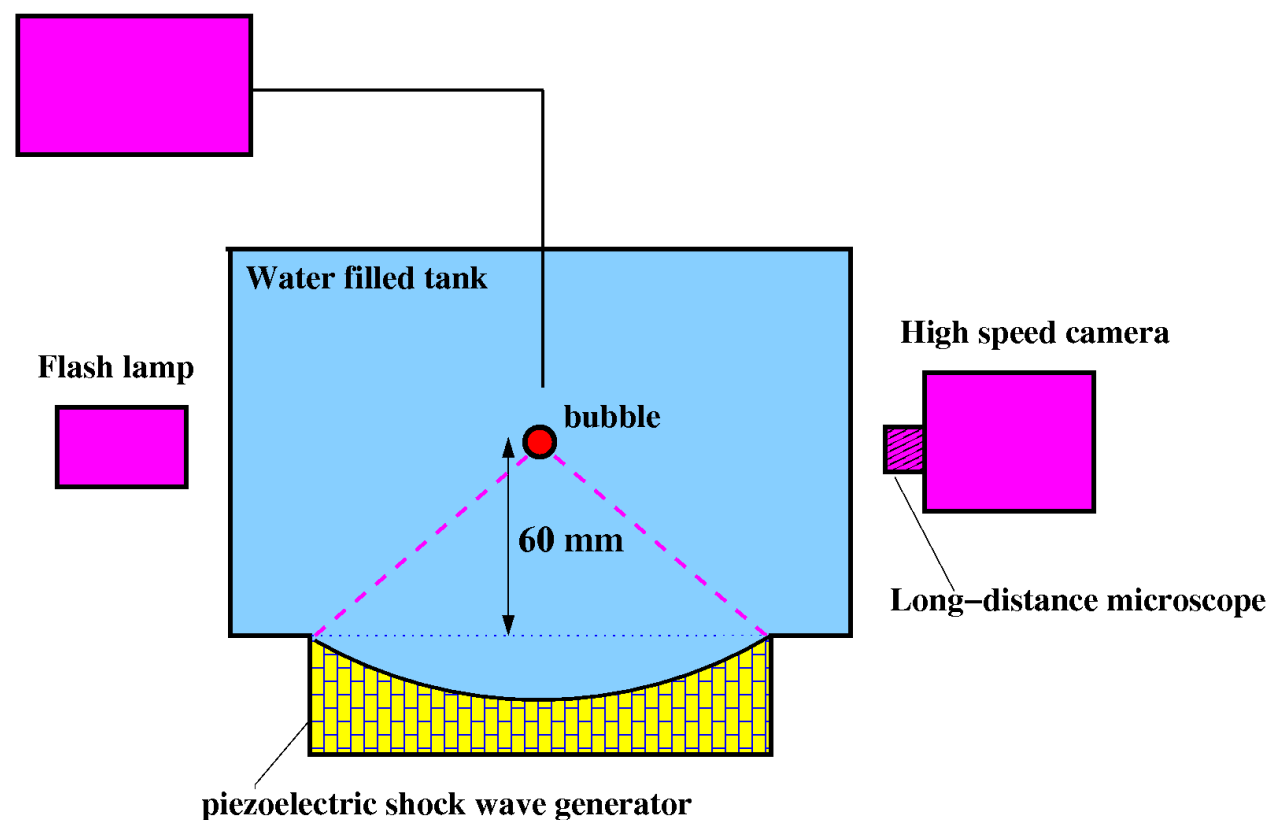

Figure 5.3: Schematic layout of the experimental setup for investigation of shock wave - bubble interaction (side view). 


\subsection{Laser generation of cavitation bubbles}

The technique of laser-induced cavitation (optical breakdown) was utilized for single cavitation bubble research by Lauterborn since 1972. He focused ruby laser pulses into liquids to generate cavitation bubbles [51]. The maximum size of cavities can be controlled by variation of laser energy. The induced cavitation bubbles are well localized, and at any specific moment, the bubble size can be determined. These properties allow investigation of shock wave bubble interaction in case that the shock wave arrives at the location where the cavitation bubbles are initiated.

When a laser beam is focused in water and the threshold irradiance for breakdown is exceeded, a plasma is produced due to nonlinear absorption [95]. The plasma formation is followed by generation of shock waves as well as cavitation in the liquid [95]. The initial shock wave has a distorted elliptical shape at the moment of $t \approx 130 \mathrm{~ns}$ after the optical breakdown [95]. For pulse duration of $6 \mathrm{~ns}$, the plasma lengths are $112 \mu \mathrm{m}$ for laser energy of $1 \mathrm{~mJ}$ and $260 \mu \mathrm{m}$ for laser energy of $10 \mathrm{~mJ}$ [66]. The temperature of the plasma for nanosecond pulses is estimated as $T \approx 10,000 \mathrm{~K}$ [99] and $T \approx 15,000 \mathrm{~K}$ [5, 99], while for picosecond pulses it is $T \approx 6,200 \mathrm{~K}$ [99].

Noack et al. [66] reported the shock peak pressure at the source as $8.4 \pm$ $1.5 \mathrm{GPa}$ and $11.8 \pm 1.6 \mathrm{GPa}$ for laser pulse duration of $6 \mathrm{~ns}$ and laser energies of $1 \mathrm{~mJ}$ and $10 \mathrm{~mJ}$, respectively. After plasma recombination, the induced cavitation bubble expands and forms a spherical cavity [2]. Because of geometrical damping and dissipation, the acoustic energy is reduced by expansion.

According to the experimental results of Fujimoto et al. [35], the initial velocity of the shock wave is approximately $2100 \mathrm{~m} / \mathrm{s}$ for a pump laser with pulse energy of $10 \mathrm{~mJ}$. This velocity is reduced to $\approx 1700 \mathrm{~m} / \mathrm{s}$ after a propagation of distance about $100 \mu \mathrm{m}$ (in $t \approx 50 \mathrm{~ns}$ ) and remains constant thereafter. This value corresponds to the velocity of sound in water which is about $1500 \mathrm{~m} / \mathrm{s}$.

Figure 5.4, illustrates a shadowgraph image of the laser-induced cavitation bubble in water. The shock wave originating from laser breakdown is nearly spherical. Interframe time is $150 \mathrm{~ns}$. A typical pressure time history of shock waves induced by optical breakdown and bubble collapse for a free cavity is shown in Figure 5.5. The measurement is performed using a fiber optic probe hydrophone (FOPH300, Univ. Stuttgart, Germany [82]) at distance of $d \approx 1.4 \mathrm{~mm}$ above the bubble centroid. As the function of this hydrophone is based on the detection of laser light, the first strong signal is related to the light emitted from optical breakdown. After that, the two successive peaks are related to shock waves released from laser breakdown and bubble collapse, respectively. The signal is calibrated and deconvoluted by the response function of the hydrophone. 


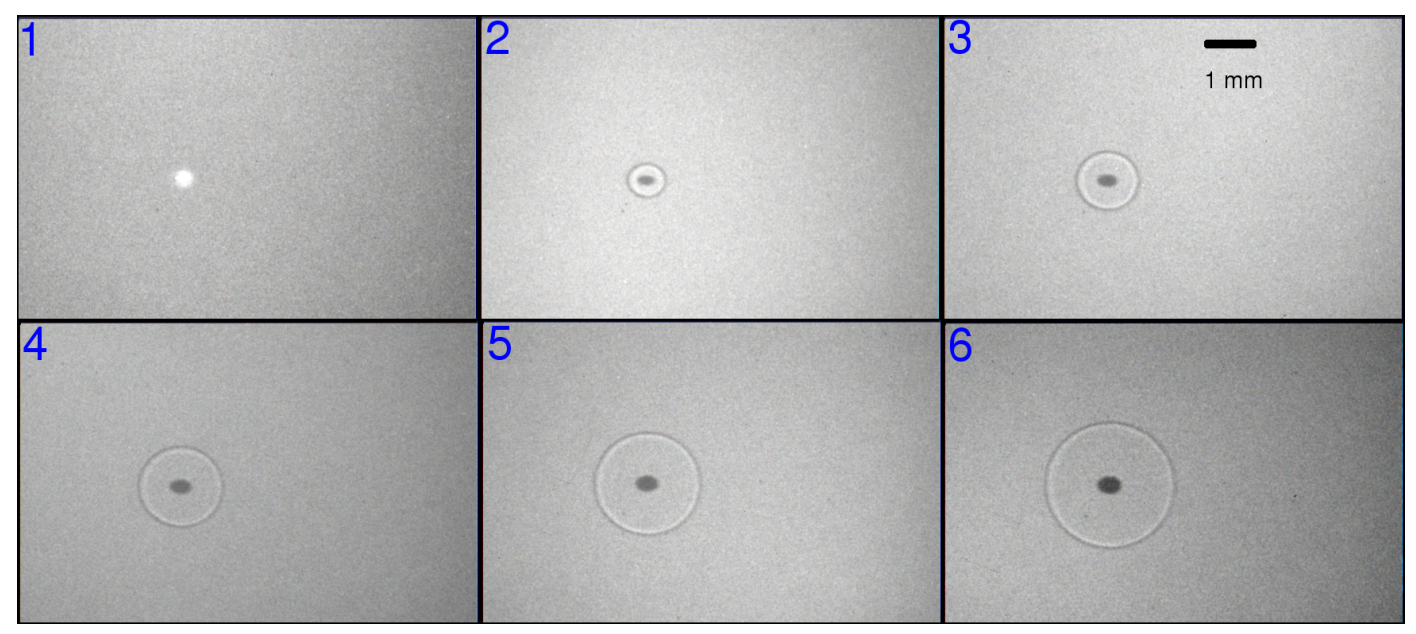

Figure 5.4: Dynamics of a cavitation bubble produced by means of optical breakdown in water at the early moments of its oscillation cycle. The first frame coincides with optical breakdown. Interframe time is $150 \mathrm{~ns}$.

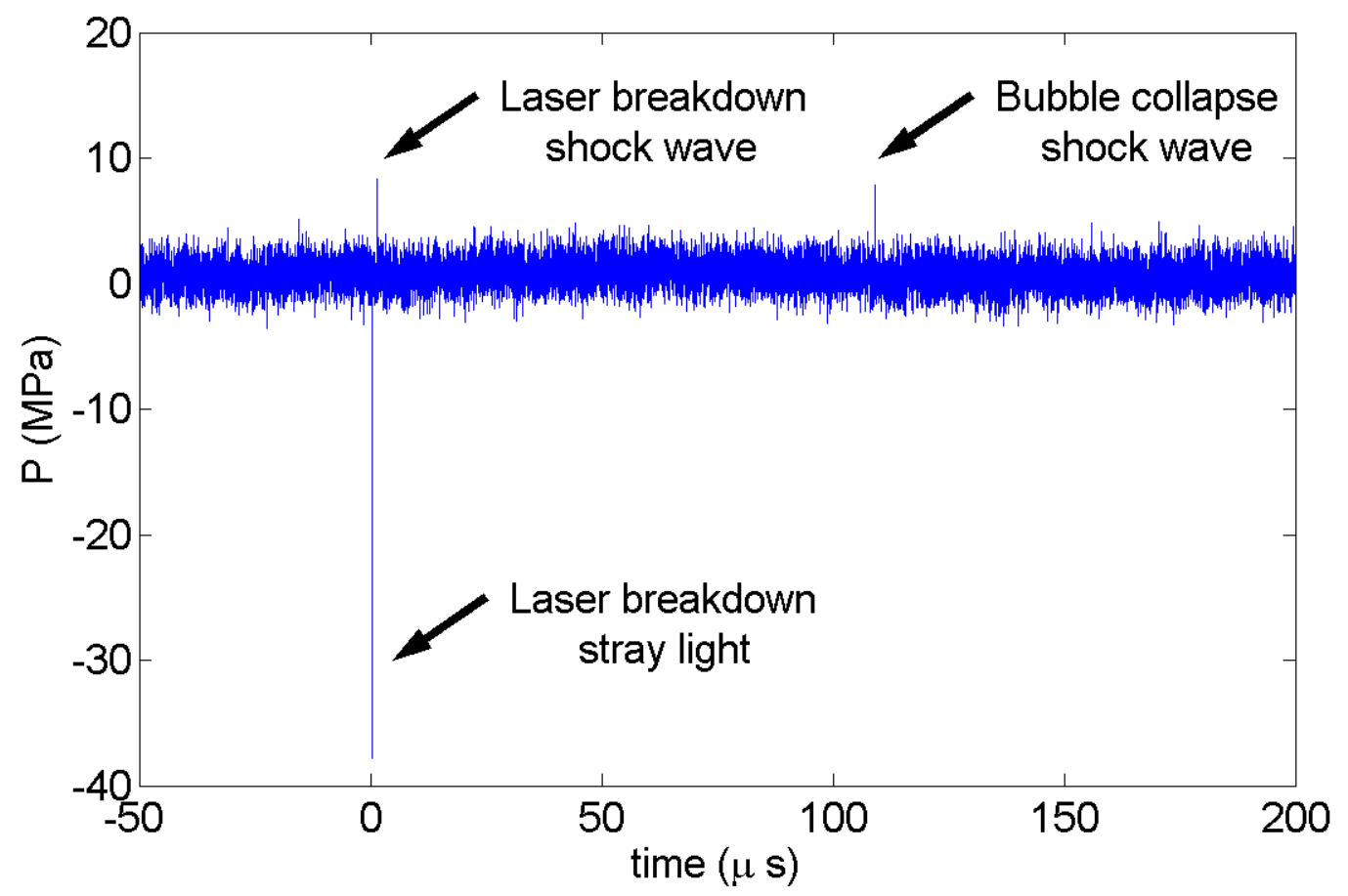

Figure 5.5: Pressure wave time history for inertial bubble dynamics measured at distance of $d \approx 1.4 \mathrm{~mm}$ above the bubble centroid. The strong negative signal is not a real pressure value. It is just due to detection of the emitted light from optical breakdown. 


\subsection{Secondary cavitation}

When a laser pulse is focused into water, because of the impurities of the medium, some microparticles absorb the laser light. Then, microbubbles will be initiated in water (weakening the tensile strength of water). They can growth in the presence of the tension tail of the shock wave profile. In Figure 5.6 the conical pattern of these so-called secondary cavitation bubbles is displayed. The laser beam enters from the right and the shock wave is propagating from bottom to the top. The laser-induced cavitation bubble is visible at the center surrounded by these secondary cavitation bubbles which can disturb the bubble dynamics. In order to reduce the population of these bubbles, some experimental measures have to be performed. In this picture the shock wave-induced jetting is also visible.

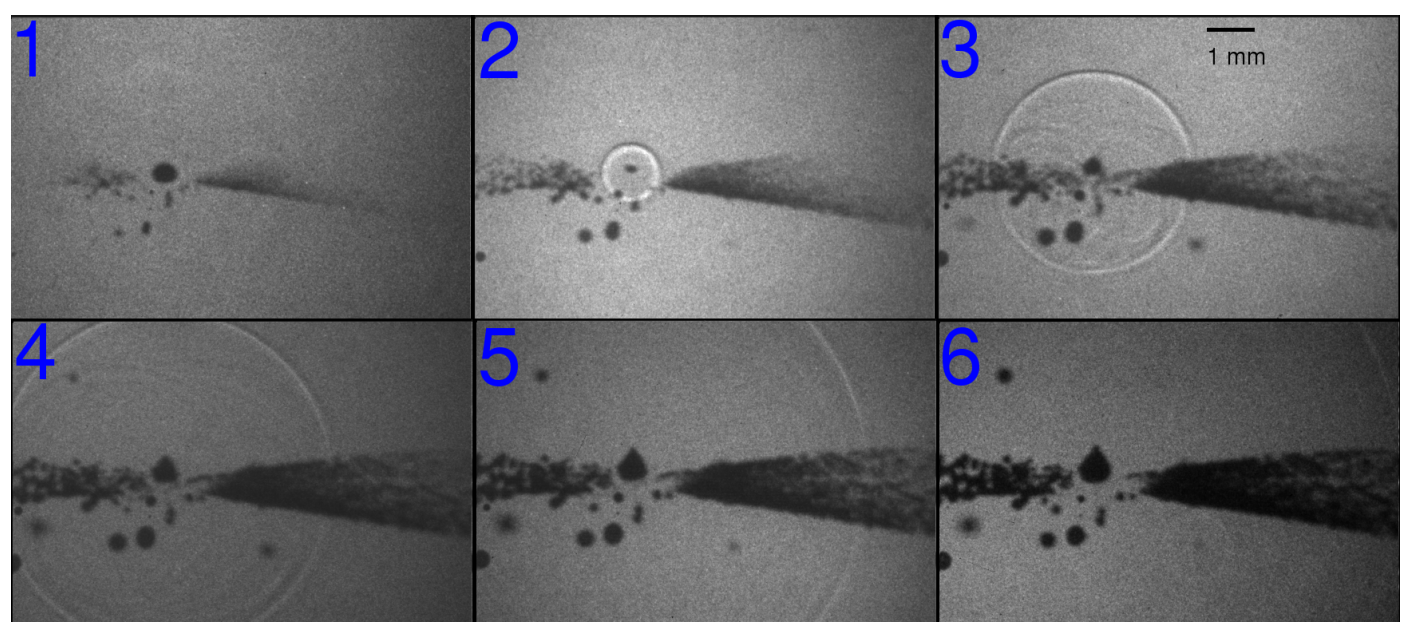

Figure 5.6: Secondary cavitation in a conical pattern. The laser beam enters from the right of each frame and the shock wave propagates from bottom to the top. 


\subsection{Pressure field measurements}

Single bubbles are initiated at the center of a water filled container, where the focus of lithotripter shock wave (LSW) is located (see Figure 4.5). After the moment of triggering of the shock wave source, it takes about $57 \mu$ s for the shock wave to reach to the center of the water tank.

The acoustic wave profile above the bubble has been measured simultaneously via a fiber optic probe hydrophone FOPH300, described in chapter 4. It records shock waves originated from optical breakdown, the lithotripter shock wave, as well as from forced bubble implosion. The hydrophone is read by a fast oscilloscope (Infinium, $1.5 \mathrm{GHz}, 8 \mathrm{GS} / \mathrm{s}$ ).

The delay generators allow wide variation of the delay time between shock wave arrival and cavitation bubble initiation. Therefore, for a certain laser energy, it is possible to adjust the times so that the lithotripter shock wave impinges on the cavity at different moments of its oscillation phase.

Shock wave intensity has been varied using 8 different intensity levels of its source. Peak pressure values for different intensity levels of the shock wave generator used in this work are illustrated in table 5.1. They are related to the measurement in the free field (in the absence of laser-induced cavitation bubbles) at distance of $d \approx 1.8 \mathrm{~mm}$ above the shock wave focus.

Table 5.1: Pressure pulse amplitudes for different intensity levels of the lithotripter shock wave source.

\begin{tabular}{cc|cc}
\hline \hline Intensity level (I) & $\mathrm{P}_{\max }(\mathrm{MPa})$ & Intensity level $(\mathrm{I})$ & $\mathrm{P}_{\max }(\mathrm{MPa})$ \\
\hline 4 & $24.4 \pm 1.1$ & 12 & $61.9 \pm 2.4$ \\
6 & $29.9 \pm 1.3$ & 14 & $79.1 \pm 2.6$ \\
8 & $37.1 \pm 1.1$ & 16 & $95.8 \pm 2.7$ \\
10 & $45.9 \pm 1.7$ & 18 & $108.1 \pm 2.5$ \\
\hline
\end{tabular}

\subsection{Timing parameters}

In the following, experimental results regarding the impingement of a LSW on laser-induced single cavitation bubbles at different moments of their oscillation phase are illustrated. Delay time between laser breakdown and moment of shock wave arrival is varied in 3 steps as $t_{i}=95,102$ and $12 \mu \mathrm{s}$. Figure 5.7 represents schematic view of the moments of LSW impact as well as forced and free bubble collapses, relative to the time of the laser breakdown, investigated in the next sections. 

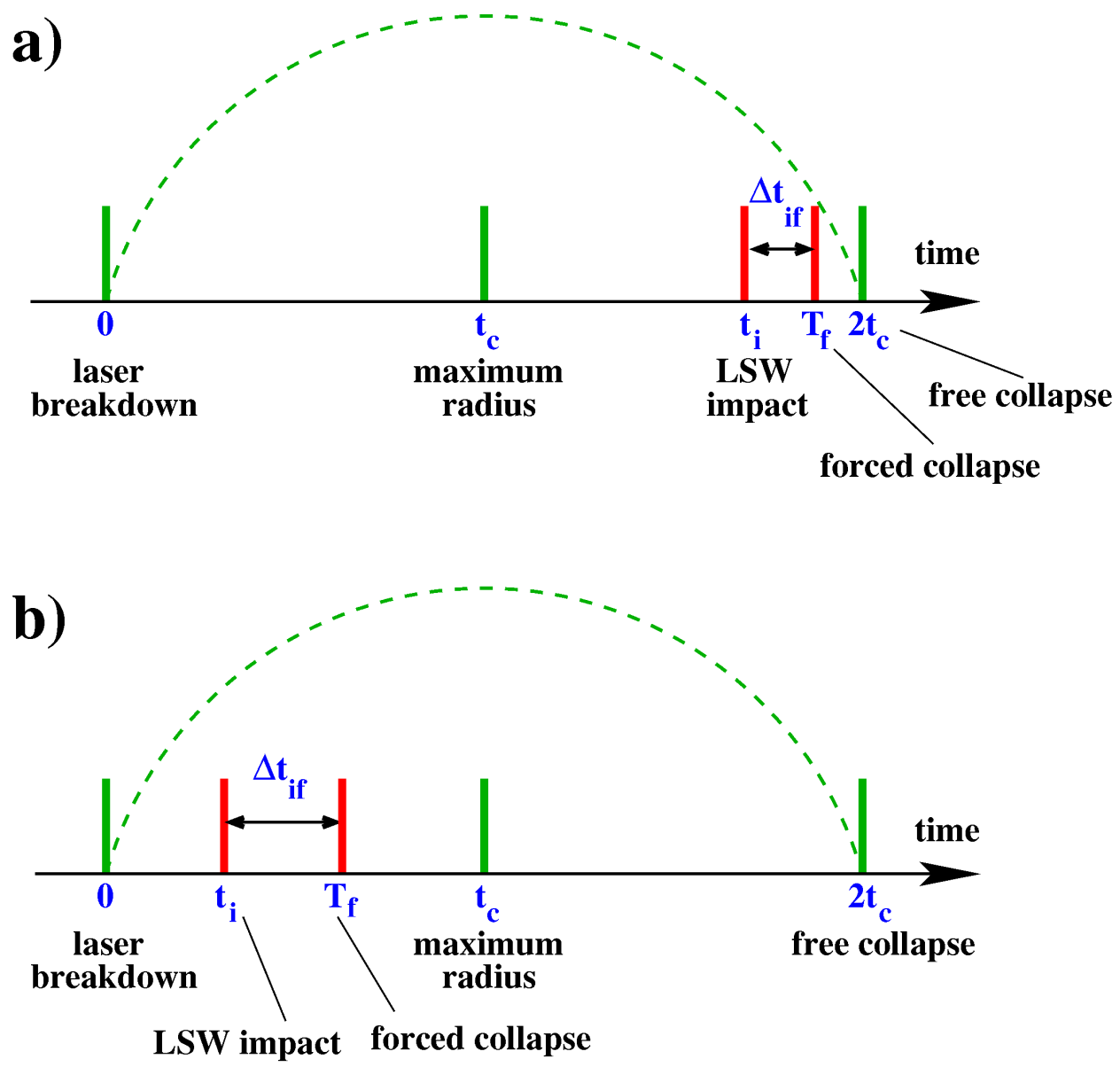

Figure 5.7: Schematic illustration of the moments of the lithotripter shock wave arrival time, forced and free bubble collapses, relative to the time of the optical breakdown (at $t=0$ ) studied in sections 5.6 and 5.7 (a) and in section 5.8 (b) (see table 5.2). The dashed graphs represent asymptotic free bubble oscillations.

Table 5.2: Definition of the parameters used for the experiments of shock wave - bubble interaction investigated in the next sections.

\begin{tabular}{c|c|c|c}
\hline \hline time $(\mathrm{t})$ & in section 5.6 & in section 5.7 & in section 5.8 \\
\hline Laser breakdown & 0 & 0 & 0 \\
LSW impact on the cavity $\left(t_{i}\right)$ & $95 \mu \mathrm{s}$ & $102 \mu \mathrm{s}$ & $12 \mu \mathrm{s}$ \\
Free bubble oscillation period $\left(2 t_{c}\right)$ & $104.3 \mu \mathrm{s}$ & $104.7 \mu \mathrm{s}$ & $101.9 \mu \mathrm{s}$ \\
Forced bubble oscillation period $\left(T_{f}\right)$ & $t_{i}+\Delta t_{i f}$ & $t_{i}+\Delta t_{i f}$ & $t_{i}+\Delta t_{i f}$ \\
Normalized LSW arrival time $\left(\alpha=t_{i} / 2 t_{c}\right)$ & 0.91 & 0.97 & 0.12 \\
\hline
\end{tabular}

The value of the parameter $\Delta t_{i f}$ is a function of the shock wave energy as well as the time of LSW impingement on the bubble and will be discussed later. 


\subsection{Experimental results: Part I}

The oscillation period of the free inertial cavitation bubbles studied in this step is $2 t_{c}=104.3 \pm 3.2 \mu \mathrm{s}$ (from 40 measurements). The behavior of lasergenerated single cavitation bubble under the influence of a lithotripter shock wave (LSW) is shown in Figure 5.8. This image is a shadowgraph picture of single bubbles with exposure time of $20 \mathrm{~ns}$ and interframe time of $0.6 \mu \mathrm{s}$. The shock wave propagates from bottom to top for each image sequence and the laser beam is coming from the right. The LSW impinges on the cavitation bubble on the first frame, which corresponds to the moment at $t_{i}=95 \mu$ s after the optical breakdown (see Figure 5.7). The normalized time of shock wave impact is $\alpha=t_{i} / 2 t_{c}=0.91$, which shows that the initial bubble it at its collapse phase. The delay time between the shock wave source trigger and the moment of observation is $\Delta t \approx 56.6 \mu \mathrm{s}$, which is the time that the LSW needs to reach to the focus position. This delay time is kept constant also when using different intensity levels of the shock wave generator.

The shock wave intensity is the same for each image sequence (row in Figure 5.8) and increases from top to the bottom (from $a$ to $h$ ). The shock amplitude is varied in 8 steps. The shock wave peak pressure measured at a distance of $d \approx 1.8 \mathrm{~mm}$ above the focus location in the free field (no bubble) ranges from $P_{\max }=24.4 \pm 1.1 \mathrm{MPa}\left(a\right.$ in Figure 5.8) to $P_{\max }=108.1 \pm 2.5(h$ in Figure 5.8). At the moment of the shock wave impact, the vertical bubble diameter is $D_{v 0}=0.80 \pm 0.02 \mathrm{~mm}$, while the bubble horizontal diameter is $D_{h 0}=0.76 \pm 0.02 \mathrm{~mm}$.

Each image sequence ( $a$ to $h$ in Figure 5.8) consists of two subsequences of 6 pictures with delay time of $\Delta t=0.6 \mu \mathrm{s}$. Therefore, for every image series, two bubbles are generated. Regarding a specific shock wave energy (row in Figure 5.8), the corresponding image sequence is selected out of several records in order to minimize the difference between the forced bubble collapse times for the two subsequences. It is worth noting that secondary cavitation due to the tension part of the pressure profile, disturbs the laser-generated bubble dynamics in many cases, although deionized, filtered and partially degassed water has been used. As depicted in Figure 5.8, after being exposed to the shock, the bubble loses its spherical symmetry. It collapses to a disk shape perpendicular to the direction of the propagation of the lithotripter shock wave. A high speed liquid jet penetrates into the bubble's inner part and develops in the direction of the shock wave passage. By increasing the shock wave energy (from top to the bottom in Figure 5.8), the forced collapse occurs earlier relative to the moment of the shock impingement (first frame).

Enhancement of the secondary shock wave emitted from the bubble collapse is discussed in 5.6.1. Reduction of the forced bubble collapse time compared to free inertial oscillation, is considered in 5.6.2. LSW pulse amplitude for different shock wave energies in the presence of single cavitation bubbles is characterized in 5.6.3. Liquid jet velocities as well as the migration of the bubble centroid in the direction of the LSW passage are described in 5.6.4. 


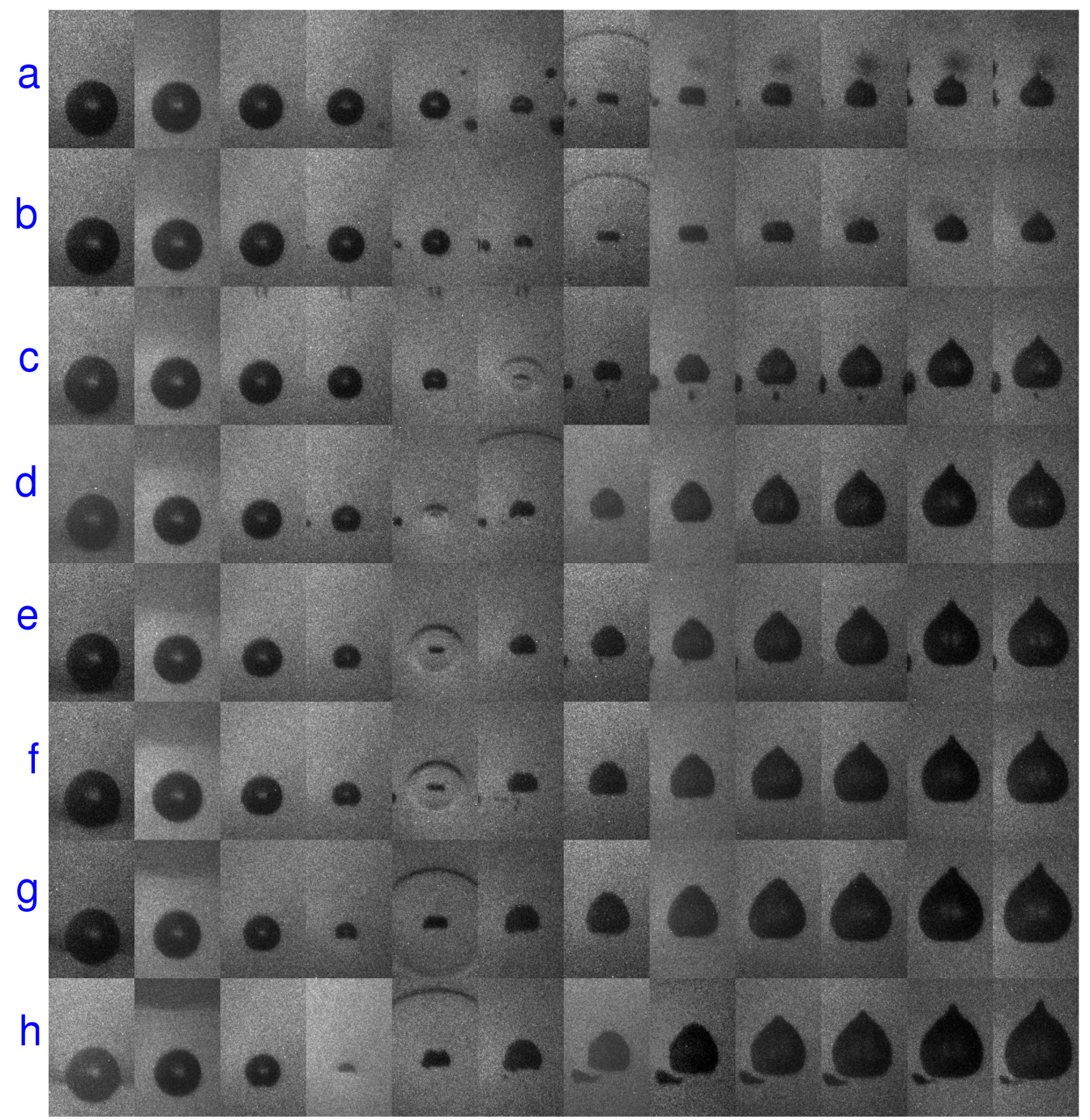

Figure 5.8: High-speed image sequences of interaction between lithotripter shock wave and laser-induced single cavitation bubbles. Normalized time of shock wave impingement is $\alpha=0.91$. Interframe time is $0.6 \mu$ s and exposure time is $20 \mathrm{~ns}$. Frame width and height are $1.2 \mathrm{~mm}$ and $1.9 \mathrm{~mm}$, respectively. The shock wave propagates from bottom to the top of each frame and impinges on the cavity on the first frame in each row. The shock strength is increasing from $a$ to $h$ and is kept constant for every image sequence (row). The peak pressures of the shock wave measured at the distance of $d \approx 1.8 \mathrm{~mm}$ above the shock focus in the free field (no bubble) are $P_{\max }=24.4(a), 29.9(b), 37.1(c), 45.9(d), 61.9(e), 79.1(f), 95.8(g)$, 108.1(h) MPa. Initial bubble diameter in vertical direction is $D_{v 0}=0.80 \pm 0.02 \mathrm{~mm}$ and its initial horizontal diameter is $D_{h 0}=0.76 \pm 0.02 \mathrm{~mm}$. The laser beam enters from the right. 
In Figure 5.9, the lithotripter shock wave (LSW) and forced collapse shock are shown for different LSW intensities. The shock wave source voltages are $V_{b}=2.53 \mathrm{kV}(I=4), V_{b}=3.56 \mathrm{kV}(I=10), V_{b}=4.63 \mathrm{kV}(I=16)$. The measurement is performed at a distance of $d \approx 1.8 \mathrm{~mm}$ above the shock focus position. Signals are calibrated and deconvoluted by the response function of fiber optic probe hydrophone discussed in chapter 4. By increasing the LSW intensity, the cavity implosion occurs earlier with a stronger amplitude.
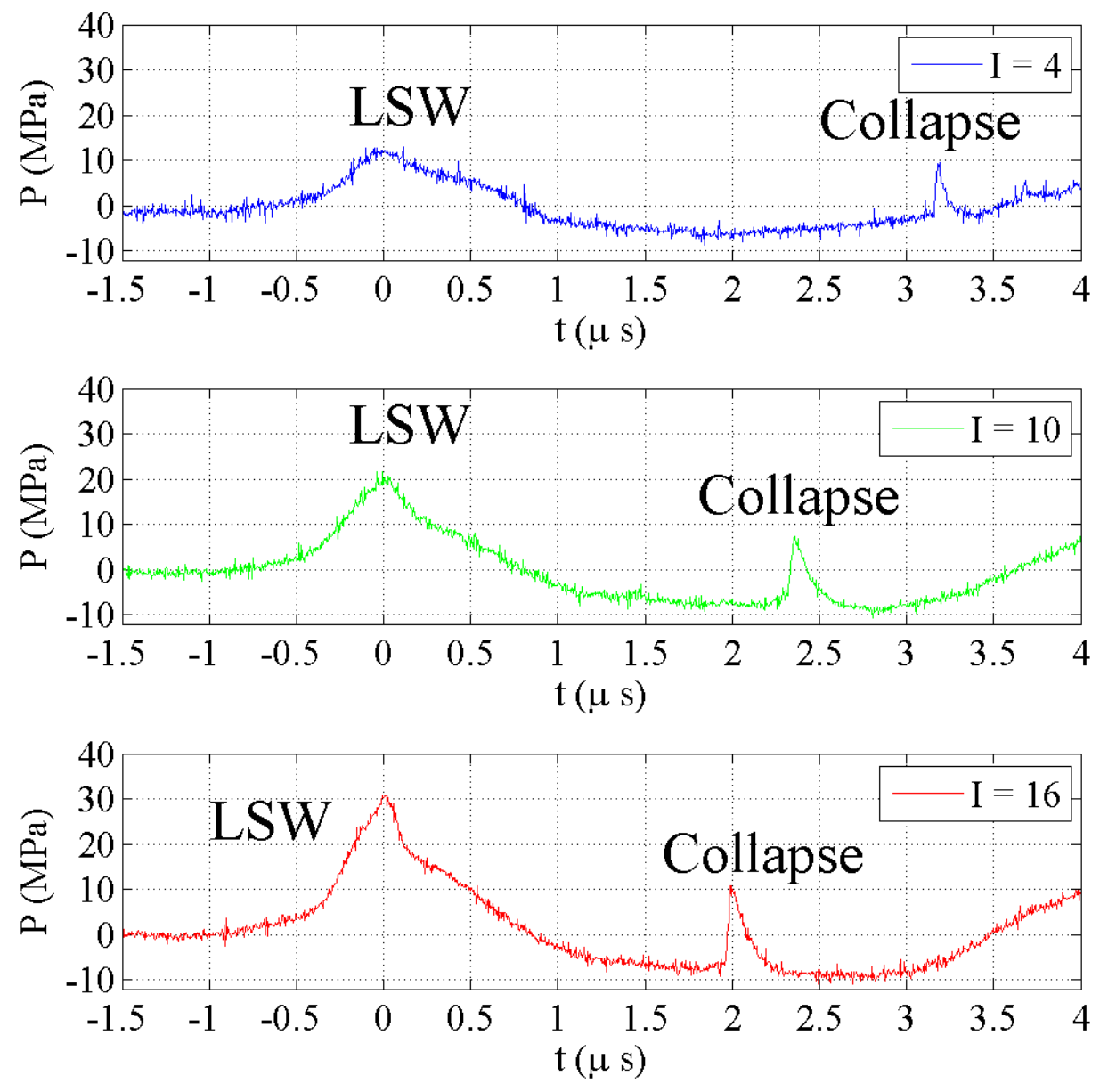

Figure 5.9: Typical LSW and collapse shock wave profile for three intensity levels of the shock wave generator. The moment of $t=0$ coincides with the peak pressure wave related to LSW. The second maximum represents the forced bubble collapse.

It is worth mentioning that the solid lines plotted in the next graphs represent polynomial fits to the experimental data to guide the eye. 


\subsubsection{Pressure enhancement}

The shock wave amplitude from inertial single cavity collapse according to 40 measurements is $P_{c}^{\prime}=7.3 \pm 1.1 \mathrm{MPa}$, with full width at half maximum (FWHM) based on 15 measurements being $\tau_{c}=44.0 \pm 5.1 \mathrm{~ns}$.

Parameter $R$ is defined as the ratio between peak pressure wave emitted from the forced bubble collapse $P_{c}$ and that of inertial cavity implosion $P_{c}^{\prime}$. This variable indicates amplification strength of the forced collapse shock wave due to application of the lithotripter shock wave. The pressure enhancement for different LSW energies is displayed in Figure 5.10. Errors of the acoustic field measurements are derived from the bias noise level of the hydrophone signal, which is the most prominent source of uncertainty. As illustrated in Figure 5.10, the collapse shock amplitude $P_{c}$ becomes stronger by increasing the LSW intensity (increasing of the shock wave generator maximum voltage $\left.V_{b}\right)$. The pressure amplification factor $\left(R=P_{c} / P_{c}^{\prime}\right)$ is between $R \approx 1.6$ for $V_{b}=2.53 \mathrm{kV}$ and $R \approx 2.7$ for $V_{b}=4.99 \mathrm{kV}$. As it can be seen, increasing the shock wave generator peak voltage by a factor of about 2 , leads to a rise in the enhancement factor $R$ by $\approx 1.7$ times.

The cavitation bubble collapse occurs in the tension phase of the LSW profile (see Figure 5.9). Even though the positive part of the shock wave profile urges the cavity to collapse, the succeeding negative phase tends to retard the bubble implosion. For the cases that the collapse happens earlier (using higher LSW amplitudes), the cavity has a lower chance to be influenced by the tension part of the LSW profile. Therefore, the retardation has a minor effect and the forced collapse is more violent with stronger resultant collapse shock wave pressure.

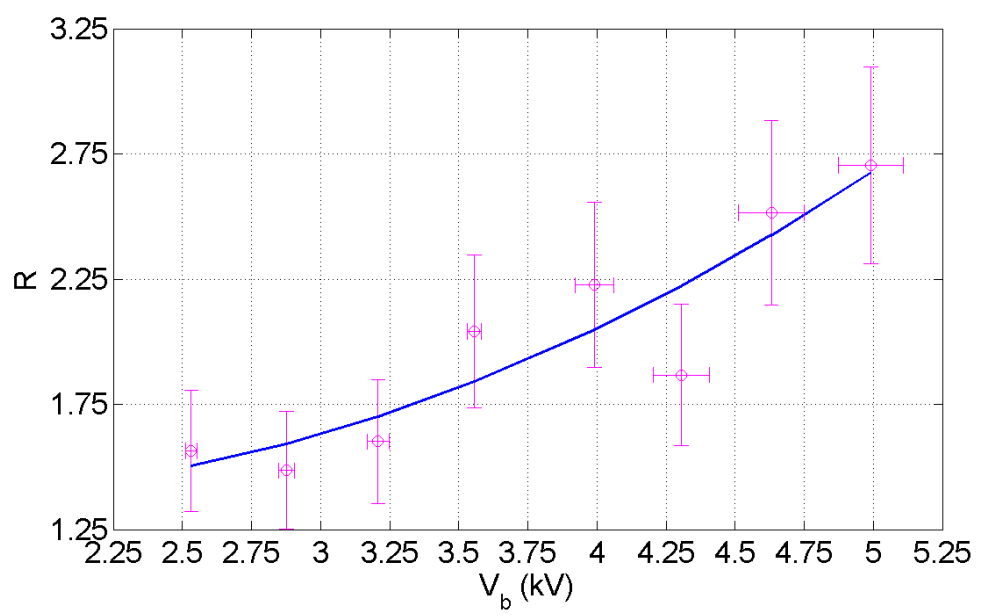

Figure 5.10: Amplification of the shock wave emitted from the forced bubble collapse due to the application of the lithotripter shock waves $\left(R=P_{c} / P_{c}^{\prime}\right)$. Error bars are related to the standard deviation of the experimental data. The solid line illustrates a quadratic fit to the measurement results. 


\subsubsection{Reduction of collapse time}

In general, a significant reduction of bubble collapse time can be observed, if the shock wave energy is strong enough to result in a forced cavity implosion. It is due to the energy which is transferred from LSW to the bubble. The time interval between lithotripter shock wave arrival and forced bubble collapse $\Delta t_{i f}$, is plotted in Figure 5.11 for different peak voltages of the shock wave source $V_{b}$. As illustrated in this picture, the parameter $\Delta t_{i f}$ is reduced more by increasing the external shock wave intensity.

Increasing of the peak voltage from $V_{b} \approx 2.5 \mathrm{kV}$ to $V_{b} \approx 5.0 \mathrm{kV}$, the time interval $\Delta t_{i f}$ is reduced by an amount of about $1.3 \mu \mathrm{s}$ (from $3.2 \mu \mathrm{s}$ to about $1.9 \mu \mathrm{s})$. Reduction of the parameter $\Delta t_{i f}$ takes place faster for shock wave generator peak voltages in the range less than about $4.0 \mathrm{kV}$, while there is a smoother behavior for the maximum voltages higher than that value.

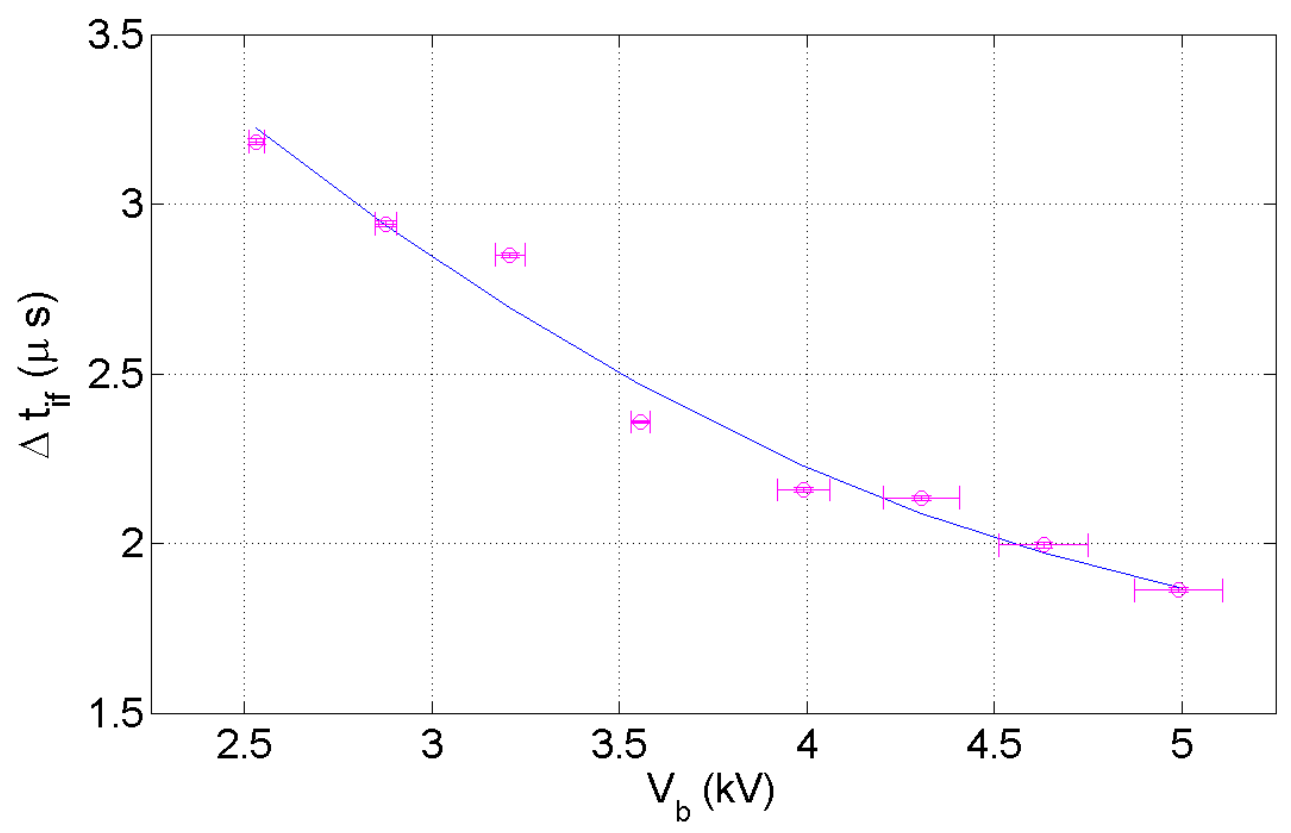

Figure 5.11: Reduction of bubble implosion time due to interaction between LSW and laser-generated single bubbles. Parameter $\Delta t_{i f}$ is the time interval between lithotripter shock wave (LSW) impingement on the bubble and the forced bubble collapse. Error bars correspond to standard deviation of the experimental data. The solid line is obtained by a quadratic fit to the experimental results. 


\subsubsection{Lithotripter shock wave (LSW) pulse amplitude}

Pulse amplitude of LSW measured above the laser-induced cavitation bubbles is derived by smoothing of the shock wave profile with a span of 3 data points. Then, the maximum value of the smoothed signal has been taken into account.

Figure 5.12 depicts the LSW pulse amplitude in presence of cavitation bubbles for different shock energies. The measurement is performed at $d \approx 1.8 \mathrm{~mm}$ above the shock wave focus location. In this Figure, it is visible that there is a nearly linear relation between the shock wave generator peak voltage $V_{b}$ and the LSW pulse amplitude. The equation of the fitted line to the measured values is: $P_{L S W}(\mathrm{MPa})=(8.74 \pm 0.22) V_{b}(\mathrm{kV})-8.97 \pm 0.84$. The pulse amplitude above the bubble increases from $P_{L S W} \approx 13.1 \mathrm{MPa}$ to $P_{L S W} \approx 34.6 \mathrm{MPa}$ for shock wave generator peak voltages ranging from $V_{b} \approx 2.5 \mathrm{kV}$ to $V_{b} \approx 5.0 \mathrm{kV}$.

Due to the presence of the single cavitation bubbles, the lithotripter shock wave pulse amplitude is weaker than that in the free field. The amount of reduction in LSW peak pressure is derived by introducing weakness factor as $\left(R_{L S W}=1-\frac{P_{L S W}}{P_{L S W}^{\prime}}\right)$. Here $P_{L S W}$ is the LSW maximum pressure in presence of the bubble and $P_{L S W}^{\prime}$ being the shock wave pulse amplitude in the free field (without the laser-generated single cavitation bubbles).

Damping of the LSW pulse amplitude due to the existence of single cavitation bubbles in the pressure wave field, is illustrated in Figure 5.13. The amplitude of the shock wave is reduced more by using higher intensity levels of the shock wave source. The amount of reduction in LSW peak pressure ranges from $R_{L S W}=46.28 \%$ to $R_{L S W}=68.02 \%$ for $V_{b} \approx 2.5 \mathrm{kV}$ to $V_{b} \approx 5.0 \mathrm{kV}$.

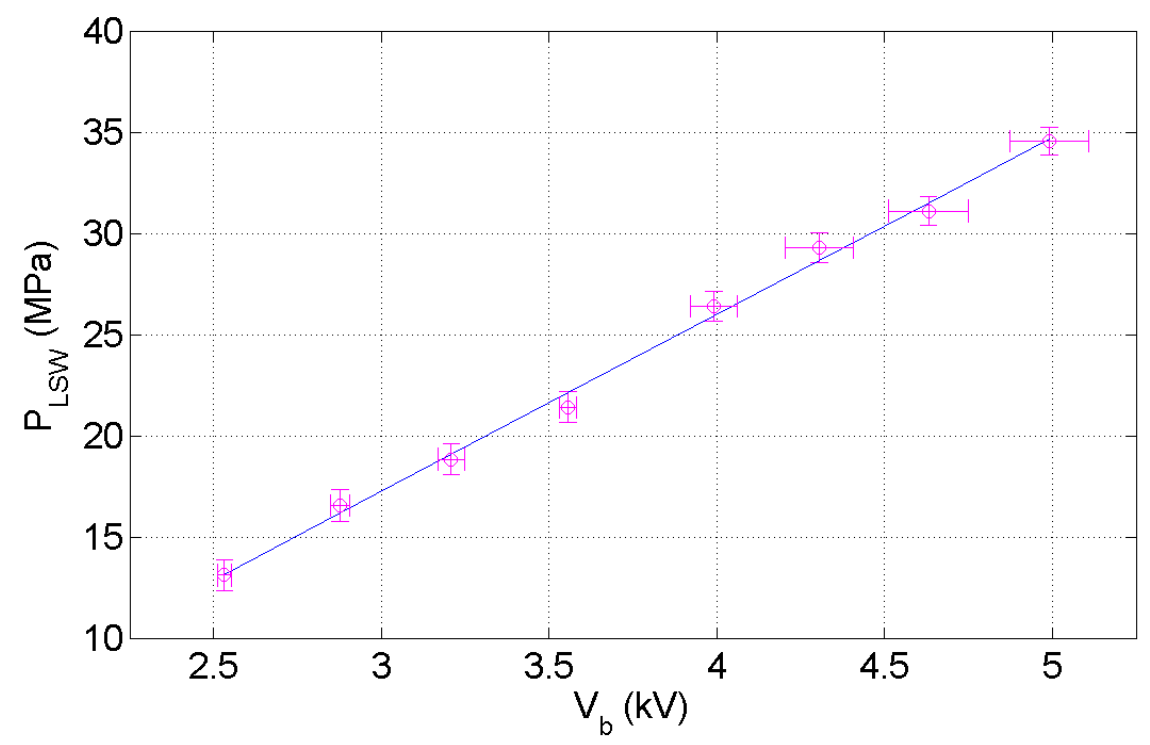

Figure 5.12: Lithotripter shock wave (LSW) peak pressure in presence of the laser-generated single bubbles. The solid line displays a linear fit to the experimental data. 


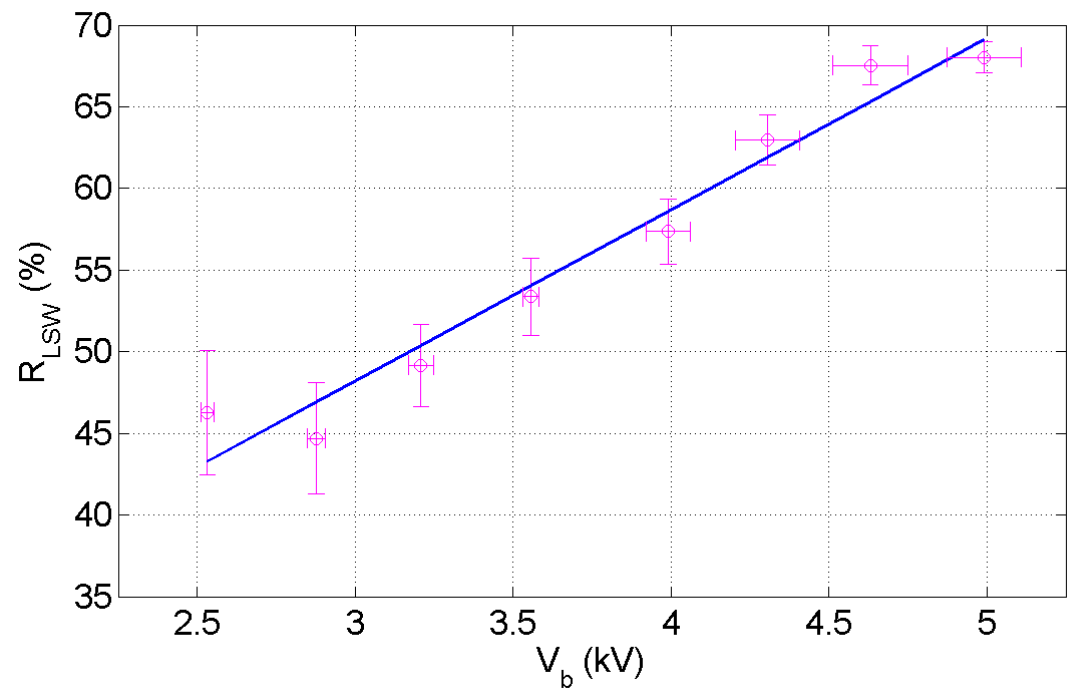

Figure 5.13: Reduction of LSW peak amplitude due to the presence of the laser-generated single cavitation bubbles. The solid line represents a linear fit to the experimental data $\left(R_{L S W}=(10.49 \pm\right.$ $\left.0.88) V_{b}+16.70 \pm 3.39\right)$. Error bars correspond to the standard deviation of the measurements results.

Figure 5.14 illustrates the effect of the presence of a bubble on the LSW time history. In this picture, for a specific intensity level of the shock wave generator $(I=18)$, shock wave signals with and without existence of a single cavitation bubble are plotted. Full width at half maximum (FWHM) of the LSW profile under the effect of the bubble, is about 4 times that of the free signal.

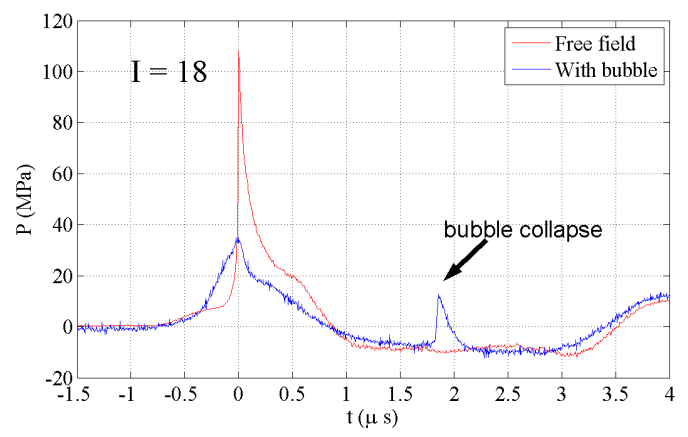

Figure 5.14: Comparison of the lithotripter shock wave (LSW) profile in presence and absence of a laser-generated single bubble. The signal becomes broadened in response to the existence of single bubbles, because of both diffraction and absorption effects. 


\subsubsection{Bubble dynamics under the influence of the applied lithotripter shock wave (LSW)}

Asymmetric deformation of the single cavitation bubbles due to the LSW impingement can be evaluated by the amount of deviation of bubble diameters in vertical and horizontal directions $\left(D_{v}\right.$ and $\left.D_{h}\right)$ from each other. It is worth mentioning that the vertical direction is the same as the direction of the shock wave passage. Figure 5.15 represents the time rate of variation of parameters $D_{v}$ and $D_{h}$ relative to their initial values $D_{v 0}$ and $D_{h 0}$. Initial values are related to the moment of LSW impact. This time coincides with the first column in Figure 5.8 and $t=0$ in Figure 5.15. Experimental results depicted in Figure 5.15 are related to setting of two values for peak voltage of the shock wave source as $V_{b} \approx 2.5 \mathrm{kV}$ and $V_{b} \approx 5.0 \mathrm{kV}$. In this Figure, the effect of variation of the shock wave energy on the dynamics of the bubble in vertical and horizontal directions is observed.

The time interval between the moment of cavity collapse and $3 \mu$ s later is considered for temporal variation of bubble diameters. For $V_{b} \approx 2.5 \mathrm{kV}$ (intensity level $I=4$ ), dimensionless vertical bubble diameter $R_{1}=D_{v} / D_{v 0}$ varies by a time rate of $\approx 0.15 / \mu \mathrm{s}$. But for dimensionless bubble diameter in horizontal direction $R_{2}=D_{h} / D_{h 0}$ this rate is about $0.08 / \mu \mathrm{s}$. For the case of using the intensity level $I=18\left(V_{b} \approx 5.0 \mathrm{kV}\right)$, parameter $R_{1}$ rises faster $(\approx 0.30 / \mu \mathrm{s})$ and for $R_{2}$ the rate of its time variation is $\approx 0.19 / \mu \mathrm{s}$.

The rate of time variation for both vertical and horizontal bubble diameters is increasing with shock wave energy. It can be seen that for both shock intensities, the temporal variation of $D_{v}$ is stronger than that of $D_{h}$. This fact indicates that the bubble collapses and rebounds non-spherically and its elongation in the vertical direction is more than its horizontal one. Therefore, the bubble collapse in the direction of the LSW propagation is more violent with induction of a liquid jet toward the bubble interior. Finally, the liquid jet develops in the direction of shock wave passage.

The accuracy of the given values is limited by temporal and spatial resolution of high-speed images. The time interval between two data points is $0.6 \mu \mathrm{s}$. 

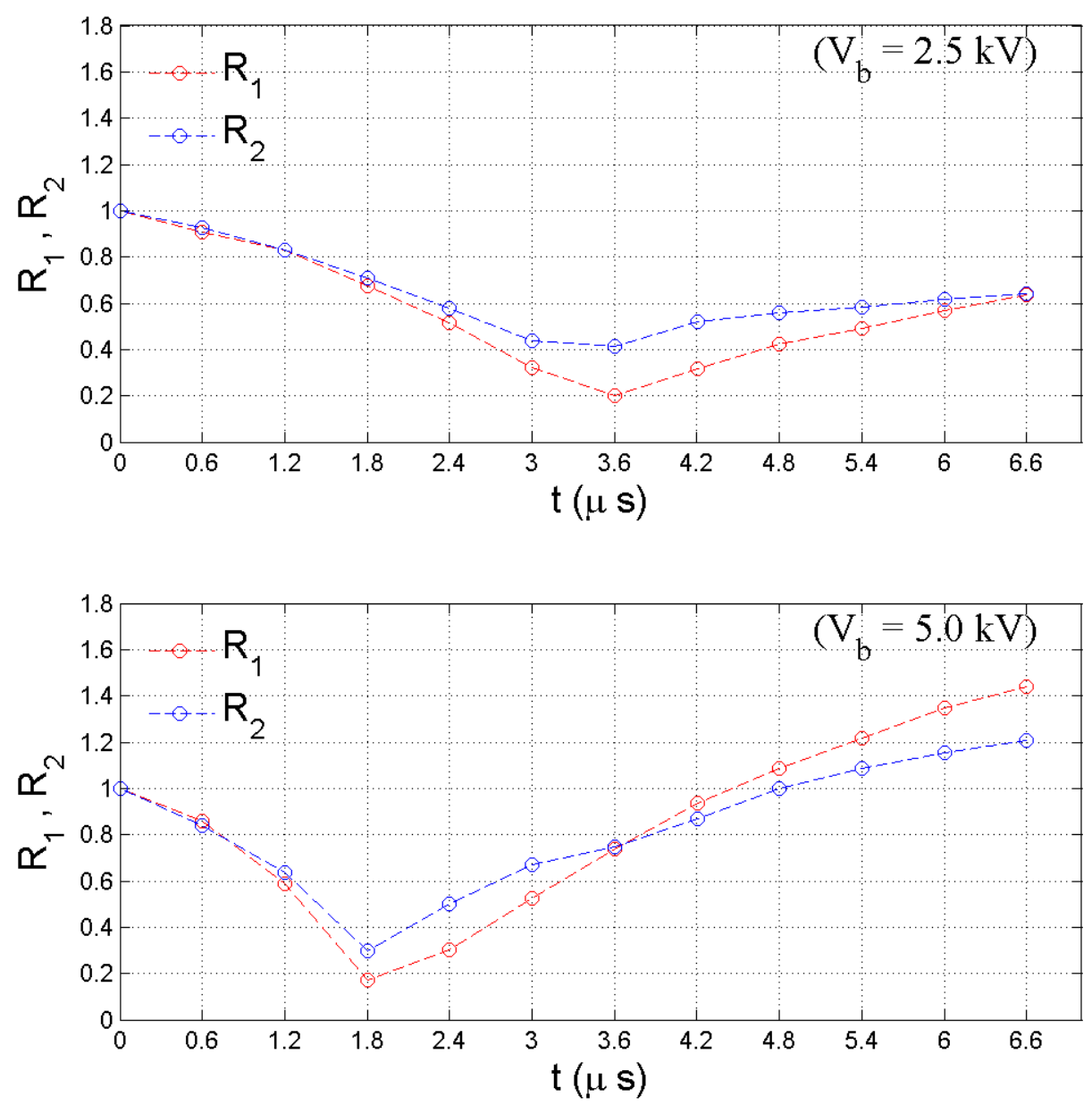

Figure 5.15: Vertical and horizontal diameter of laser-generated single cavitation bubbles relative to their initial values, $R_{1}=D_{v} / D_{v 0}$ and $R_{2}=D_{h} / D_{h 0}$. The lithotripter shock wave (LSW) impinges on single cavities at the moment of $t=0$. The results are related to two intensity levels of the shock wave source $(I=4$ and $I=18)$. Parameter $V_{b}$ is the peak voltage of the shock wave generator. For a specific amount of shock wave energy, the temporal variation of bubble vertical diameter is larger than that of horizontal orientation after the moment of minimum sizes. By increasing the shock wave energy, the bubble dynamics becomes more violent. 
The distances of lower and upper bubble walls from the top of each frame in Figure 5.8 at different moments of bubble dynamics are plotted in Figure 5.16. This Figure illustrates graphs related to applying $V_{b} \approx 2.9 \mathrm{kV}$ and $V_{b} \approx 4.6 \mathrm{kV}$. In this step, the liquid jet velocities from analyzing of the image sequences in Figure 5.8 are derived. For estimation of the jet velocity, two procedures have been performed. First, by recording the difference in the spatial position of the lower bubble wall before cavity collapse and upper bubble wall after that [73]. This difference is divided by the time interval between two adjacent frames in Figure 5.8 which is $\Delta t=0.6 \mu \mathrm{s}$. In this case, the maximum jet velocity is calculated.

Using this method, maximum liquid jet velocities for $V_{b} \approx 2.9 \mathrm{kV}$ and $V_{b} \approx$ $4.6 \mathrm{kV}$ are $V_{j} \approx 380 \pm 70 \mathrm{~m} / \mathrm{s}$ and $V_{j} \approx 530 \pm 70 \mathrm{~m} / \mathrm{s}$, respectively. The errors are basically due to the uncertainties in measurements of the bubble wall positions. They arise because of the drawbacks in focusing by microscope and camera system, limited resolution of the Imacon camera $(576 \times 384$ pixels $)$ and illumination systems. Temporal resolution of the experimental data is limited to $0.6 \mu \mathrm{s}$. It can be seen that increasing the shock wave source voltage from $V_{b} \approx 2.9 \mathrm{kV}$ to $V_{b} \approx 4.6 \mathrm{kV}$ enhances the maximum liquid jet velocity by a factor of about 1.4. The second method is described on the next page.
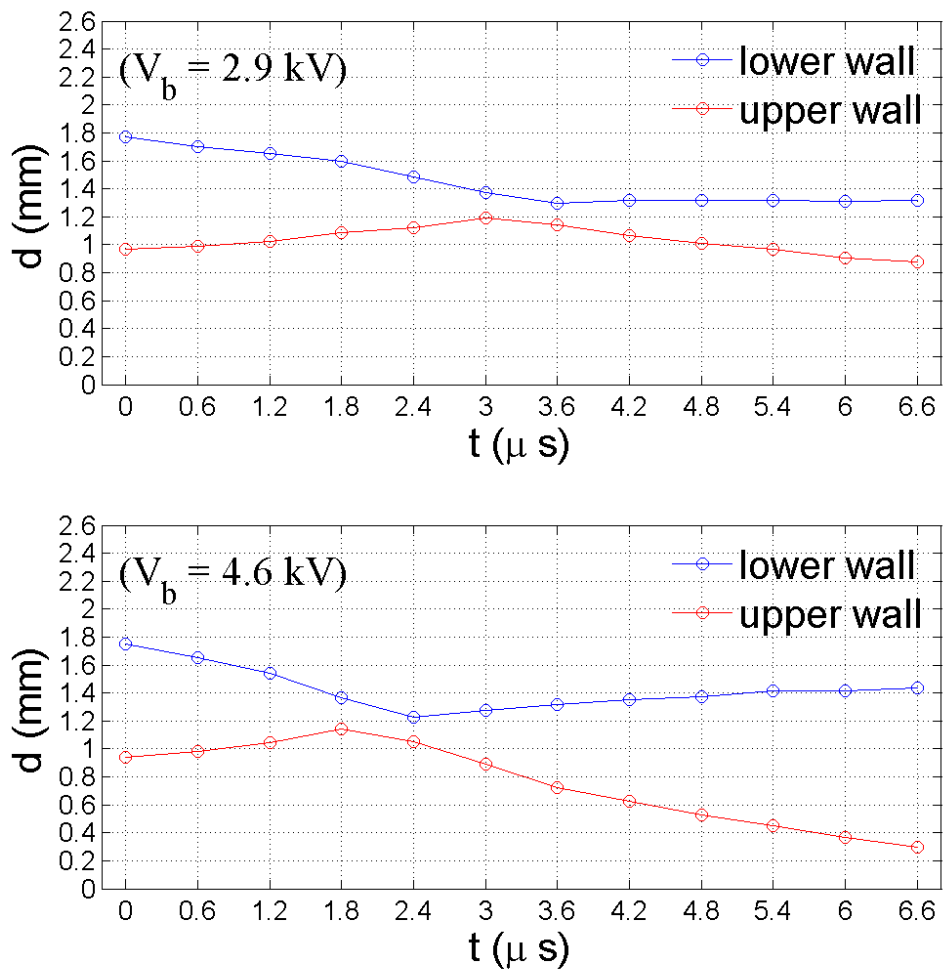

Figure 5.16: Temporal variation of distance $(d)$ of the lower and upper bubble walls from the top of each frame in Figure 5.8. 
The spatial position of the bubble tip $Y_{t}$ after the moment of collapse, relative to its location at this time $\left(Y_{t}(0)=0\right)$, is illustrated in Figure 5.17. These typical experimental results are related to application of two different shock wave energies $\left(V_{b}=2.9 \mathrm{kV}\right.$ and $\left.V_{b}=4.6 \mathrm{kV}\right)$.

As the second method, the bubble tip velocities are measured by the difference in the position of the cavity tip at the moment of implosion and $3 \mu \mathrm{s}$ later. In this case, the bubble tip velocities are $V_{t} \approx 90 \mathrm{~m} / \mathrm{s}$ and $V_{t} \approx 200 \mathrm{~m} / \mathrm{s}$ for $V_{b} \approx 2.9 \mathrm{kV}$ and $V_{b} \approx 4.6 \mathrm{kV}$, respectively. These values are average velocities over $\Delta t=3 \mu \mathrm{s}$, which are far below the maximum liquid jet velocities derived by the first method $\left(V_{j} \approx 380\right.$ and $\approx 530 \mathrm{~m} / \mathrm{s}$ ). Damping of the jet is probably due to the viscosity of the liquid [73]. As shown in Figure 5.17, the bubble tip travels in the direction of shock wave propagation with a higher velocity by rising the shock wave energy. Deviation of the two graphs is increasing with time.

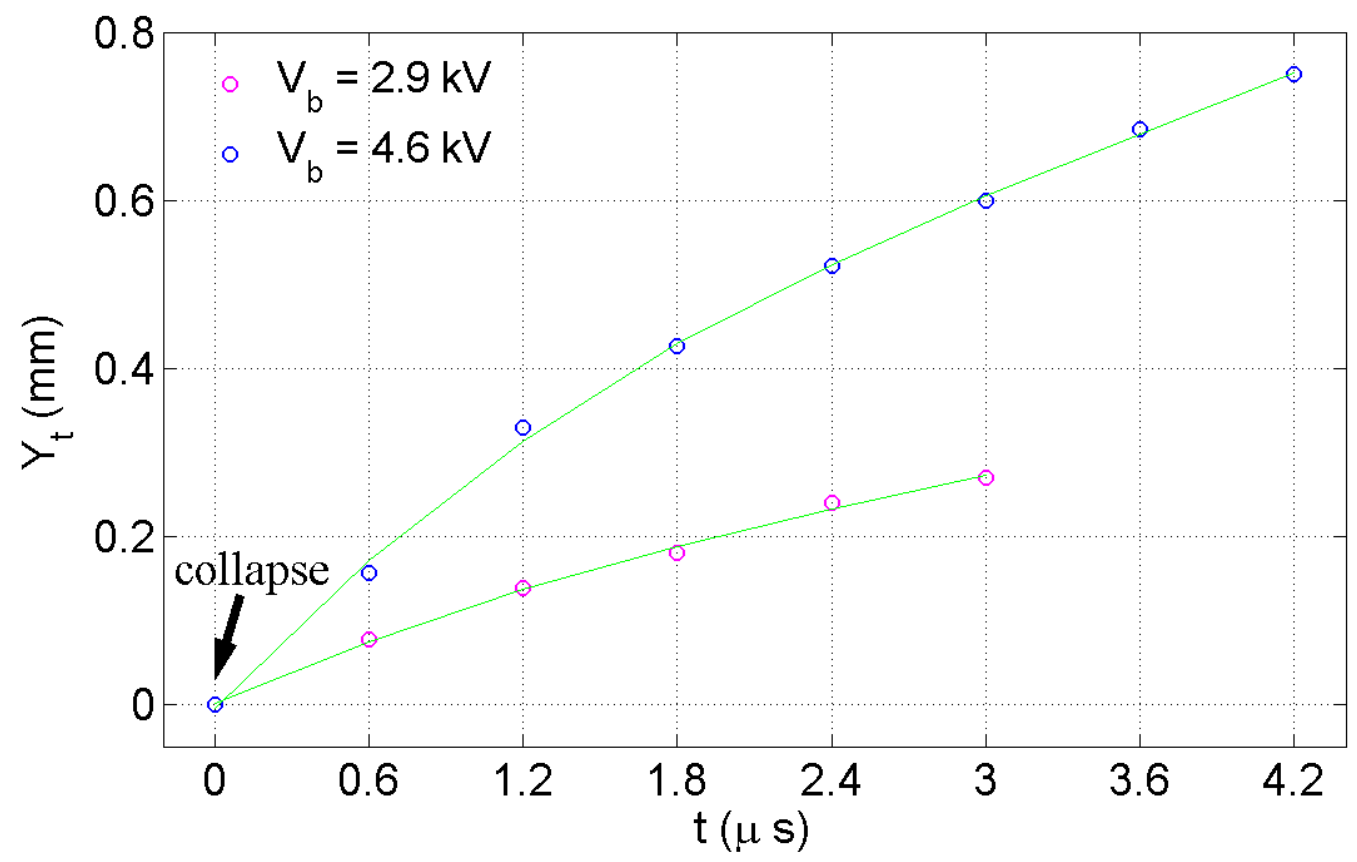

Figure 5.17: Relative position of the bubble tip. Moment of zero coincides with cavity collapse. The zero in $Y_{t}$ indicates the position of the bubble tip at this time. The solid lines represent cubic fits to the experimental data. 
When an external shock wave impinges on a cavitation bubble, work is done by the shock. Therefore, there is a momentum transfer from the shock wave to the bubble which leads to migration of the bubble centroid $Y_{C}$ in the direction of the shock wave propagation. This orientation is from bottom to top of each frame in Figure 5.8.

The relative bubble centroid migration is shown in Figure 5.18 for application of 6 different LSW amplitudes. The applied shock wave source maximum voltages range from $2.9 \mathrm{kV}$ to $5.0 \mathrm{kV}$. The initial time coincides with the first frame in Figure 5.8, which is the moment of the shock wave impingement. $Y_{C}=0$ relates to location of the bubble centroid in vertical direction at the moment of $t=0$.

As it can be seen in this picture, for the first $0.6 \mu$ s after the shock wave impact, there is almost no significant movement of the bubble centroid for all shock wave generator voltages. The slope of $Y_{C}$ is increasing with shock wave generator peak voltage $V_{b}$. At the time $t=6.6 \mu$ s after the LSW impact, the bubble can be moved to up to about $0.5 \mathrm{~mm}$.

The bubble centroid velocity $V_{C}$ is derived by averaging over $\Delta t=3.0 \mu \mathrm{s}$ and compiled in table 5.3.

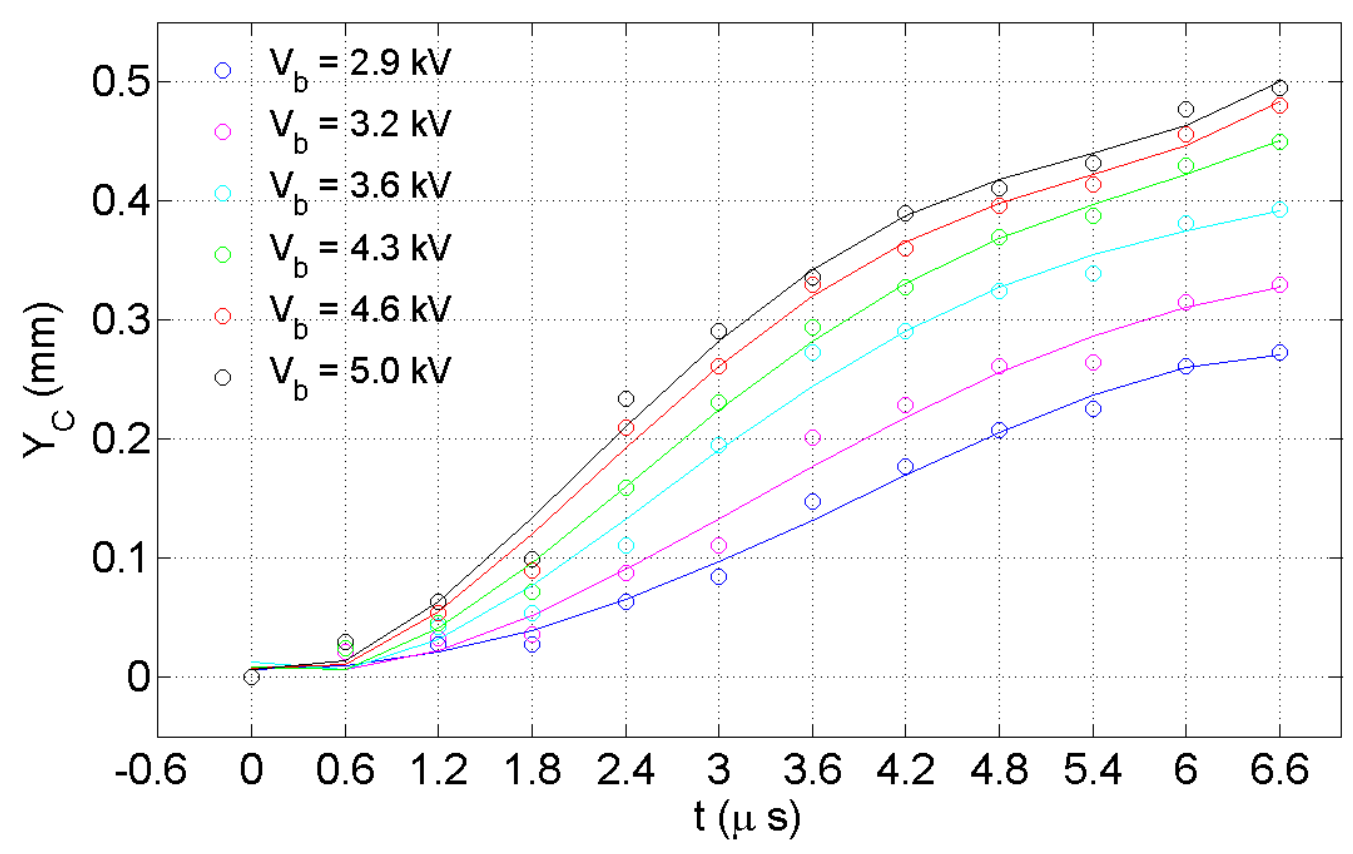

Figure 5.18: Position of the bubble centroid relative to that at the moment of shock wave impingement. Solid curves represent polynomial fits of the 4th order to the experimental data. 
Table 5.3: Migration velocity of the bubble centroid.

\begin{tabular}{cc|cc}
\hline \hline$V_{b}(\mathrm{kV})$ & $\mathrm{V}_{C}(m / s)$ & $V_{b}(\mathrm{kV})$ & $\mathrm{V}_{C}(m / s)$ \\
\hline 2.9 & 28 & 4.3 & 77 \\
3.2 & 37 & 4.6 & 87 \\
3.6 & 65 & 5.0 & 97 \\
\hline
\end{tabular}




\subsection{Experimental results: Part II}

As a second test, the case that shock wave impact occurs at a very late bubble collapse phase is considered in this section. The amplitude of the shock wave emitted from inertial cavity implosion is $P_{c}^{\prime}=9.9 \pm 1.4 \mathrm{MPa}$ and free bubble collapse time is $2 t_{c}=104.7 \pm 3.9$ (from 40 measurements). The full width at half maximum (FWHM) of the shock wave emitted from inertial cavity collapse is $\tau_{c}=51.3 \pm 12.5 \mathrm{~ns}$ (based on 15 measurements). Figure 5.19 represents a shadowgraph picture of the interaction between LSW and laser-generated single cavitation bubbles. As with the procedure discussed in section 5.6, the shock wave energy is varied in 8 steps (rows a - $\mathrm{h}$ in Figure 5.19). The shock wave pulse amplitude measured at a distance of $d \approx 1.8 \mathrm{~mm}$ above the focus location in the free field (no bubble) is between $P_{\max }=24.4 \pm 1.1 \mathrm{MPa}$ ( $a$ in Figure 5.19) and $P_{\max }=108.1 \pm 2.5$ ( $h$ in Figure 5.19).

The delay between the moment of shock wave generator trigger and observation time is $\Delta t \approx 56.6 \mu \mathrm{s}$ and kept constant for all of the image sequences (rows in Figure 5.19).

Observation starts at $t_{i}=102 \mu \mathrm{s}$ after the laser breakdown, when the LSW impinges on the cavitation bubbles (see Figure 5.7). Normalized LSW arrival time is $\alpha=t_{i} / 2 t_{c}=0.97$ (very late collapse phase). Initial bubble diameters in vertical and horizontal directions $D_{v 0}$ and $D_{h 0}$ (first frame in each row in Figure 5.19) are $0.67 \pm 0.02 \mathrm{~mm}$ and $0.64 \pm 0.01 \mathrm{~mm}$, respectively. These values are about $0.13 \mathrm{~mm}$ less than the initial bubble sizes discussed in section 5.6. Each image sequence (row), is selected out of two subsequences from several trials as explained in section 5.6. Interframe time is $0.6 \mu \mathrm{s}$ and exposure time being 20 ns. In this Figure, the laser beam comes from the right and the LSW propagates from bottom to the top. The pressure wave is recorded at $d \approx 1.8 \mathrm{~mm}$ above the focus of the LSW simultaneously with image acquisition.

By increasing the LSW energy (from $a$ to $h$ in Figure 5.19), the forced collapse happens earlier relative to the time of the shock wave impact (first frame). Comparing the Figures $5.19(\alpha=0.97)$ and $5.8(\alpha=0.91)$ it can be seen that for the same LSW amplitudes, the collapse occurs earlier in Figure 5.19. This fact illustrates the effect of initial condition of the bubble on its forced collapse. For a certain amount of shock wave energy, comparing two cases $(\alpha=0.91$ and $\alpha=0.97$ ), in the latter condition, the bubble has less chance to be affected by the negative part of the LSW profile. Therefore, the collapse is less retarded and a more violent bubble implosion is expected with resultant of emission of shock waves with larger amplitudes.

Amplification of the amplitude of the shock wave emitted from the forced cavity collapse compared with inertial bubble oscillation, is considered in 5.7.1. The effect of reduction of bubble collapse time is discussed in 5.7.2. In subsection 5.7.3 the pulse amplitude of the lithotripter shock wave (LSW) after passing through the laser-generated single cavitation bubbles is analyzed and compared with previous results. The velocity of the shock wave-induced liquid jet as well as the displacement of the bubble centroid are investigated in 5.7.4. 


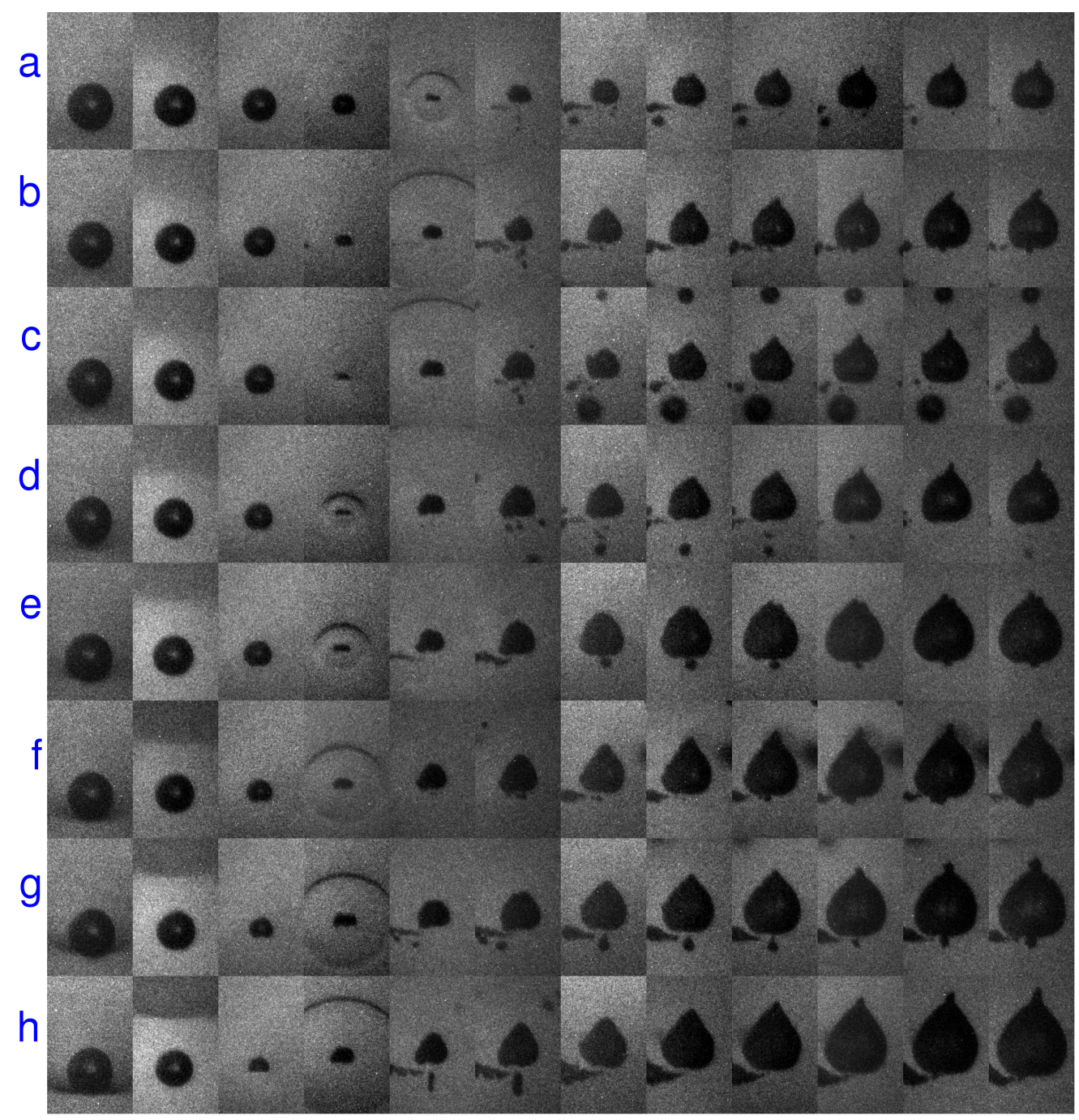

Figure 5.19: High-speed image sequences of interaction between lithotripter shock wave and laser-induced single cavitation bubbles. Normalized LSW arrival time is $\alpha=0.97$. Interframe time is $0.6 \mu \mathrm{s}$ and exposure time is $20 \mathrm{~ns}$. Frame width and height are $1.2 \mathrm{~mm}$ and $1.9 \mathrm{~mm}$, respectively. The shock wave propagates from bottom to the top and impinges on the cavity on the first frame in each row. The shock strength is increasing from $a$ to $h$ and is kept constant for every image sequence (row). The shock wave positive pulse amplitudes measured at the distance of $d \approx 1.8 \mathrm{~mm}$ above the shock focus in the free field (no bubble) are $P_{\max }=$ 24.4(a), 29.9(b), 37.1(c), 45.9(d), 61.9(e), 79.1(f), 95.8(g), 108.1(h) MPa. Initial bubble diameter in vertical direction is $0.67 \pm 0.02 \mathrm{~mm}$ and the initial horizontal cavity diameter is $0.64 \pm 0.01 \mathrm{~mm}$. The laser beam enters from the right. 
Typical acoustic wave profiles measured at a distance of $d \approx 1.8 \mathrm{~mm}$ above the shock wave focus location are illustrated in Figure 5.20. The graphs correspond to three intensity levels of the shock wave source. Signals are calibrated and deconvoluted by the response function of the fiber optic probe hydrophone (FOPH). In this Figure, the moment of the cavitation bubble implosion shifts to the left with increasing the LSW strength.
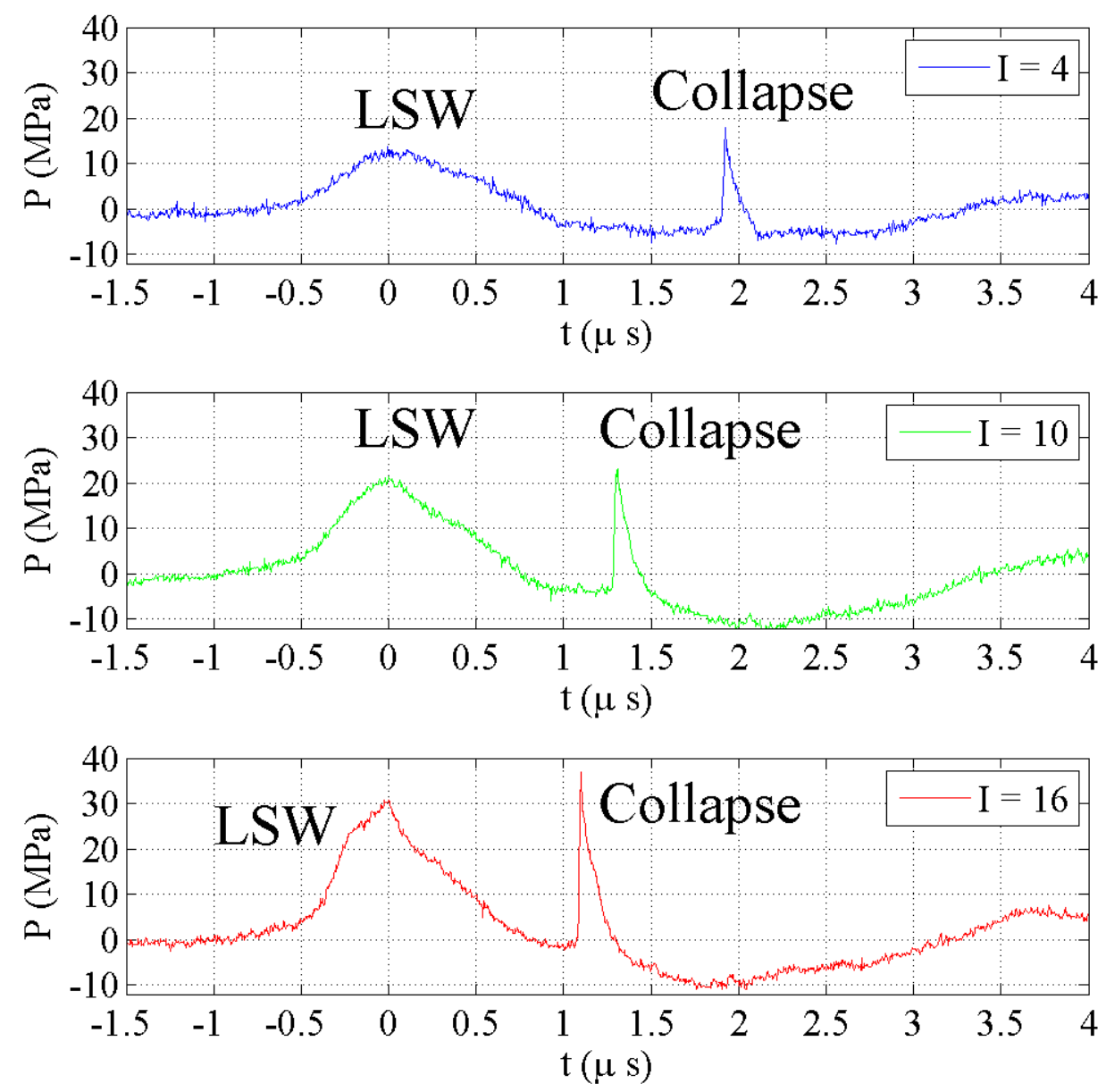

Figure 5.20: Pressure wave profile above the laser-generated single cavitation bubbles exposed to a lithotripter shock wave. Bubble collapse happens earlier by increasing the LSW intensity. Note that for each specific intensity level $I$, the collapse takes place earlier compared with the corresponding case displayed in Figure 5.9. 


\subsubsection{Pressure enhancement}

The effect of amplification of the shock wave emitted from bubble collapses under the influence of LSWs is illustrated in Figure 5.21. For different LSW energies, the ratio between the forced and free collapse pressure amplitudes $R=P_{c} / P_{c}^{\prime}\left(P_{c}\right.$ is the pulse amplitude of the shock wave emitted from the forced bubble collapse and $P_{c}^{\prime}=9.9 \pm 1.4 \mathrm{MPa}$ ) is plotted. Errors in measurement of $P_{c}$ are dominated by the bias noise level of the hydrophone signal which is $\approx 0.65 \mathrm{MPa}$.

It can be seen that the shock wave amplitude from the forced bubble collapse $P_{c}$ is increasing with the LSW intensity. The pressure amplification factor $R$ is between $\approx 2.2$ and $\approx 4.1$. These values are larger than the ones discussed in section 5.6.1 $(\alpha=0.91,1.6<R<2.7)$, which indicates that the bubble has collapsed more violently and stronger shock waves are emitted compared with the case discussed in section 5.6.1. For peak voltages of the shock wave source $V_{b}$ larger than $4.0 \mathrm{kV}$, the time interval between moments of the LSW arrival and bubble forced collapse nearly matches the duration of the compression phase of the LSW profile. In these cases, maximum energy is transferred from the shock wave to the cavity interior and the most violent bubble implosions take place.

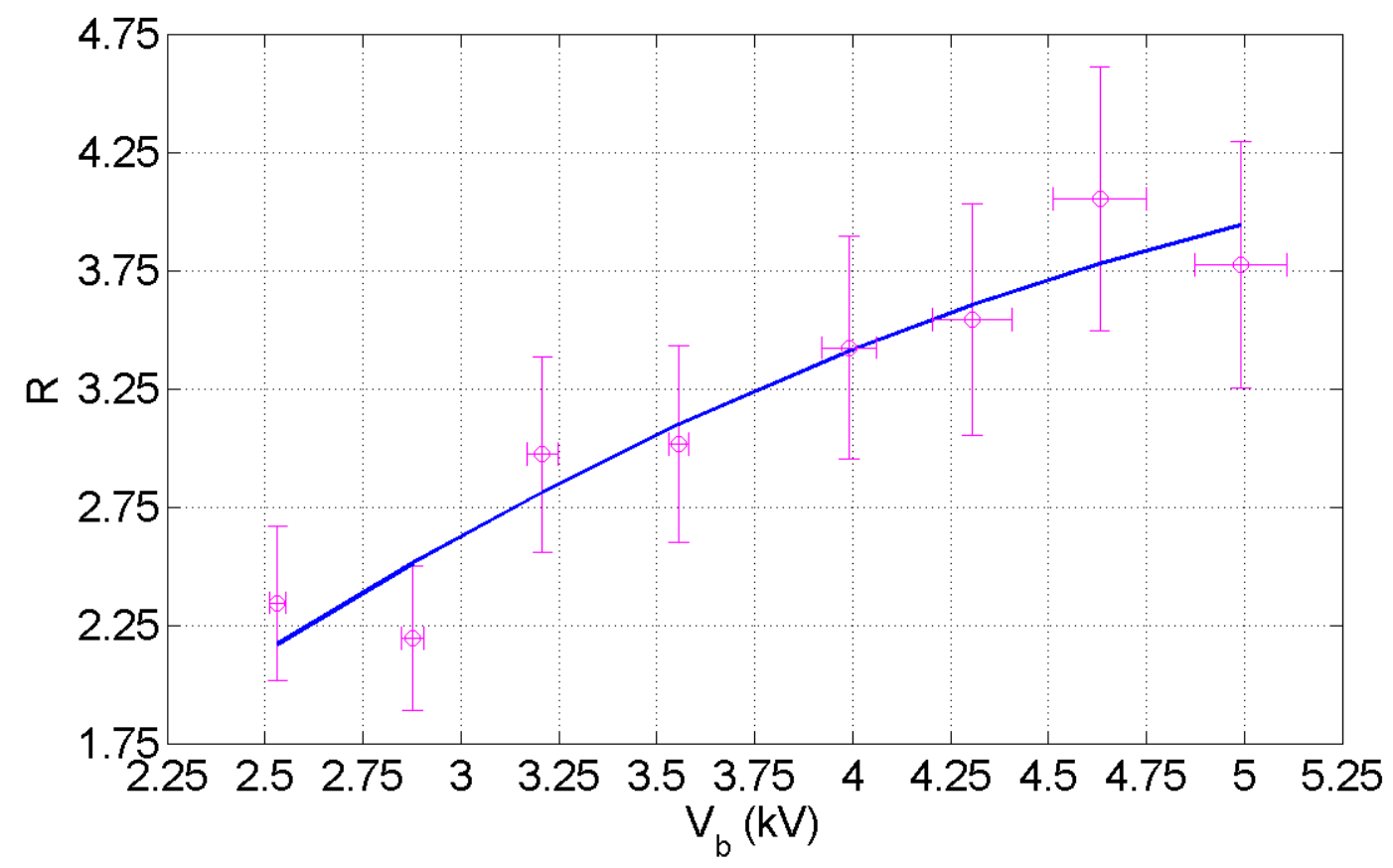

Figure 5.21: Amplification of the collapse shock wave due to the application of the lithotripter shock wave (LSW). Error bars show the bias noise level of the FOPH signal. The solid line illustrates a quadratic fit to the experimental results. 


\subsubsection{Reduction of collapse time}

The time interval between lithotripter shock wave impingement on single cavities and forced bubble collapse $\Delta t_{i f}$, is plotted in Figure 5.22 for application of different LSW intensities. This parameter is shortened by increasing the shock wave intensity or peak voltage of the shock wave source. The variable $\Delta t_{\text {if }}$ is reduced rapidly for voltages in the range $V_{b}<\approx 4.0 \mathrm{kV}$. Nevertheless, for $V_{b}$ larger than $\approx 4.0 \mathrm{kV}$, truncation of implosion time behaves almost smoothly.

Maximum reduction in collapse time (minimum of parameter $\Delta t_{i f}$ ) occurs in the case of applying the intensity level $I=18\left(V_{b}=5.0 \mathrm{kV}\right)$, which corresponds to $\Delta t_{i f} \approx 1.1 \mu \mathrm{s}$. This means that the bubble collapse occurs at $\approx 1.1 \mu \mathrm{s}$ after the LSW impact. Therefore, most part of the negative tail of the LSW profile arrives after bubble collapse and it can not have any influence on the first oscillation cycle of bubble dynamics. An intense cavity implosion occurs in this case. The parameter $\Delta t_{i f}$ attains values between $1.1 \mu \mathrm{s}$ and $1.9 \mu \mathrm{s}$. These values are less than the ones derived for the case $\alpha=0.91$, which are in the range of $1.9 \mu \mathrm{s}<\Delta t_{i f}<3.2 \mu \mathrm{s}$ (section 5.6.2). Then, the amount of reduction of $\Delta t_{i f}$ is sensitive to the shock wave arrival time. The collapse time is more reduced for the case $\alpha=0.97$ (this section) compared with $\alpha=0.91$ (section $5.6 .2)$.

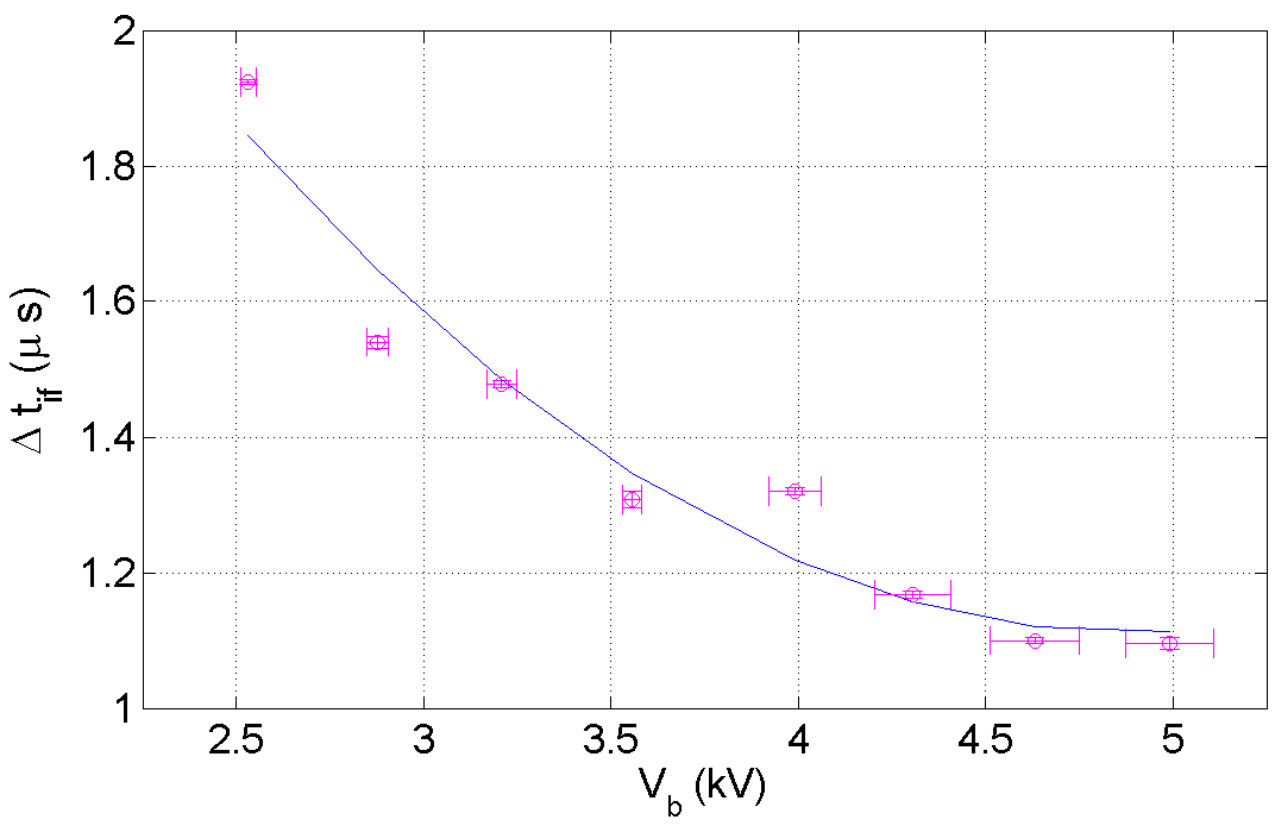

Figure 5.22: Reduction of bubble collapse time due to interaction between LSW and laser-generated single bubbles. Parameter $\Delta t_{i f}$ is the time interval between lithotripter shock wave impact on the bubble and the forced bubble collapse. Error bars are standard deviation of the experimental data. The solid line is related to a quadratic fit to the experimental results. 


\subsubsection{Lithotripter shock wave (LSW) pulse amplitude}

The LSW pulse amplitude after passage through the laser-generated single cavitation bubble is illustrated in Figure 5.23. Ignoring the case $V_{b} \approx 5.0 \mathrm{kV}$, it seems to be an almost linear relation between the LSW peak pressure and the shock wave source maximum voltage $V_{b}$ (similar to the results in 5.6.3). Equation of the fitted line to the experimental data is $P_{L S W}(\mathrm{MPa})=(9.10 \pm$ $1.82) V_{b}(\mathrm{kV})-8.90 \pm 6.66$. By increasing the peak voltage $V_{b}$ from $\approx 2.5 \mathrm{kV}$ to $\approx 4.6 \mathrm{kV}$, the pulse amplitude of the shock wave above the bubble $P_{L S W}$ rises from $\approx 13.9 \mathrm{MPa}$ to $\approx 30.8 \mathrm{MPa}$.

The parameter $R_{P}$ is defined as the ratio between the lithotripter shock wave peak pressures for the present results $(\alpha=0.97)$ and the ones discussed in section 5.6.3 $(\alpha=0.91)$. It is plotted in Figure 5.24 for several shock wave energies. As the difference in the size of cavitation bubbles at the moment of shock wave impact for these two cases is small (i.e. $\approx 0.13 \mathrm{~mm}$ ), the variable $R_{P}$ is expected to be almost one or slightly larger than that and nearly independent on the shock wave intensity.

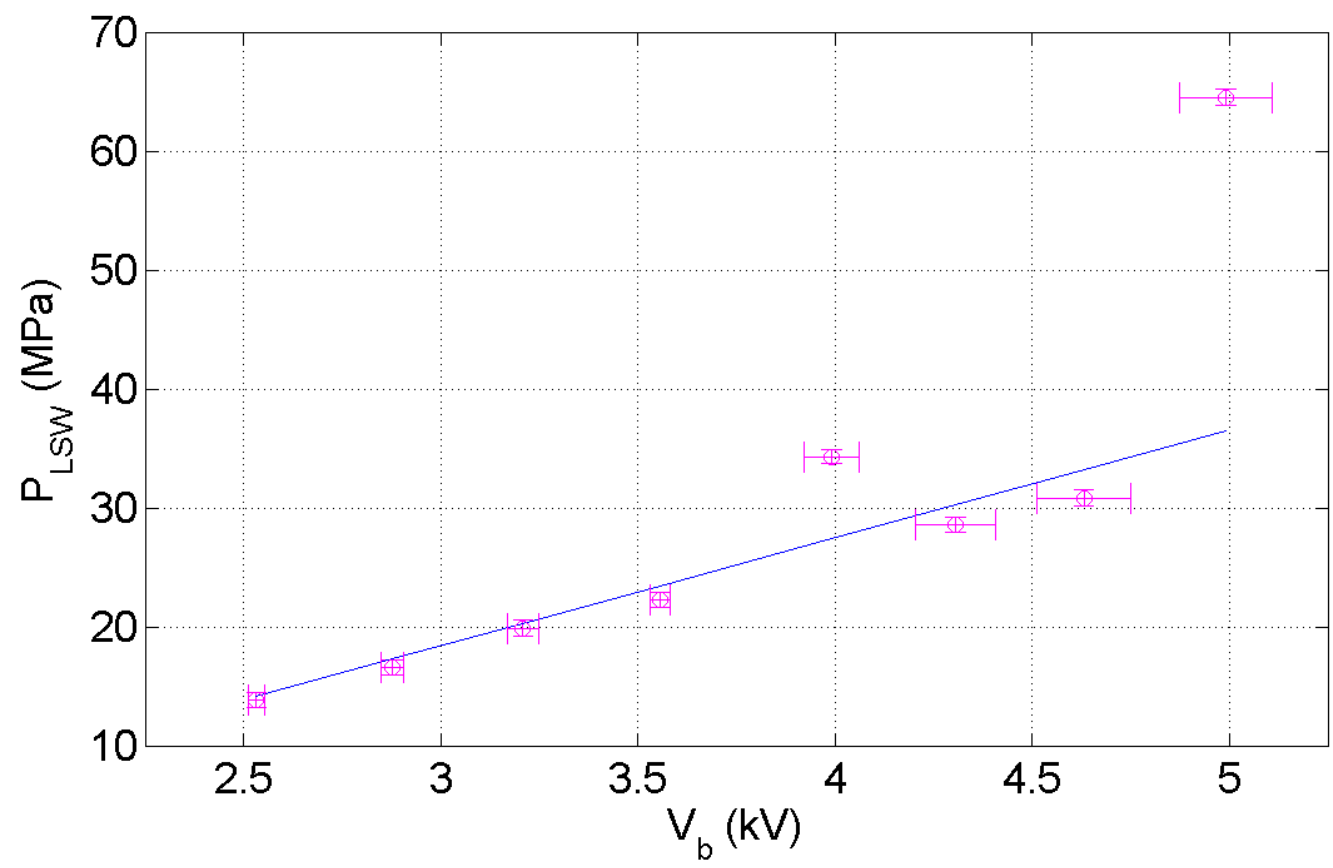

Figure 5.23: Lithotripter shock wave peak pressure in the presence of lasergenerated single cavitation bubbles. The solid line represents a linear fit to the experimental results (except the last data point). 


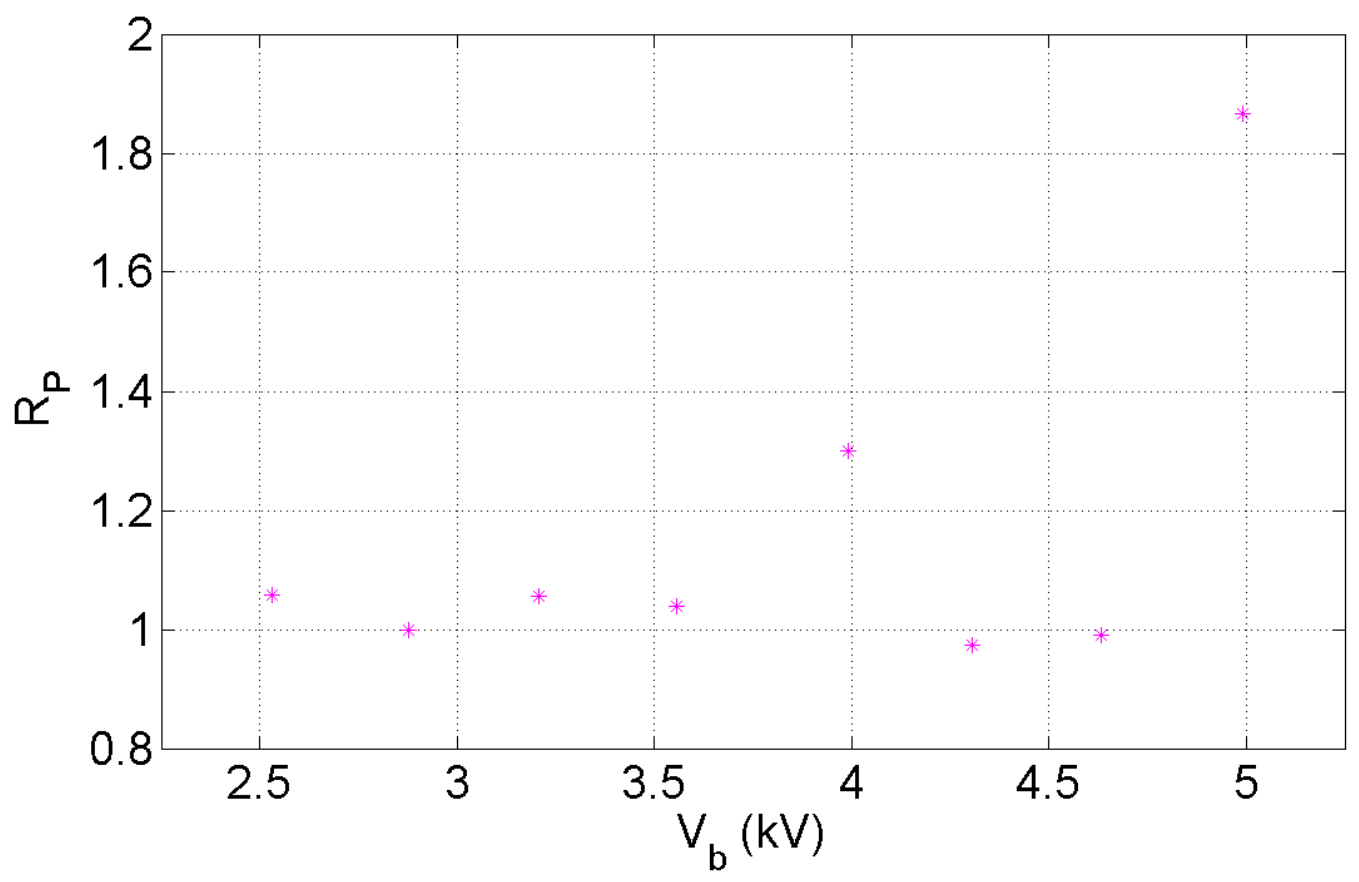

Figure 5.24: Comparison of the LSW pulse amplitudes for two shock wave arrival times. $R_{P}=P_{L S W}(\alpha=0.97) / P_{L S W}(\alpha=0.91)$.

\subsubsection{Bubble dynamics under the influence of the applied lithotripter shock wave (LSW)}

Asymmetric collapse of cavitation bubbles in response to shock wave impingement is illustrated in Figure 5.25. Parameters $R_{1}$ and $R_{2}$ are defined as the ratio between bubble diameters in vertical and horizontal directions $D_{v}$ and $D_{h}$, and their values at the moment of shock wave impact $D_{v 0}$ and $D_{h 0}\left(R_{1}=D_{v} / D_{v 0}\right.$ and $\left.R_{2}=D_{h} / D_{h 0}\right)$. This time coincides with the moment of zero in Figure 5.25. The experimental results are obtained at two intensity levels of the shock wave generator $\left(V_{b} \approx 2.9 \mathrm{kV}, I=6\right.$ and $\left.V_{b} \approx 4.6 \mathrm{kV}, I=16\right)$.

The case $V_{b} \approx 2.9 \mathrm{kV}$ leads to a variation of the dimensionless parameter $R_{1}$ by rate of $\approx 0.29 / \mu \mathrm{s}$, in the time interval between bubble collapse and $\Delta t=$ $3.0 \mu$ s later. For horizontal bubble diameter, the temporal rate of variation of $R_{2}$ is $\approx 0.19 / \mu$ s (see Figure 5.25 (top)). Considering the same time interval and using the intensity level $I=16$ of the shock wave source (see Figure 5.25 (bottom)), the temporal variation for the defined parameters $R_{1}$ and $R_{2}$ are $\approx 0.37 / \mu$ s and $\approx 0.24 / \mu \mathrm{s}$, respectively.

The temporal variation of vertical dimensionless bubble diameter is about 1.5 times faster than that of horizontal orientation (similar to the case shown in section 5.6.4). This is the result of shock wave impact which breaks the spherical symmetry of the bubble collapse. 

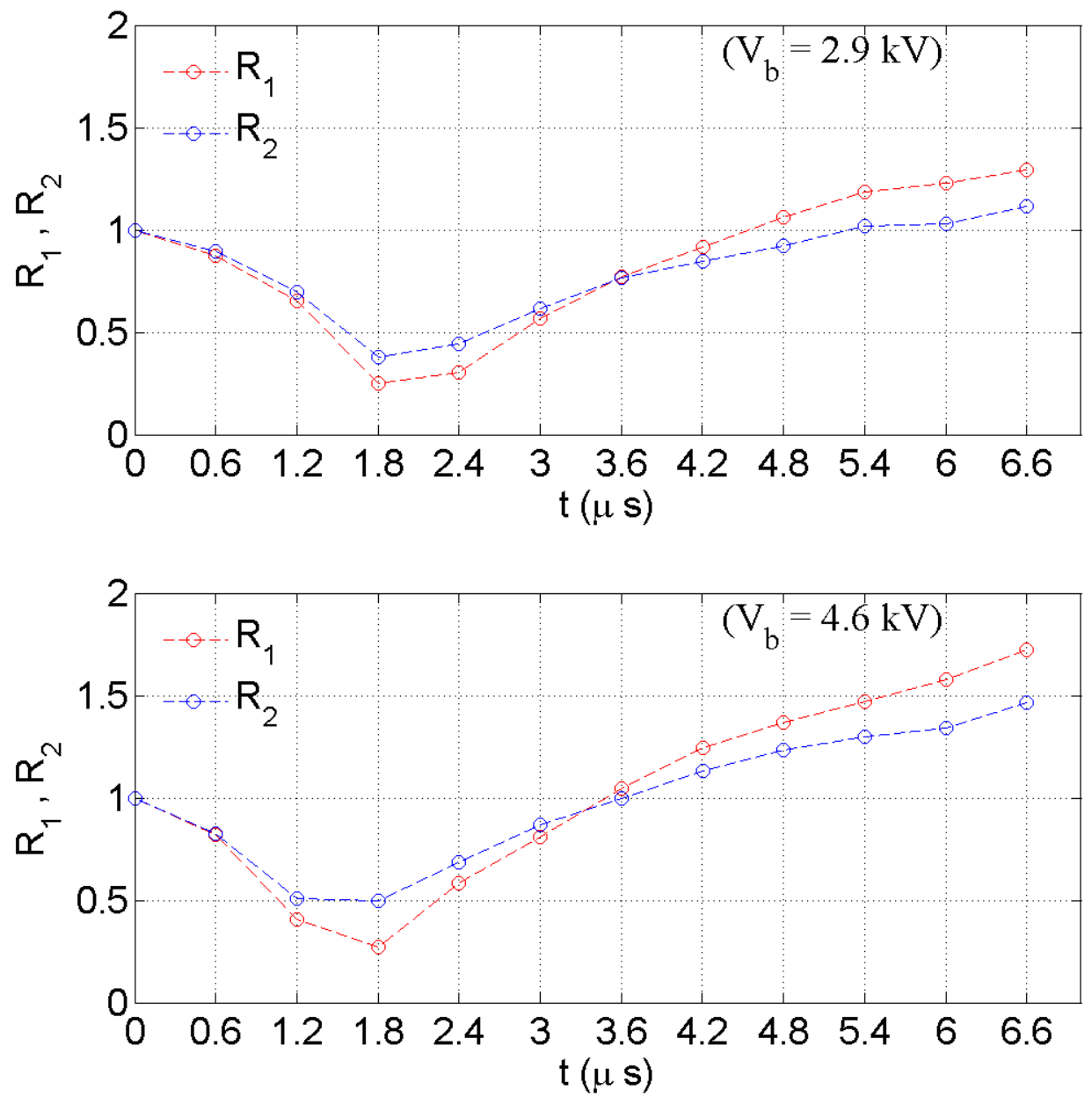

Figure 5.25: Temporal variation of dimensionless vertical and horizontal diameters of laser-generated single cavitation bubbles. The lithotripter shock wave impinges on the bubbles at time $t=0$.

Figure 5.26 illustrates the distance of lower and upper bubble walls from the top of each frame in Figure 5.19, after the impingement of the LSW $(t=0)$. The applied shock wave source peak voltages are $V_{b} \approx 2.9 \mathrm{kV}$ and $V_{b} \approx 5.0 \mathrm{kV}$.

Maximum jet velocities are calculated based on selection of the pair of points explained in section 5.6 .4 (averaged over $0.6 \mu \mathrm{s}$ ). They are $V_{j} \approx 510 \mathrm{~m} / \mathrm{s}$ and $V_{j} \approx 580 \mathrm{~m} / \mathrm{s}$ for $V_{b} \approx 2.9 \mathrm{kV}(I=6)$ and $V_{b} \approx 5.0 \mathrm{kV}(I=18)$, respectively. For the case $I=6$, the maximum jet velocity derived here $(\alpha=0.97)$ is by a factor of $\approx 1.3$ times larger than the one for $\alpha=0.91$, which is $V_{j} \approx 380 \mathrm{~m} / \mathrm{s}$. 

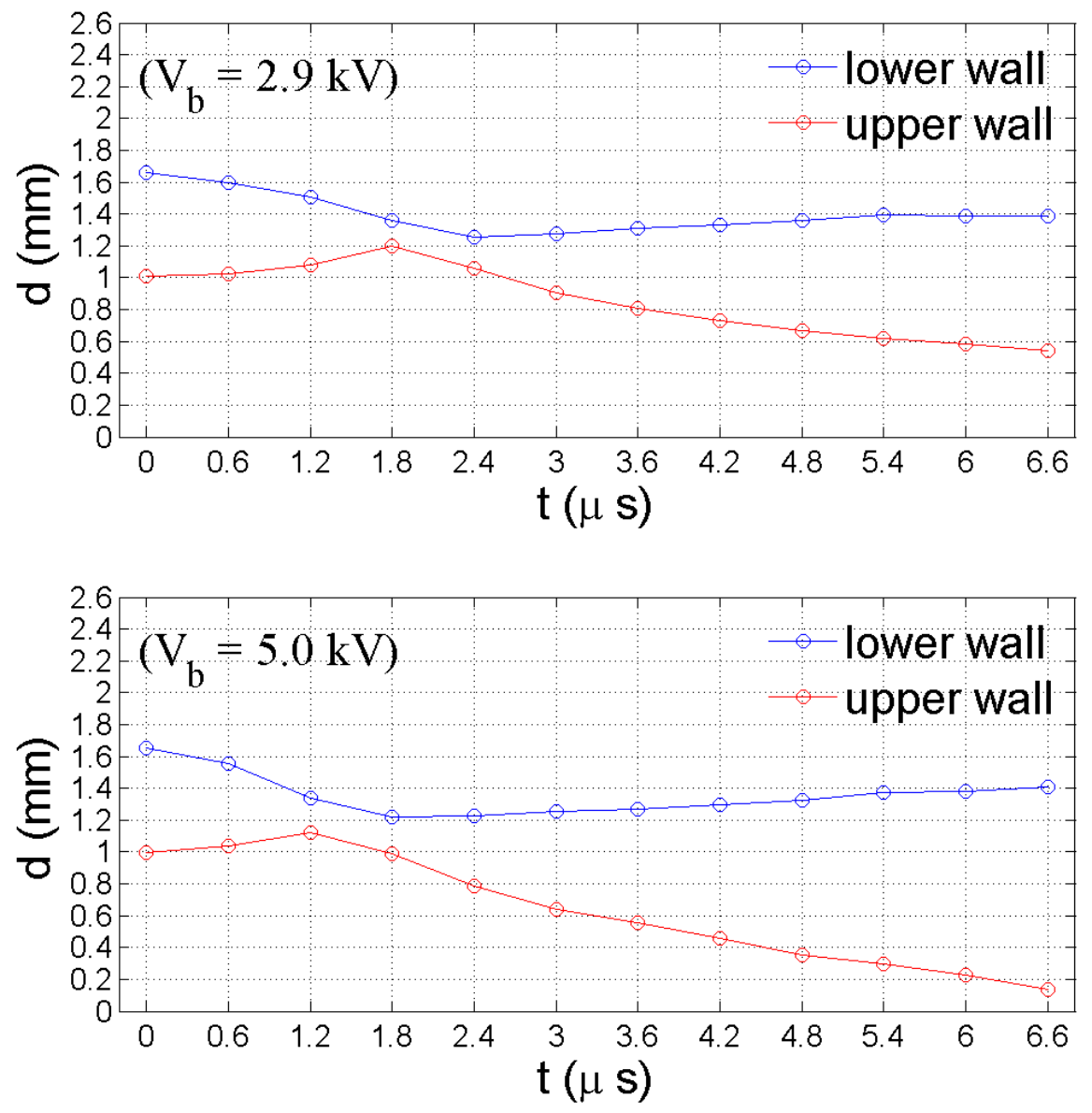

Figure 5.26: Temporal variation of distance $(d)$ of the lower and upper bubble walls from the top of each frame in Figure 5.19.

The second method for estimating the jet speed, as considered in section 5.6.4, is based on the temporal variation of the bubble tip location. The spatial position of the bubble tip $Y_{t}$ relative to that at the moment of collapse $\left(Y_{t}(0)=\right.$ $0)$ is plotted in Figure 5.27. The zero in time indicates the moment of cavity implosion. The tip velocities (averaged over a time interval of $3 \mu \mathrm{s}$ ) are $V_{t} \approx$ $146 \mathrm{~m} / \mathrm{s}$ and $V_{t} \approx 212 \mathrm{~m} / \mathrm{s}$ for $V_{b} \approx 2.9 \mathrm{kV}$ and $V_{b} \approx 5.0 \mathrm{kV}$, respectively.

Similar to the results in section 5.6.4, the velocities derived by this method are far below the ones calculated from the first procedure, which was based on the averaging over $0.6 \mu \mathrm{s}$. The bubble tip in the case $V_{b}=5.0 \mathrm{kV}$ jumps faster than that for $V_{b}=2.9 \mathrm{kV}$. This fact indicates that by increasing the LSW amplitude, the liquid jet initiates more strongly and a larger amount of energy is transferred from the lithotripter shock wave to the cavity interior. For the case $V_{b}=2.9 \mathrm{kV}$, the bubble tip travels faster in the condition of $\alpha=0.97$ by a factor of $\approx 1.6$ times that in the case of the dimensionless shock wave arrival time $\alpha=0.91$. 


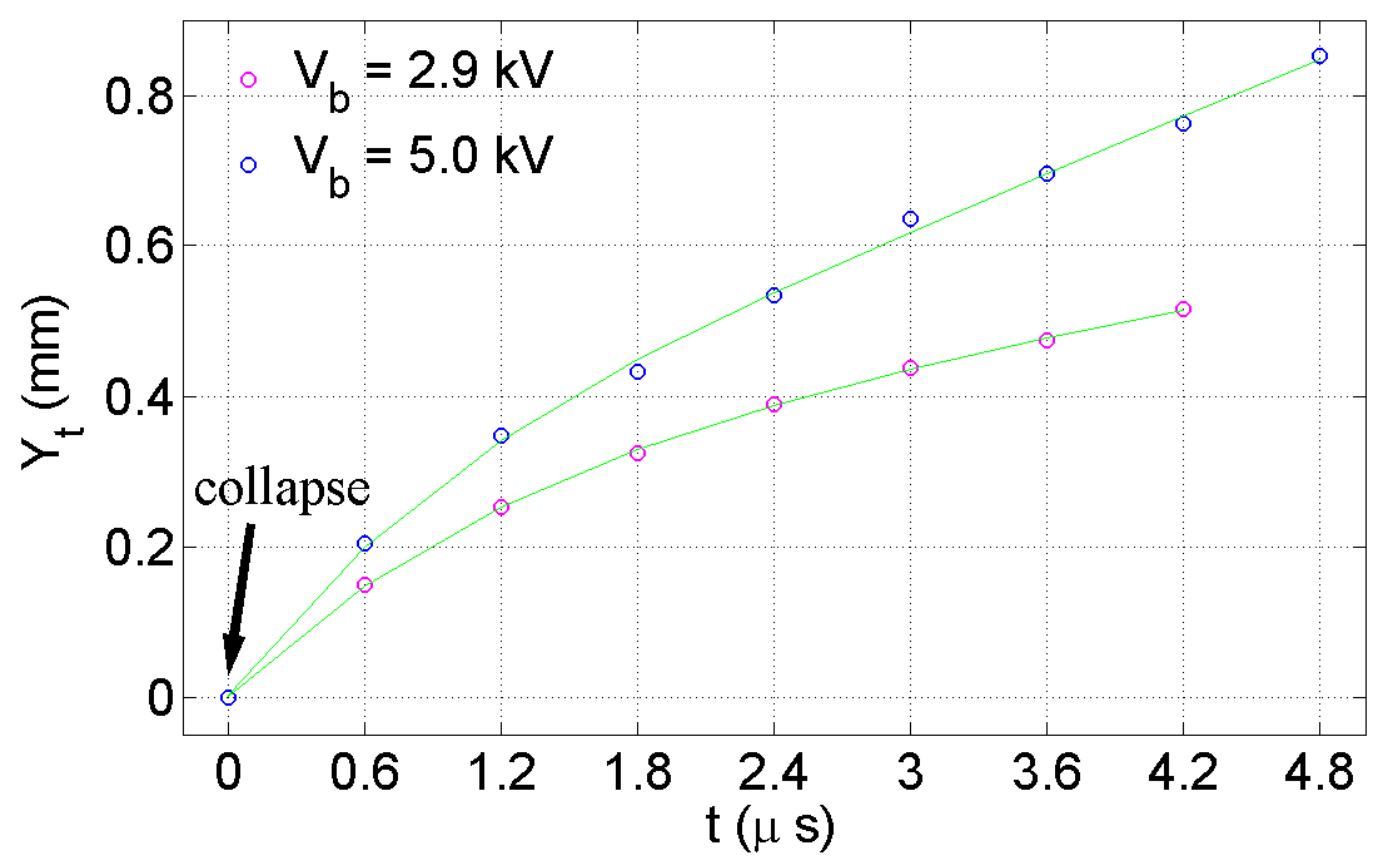

Figure 5.27: Relative position of the bubble tip from the time of collapse. Time of zero coincides with bubble collapse. The solid lines represent polynomial fits of the 4th order to the experimental data points. Deviation of the two plots is rising by time.

The spatial position of the bubble centroid $Y_{C}$ relative to its initial value $\left(Y_{C}=0\right.$ at $\left.t=0\right)$ is plotted in Figure 5.28. The moment of $t=0$ indicates the time of LSW impact. It is visible that the bubble is migrated in the direction of shock wave propagation (from bottom to top in Figure 5.19). Figure 5.28 illustrates the experimental results for using of 4 intensity levels of the shock wave generator (the peak voltage of the shock wave generator ranges from $V_{b} \approx 3.2 \mathrm{kV}$ to $\left.V_{b} \approx 5.0 \mathrm{kV}\right)$.

Despite the case considered in section $5.6(\alpha=0.91)$, even before $t=0.6 \mu \mathrm{s}$ from the shock impingement, a slight displacement of the cavity centroid is visible in this condition $(\alpha=0.97)$. In the time interval of $t=0.6 \mu$ s to $t=3.6 \mu \mathrm{s}$, there is a rapid migration of the cavity centroid and then, it begins to damp out.

Bubble centroid velocities $V_{C}$ averaged over $3.0 \mu$ s are shown in table 5.4. These values are larger than their corresponding ones explained in section 5.6.4, which indicates that the bubble centroid is translated more if the shock wave impinges in a very late stage of the bubble collapse phase. 


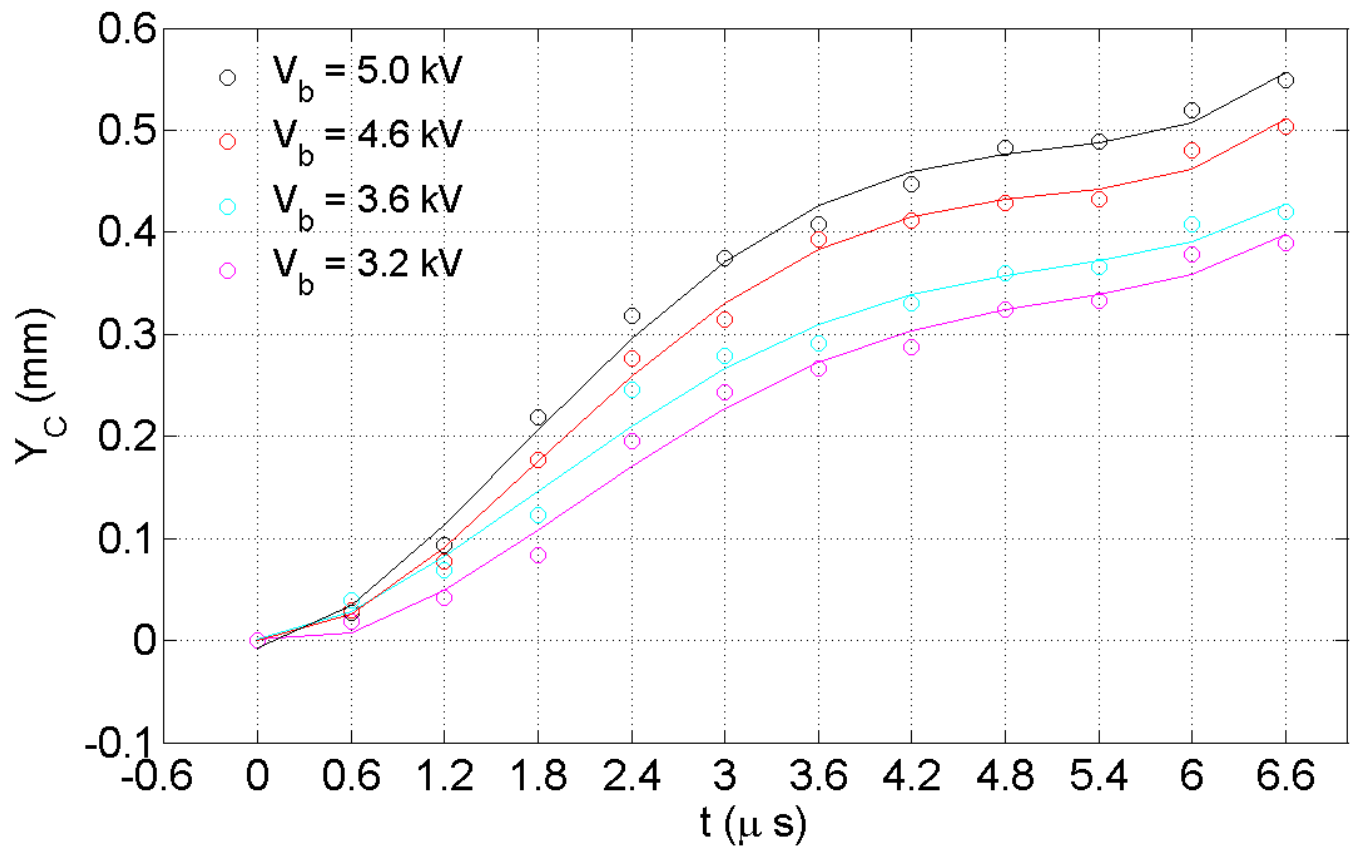

Figure 5.28: Displacement of the centroid of laser-generated single cavitation bubbles exposed to lithotripter shock waves. The solid lines are polynomial fits of the 4th order to the experimental results.

Table 5.4: Migration velocity of the bubble centroid.

\begin{tabular}{cc}
\hline \hline$V_{b}(\mathrm{kV})$ & $V_{C}(\mathrm{~m} / \mathrm{s})$ \\
\hline 3.2 & 81 \\
3.6 & 93 \\
4.6 & 105 \\
5.0 & 125 \\
\hline
\end{tabular}




\subsection{Experimental results: Part III}

In this part, the case that the lithotripter shock wave impacts on laser-generated single cavitation bubbles at the moment of $t=12 \mu$ s after optical breakdown is investigated. At this time, single bubbles are in the expansion phase and have a similar size to the case considered in section $5.6(\alpha=0.91)$. This fact allows to study the effect of bubble oscillation phase on shock wave - bubble interaction.

Shock waves emitted from free inertial collapse of single cavities, have pulse amplitude of $P_{c}^{\prime}=7.9 \pm 1.0 \mathrm{MPa}$, and the collapse time of the bubbles in the free field is $2 t_{c}=101.9 \pm 3.0 \mu \mathrm{s}$ (based on 40 measurements). Full width at half maximum (FWHM) of free collapse shock is $\tau_{c}=49.3 \pm 12.2 \mathrm{~ns}$ (from 15 measurements).

High-speed shadowgraph image sequences revealing the interaction between lithotripter shock waves and laser-generated single cavitation bubbles are given in Figure 5.29. Vertical and horizontal bubble diameters at the moment of LSW impingement are $D_{v 0}=0.82 \pm 0.02 \mathrm{~mm}$ and $D_{h 0}=0.78 \pm 0.01 \mathrm{~mm}$, respectively. Observation starts at the moment of shock wave impact (i.e. at $t_{i}=12 \mu \mathrm{s}$ after laser breakdown). Then, normalized shock wave impingement time is $\alpha=t_{i} / 2 t_{c}=0.12$, which indicates that the bubble is in its early expansion phase at the moment of the shock wave exposure.

The delay between the shock wave source trigger and observation remains constant for all the image series. The laser beam enters from right and the LSW propagates from bottom to top. The interframe and exposure times are $0.6 \mu \mathrm{s}$ and $20 \mathrm{~ns}$, respectively. Intensity level $(I)$ of the LSW source is varied in 8 steps $(I=4,6, \ldots 18)$. The values of LSW pressure pulse amplitude related to several intensity levels of the shock wave generator in the free field, are tabulated in table 5.1.

In the same manner as discussed in sections 5.6 and 5.7, the collapse occurs earlier by increasing the LSW intensity (from $a$ to $h$ in Figure 5.29). If we compare Figures 5.8 and 5.29, it can be seen that with the same initial bubble size and LSW energy, the collapse occurs later for the case the bubble is in the expansion phase at the time of shock wave arrival (Figure 5.29). This is due to the fact that when the shock arrives, it first stops the succeeding expansion of the bubble and then forces it to collapse. In fact, a work is done against the outward bubble wall velocity. This procedure needs more time in comparison to the case when the initial bubble is already collapsing.

Enhancement of the collapse shock wave amplitude and reduction of implosion time compared with the inertial cavity oscillation, are considered in sections 5.8.1 and 5.8.2, respectively. It will be shown in the following sections that forced collapse shock amplitude is weak compared to the case of initial bubbles being in the collapse phase with the same size. In section 5.8.3 the pulse amplitude of the lithotripter shock wave (LSW) after passing through the laser-generated single cavitation bubbles is analyzed. 


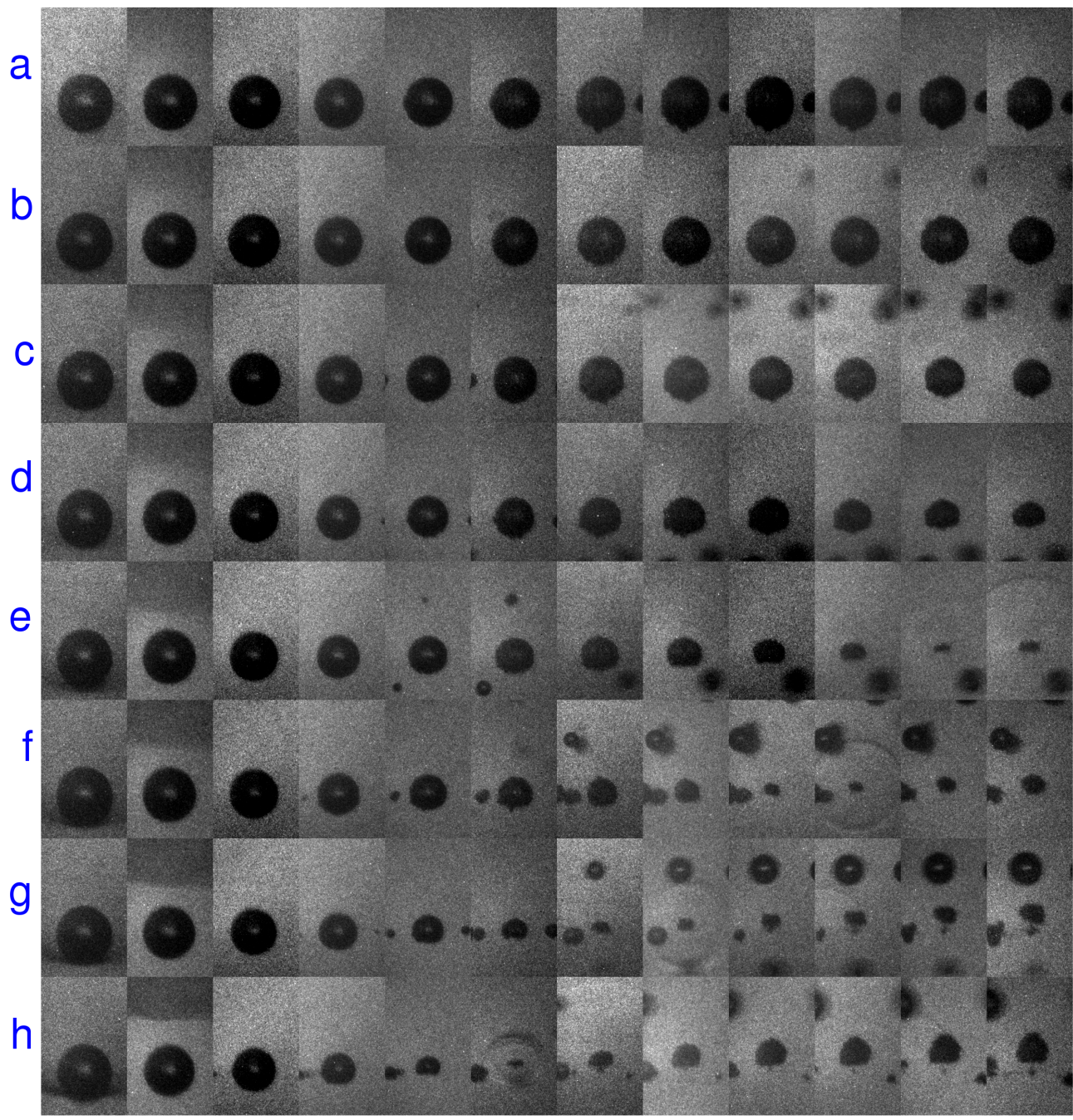

Figure 5.29: High-speed image sequences of interaction between lithotripter shock wave and laser-induced single cavitation bubbles. Normalized LSW arrival time is $\alpha=0.12$. Interframe time is $0.6 \mu \mathrm{s}$ and exposure time being 20 ns. Frame width and height are $1.2 \mathrm{~mm}$ and $1.9 \mathrm{~mm}$, respectively. The shock wave propagates from bottom to the top and impinges on the cavity on the first frame in each row. The shock strength is increasing from $a$ to $h$ and is kept constant for every image sequence (row). The peak pressures of the shock wave measured at the distance of $d \approx 1.8 \mathrm{~mm}$ above the shock focus location in the free field (no bubble) are $P_{\max }=$ 24.4(a), 29.9(b), 37.1(c), 45.9(d), 61.9(e), 79.1(f), 95.8(g), 108.1 $(h) \mathrm{MPa}$. Initial bubble diameter in vertical direction is $D_{v 0}=0.82 \pm 0.02 \mathrm{~mm}$ and its initial horizontal diameter is $D_{h 0}=0.78 \pm 0.01 \mathrm{~mm}$. The laser beam enters from right. 


\subsubsection{Pressure enhancement}

The time history of the acoustic field is recorded at a distance of $d \approx 1.8 \mathrm{~mm}$ above the LSW focus simultaneously with the image acquisition. Figure 5.30 depicts the enhancement of shock waves emitted from bubble collapse under the influence of the applied lithotripter shock wave. The ratio between shock wave amplitude of forced and inertial cavity collapse $R=P_{c} / P_{c}^{\prime}$ is plotted. The results correspond to the case of the peak voltage of the shock wave source being in the range $V_{b} \geq \approx 4.0 \mathrm{kV}$. It has to be mentioned that at lower LSW energies, the collapse shock wave amplitude is so weak that it could not be distinguished clearly. Errors are mostly related to the bias noise level of the fiber optic probe hydrophone (FOPH) signal.

Comparing the results with the case considered in section 5.6.1 $(\alpha=0.91)$, it can be concluded that the collapse pressure is more enhanced if the initial bubble is at the collapse phase (as in section 5.6.1). For example, at voltage $V_{b} \approx 5.0 \mathrm{kV}(I=18)$, and similar initial bubble size (the bubble diameter is $D \approx 0.8 \mathrm{~mm}$ ), the pressure amplification factor for the case that the initial bubble is in the collapse phase is $R=2.70 \pm 0.39$. But this value for the initially expanding cavity is $R=1.19 \pm 0.18$. In general, the values shown in this plot, are well below the ones shown in Figure 5.10, for the same intensity levels of the shock wave source. This fact indicates the effect of oscillation phase on shock wave pressure enhancement. The initial expanding bubbles lead to less violent collapses resulting in weaker shock wave emissions.

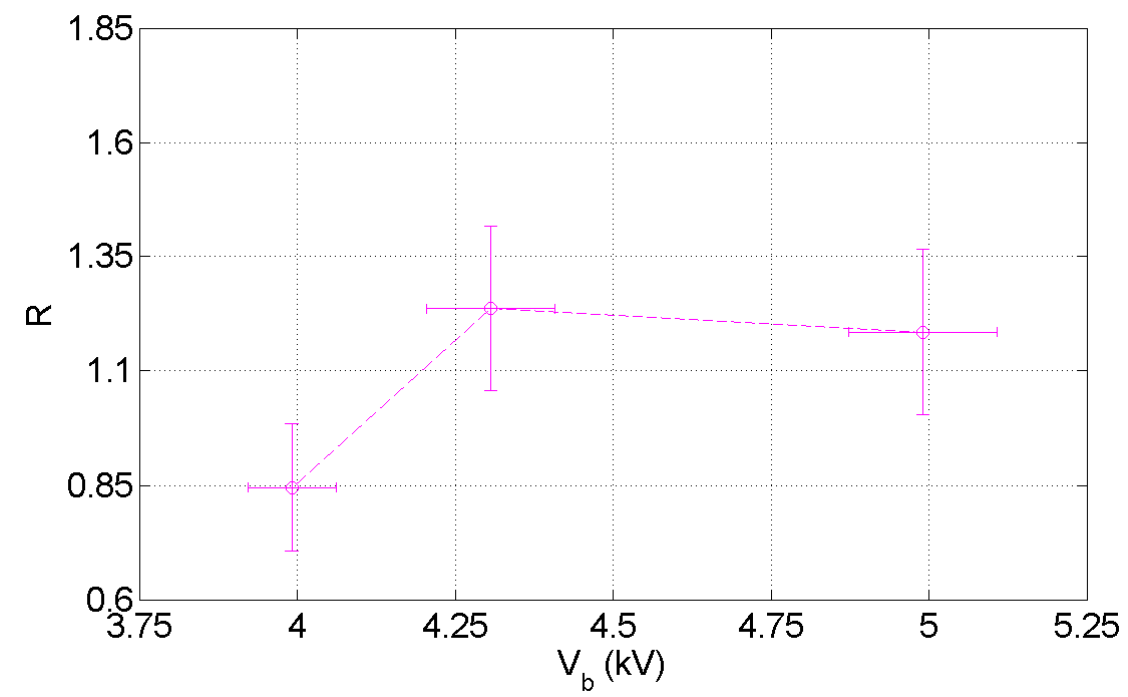

Figure 5.30: Enhancement of the shock wave peak pressure released from bubble collapse due to the application of lithotripter shock waves. The error bars are calculated from the standard deviation of experimental data. The dashed line connects the data points to guide the eye. Normalized LSW arrival time is $\alpha=0.12$. 


\subsubsection{Reduction of collapse time}

Figure 5.31 represents the time interval between lithotripter shock wave impingement on single cavitation bubbles and moment of forced bubble collapse, $\Delta t_{i f}$ as a function of peak voltage of the shock wave generator. There is a nearly linear relation for decreasing of parameter $\Delta t_{i f}$. The equation of fitted line to the experimental data is $\Delta t_{i f}(\mu \mathrm{s})=(-3.11 \pm 0.03) V_{b}(\mathrm{kV})+18.30 \pm 0.15$.

The effect of bubble oscillation phase on truncation of its collapse time is illustrated in table 5.5. The time interval $\Delta t_{i f}$ in the case that the initial bubble being at its early expansion phase $(\alpha=0.12)$ is by a factor of $1.5-2.7$ times that if the initial bubble is in late collapse phase (section 5.6.2, $\alpha=0.91$ ). The weakness of the pressure signal for $V_{b}$ less than $4.0 \mathrm{kV}$ does not allow a comparison of the parameter $\Delta t_{i f}$ in the full range of the applied shock wave intensities.

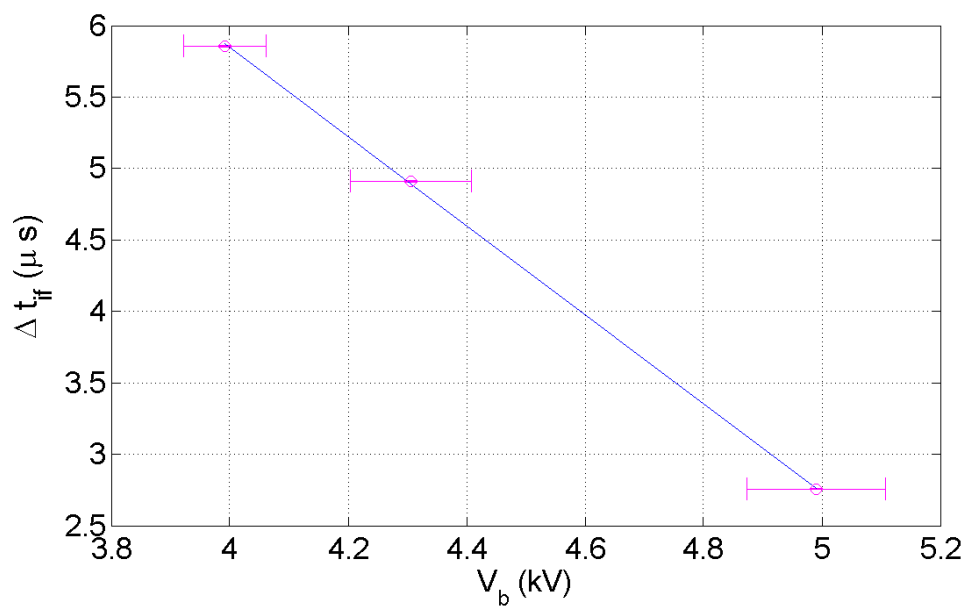

Figure 5.31: Time interval between the lithotripter shock wave (LSW) impingement on the laser-generated single bubble and the forced bubble collapse. The solid line represents a linear fit to the experimental data. Normalized LSW arrival time is $\alpha=0.12$.

Table 5.5: Time interval between LSW impact and forced bubble collapse.

\begin{tabular}{ccc}
\hline \hline$V_{b}(\mathrm{kV})$ & $\Delta t_{i f}(\mu s)(\alpha=0.12)$ & $\Delta t_{i f}(\mu s)(\alpha=0.91)$ \\
\hline 4.0 & 5.86 & 2.16 \\
4.3 & 4.91 & 2.13 \\
5.0 & 2.76 & 1.86 \\
\hline
\end{tabular}




\subsubsection{Lithotripter shock wave (LSW) pulse amplitude}

The pulse amplitude of LSW measured at distance of $d \approx 1.8 \mathrm{~mm}$ above its focus is plotted in Figure 5.32 for using three intensity levels of the shock wave generator. The LSW peak pressure ranges from $P_{L S W} \approx 27.1 \mathrm{MPa}$ to $P_{L S W} \approx 48.6 \mathrm{MPa}$.

The damping factor of the LSW peak pressure due to presence of single cavitation bubbles based on definition in 5.6 .3 is $R_{L S W} \approx 58 \%$.

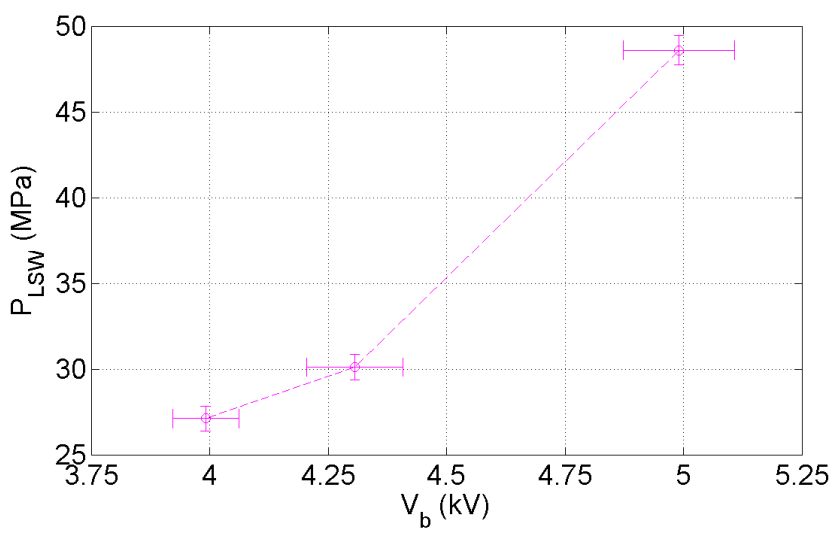

Figure 5.32: Lithotripter shock wave (LSW) peak pressure in the presence of the bubble. The dashed line connects the data points to guide the eye. Normalized LSW arrival time is $\alpha=0.12$.

\subsection{Conclusions}

The experimental results illustrated in sections 5.6 and 5.8 provide the possibility of investigating the effect of bubble oscillation phase at the moment of the lithotripter shock wave (LSW) impact. Thus, it can be concluded that for each specific LSW strength and the same bubble sizes at the moment of the shock impingement $(\approx 0.8 \mathrm{~mm})$, initially collapsing bubbles (see section 5.6 ) result in a more violent forced bubble collapse compared to the initially expanding cavities (see section 5.8). Therefore, stronger peak shock pressures and also more reductions of collapse time (the time interval between LSW impact and forced bubble collapse, $\Delta t_{i f}$ ) is expected in the case of the bubble being in the collapse phase at the moment of LSW arrival. The value of parameter $\Delta t_{i f}$ for initially expanding cavities is about $1.5-2.7$ times that of initially collapsing bubbles (see table 5.5).

The reason for such differences is the fact that for the case of initially expanding cavities, the compression part of the LSW profile first tries to stop the ongoing bubble expansion and then forces the cavity to collapse. Thus, work is done against the expansion. This process takes a longer time in comparison to the initially collapsing bubbles being exposed to the shock waves. The tension 
tail of the LSW profile tends to retard the bubble collapse and this effect is more pronounced for initially expanding bubbles which have a higher chance to be affected by this part of LSW profile in comparison to initially collapsing cavities. Therefore, for initially expanding bubbles some part of the energy of the LSW is consumed against the expansion while for initially collapsing cavities, the whole positive part of LSW energy is used for forcing the bubble to collapse.

The amplitude of the shock wave pressure released from the forced bubble collapse can be enhanced by an amount of about 4 times that of free inertial implosion, in the case that the LSW impinges on the bubble at the moment of $102 \mu \mathrm{s}$ after laser breakdown. The parameter $\Delta t_{i f}$ is reduced up to about $1.1 \mu \mathrm{s}$. Therefore, it can be said that the most enhancement of the bubble collapse occurs in the case that the value of parameter $\Delta t_{i f}$ approaches the pulse duration of the positive part of the LSW profile.

For each specific shock arrival time, the pressure enhancement and reduction of collapse time are more significant by increasing the LSW strength. The peak pressure of the shock wave is damped out by an amount of up to about $70 \%$ (based on the definition given in 5.6.3) in presence of the laser-generated single bubbles. The positive part of the LSW profile becomes broadened after passage through the single bubbles, which is due to both diffraction and absorption effects.

After the moment that the bubble reaches to its minimum size which forms a disk-like pattern, a shock-induced liquid jet with the velocity of up to about $580 \mathrm{~m} / \mathrm{s}$ (averaged over $0.6 \mu \mathrm{s}$ ) is visible. For the case of $\alpha=0.91$, after the moment of $0.6 \mu \mathrm{s}$ from the LSW impact, there is nearly no outstanding bubble centroid migration, while for $\alpha=0.97$ the cavity centroid translates in the direction of the LSW propagation even before this moment ( $\alpha$ is the normalized LSW arrival time). The slope of migration of the bubble centroid increases with the shock wave energy. The bubble centroid is translated up to about $0.5 \mathrm{~mm}$ after $6.6 \mu \mathrm{s}$ from the moment of the LSW impact. The cavity centroid migration velocity for the case of $\alpha=0.97$ is larger than that for $\alpha=0.91$. This also shows that the bubble is more affected by the LSW, if the shock arrives at the moment when the bubble is in its very late collapse phase. 


\section{Chapter 6}

\section{Shock wave - bubble interaction in the vicinity of a rigid boundary}

The existence of a solid interface near to a spherical cavitation bubble breaks the spherical symmetry of the problem. It leads to distortion of the bubble at collapse. In this case, the bubble contents become less compressed. The peak pressure of the wave emitted from cavitation bubble collapse is less compared to the inertial cavity implosion, due to bubble deformation [74, 7]. Measurements of Shima et al. [80] in 1981 and Vogel and Lauterborn in 1988 [97] show that shock waves can be emitted even from collapse in contact with a boundary [74]. Fluid flow between wall and the bubble is being retarded as a consequence of breaking the spherical symmetry. The oscillation frequency of the bubble is reduced and then, the collapse time of the cavity is prolonged [74, 83]. As a liquid jet impacts on the opposite bubble wall, a shock wave is emitted which propagates toroidally [59]. Ohl et al. [70] called that a "jet torus shock wave". The water-hammer pressure provides a way to describe the development of this shock wave. Soon after origination of the jet torus shock, a second "tip bubble shock wave" develops from the lowest point of the cavity [59].

\subsection{Method}

As a first test case, the dynamics of laser-generated single cavitation bubbles near to a solid interface is considered. Shadowgraph images are taken with a CCD camera (pco. imaging sensicam, Germany) using its single frame mode. For back illumination, an LED flash lamp with pulse duration of $\approx 10 \mathrm{~ns}$ (full width at half maximum (FWHM)) is used. In order to insure reproducibility of the laser-induced bubbles, the pressure field is recorded simultaneously by a hydrophone (Reson, TC4038) to control the bubble collapse time. For creation of image sequences, bubbles with minimum deviation in their collapse times are selected.

Regarding the effect of lithotripter shock waves (sections 6.3 and 6.4), the dynamics of laser-generated single cavitation bubbles in the vicinity of a solid boundary under the influence of lithotripter shock waves is investigated. The same experimental setup as described in 5.1 is used inserting a wall in vicinity of the laser focus. The normal vector to the wall is perpendicular to the direction of LSW propagation. A schematic view regarding orientation of shock wave passage and interface position, is displayed in Figure 6.1. 


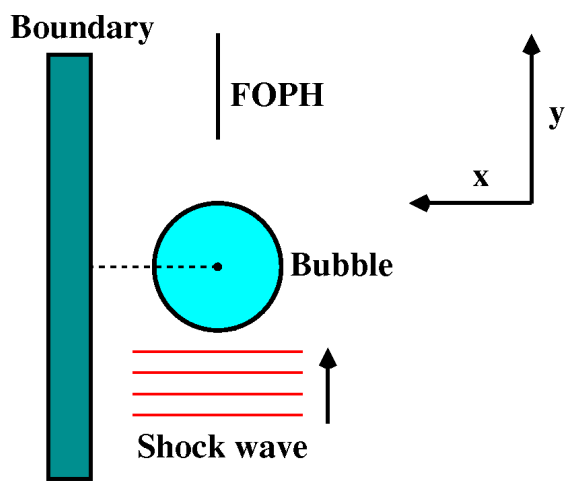

Figure 6.1: Schematic image related to location of rigid wall and direction of lithotripter shock wave (LSW) passage.

\subsection{Experimental results: part I}

Figure 6.2 illustrates dynamics of a laser-induced cavitation bubble near to a solid wall. Dimensionless distance between the cavity and the wall is defined as $\gamma=d / R_{\max } \approx 2$, where $d$ is the distance of the bubble to the boundary, and $R_{\max }$ being the maximum bubble radius in the free field. It is worth mentioning that in this case, no external shock wave has been applied. Development of jet torus shock wave is observed in this Figure.

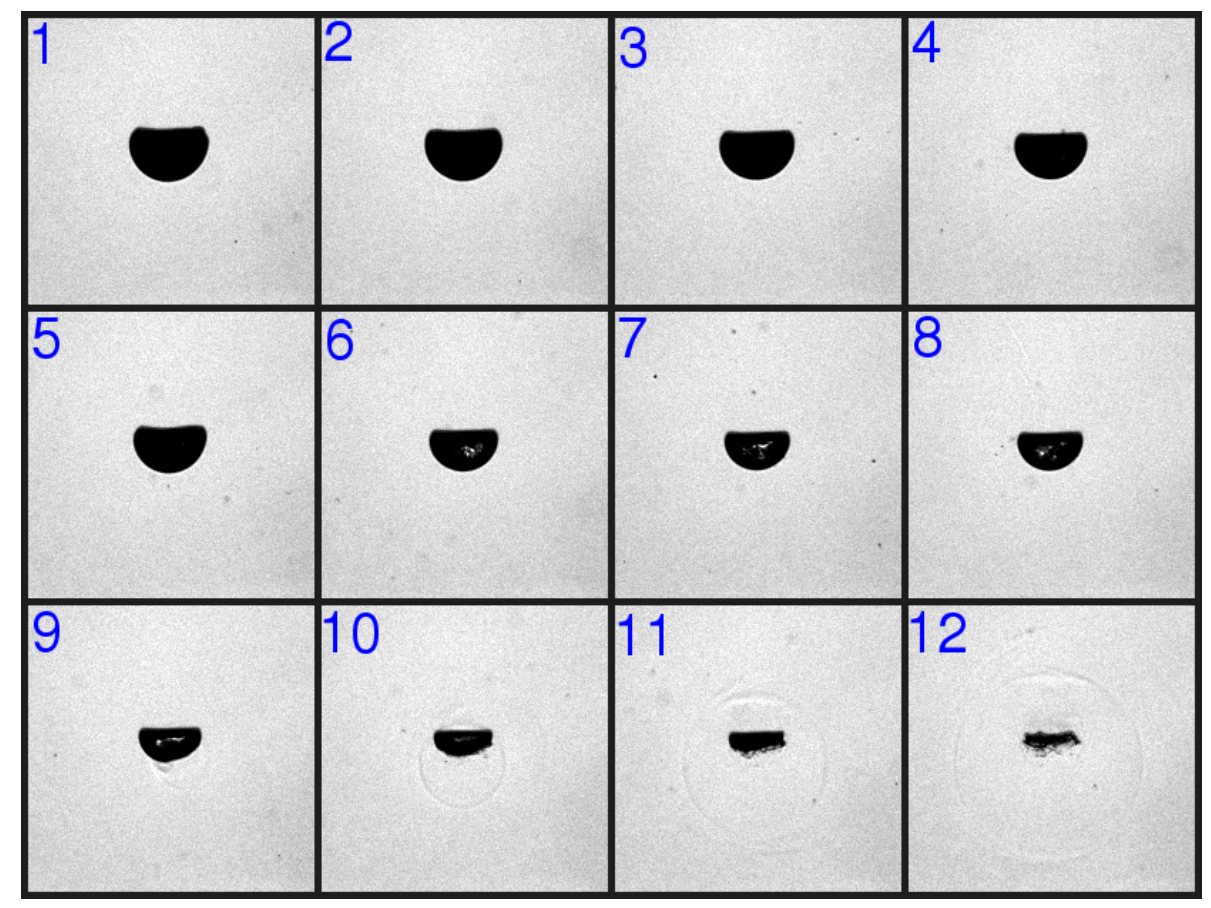

Figure 6.2: Dynamics of a laser-generated single cavitation bubble in the vicinity of a solid wall. Dimensionless distance is $\gamma \approx 2$ and the interframe time being $\Delta t=100$ ns. Frame width and hight are $\approx 1.9 \mathrm{~mm}$. 


\subsection{Experimental results: part II}

In this part, cavitation bubble dynamics near a rigid boundary in exposure to a lithotripter shock wave (LSW) is studied. Maximum bubble radius in free field $R_{\max }$ is given by the Rayleigh's formula [75]:

$$
R_{\max }=\frac{1}{0.915} \sqrt{\left(\frac{p-p_{v}}{\rho}\right)} t_{c}
$$

where $p$ is the static pressure at infinity, $p_{v}$ and $\rho$ are the vapor pressure and density of water, respectively. The parameter $t_{c}$ corresponds to half of the period of the first bubble oscillation cycle, which is half of the time between laser breakdown and first bubble collapse. This time interval is derived by the acoustic field measurements with fiber optic probe hydrophone (FOPH300) at distance of $d \approx 1.3 \mathrm{~mm}$ above the bubble centroid. Free bubble oscillation without the wall has a period of $2 t_{c}=105.2 \pm 3.8 \mu$ s and the pulse amplitude of free collapse shock wave in absence of the boundary is $P_{c}^{\prime}=7.4 \pm 1.2 \mathrm{MPa}$ (based on 20 measurements). By considering the appropriate values in equation 6.1, the maximum bubble radius is derived as $R_{\max } \approx 570 \mu \mathrm{m}$. Note that equation 6.1 holds just for free bubble oscillation.

The presence of a wall retards the bubble implosion and results in a longer collapse time compared to the free field case. Nevertheless, the LSW tends to accelerate the oscillation. Therefore, a counteraction between the influence of the wall and the lithotripter shock wave (LSW) is expected. The bubble translates to the wall (to the left side in Figure 6.1), due to the existence of the boundary. It also migrates in the direction of LSW propagation (to the top in this Figure) because of LSW impingement. It can be concluded that, by selection of an optimal interpulse delay (delay between optical breakdown and LSW source trigger), an inclined bubble displacement may be observed as a superposition of both wall and LSW effects.

In this investigation, only one intensity level of the shock wave source is used. Peak voltage of back and front layers of the shock wave generator piezo elements, are $V_{b}=4.46 \pm 0.13 \mathrm{kV}$ and $V_{f}=4.13 \pm 0.07 \mathrm{kV}$, respectively (Intensity level $I=15$ ). Pulse amplitude of the shock wave in the free field (no bubble) at a distance of $d \approx 1.8 \mathrm{~mm}$ above the focus is $88.3 \pm 2.1 \mathrm{MPa}$. The LSW impinges on the bubble at the moment of $t_{i} \approx 100 \mu$ s after laser breakdown. The normalized shock wave arrival time is $\alpha=t_{i} / 2 t_{c}=0.95$. Therefore, the single bubbles are already in their late collapse phase at the moment of shock wave impact. This is the time where the observation begins. At this moment the bubble vertical diameter is $714 \pm 18 \mu \mathrm{m}$ and its horizontal diameter being $726 \pm 17 \mu \mathrm{m}$.

Figure 6.3 gives high-speed shadowgraph images of shock wave - bubble interaction in the presence of a solid wall. They are taken with a very fast camera (Imacon 468, DRS Hadland Ltd.) described in section 5.1. In this image, the lithotripter shock wave impinges on single cavitation bubbles at the first frame 
for each image sequence $(a-h)$. The stand-off distance $\left(\gamma=d / R_{\max }\right)$ is varied in the range of $(1.17<\gamma<2.69)$ in 8 steps. The shock wave propagates from bottom to the top and the wall being to the left of each frame (not visible in these pictures). The interframe time is $\Delta t=1 \mu \mathrm{s}$ and the exposure time is set to $20 \mathrm{~ns}$. It can be seen that, by increasing the bubble distance from the wall, the collapse occurs earlier. This is due to the fact that the retardation effect of the boundary becomes less prominent at larger bubble distances. An inclination of induced liquid jets relative to the direction of LSW passage is visible for the lower $\gamma$ values and becomes more pronounced by decreasing the distance between cavity and the boundary. The bubble collapses to a disk-like shape which is tilted relative to the direction of shock wave passage. As the distance of the wall increases, the results become similar to the ones investigated in the previous chapter, which means that the effect of the lithotripter shock wave dominates the bubble dynamics.

The enhancement of the acoustic field is considered in section 6.3.1. A brief discussion about the relation between parameter $\gamma$ and the moment of bubble collapse is presented in section 6.3.2, as well as introducing the reflected shock wave from the wall. 


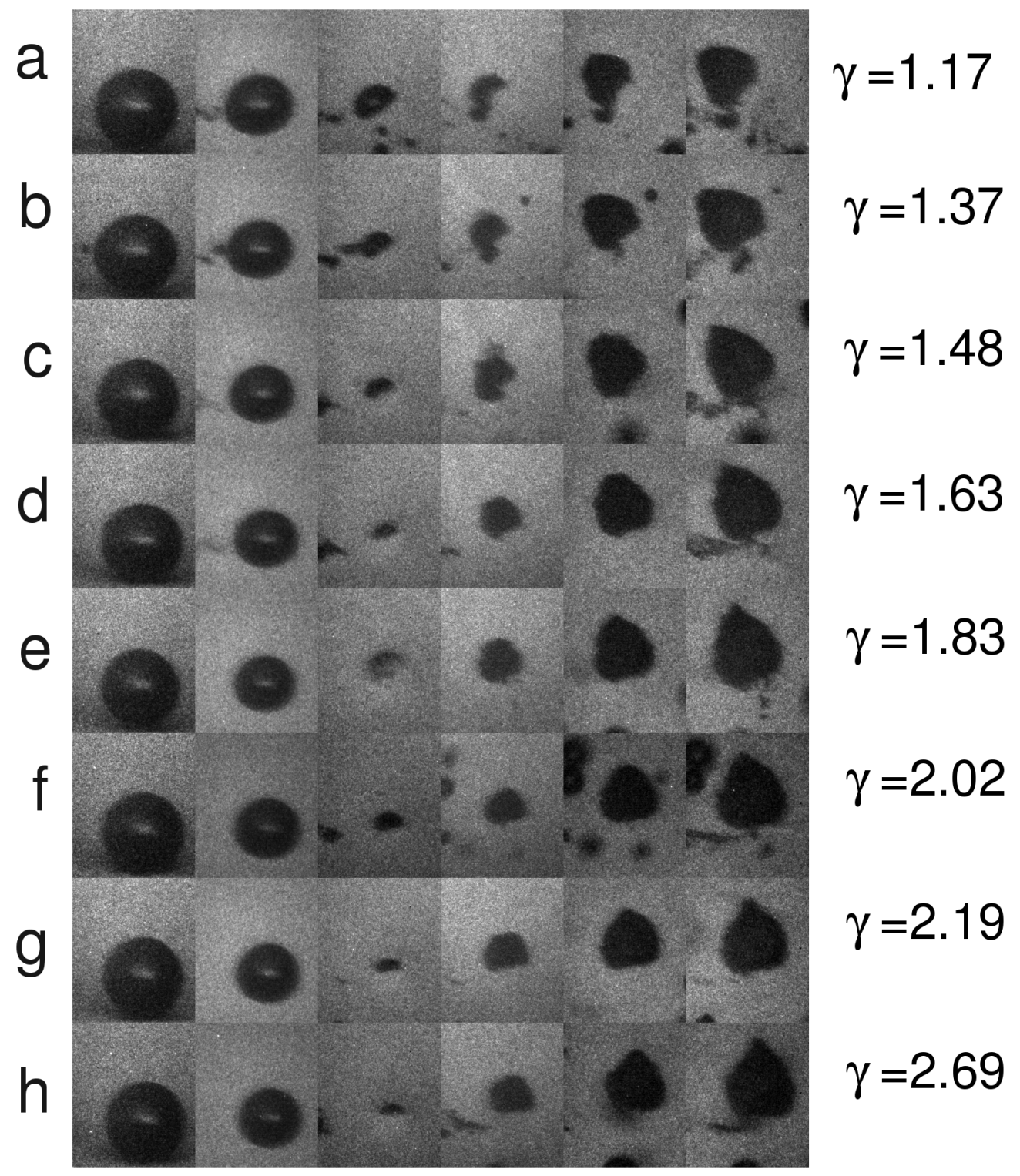

Figure 6.3: Dynamics of laser-generated single cavitation bubbles in the presence of a rigid boundary and lithotripter shock wave. Normalized LSW arrival time is $\alpha=0.95$. The LSW propagates from the bottom to the top and impinges on the bubbles at the first frame of each image sequence $(a-h)$. The solid wall (not visible here) is located at the left side. Interframe time is $1 \mu \mathrm{s}$ and the exposure time being $20 \mathrm{~ns}$. The frame width and height are $1.10 \mathrm{~mm}$ and $1.29 \mathrm{~mm}$, respectively. Initial bubble diameter in vertical direction is $714 \pm 18 \mu \mathrm{m}$ and its initial horizontal diameter being $726 \pm 17 \mu \mathrm{m}$. The peak pressure of the shock wave in the free field (no bubble) at a distance of $d \approx 1.8 \mathrm{~mm}$ above the focus is $88.3 \pm 2.1 \mathrm{MPa}$. 
The acoustic wave profile recorded at $d \approx 1.3 \mathrm{~mm}$ above the single cavitation bubble centroids is illustrated in Figure 6.4. The results are shown for four values of dimensionless bubble distances $(\gamma)$. The shock wave energy is constant. The pulse amplitude of the shock wave in the free field (no bubble) at a distance of $d \approx 1.8 \mathrm{~mm}$ above the focus is $88.3 \pm 2.1 \mathrm{MPa}$. By increasing the bubble distance to the wall, the collapse takes place earlier.
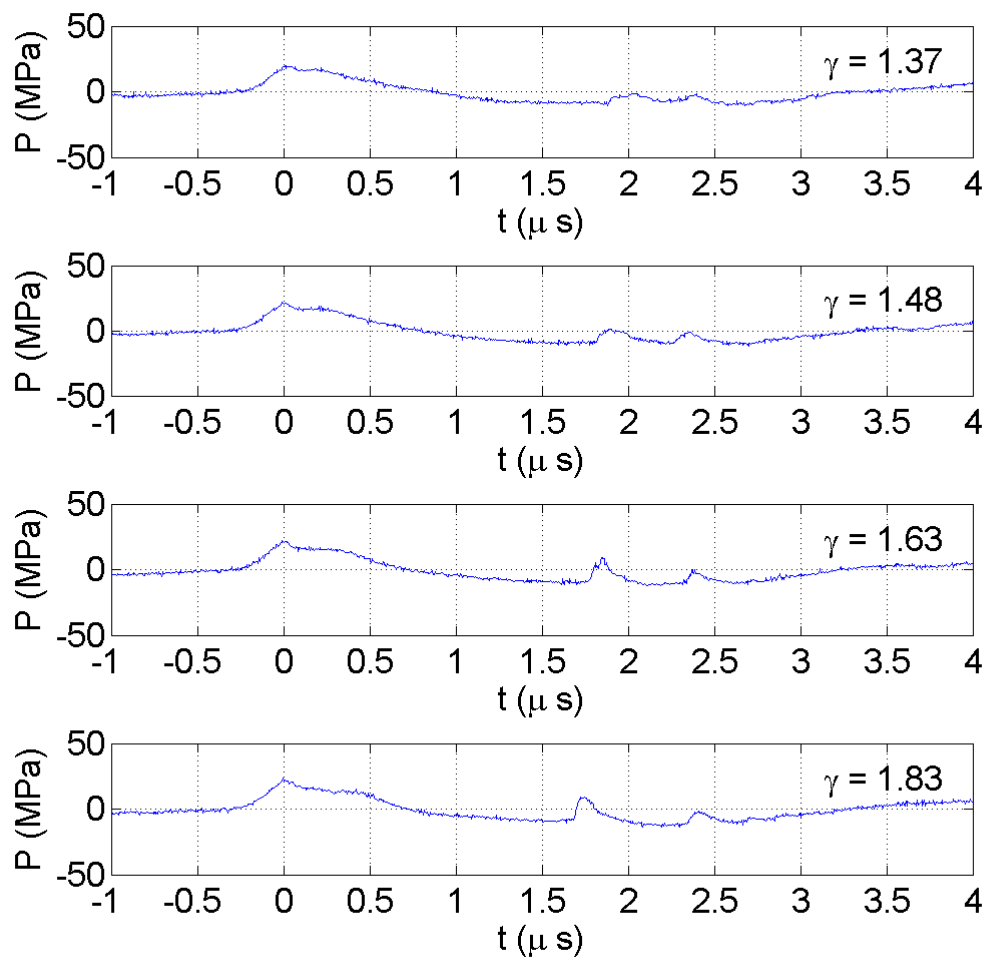

Figure 6.4: Time history of the pressure wave at $d \approx 1.3 \mathrm{~mm}$ from the bubble centroid. The moment of $t=0$ coincides with the peak pressure wave related to LSW. The second peak represents the forced bubble collapse. The third maximum corresponds to the reflection of the collapse shock from the wall. The second and third peaks shift to the left by increasing $\gamma$. 


\subsubsection{Pressure enhancement}

The amplitude of the shock wave emitted from bubble collapse $P_{c}$, divided by the peak pressure from inertial cavity implosion $\left(P_{c}^{\prime}=7.4 \pm 1.2 \mathrm{MPa}\right)$, is illustrated in Figure 6.5. The shock wave released from bubble collapse for $\gamma=$ 1.17 is so weak that it can not be resolved in the current analysis. For the case of $\gamma=1.37$ there is nearly no net pressure enhancement $\left(R=P_{c} / P_{c}^{\prime}=1.04\right)$. It might be concluded that the existence of the wall cancels out the effect of the LSW in enhancement of the shock wave emitted from cavity collapse.

The shock wave pulse amplitude is enhanced by a factor of $\approx 3.5$ for the case of $\gamma=2.69$. These results confirm that by increasing the bubble distance to the wall and therefore parameter $\gamma$, the effect of the LSW impact on the bubble becomes more significant with the result of a larger collapse pulse amplitude and then, a more violent bubble implosion occurs.

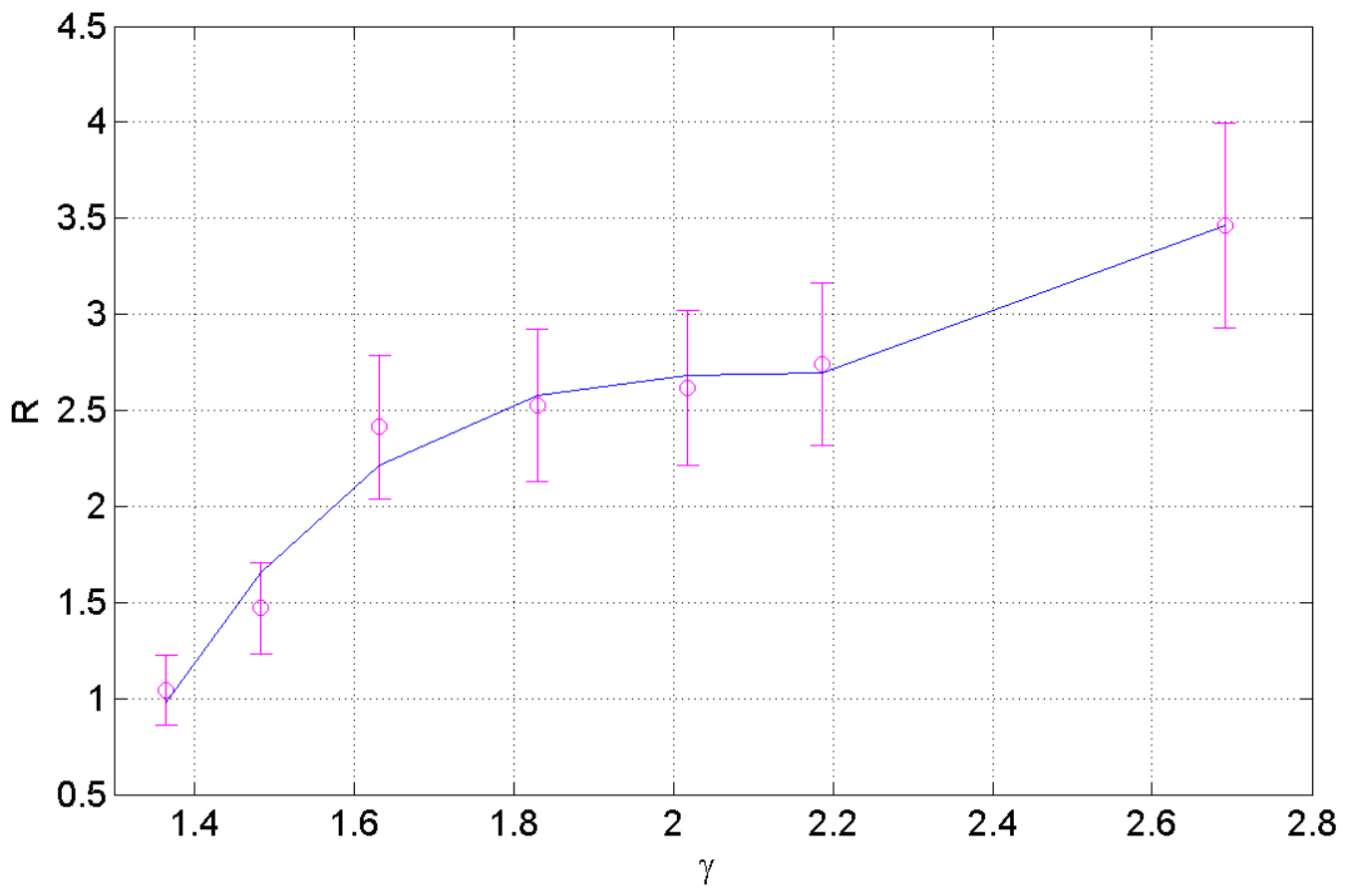

Figure 6.5: Enhancement of the shock wave pulse amplitude relative to single cavity implosion under the effect of LSW impingement near to a solid wall. Error bars are related to standard deviation of the experimental data. The solid line represents a cubic fit to measurement results. Normalized LSW arrival time is $\alpha=0.95$. 


\subsubsection{Reduction of collapse time}

The delay time $\Delta t_{i f}$ between the moment of LSW impingement on the bubble and the time that the shock wave is released from the forced cavity collapse (first and second peaks in Figure 6.4) is plotted for several stand-off distances $(\gamma)$, in Figure 6.6. The parameter $\Delta t_{i f}$ is reduced by increasing $\gamma$. This is related to the effect of LSW impact on the bubble, being more pronounced by higher $\gamma$ values.

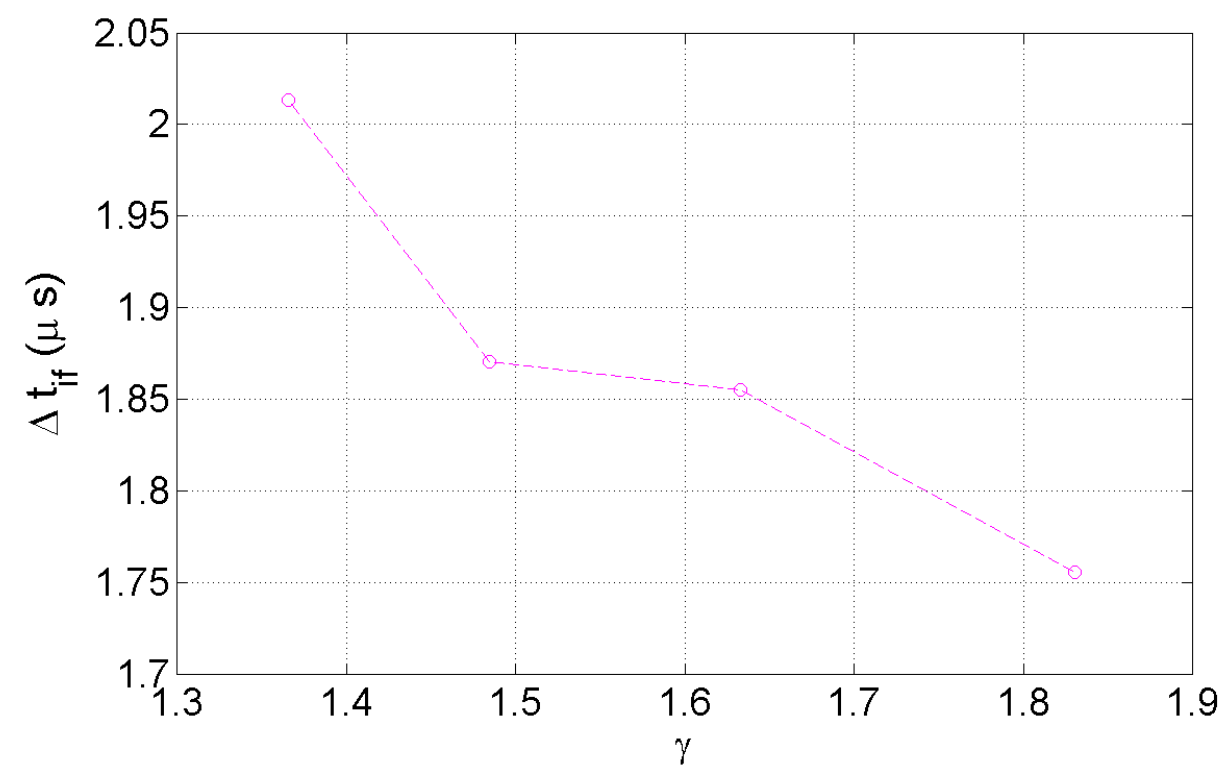

Figure 6.6: Reduction of bubble collapse time due to interaction between the LSW and laser-generated single cavitation bubbles, in presence of a rigid boundary.

The time interval between moments of laser breakdown and bubble collapse is $100+\Delta t_{\text {if }}$ (in $\mu \mathrm{s}$ ) which is actually less than that for the case of free collapse (i.e. $\approx 105.2 \mu \mathrm{s}$ ).

As the shock wave emitted from bubble collapse expands nearly spherically, one wave arrives directly at the hydrophone tip (at moment of $t=t_{1}$ ). The wave reflected by the boundary hits the hydrophone at a later time $t=t_{2}$. The delay time between arrival of these two shock waves at the hydrophone tip $\Delta t_{c}=t_{2}-t_{1}$ is depicted in Figure 6.7. This delay time $\left(\Delta t_{c}\right)$ is increasing by larger values of $\gamma$. There is a linear relation between parameters $\Delta t_{c}$ and stand-off distance $\gamma$, which is quite to be expected. Equation of the fitted line to the experimental data is: $\Delta t_{c}(\mu \mathrm{s})=(0.670 \pm 0.0327) \gamma-0.5510 \pm 0.0581$. 


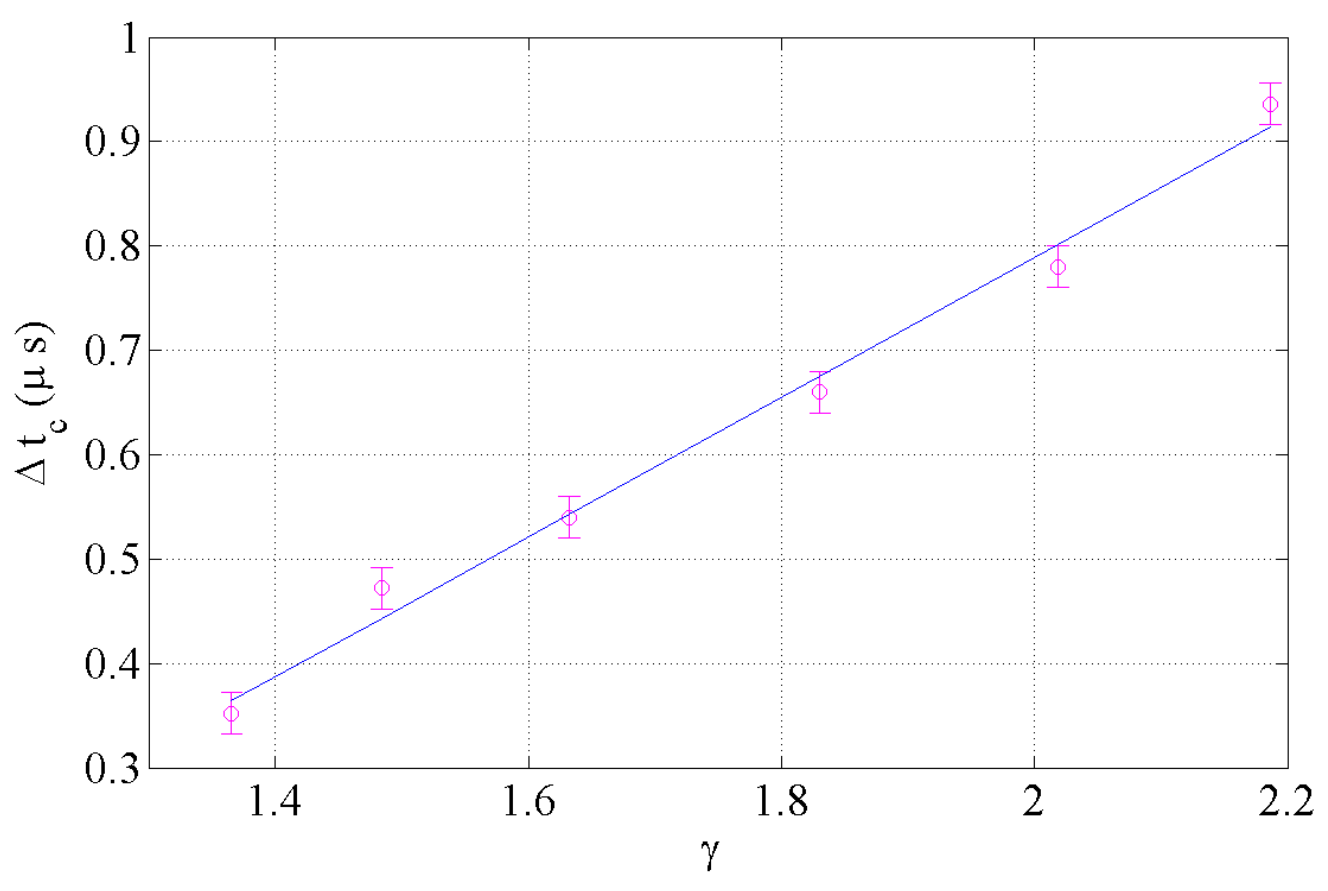

Figure 6.7: Delay time between moments of collapse shock wave emission and its reflection from the wall $\Delta t_{c}$, as a function of $\gamma$. The solid line is a linear fit to the measured data. Error bars show standard deviation of the experimental results.

\subsubsection{Bubble migration}

In order to study bubble dynamics in shock wave - bubble interaction in the vicinity of a solid interface with the geometry shown in Figure 6.1, the spatial position of the bubble tip as well as the migration of the cavity centroid are investigated.

Figure 6.8 illustrates the location of the bubble tip in $x$ direction (to the wall), relative to its initial position. The initial values are related to the moment of LSW impingement. This moment corresponds to the first frame of each image sequence in Figure 6.3.

The average velocity of the bubble tip over $\Delta t=5 \mu$ s after the LSW impingement for $\gamma=1.37$ is $V_{x} \approx 91.1 \mathrm{~m} / \mathrm{s}$. The cavity tip is translated to the wall up to $\Delta x \approx 0.46 \mathrm{~mm}$ at the moment of $t=5 \mu$ s after the shock wave arrival. Nevertheless, in the case of $\gamma=2.69$, there is nearly no significant displacement of the cavity tip to the wall. This fact indicates that by larger values of $\gamma$, the bubble dynamics in governed by the effect of LSW rather than the boundary. 


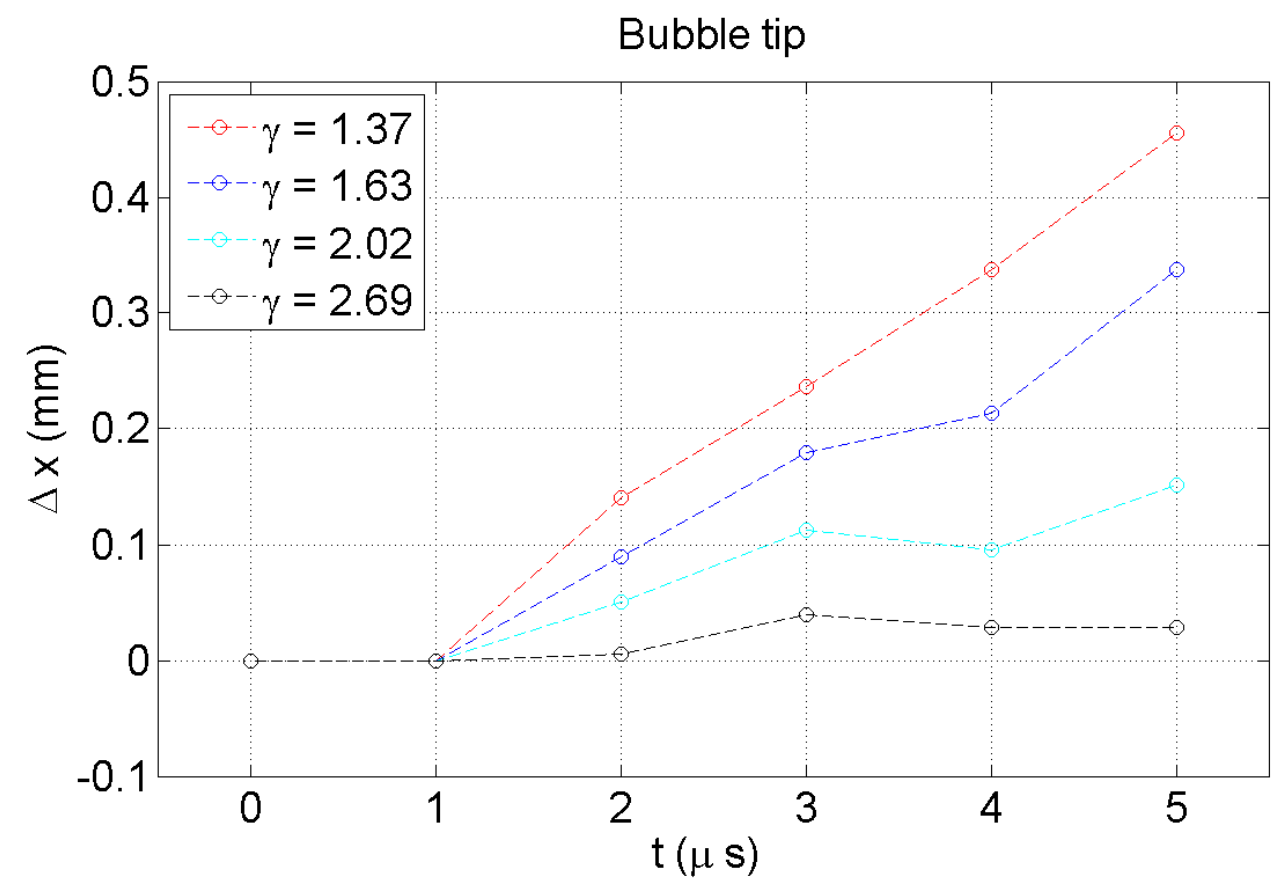

Figure 6.8: Migration of the bubble tip to the wall. The moment of $t=0$ corresponds to the time of shock wave arrival. The dashed lines connect the data points to guide the eye.

Displacements of the bubble centroid in horizontal (to the wall) and vertical directions are plotted in Figures 6.9 and 6.10. Similar to the case of the tip movement, there is almost no significant migration of the bubble centroid to the wall (in $x$ direction) in the case of $\gamma=2.69$. The cavity centroid is displaced by up to $\Delta x \approx 0.25 \mathrm{~mm}$ at $t=5 \mu$ s from the moment of shock wave impact. The bubble translates to the top ( $y$ direction) more rapidly. It is moved up to $\Delta y \approx 0.41 \mathrm{~mm}$ after $5 \mu$ s from the shock wave exposure, which corresponds to a velocity of $V_{y} \approx 82 \mathrm{~m} / \mathrm{s}$.

It can be seen that by lower bubble distances to the wall, the amount of migration of the cavity centroid to the boundary becomes comparable to its displacement in the direction of shock wave passage (at $t=5 \mu \mathrm{s}$ ). By increasing the wall distance, the difference of the translation of the bubble centroid in two directions rises. 


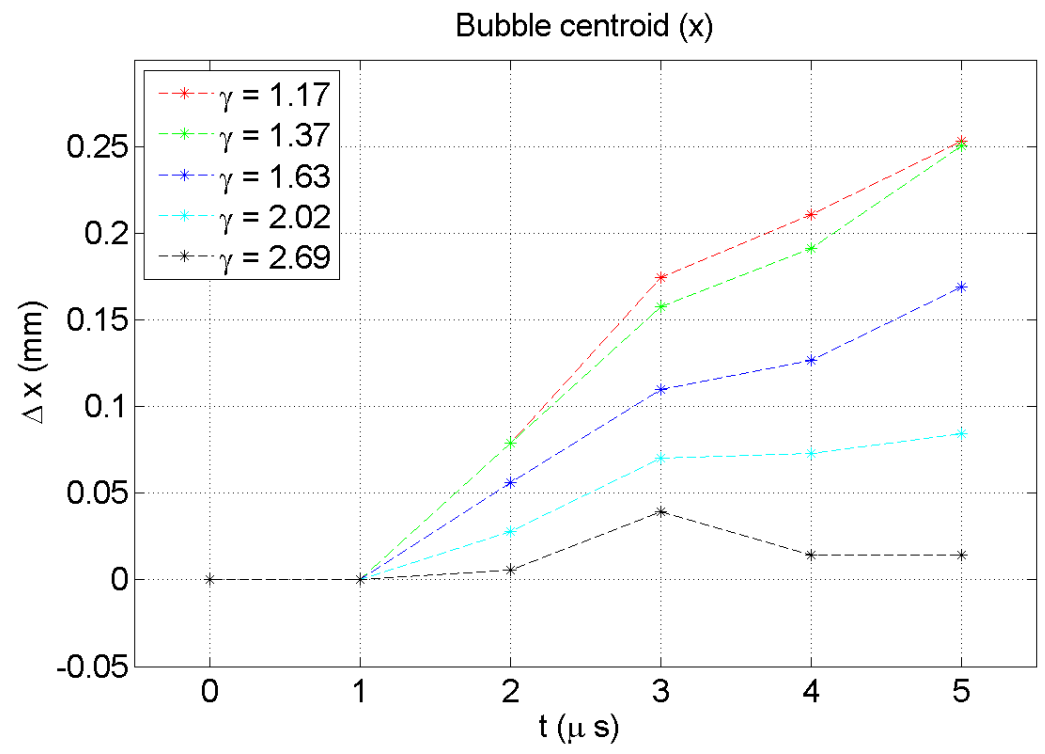

Figure 6.9: Migration of the bubble centroid to the wall. The moment of $t=0$ is related to the time of shock wave impact.

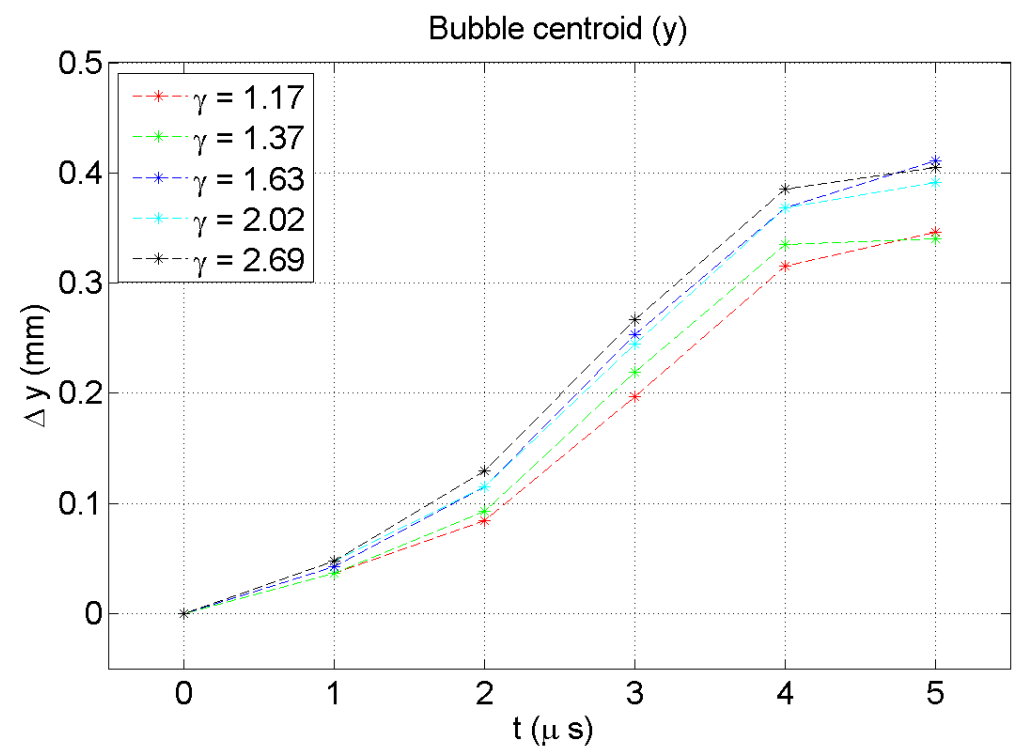

Figure 6.10: Migration of the bubble centroid in direction of the lithotripter shock wave passage. The moment of $t=0$ is related to the time of shock wave arrival. 
The non-zero cavitation bubble tip velocity in $x$ direction is a consequence of the inclination of the cavity tip with respect to the direction of LSW passage $(y)$. This phenomenon is observed in Figure 6.3 which shows that tendency of the bubble tip to the boundary is counteracted by increasing the stand-off distance $\gamma$. The inclination angle $\theta$ is defined as the angle between the direction of shock wave propagation and orientation of the bubble tip toward the wall. It is depicted in Figure 6.11 for different dimensionless distances. In the case of $\gamma=1.17$, the bubble is tilted by an amount of $\theta \approx 46.79^{\circ}$. The inclination vanishes $\left(\theta \approx 2.27^{\circ}\right)$ for the case of $\gamma=2.69$ which shows that the effect of shock wave is prominent compared to the wall.

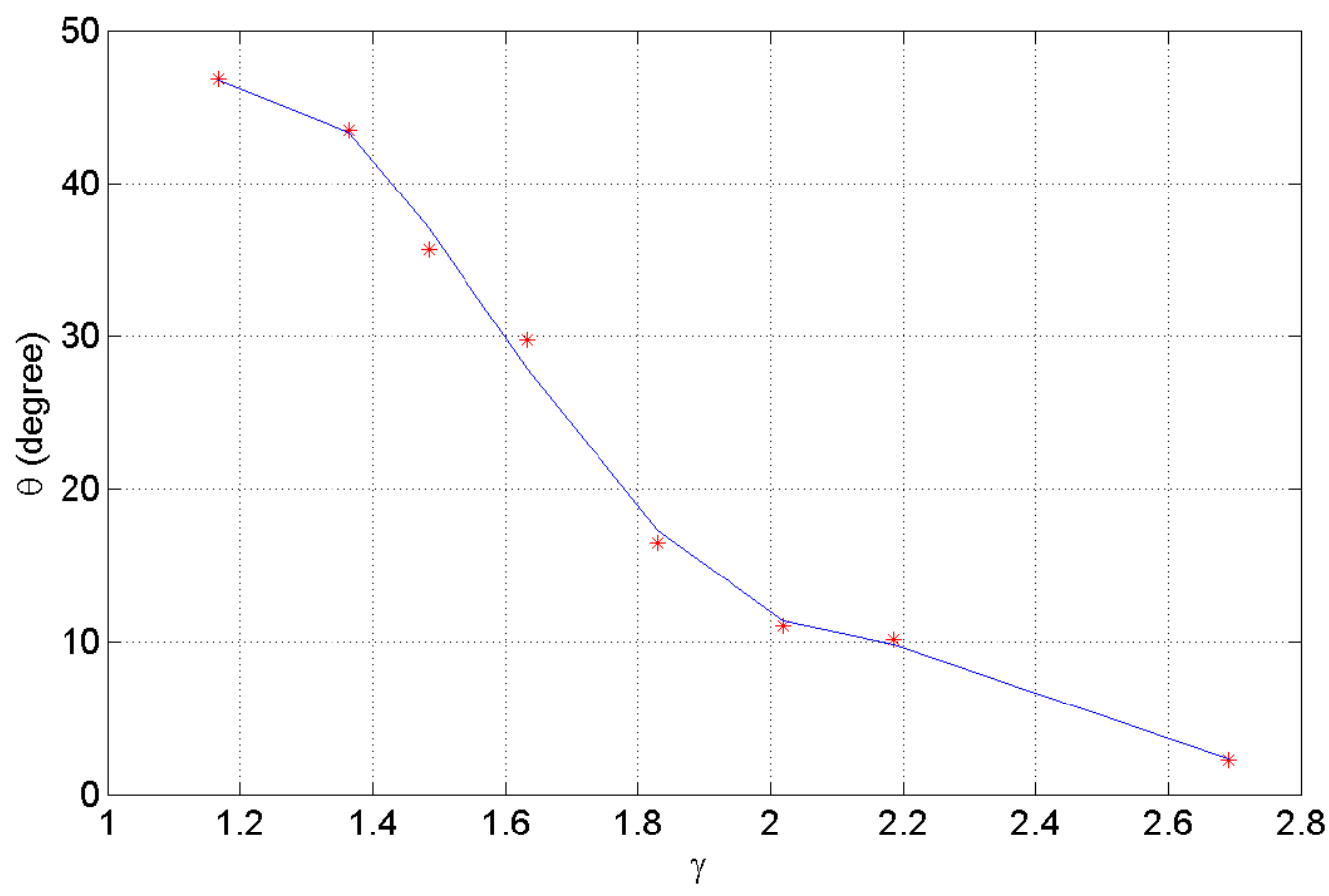

Figure 6.11: Inclination of the bubble tip relative to the direction of shock wave propagation. The data points are related to the last frame of each image sequence in Figure 6.3, which are related to the moment of $t=5 \mu \mathrm{s}$ after the lithotripter shock wave impact. The solid line is a polynomial fit of the 4th order to the experimental data. 


\subsection{Experimental results: part III}

As a third experimental test case, the effect of oscillation phase is considered. The distance between the location of the bubble generation and the interface is kept constant at $d \approx 0.82 \mathrm{~mm}$. Averaged over 20 measurements, the inertial bubble collapse time is $2 t_{c}=99.2 \pm 3.6 \mu \mathrm{s}$, and the pressure pulse amplitude of free bubble implosion is $P_{c}^{\prime}=5.7 \pm 0.9 \mathrm{MPa}$.

The mentioned collapse time corresponds to a bubble maximum radius of $R_{\text {max }} \approx 536 \mu \mathrm{m}$ in an undisturbed medium (using the Rayleigh formula [75]). By considering the maximum bubble radius and distance of bubble to the wall, the stand-off distance parameter is $\gamma \approx 1.52$.

The delay time between the moment of laser breakdown and the instant of LSW impingement on the bubble is defined as $t_{i}$ (see Figure 5.7). It is varied in 12 steps as $t_{i}=10,20 \ldots 100,105,110 \mu \mathrm{s}$. Bubble dynamics under the influence of lithotripter shock wave as well as a solid boundary is displayed in Figure 6.12, which shows shadowgraph images taken by a very high-speed camera (Imacon 468). The shock wave impinges on the single cavitation bubbles at the first frame of each image sequence $(a-l$ in Figure 6.12). Initially expanding as well as collapsing bubbles are investigated in this study. The vertical and horizontal cavity diameters at the moment of the shock wave impact $\left(D_{v 0}\right.$ and $\left.D_{h 0}\right)$ are in the ranges of $0.55 \mathrm{~mm}<D_{v 0}<1.14 \mathrm{~mm}$ and $0.58 \mathrm{~mm}<D_{h 0}<1.04 \mathrm{~mm}$, respectively.

The bubble sizes at the moment of shock wave impact for applying the LSW arrival times of $t_{i}=10$ and $t_{i}=105 \mu \mathrm{s}$ are almost similar $\left(D_{v 0}=0.67\right.$ and $0.61 \mathrm{~mm}$ and $D_{h 0}=0.64$ and $0.65 \mathrm{~mm}$ ). These shock impact times are related to the image series of $a$ and $k$ in Figure 6.12. It can be seen in this Figure that although the bubble sizes at the moment of LSW impingement are close to each other, induction of the liquid jet is more pronounced in the case of $t_{i}=105 \mu \mathrm{s}$ ( $k$ in Figure 6.12). If the initial bubble is in the collapse phase, the LSW impact results in a more violent cavity implosion. But for $t_{i}=10 \mu \mathrm{s}$ ( $a$ in Figure 6.12), the initial bubble is in its expansion phase. Work is done by the LSW in order to stop the expansion of the cavity before a forced bubble collapse takes place. Then, a weaker collapse is expected. These results are similar to those achieved in the previous chapter concerning shock wave - bubble interaction in absence of a wall.

It can be concluded that at this time scale (up to $t=5 \mu$ s after the shock impingement on the bubble), except for $t_{i}=10 \mu \mathrm{s}$, the liquid jet is only visible for the LSW arrival times in the range of $t_{i} \geq 90 \mu \mathrm{s}$, which correlate with the initially collapsing bubbles. The liquid jet is more prominent by decreasing the initial bubble sizes in the collapse phase. Inclination of the bubble tip with respect to the direction of LSW propagation is visible in these image sequences. Nevertheless, for the initial bubble sizes close to the maximum bubble radius, nearly no significant inclination is observed. 


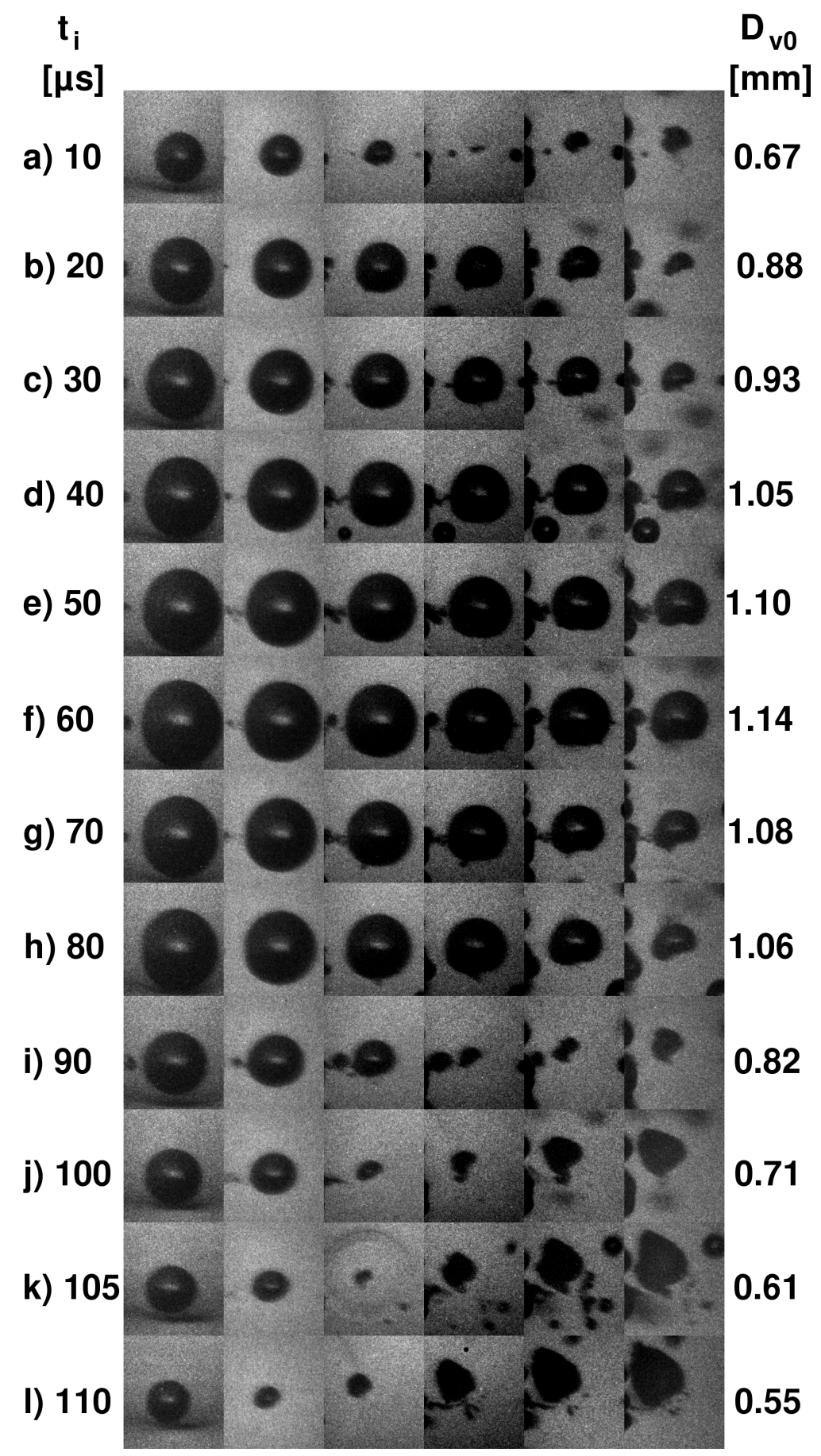

Figure 6.12: Shock wave - bubble interaction in presence of a solid boundary. The wall (not visible here), is located at the left side. Interframe time is $1 \mu \mathrm{s}$. Frame width is $1.29 \mathrm{~mm}$ and frame height being $1.46 \mathrm{~mm}$. The instant of shock wave impingement relative to the moment of laser breakdown is defined as $t_{i}$. At this time (at the first frames from $a$ to $l$ ), the bubble vertical diameter is given by $D_{v 0}$. 
The pressure wave profile (see Figure 6.13) is measured at the distance of $d \approx 1.33 \mathrm{~mm}$ above the laser-generated single cavitation bubble centroids in the vicinity of a solid boundary. It includes the LSW positive peak (first maximum) as well as the shock wave emitted from cavity collapse (second peak) and the succeeding reflected shock wave from the wall (third peak). Second and third maxima shift to the left by increasing the time of LSW impact $\left(t_{i}\right)$. This fact indicates more reduction in the time interval between the moment of LSW impact and the instant of forced bubble collapse $\left(\Delta t_{i f}\right)$ by larger values of $t_{i}$.
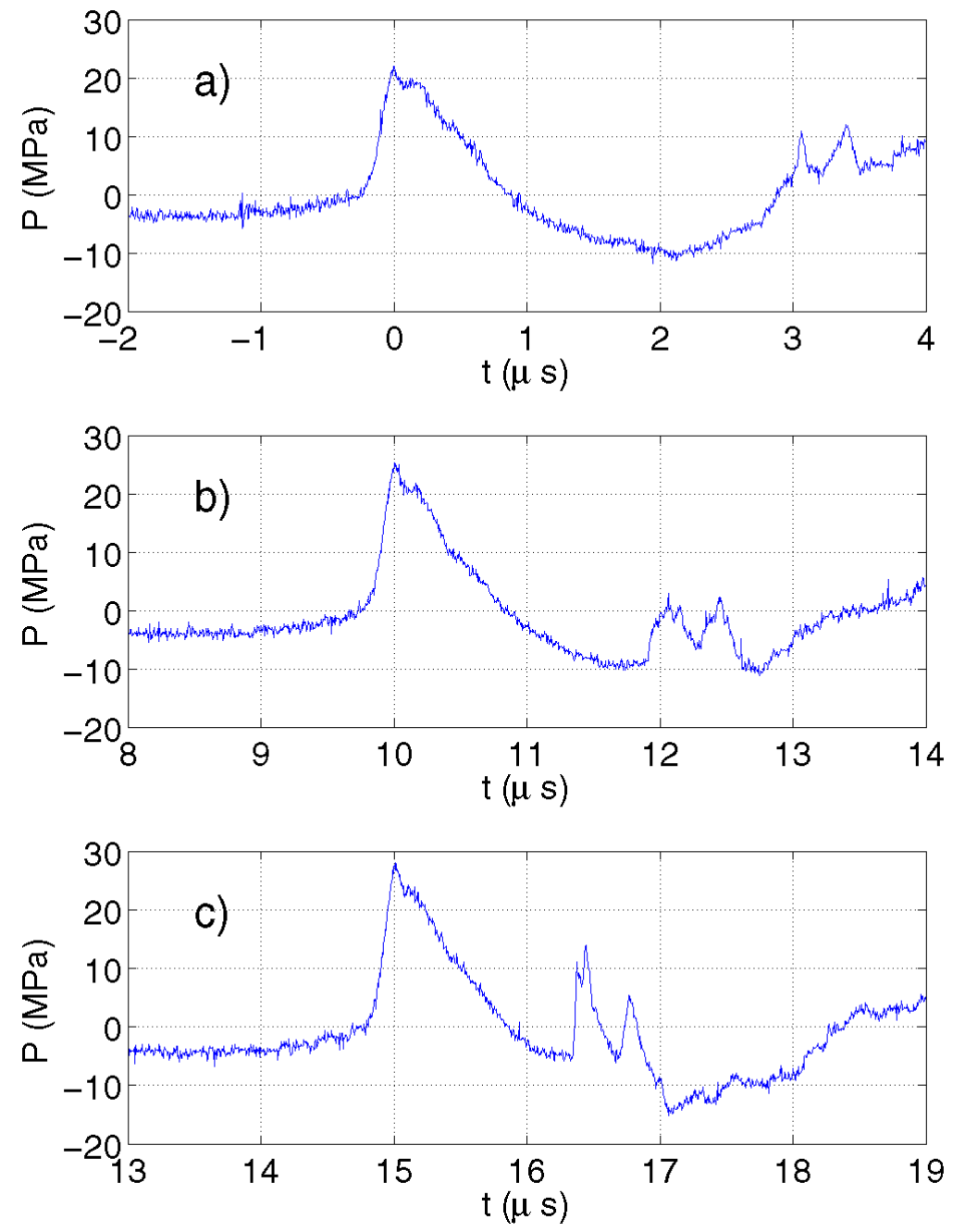

Figure 6.13: Pressure wave profile above the bubble. The LSW arrival times are $t_{i}=90(\mathrm{a}), 100(\mathrm{~b})$ and $105(\mathrm{c}) \mu \mathrm{s}$. The moment of $t=0$ relates to the LSW peak for $t_{i}=90 \mu \mathrm{s}$. 


\subsubsection{Pressure amplification and reduction of collapse time}

The parameter $t_{i}^{\prime}$ is defined as $t_{i}^{\prime}=\left(t_{i}-90\right) \mu \mathrm{s}$. The enhancement of the collapse shock wave amplitude due to the effect of LSW impact is depicted in Figure 6.14(a). It plots the ratio between the forced and inertial cavity collapse pulse amplitudes $\left(R=P_{c} / P_{c}^{\prime}\right)$. It has to be mentioned that for several values of $t_{i}$, the collapse shock is not distinguishable from the bias noise level of the FOPH. This may be due to the weakness of the shock wave emitted from the bubble implosion. Error bars are related to the bias noise level of the hydrophone signal. The amplitude of shock wave released from bubble collapse is amplified by a factor of up to $R \approx 3.44$.

The reduction in the delay between moments of LSW impingement and collapse shock $\left(\Delta t_{i f}\right)$ is illustrated in Figure 6.14(b), for three values of the parameter $t_{i}^{\prime}$. As shown in this Figure, there is a linear relation between parameters $\Delta t_{i f}$ and $t^{\prime}$. Equation of the fitted line to the experimental data points is $\Delta t_{i f}(\mu \mathrm{s})=(-0.1073 \pm 0.0053) t_{i}^{\prime}(\mu \mathrm{s})+3.079 \pm 0.0546$.

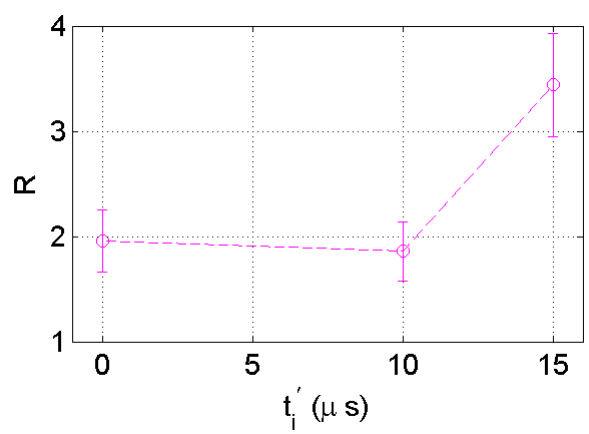

(a)

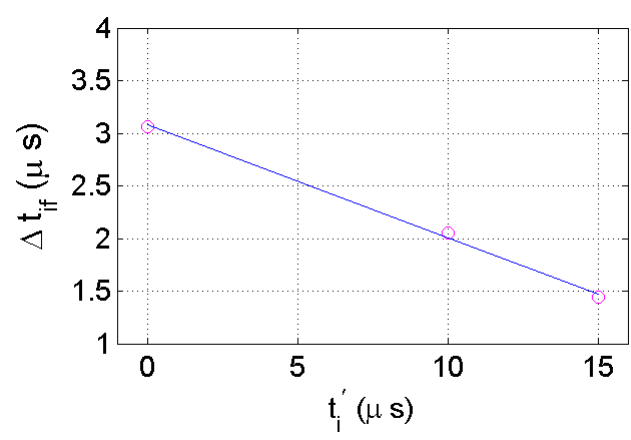

(b)

Figure 6.14: Enhancement of shock wave from cavity collapse 6.14(a) and reduction of bubble implosion time 6.14(b) in shock wave - bubble interaction in vicinity of a stiff wall. The dashed line in the upper graph connects the data points, while in the lower image the solid line represents a linear fit to the measurement results. 


\subsection{Conclusions}

By investigating the behavior of laser-generated single cavitation bubbles close to a solid boundary with the geometry illustrated in Figure 6.1, it seems to exist a counteraction between the effects of the solid wall and the impinging lithotripter shock wave. The LSW tends to translate the cavity in the direction of its propagation (i.e. $y$ direction in Figure 6.1), while the bubble can also migrate toward the neighboring rigid interface (i.e. $x$ direction in Figure 6.1). The bubble collapse time is reduced due to the interaction with the shock wave. Nevertheless, the presence of the wall leads to a longer collapse time.

For a constant shock energy, by increasing the stand-off distance $\gamma$ :

- the bubble implosion occurs earlier

- the forced collapse is enhanced

- the amount of the migrations of the bubble tip and the cavity centroid toward the wall are reduced.

These phenomena indicate that by increasing the stand-off distance, the bubble dynamics is governed by the effect of the LSW. For $\gamma=2.69$, there are almost no noticeable translations of the bubble tip and centroid toward the wall, which means that the liquid jet is developed nearly in the direction of the LSW passage (see Figure 6.3). However, the bubble tip is moved toward the interface by an amount of up to $\approx 0.5 \mathrm{~mm}$ after $5 \mu$ s from the LSW impact, for the case of $\gamma=1.37$.

The cavity is inclined up to $\theta \approx 45^{\circ}$ relative to the original direction of the incident shock, which shows the effect of both wall and LSW for $\gamma=1.17$. The inclination is decreasing with $\gamma$ and drops to about zero for $\gamma=2.69$.

Comparing two similar initial bubble sizes being in different oscillation phases (image sequences $a$ and $k$ in Figure 6.12), it can be said that the bubble collapse is more violent for an initially collapsing cavity. This result is in agreement with the ones presented in the previous chapter. 


\section{Bibliography}

[1] Introduction to the Physics and Technology of Extracorporeal Shock Wave Therapy (ESWT/CSWT). Storz Medical AG, Kreuzlingen, Switzerland, October 2003.

[2] I. Akhatov, O. Lindau, A. Topolnikov, R. Mettin, N. Vakhitova, and W. Lauterborn. Collapse and rebound of a laser-induced cavitation bubble. Phys. of Fluids, 13(10):2805-2819, 2001.

[3] P. Augat and L. Claes. Influence of water temperature on pressure pulses generated by an electromagnetic type lithotripter. Ultrasound in Med. and Biol., 21(1):89-96, 1995.

[4] G. J. Ball, B. P. Howell, T. G. Leighton, and M. J. Schofield. Shockinduced collapse of a cylindrical air cavity in water: a Free-Lagrange simulation. Shock Waves, 10:265-276, 2000.

[5] P. A. Barnes and K. E. Rieckhoff. Laser induced underwater sparks. Appl. Phys. Lett., 13:282-284, 1968.

[6] F. Belgiorno, S. Liberati, M. Visser, and D.W. Sciama. Sonoluminescence: two-photon correlations as a test of thermality. Phys. Lett. A, 271:308 $313,2000$.

[7] T. B. Benjamin and A. T. Ellis. The collapse of cavitation bubbles and the pressures thereby produced against solid boundaries. Phil. Trans. R. Soc. Lond. A, 260:221-240, 1966.

[8] J. P. Best and J. R. Blake. An estimate of the kelvin impulse of a transient cavity. J. Fluid Mech., 261:75-93, 1994.

[9] J. R. Blake, B. B. Taib, and G. Doherty. Transient cavities near boundaries. Part 1. Rigid boundary. J. Fluid Mech., 170:479-497, 1986.

[10] N. K. Bourne and J. E. Field. Shock-induced collapse of single cavities in liquids. J. Fluid Mech., 244:225-240, 1992.

[11] F. P. Bowden. The Formation of Microjets in Liquids under the Influence of Impact or Shock. Phil. Trans. R. Soc. London, A, 260:94-95, 1966.

[12] F. P. Bowden and J. H. Brunton. The Deformation of Solids by Liquid Impact at Supersonic Speeds. Proc. R. Soc. London, A, 263:433-450, 1961. 
[13] F. P. Bowden and M. P. McOnie. Cavities and Micro Munro Jets in Liquids: Their Role in Explosion. Nature, 206:380-383, 1965.

[14] C. E. Brennen. Cavitation and Bubble Dynamics. Oxford University Press, 1995.

[15] M. P. Brenner, S. Hilgenfeldt, and D. Lohse. Single-bubble sonoluminescence. Rev. Mod. Phys., 74:425-484, 2002.

[16] E. A. Brujan, K. Nahen, P. Schmidt, and A. Vogel. Dynamics of laserinduced cavitation bubbles near an elastic boundary. J. Fluid Mech., 433:251-281, 2001.

[17] E. A. Brujan, K. Nahen, P. Schmidt, and A. Vogel. Dynamics of laserinduced cavitation bubbles near elastic boundaries: influence of the elastic modulus. J. Fluid Mech., 433:283-314, 2001.

[18] J. H. Brunton. High speed liquid impact. Phil. Trans. R. Soc. Lond. A, 260:79-85, 1966.

[19] J. H. Brunton, M. P. W. Wilson, and J. J. Camus. The high-speed photography of liquid flow in collapsing cavities and impinging liquid drops. In Proceedings the 8th International Congress on High Speed Photography, Stockholm, Sweden (Wiley, London), pages 467-470, 1968.

[20] M. M. Chaudhri and J. E. Field. The role of rapidly compressed pockets in the initiation of condensed explosives. Proc. R. Soc. Lond. A., 340:113128, 1974.

[21] C. C. Church. A theoretical study of cavitation generated by an extracorporeal shock wave lithotripter. J. Acoust. Soc. Am., 86:215-227, 1989 .

[22] Robert H. Cole. Underwater Explosions. Princeton University Press, Princeton, 1948.

[23] A. J. Coleman, M. Whitlock, T. Leighton, and J. E. Saunders. The spatial distribution of cavitation induced acoustic emission, sonoluminescence and cell lysis in the field of a shock wave lithotripter. Phys. Med. Biol., 38:1545-1560, 1993.

[24] L. A. Crum. Tensile strength of water. Nature, 278:148-149, 1979.

[25] L. A. Crum. Acoustic cavitation series: part five. Rectified diffusion. Ultrasonics, 22:215-223, 1984.

[26] L. A. Crum and T. J. Matula. Sonoluminescence: Shocking Revelations. Science, 276:1348-1349, 1997. 
[27] J. P. Dear and J. E. Field. High-speed photography of surface geometry effects in liquid/solid impact. J. Appl. Phys., 63:1015-1021, 1988.

[28] J. P. Dear and J. E. Field. A study of the collapse of arrays of cavities. J. Fluid Mech., 190:409-425, 1988.

[29] Z. Ding and S. M. Gracewski. The behaviour of a gas cavity impacted by a weak or strong shock wave. J. Fluid Mech., 309:183-209, 1996.

[30] C. Eberlein. Theory of quantum radiation observed as sonoluminescence. Phys. Rev. A, 53(4):2772 -2787, 1996.

[31] A. T. Ellis, W. P. Gruber, and N. George. An experimental investigation of shock wave-bubble interaction and reflection holograms of long polymer molecules. Contract NONR 220(44) Report to Office of Naval Research (Pasadena), pages 1-32, 1968.

[32] Bengt O. Enflo and Claes M. Hedberg. Theory of Nonlinear Acoustics in Fluids. Kluwer Academic Publishers, Dordrecht, 2002.

[33] J. E. Field. The physics of liquid impact, shock wave interactions with cavities, and the implications to shock wave lithotripsy. Phys. Med. Biol., 36(11):1475-1484, 1991.

[34] D. J. Flannigan and K. S. Suslick. Plasma formation and temperature measurement during single-bubble cavitation. Nature, 434:52-55, 2005.

[35] J. G. Fujimoto, W. Z. Lin, E. P. Ippen, C. A. Puliafito, and R. F. Steinert. Time-resolved studies of Nd:YAG laser-induced breakdown. Plasma Formation, Acoustic Wave Generation, and Cavitation. Invest. Ophthalmol. Vis. Sci., 26:1771-1777, 1985.

[36] S. Gambihler, M. Delius, and J.W. Ellwart. Permeabilization of the plasma membrane of L1210 mouse leukemia cells using lithotripter shock waves. J. Membrane Biol., 141:267-275, 1994.

[37] N. Garcia and A. P. Levanyuk. Comment on "Sonoluminescence as Quantum Vacuum Radiation". Phys. Rev. Lett., 78(11):2268-2268, 1997.

[38] J. W. Grove and R. Menikoff. Anomalous reflection of a shock wave at a fluid interface. J. Fluid Mech., 219:313-336, 1990.

[39] Mark F. Hamilton and David T. Blackstock. Nonlinear Acoustics. Academic Press, San Diego, 1997.

[40] R. Hiller, K. Weninger, S. J. Putterman, and B. P. Barber. Effect of Noble Gas Doping in Single-Bubble Sonoluminescence. Science, 266:248-250, 1994. 
[41] J. A. Hossack and B. A. Auld. Improving the characteristics of a transducer using multiple piezoelectric layers. IEEE Trans. on Ultrasonics, Ferroelectrics and Frequency Control, 40(2):131-139, 1993.

[42] E. Johnsen and T. Colonius. Shock-induced collapse of a gas bubble in shockwave lithotripsy. J. Acoust. Soc. Am., 124(4):2011-2020, 2008.

[43] E. Klaseboer, S. W. Fong, C. K. Turangan, B. C. Khoo, A. J. Szeri, M. L. Calvisi, G. N. Sankin, and P. Zhong. Interaction of lithotripter shockwaves with single inertial cavitation bubbles. J. Fluid Mech., 593:33-56, 2007.

[44] E. Klaseboer, K. C. Hung, C. Wang, C. W. Wang, B. C. Khoo, P. Boyce, S. Debono, and H. Charlier. Experimental and numerical investigation of the dynamics of an underwater explosion bubble near a resilient/rigid structure. J. Fluid Mech., 537:387-413, 2005.

[45] T. Kodama and K. Takayama. Dynamic behavior of bubbles during extracorporeal shock-wave lithotripsy. Ultrasound in Med. and Biol., 24(5):723-738, 1998.

[46] T. Kodama, K. Takayama, and N. Nagayasu. The dynamics of two air bubbles loaded by an underwater shock wave. J. Appl. Phys., 80(10):5587-5592, 1996.

[47] T. Kodama, K. Takayama, and H. Uenohara. A new technology for revascularization of cerebral embolism using liquid jet impact. Phys. Med. Biol., 42:2355-2367, 1997.

[48] T. Kodama and Y. Tomita. Cavitation bubble behavior and bubbleshock wave interaction near a gelatin surface as a study of in vivo bubble dynamics. Appl. Phys. B, 70:139-149, 2000.

[49] M. Kornfeld and L. Suvorov. On the destructive action of cavitation. J. Appl. Phys., 15:495-506, 1944.

[50] K. H. Kuttruff. Pressure-induced interaction between bubbles in a cavitation field. J. Acoust. Soc. Am., 106:190-194, 1999.

[51] W. Lauterborn. High-speed photography of laser-induced breakdown in liquids. Appl. Phys. Lett., 21:27-29, 1972.

[52] W. Lauterborn. Kavitation durch Laserlicht. Acustica, 31:52-78, 1974.

[53] W. Lauterborn. Cavitation and Inhomogeneities in Underwater Acoustics. Springer Verlag, 1980.

[54] W. Lauterborn and H. Bolle. Experimental investigations of cavitationbubble collapse in the neighbourhood of a solid boundary. J. Fluid Mech., 72:391-399, 1975. 
[55] W. Lauterborn, T. Kurz, and I. Akhatov. Nonlinear Acoustics in Fluids. In: Springer Handbook of Acoustics, Ed. T. D. Rossing, chapter 8, pages 257-297. Springer, New York, 2007.

[56] W. Lauterborn, T. Kurz, R. Mettin, and C. D. Ohl. Experimental and Theoretical Bubble Dynamics. In: Advances in Chemical Physics Volume 110. Ed. by I. Prigogine and S. A. Rice, chapter 5, pages 295-380. John Wiley and Sons, Inc., 1999.

[57] W. Lauterborn, T.Kurz, C. Schencke, O. Lindau, and B. Wolfrum. LaserInduced Bubbles in Cavitation Research. In: Proc. IUTAM Symposium on Free Surface Flows, Birmingham, United Kingdom, 10-14 July 2000, ed. A.C. King, Y.D. Shikhmurzaev; Dordrecht : Kluwer Academic Publishers, Fluid Mechanics and its Applications. 62:169-176, 2001.

[58] T. Lepoint, D. De Pauw, F. Lepoint-Mullie, M. Goldman, and A. Goldman. Sonoluminescence: An alternative electrohydrodynamic hypothesis. J. Acoust. Soc. Am., 101:2012-2030, 1997.

[59] O. Lindau and W. Lauterborn. Cinematographic observation of the collapse and rebound of a laser-produced cavitation bubble near a wall. $J$. Fluid Mech., 479:327-348, 2003.

[60] D. Lohse. Sonoluminescence: Cavitation hots up. Nature, 434:33-34, 2005 .

[61] P. A. Lush. Impact of a liquid mass on a perfectly plastic solid. J. Fluid Mech., 135:373-387, 1983.

[62] C. L. Mader. Initiation of Detonation by the Interaction of Shocks with Density Discontinuities. Phys. Fluids, 8:1811-1816, 1965.

[63] T. J. Matula, R. A. Roy, and P. D. Mourad. Optical pulse width measurements of sonoluminescence in cavitation-bubble fields. J. Acoust. Soc. Am., 101(4):1994-2002, 1997.

[64] T. J. Matula, R. A. Roy, P. D. Mourad, W. B. McNamara, and K. S. Suslick. Comparison of Multibubble and Single-Bubble Sonoluminescence Spectra. Phys. Rev. Lett., 75:2602-2605, 1995.

[65] C. F. Naude and A. T. Ellis. On the mechanism of cavitation damage by non-hemispherical cavities collapsing in contact with a solid boundary. J. Basic Engng., Trans. ASME, D, 83:648-656, 1961.

[66] J. Noack and A. Vogel. Single-shot spatially resolved characterization of laser-induced shock waves in water. Appl. Opt., 37:4092-4099, 1998.

[67] B. E. Noltingk and E. A. Neppiras. Cavitation produced by Ultrasonics. Proc. Phys. Soc. (London). B63:674-685, 1950. 
[68] T. Obara, N. K. Bourne, and J. E. Field. Liquid-jet impact on liquid and solid surfaces. Wear, 186-187:388-394, 1995.

[69] C. D. Ohl and R. Ikink. Shock-Wave-Induced Jetting of Micron-Size Bubbles. Phys. Rev. Lett., 90(21):214502, 2003.

[70] C. D. Ohl, T. Kurz, R. Geisler, O. Lindau, and W. Lauterborn. Bubble dynamics, shock waves and sonoluminescence. Phil. Trans. R. Soc. Lond. A, 357:269-294, 1999.

[71] C. D. Ohl, O. Lindau, and W. Lauterborn. Luminescence from Spherically and Aspherically Collapsing Laser Induced Bubbles. Phys. Rev. Lett., 80(2):393-396, 1998.

[72] A. Pearson, J. R. Blake, and S. R. Otto. Jets in bubbles. J. Eng. Math., 48:391-412, 2004.

[73] A. Philipp, M. Delius, C. Scheffczyk, A. Vogel, and W. Lauterborn. Interaction of lithotripter-generated shock waves with air bubbles. J. Acoust. Soc. Am., 93:2496-2509, 1993.

[74] A. Philipp and W. Lauterborn. Cavitation erosion by single laserproduced bubbles. J. Fluid Mech., 361:75-116, 1998.

[75] Lord Rayleigh. On the pressure developed in a liquid during the collapse of a spherical cavity. Phil. Mag., 34:94-98, 1917.

[76] G. N. Sankin. Spherical Focusing of Acoustic Pulses in a Liquid. Acoust. Phys., 50(2):212-221, 2004.

[77] G. N. Sankin, W. N. Simmons, S. L. Zhu, and P. Zhong. Shock Wave Interaction with Laser-Generated Single Bubbles. Phys. Rev. Lett., 95:034501, 2005.

[78] G. N. Sankin and P. Zhong. Interaction between shock wave and single inertial bubbles near an elastic boundary. Phys. Rev. E, 74:046304, 2006.

[79] J. P. Sferruzza, A. Birer, and D. Cathignol. Generation of very high pressure pulses at the surface of a sandwiched piezoelectric material. Ultrasonics, 38:965-968, 2000.

[80] A. Shima, K. Takayama, Y. Tomita, and N. Miura. An experimental study on effects of a solid wall on the motion of bubbles and shock waves in bubble collapse. Acustica, 48:293-301, 1981.

[81] N. D. Shutler and R. B. Mesler. A photographic study of the dynamics and damage capabilities of bubbles collapsing near solid boundaries. Trans. ASME, J. Basic Eng., 87:511-517, 1965. 
[82] J. Staudenraus and W. Eisenmenger. Fibre-optic probe hydrophone for ultrasonic and shock-wave measurements in water. Ultrasonics, 31(4):267-272, 1993.

[83] M. Strasberg. The pulsation frequency of nonspherical gas bubbles in liquids. J. Acoust. Soc. Am., 25:536-537, 1953.

[84] H. W. Strube and W. Lauterborn. Untersuchung der Kavitationskeime an der Grenzfläche Quarzglas-Wasser nach der Zentrifugenmethode. Z. Angew. Phys., 29:349-357, 1970.

[85] Universität Stuttgart. Fiber optic probe hydrophone FOPH 300, Technical Description and Manual Instruction together with Service information.

[86] A. Tezel and S. Mitragotri. Interactions of Inertial Cavitation Bubbles with Stratum Corneum Lipid Bilayers during Low-Frequency Sonophoresis. Biophys. J., 85:3502-3512, 2003.

[87] Y. Tomita. Interaction of a Shock Wave with a Single Bubble. In: Shock Wave Science and Technology Reference Library, Volume 1, Multiphase Flows I, Ed. Marinus E. H. van Dongen, chapter 2, pages 35-66. Springer Berlin Heidelberg, 2007.

[88] Y. Tomita and T. Kodama. Interaction of laser-induced cavitation bubbles with composite surfaces. J. Appl. Phys., 94(5):2809-2816, 2003.

[89] Y. Tomita, T. Obara, K. Takayama, and M. Kuwahara. Cavitation phenomena in extracorporeal microexplosion lithotripsy. Shock Waves, 3:149-157, 1994.

[90] Y. Tomita, P. B. Robinson, R. P. Tong, and J. R. Blake. Growth and collapse of cavitation bubbles near a curved rigid boundary. J. Fluid Mech., 466:259-283, 2002.

[91] Y. Tomita and A. Shima. Mechanisms of impulsive pressure generation and damage pit formation by bubble collapse. J. Fluid Mech., 169:535564, 1986.

[92] Y. Tomita, A. Shima, and T. Ohno. Collapse of multiple gas bubbles by a shock wave and induced impulsive pressure. J. Appl. Phys., 56(1):125$131,1984$.

[93] Y. Tomita, A. Shima, and K. Takahashi. The collapse of a gas bubble attached to a solid wall by a shock wave and the induced impact pressure. J. Fluids Eng., 105:341-349, 1983. 
[94] Y. Tomita, A. Shima, and K. Takayama. Formation and limitation of damage pits caused by bubble-shock wave interaction. Proc. $1988 \mathrm{Na}$ tional Symp. on Shock Wave Phenomena (ed. K. Takayama), Institute of Fluid Science, Tohoku University, Sendai., pages 149-160, 1989.

[95] A. Vogel, S. Busch, and U. Parlitz. Shock wave emission and cavitation bubble generation by picosecond and nanosecond optical breakdown in water. J. Acoust. Soc. Am., 100(1):148-165, 1996.

[96] A. Vogel, W. Hentschel, J. Holzfuss, and W. Lauterborn. Cavitation bubble dynamics and acoustic transient generation in ocular surgery with pulsed neodymium: YAG lasers. Ophthalmology, 93:1259-69, 1986.

[97] A. Vogel and W. Lauterborn. Acoustic transient generation by laserproduced cavitation bubbles near solid boundaries. J. Acoust. Soc. Am., 84:719-731, 1988.

[98] A. Vogel, W. Lauterborn, and R. Timm. Optical and acoustic investigations of the dynamics of laser-produced cavitation bubbles near a solid boundary. J. Fluid Mech., 206:299-338, 1989.

[99] A. Vogel, K. Nahen, D. Theisen, and J. Noack. Plasma Formation in Water by Picosecond and Nanosecond Nd:YAG Laser Pulses-Part I: Optical Breakdown at Threshold and Superthreshold Irradiance. IEEE J. Sel. Top. Quantum Electron., 2(4):847-860, 1996.

[100] Z. Q. Wang, P. Lauxmann, C. Wurster, M. Köhler, B. Gompf, and W. Eisenmenger. Impluse response of a fiber optic probe hydrophone determined with shock waves in water. J. Appl. Phys., 85:2514-2516, 1999 .

[101] N. Weiss, M. Delius, S. Gambihler, P. Dirschell, A. Goetz, and W. Brendel. Influence of the shock wave application mode on the growth of A-Mel 3 and SSK2 tumors in vivo. Ultrasound Med. Biol., 16(6):595-605, 1990.

[102] B. Wolfrum. Cavitation and shock wave effects on biological systems. $\mathrm{PhD}$ thesis, Universität Göttingen, 2004.

[103] B. Wolfrum, T. Kurz, R. Mettin, and W. Lauterborn. Shock wave induced interaction of microbubbles and boundaries. Phys. Fluids, 15(10):29162922, 2003.

[104] B. Wolfrum, R. Mettin, T. Kurz, and W. Lauterborn. Observations of pressure-wave-excited contrast agent bubbles in the vicinity of cells. Appl. Phys. Lett., 81(26):5060-5062, 2002.

[105] X. Xi and P. Zhong. Improvement of stone fragmentation during shockwave lithotripsy using a combined EH/PEAA shock-wave generator-in vitro experiments. Ultrasound Med. Biol., 26(3):457-467, 2000. 
[106] F. Ronald Young. Cavitation. McGraw-Hill Book Company, London, 1989.

[107] P. Zhong and C. J. Chuong. Propagation of shock waves in elastic solids caused by cavitation microjet impact. I: Theoretical formulation. $J$. Acoust. Soc. Am., 94(1):19-28, 1993.

[108] P. Zhong, H. F. Lin, X. F. Xi, S. L. Zhu, and E. S. Bhogte. Shock waveinertial microbubble interaction: Methodology, physical characterization, and bioeffect study. J. Acoust. Soc. Am., 105:1997-2009, 1999.

[109] S. Zhu and P. Zhong. Shock wave-inertial microbubble interaction: A theoretical study based on the Gilmore formulation for bubble dynamics. J. Acoust. Soc. Am., 106(5):3024-3033, 1999. 


\section{Acknowledgment}

I want to appreciate all the people who kindly helped me during my $\mathrm{PhD}$ work:

In the first place I would like to sincerely record my gratitude to Prof. Dr. Werner Lauterborn for giving me the opportunity to work in a wonderful atmosphere in the Third Physical Institute at the University of Göttingen, as well as spending his valuable time in reviewing this thesis and giving his constructive remarks and the interesting physical discussions.

I would like to express my gratitude to Prof. Dr. Ulrich Parlitz for accepting to be the second reviewer.

I would like to gratefully acknowledge Dr. Thomas Kurz for his guidance, patience, providing very helpful physical discussions and suggestions, great support and using his precious time reviewing this thesis and giving the productive comments. With his help, I got acquainted with a variety of outstanding experimental aspects.

Many thanks go to Dr. Robert Mettin who supported and helped me in several situations during this period and also to the cooperative members of his group: Fabian Reuter, Till Nowak and Andrea Thiemann.

I am grateful to Dr. Dennis Kröninger who assisted me at the beginning of my work in the lab. I learned from him several profitable points to perform the experimental work.

Also, I want to thank Hendrik Söhnholz for his very constructive supports.

I would like to express my gratitude to Dr. Karl Lautscham and Dieter Hille and their working staff in electronic and mechanical engineering groups for their marvelous support designing and building the desired equipment.

I also like to thank Thomas Geiling and Dr. Hans Werner Strube for their delightful assists.

Many thanks to the secretary of the institute, Elke Zech for her helpful aids.

I appreciate the support of Dr. Bernhard Wolfrum for his productive comments concerning simulations.

This work is funded by Deutsche Forschungsgemeinschaft in the joint DFGCNRS research group "Micro-Macro Modeling and Simulation of Liquid-Vapor Flows", DFG project LA268-24-2.

I would like to thank my family for their great supports.

There are many others who assisted me during my time in Göttingen. I gratefully acknowledge everyone. 


\title{
Lebenslauf
}

\author{
Mohsen Alizadeh \\ geboren am 28.03.1976 in Kerman, Iran \\ Staatsangehörigkeit: iranisch
}

1994

1994-2000

2000

2000-2003

2003

2003-2004

2004-2006

seit 2006
Abitur

Studium der Physik an der Shahid Bahonar Universität zu Kerman, Iran

Bachelor in Physik

Studium der Physik an der Shahid Bahonar Universität zu Kerman, Iran

Master in Physik

Lehre an Universitäten im Iran

Forschung an den Universitäten

Tübingen und Göttingen

Wissenschaftlicher Mitarbeiter am 3. Physikalischen Institut der Universität Göttingen 